\title{
Evaluierung der Förderungsgesellschaften Austria Wirtschaftsservice GmbH (aws) und Forschungsförderungsgesellschaft mbH (FFG)
}

\author{
Abschlussbericht
}

Fraunhofer ISI: Susanne Bührer, Stephanie Daimer, Knut Koschatzky KMU Forschung Austria: Sonja Sheikh, Peter Kaufmann, Sascha Ruhland mit Unterstützung von Christina Schmedes und Hendrik Berghäuser

Karlsruhe, Wien: Dezember 2017 



\section{Inhalt}

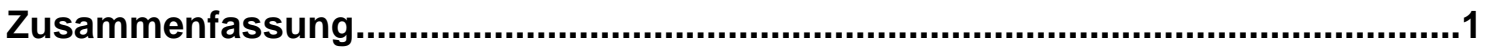

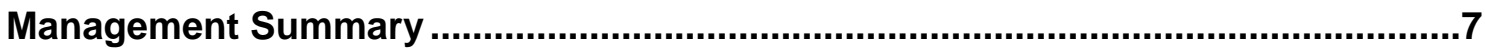

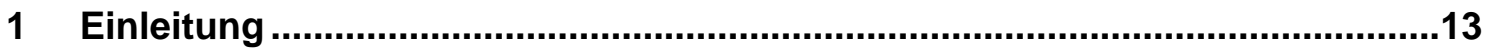

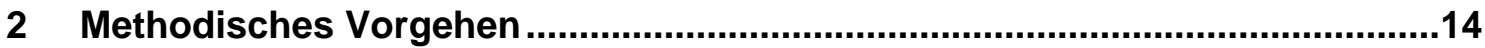

3 Evaluierung der Förderagenturen...............................................................18

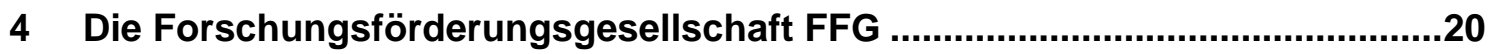

4.1 Historie und Vorläuferorganisationen..............................................21

4.2 Interne Strukturen und Prozesse ...............................................22

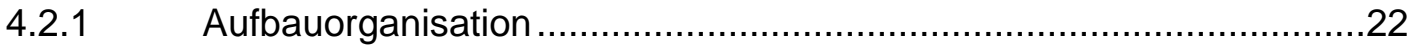

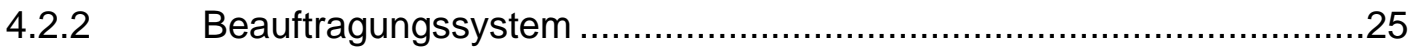

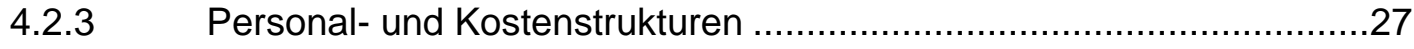

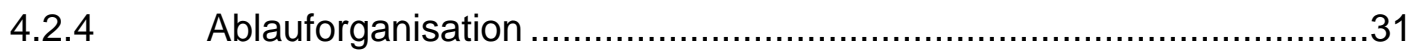

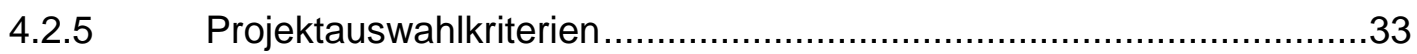

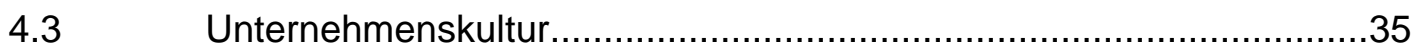

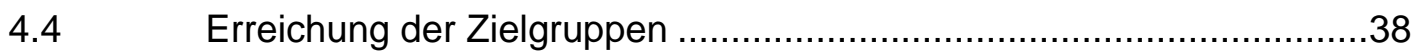

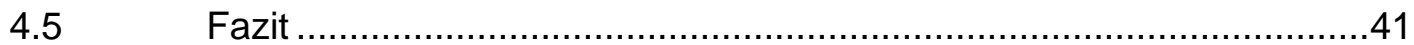

5 Die Austria Wirtschaftsservice Gesellschaft aws .......................................45

5.1 Historie und Vorläuferorganisationen............................................46

5.2 Interne Strukturen und Prozesse .................................................48

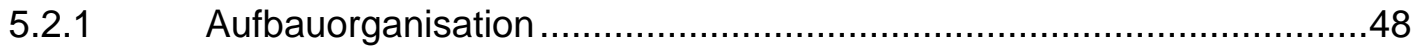




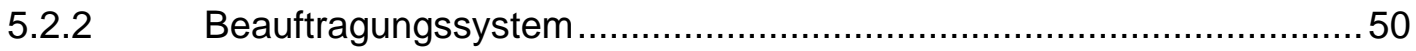

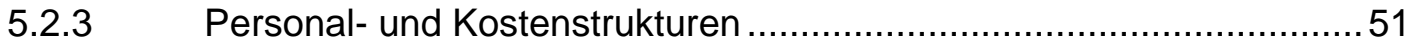

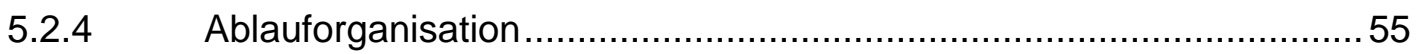

5.2.5 Management finanzieller Risiken............................................ 57

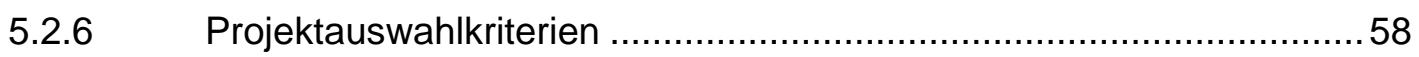

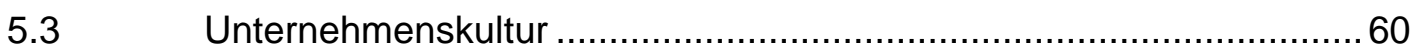

Erreichung der Zielgruppen .......................................................... 62

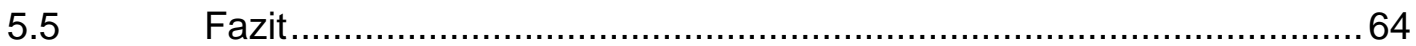

6 Positionierung und Koordination der Agenturen ........................................67

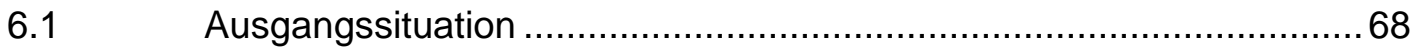

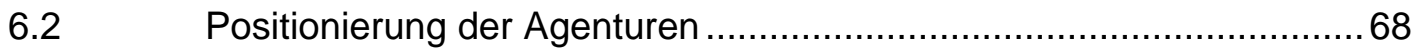

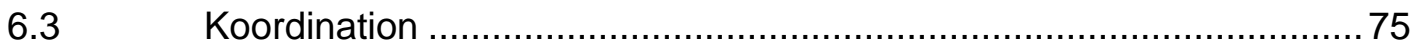

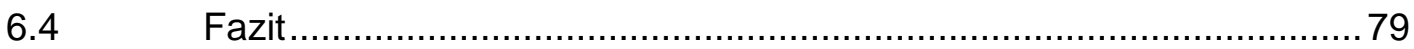

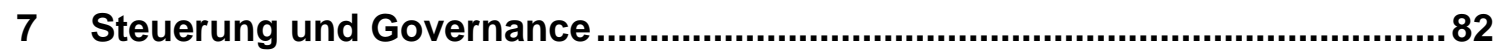

7.1 Ausgangssituation und Evaluierungsansatz ................................. 83

7.2 Zielsetzung und methodische Vorgehensweise .............................. 85

7.3 Arbeitsteilung und Rollenverständnis .......................................... 87

S.4 Steuerungsmechanismen und -kanäle ........................................... 93

Einfluss und Steuerbarkeit ....................................................... 98

7.6 Bewertung vor dem Hintergrund des Prinzipal-Agent Problems .......100

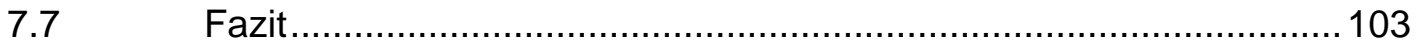

8 Internationaler Vergleich: Ausgewählte Fördergesellschaften anderer

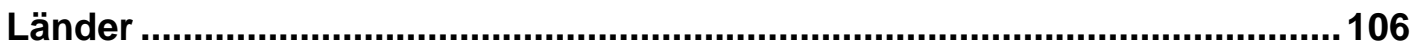

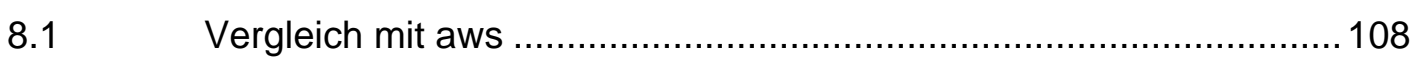




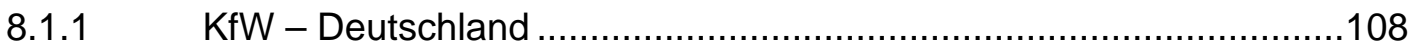

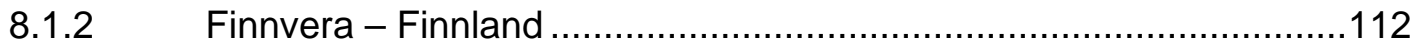

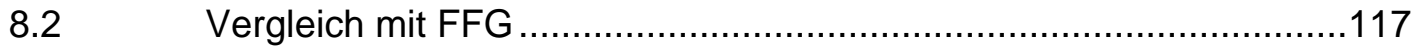

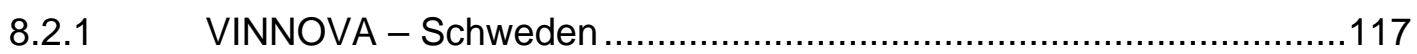

8.2.2 VLAIO - Flandern, Belgien .......................................................122

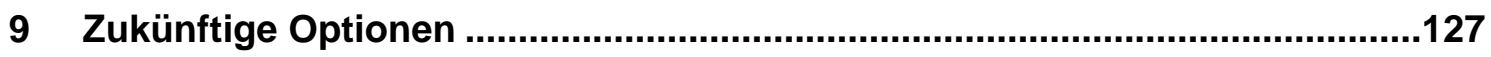

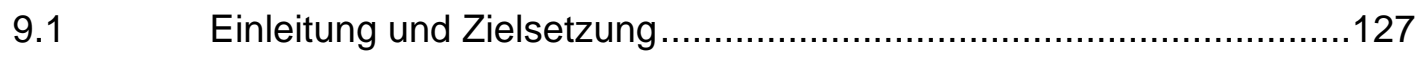

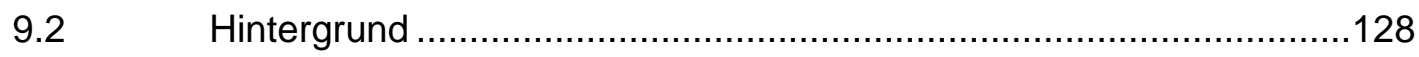

9.3 Drei Optionen für die Zukunft................................................... 130

9.3.1 Agencification Stufe 1: Weiterführung des Status quo......................130

9.3.2 Agencification Stufe 2: Weiterentwicklung des Status quo ...............132

9.3.3 Agencification Stufe 3: Höhere Autonomie durch Globalsteuerung...135

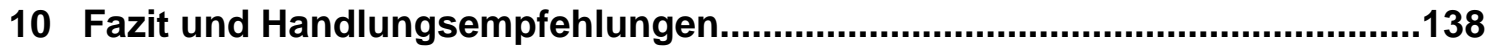

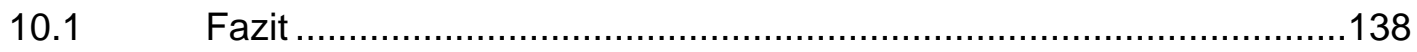

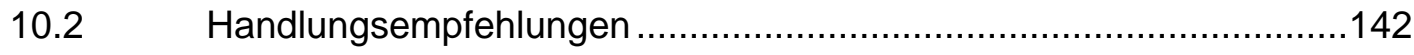

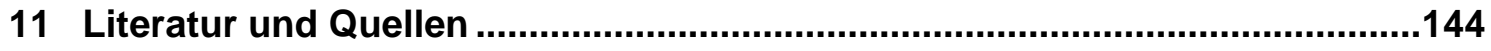

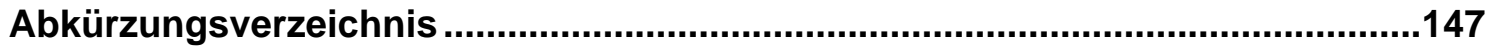




\section{Tabellen}

Tabelle 1: $\quad$ Fallzahlen und Rücklaufquoten der Online-Surveys ….................. 15

Tabelle 2: $\quad$ Übersicht über die durchgeführten Interviews .............................. 16

Tabelle 3: $\quad$ Für den internationalen Vergleich ausgewählte Organisationen .... 17

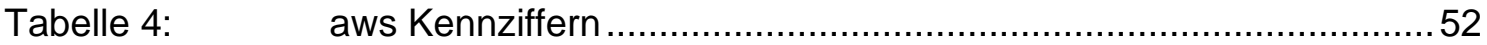

\section{Abbildungen}

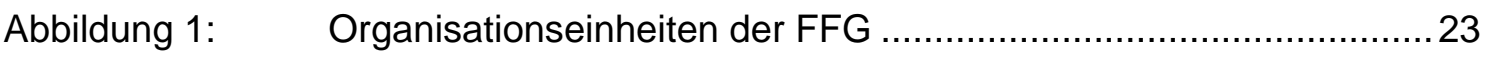

Abbildung 2: $\quad$ Entwicklung der operativen Mittel und Personalausstattung ..........28

Abbildung 3: $\quad$ Entwicklung der Vollzeitäquivalente nach Bereichen .....................29

Abbildung 4: $\quad$ Bewertung der internen Strukturen und Prozesse der FFG ............ 31

Abbildung 5: $\quad$ Zufriedenheit mit dem Arbeitsumfeld ............................................. 32

Abbildung 6: $\quad$ Förderkriterien aus Sicht der FFG-MitarbeiterInnen .......................33

Abbildung 7: $\quad$ Förderkriterien aus Sicht der Ministerien.................................... 34

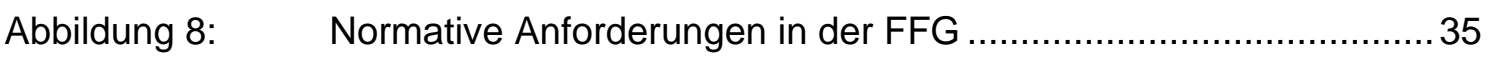

Abbildung 9: $\quad$ Unternehmenskultur aus Sicht der FFG-Mitarbeiterlnnen ...............37

Abbildung 10: Veränderung der Zielgruppenerreichung seit der Fusion zur FFG. 38

Abbildung 11: $\quad$ Beurteilung der Veränderung durch die FFG-Fusion .................... 39

Abbildung 12: $\quad$ Einschätzung der FFG hinsichtlich ausgewählter Qualitätsmerkmale.

Abbildung 13: $\quad$ Wie einfach oder kompliziert ist es aus Ihrer Sicht, die für Ihr Anliegen am besten geeignete Unterstützungsmaßnahme bei der FFG zu finden?............................................................... 41

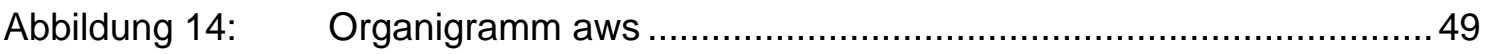

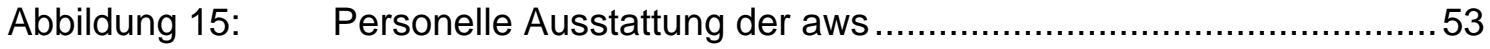

Abbildung 16: Zufriedenheit der MitarbeiterInnen in der aws ..............................55

Abbildung 17: $\quad$ Normative Anforderungen und Wertschätzung in der aws .............57

Abbildung 18: Förderkriterien aus Sicht der aws-MitarbeiterInnen.......................59

Abbildung 19: $\quad$ Unternehmenskultur aus Sicht der aws-Mitarbeiterlnnen...............60

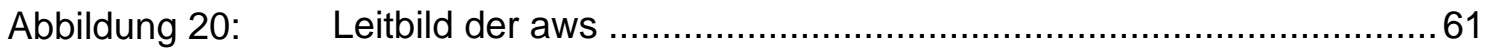


Abbildung 21: Veränderung der Zielgruppenerreichung seit der Fusion zur aws ..62

Abbildung 22: $\quad$ Einschätzung der aws hinsichtlich ausgewählter Qualitätsmerkmale.

Abbildung 23: Beurteilung der Veränderung durch die aws-Fusion.

Abbildung 24: Wie einfach oder kompliziert ist es aus Ihrer Sicht, die für Ihr Anliegen am besten geeignete Unterstützungsmaßnahme bei der aws zu finden?....

Abbildung 25: Verbindung der aws-Schwerpunkte mit den Wirkungszielen 69

Abbildung 26: Die Wirkungsfelder der FFG gemäß FTI und FFG Richtlinien........70

Abbildung 27: Wie klar ist Ihnen, an welche Agentur Sie sich bei den exemplarisch aufgelisteten Anliegen wenden sollen? .74

Abbildung 28: Wie klar ist Ihnen, an welche Agentur Sie sich bei den exemplarisch aufgelisteten Anliegen wenden sollen? .75

Abbildung 29: Steuerungskanäle der Ressorts vis-à-vis den Agenturen. .94

Abbildung 30: Operationale Autonomie der KfW 110

Abbildung 31: $\quad$ Finanzielle Autonomie der KfW

Abbildung 32: Personelle Autonomie der KfW

Abbildung 33: Operationale Autonomie der Finnvera.

Abbildung 34:

Finanzielle Autonomie der Finnvera

Abbildung 35:

Personelle Autonomie der Finnvera

Abbildung 36:

Operationale Autonomie der VINNOVA.

Abbildung 37: Finanzielle Autonomie der VINNOVA ....................................120

Abbildung 38: $\quad$ Personelle Autonomie der VINNOVA …....................................121

Abbildung 39: $\quad$ Operationale Autonomie der VLAIO ….......................................124

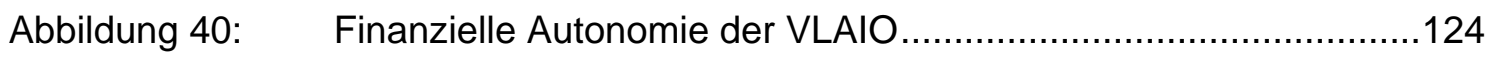

Abbildung 41: $\quad$ Personelle Autonomie der VLAIO .................................................125 



\section{Zusammenfassung}

Anlass und Ziele der Evaluierung

Im Juni 2016 wurden das Fraunhofer ISI und die KMU Forschung Austria mit der Evaluierung der Förderungsgesellschaften FFG und aws beauftragt. Ziel der Evaluierung war es, über 10 Jahre nach Umsetzung der Strukturreformen Bilanz über deren Zielerreichung zu ziehen. Die Evaluierung basierte auf einem Methodenmix aus qualitativen und quantitativen Vorgehensweisen, der neben einer umfangreichen Dokumenten- und Datenanalyse mehrere standardisierte Befragungen (in den Ressorts, den Agenturen sowie bei den KundInnen der Agenturen) sowie insgesamt über 80 Interviews und fünf Workshops umfasste. Die Evaluierung wurde im Juni 2017 abgeschlossen.

Laut Leistungsbild sollte der Ausgangspunkt der Evaluierung die Annahmen und Ziele sein, die zu den Strukturreformen im Förderungsbereich und zur Gründung der FFG und aws führten. Diese Ziele und Annahmen waren (ibd.):

- Reduktion organisatorischer und inhaltlicher Komplexität,

- Lösung immanenter Koordinationsprobleme durch die Schaffung von ressortübergreifenden Förderungsgesellschaften,

- Erhöhung der politischen Steuerungsfähigkeit gegenüber den Zielgruppen,

- Verbesserung der Umsetzungschancen von Regierungszielen, nämlich Effizienz und Intensivierung des Ressourceneinsatzes.

Diese vier Ziele wurden für die empirische Umsetzung der Evaluierung in vier Leitthemen zusammengefasst und untersucht, (1) wie die interne Struktur und Organisation der Agenturen charakterisiert werden kann, (2) welche Unternehmenskulturen sich in den Agenturen herausgebildet haben, (3) wie sich die Agenturen innerhalb des österreichischen Innovationssystems positionieren und (4) wie die Agenturen von den Eigentümerressorts gesteuert werden. Zusätzlich hat das Evaluationsteam drei Zukunftsoptionen für die zukünftige Ausgestaltung der Beziehungen zwischen Eigentümerressorts und Agenturen entwickelt.

Das zentrale Ergebnis dieser Studie lässt sich wie folgt zusammenfassen: Die Ziele der Strukturreformen wurden teilweise erreicht. Mit der FFG und der aws verfügt Österreich über zwei hochprofessionell agierende Förderungsagenturen. Die Agenturen können jedoch ihre volle Leistungsfähigkeit aufgrund der gesetzten Rahmenbedingungen nicht entfalten. Im Folgenden fassen wir zusammen, auf welche Erkenntnisse wir diese Kernaussagen stützen. 


\section{Organisationsanalyse}

Beide Agenturen agieren nach einem erfolgreich vollzogenen Fusionsprozess hochprofessionell und effizient. Im Zuge der Fusion und der dadurch entstehenden relativ großen Organisationseinheiten wurden zur Gewährleistung einer hohen Professionalität, Serviceorientierung, Effektivität und Effizienz zahlreiche Prozesse und Strukturen entwickelt und implementiert, die sich im Großen und Ganzen bewährt haben. Beide Agenturen haben erfolgreich Qualitätskontrollen aufgebaut, Systeme zur Mitarbeiterqualifizierung etabliert und eine hohe Praxisnähe aufgebaut. Die entwickelten Werkzeuge erhöhen die Effizienz des Agenturhandelns, aber sie schaffen gleichzeitig auch Inflexibilität.

Innerhalb der Organisation gibt es einzelne kritische Stimmen, die - in beiden Agenturen - vor allem Fragen der Aufstiegskriterien und des Einbezugs in Entscheidungsprozesse betreffen. Ein Preis für die hohe Prozessorientierung ist außerdem ein gewisser Mangel an Flexibilität bei der Abwicklung von Förderprogrammen. Angesichts der großen Bedeutung administrativer und rechtlicher Vorgaben aufgrund beispielsweise des Beihilferechts sowie der auch in Österreich zu beobachtenden starken Rolle des Rechnungshofes ist eine hohe Prozesstreue jedoch unverzichtbar, um Entscheidungen abzusichern.

Beide Agenturen, und vor allem ihre Untereinheiten bzw. Bereiche, sind durch komplexe Aufgabenstellungen geprägt, so dass sie zwar einerseits eine hohe gemeinsame Identität aufweisen, andererseits aber im Alltagshandeln durchaus unterschiedliche Orientierungen zu finden sind, die auf die große Vielfalt unterschiedlicher Beauftragungssysteme zurückzuführen sind. In Teilen spielen auch noch die Sozialisierungen in einer der Vorgängerorganisationen eine Rolle.

Eine Herausforderung für beide Agenturen ist eine teils kritische Einschätzung ihrer KundInnen zu mehreren Qualitätsmerkmalen: Zwar wird der Erfolg des Fusionsprozesses einhellig positiv gewürdigt, vor allem hinsichtlich allgemeiner Hilfestellung und Kommunikation, Unterstützung der jeweiligen Anliegen und der Klarheit von Zuständigkeiten; bemängelt wird von den KundInnen hingegen Transparenz, Geschwindigkeit und Flexibilität der Entscheidungen. Diese Herausforderung anzugehen ist Aufgabe aller Beteiligten, d.h. sie wird ohne eine aktive und verbesserte Rahmensetzung durch die Eigentümerressorts nicht zu bewerkstelligen sein.

\section{Positionierung und Koordination}

Die beiden Agenturen sind mit ihren spezifischen Geschäftsmodellen im nationalen innovationspolitischen Umfeld klar positioniert. Sie fungieren in weiten Teilen als one-stopshop für Forschung, Technologieentwicklung und Innovation (FFG) sowie investive Un- 
ternehmensförderung und Innovationsunterstützung (aws). Eine stärkere horizontale Koordination zwischen den Agenturen im österreichischen Fördersystem wurde durch die Strukturreformen 2002-2004 erreicht. Dies trifft jedenfalls auf die nun in Form von Bereichen weitergeführten vormaligen Agenturen zu, die aufgrund der Zusammenführung vielfältige Vorteile ausschöpfen konnten.

Es gibt jedoch noch Optimierungspotenzial. Zum Beispiel können agenturinterne Prozesse nicht gänzlich standardisiert werden, wenn die Auftraggeber unterschiedliche Bedürfnisse für Dokumentation und Reporting aufweisen (z.B. Bereich Energie/Klimafonds), oder unterschiedliche Governance-Strukturen der Eigentümer die Möglichkeiten zur Harmonisierung von Organisationsstrukturen hintanhalten (z.B. EIP/TP in der FFG). Dieses Optimierungspotenzial kann nur unter veränderten Rahmenbedingungen ausgeschöpft werden, also indem die Agenturen ihre Förderinstrumente stärker selbst definieren können und es so zu einer strategischen Themensteuerung (durch die Auftraggeber) kommen kann.

Die Zusammenarbeit und Koordination zwischen den beiden Agenturen kann, auch wenn die Schnittmenge quantitativ relativ klein ist, insofern noch geschärft werden, indem die Kriterien zur Beauftragung der einzelnen Agenturen noch expliziter gemacht werden. Damit wird man Überlappungsbereiche klein halten, aber nicht völlig beseitigen können. Da eine trennscharfe Abgrenzung nicht gänzlich möglich ist, muss eine gute Zusammenarbeit und Abstimmung an diesen Randbereichen erfolgen, die bereits im Stadium des Programmdesigns beginnt, indem die Auftraggeber mit den Agenturen eine gemeinsame Lösung suchen.

\section{Steuerung und Governance}

Ein zentraler Befund der vorliegenden Evaluierung hinsichtlich der Steuerung lautet, dass das gesamte Governancesystem von (anhaltend) hoher Komplexität geprägt ist. Dies ist einerseits darin begründet, dass die Steuerung der Agenturen nach wie vor über zahlreiche gleichzeitig zu bedienende Ausführungsverträge erfolgt und andererseits im Fehlen eines einheitlichen, koordinierten Steuerungsverständnisses seitens der Eigentümer. Auch daher lassen sich abweichende Selbst- und Fremdbilder bezüglich der Arbeitsteilung zwischen Ressorts und Agenturen feststellen, die insbesondere auf die unvollständige Delegation von Umsetzungsaufgaben an die Agenturen verweisen. Die Steuerung der Agenturen geht von unterschiedlichen Akteuren aus (den beiden Eigentümerressorts sowie anderen Auftraggebern, den jeweiligen Fachabteilungen und Kabinetten). Die verschiedenen Akteure nutzen unterschiedliche Steuerungsmechanismen und -kanäle und stimmen sich in der Regel nicht ausreichend ab, was teilweise sogar 
widersprüchliche Steuerungsimpulse für die Agenturen bedeutet. Die Situation wird insbesondere durch die steigende Bedeutung der Kabinette und dem direkten Zugang der Agenturen hierhin zunehmend komplexer. Damit entsteht eine Situation, in der nicht länger eindeutig klar ist, wer letztlich die Rolle des Prinzipals einnimmt und dementsprechend die Vorgaben für die Agenten/Agenturen macht. Anstatt also Unsicherheiten für alle Beteiligten zu minimieren, entstehen eher zusätzliche. Insgesamt entsteht der Eindruck, dass eine Steuerung der Agenturen als Ganzes auch aufgrund der in den Rahmenbedingungen angelegten relativen Steuerungsschwäche der Ressorts derzeit kaum möglich ist. Es scheint ein Steuerungsvakuum zu geben, das am ehesten noch die Agenturen für sich zu nutzen wissen. Auch der vielfach geäußerte Vorwurf des Mikromanagements durch die Ressorts erklärt sich nicht zuletzt als Reaktion auf die als zu schwach wahrgenommene (eigene) Steuerungskapazität.

Während die Agenturen auch bei weitgehend unveränderten Rahmenbedingungen aufgrund etwa der Kumulation strategischer Intelligenz und des Zuwachses an Einfluss stärker bzw. autonomer geworden sind, hat es weder eine adäquate Neujustierung des Systems (auch nicht infolge der Systemevaluierung) gegeben noch eine umfassende analoge Ko-Evolution innerhalb der Ressorts. Letzteres betrifft vor allem die ausgebliebene Entwicklung eines neuen Steuerungsverständnisses und entsprechender Einstellungen sowie Fähigkeiten vor dem Hintergrund zunehmend professionalisierter Umsetzungsprozesse in den Agenturen.

Die Lösung liegt in einer stärkeren politisch-strategischen Führung durch die Prinzipale mit klaren Definitionen von Zielen, Inhalten, Rollen und Schnittstellen zwischen Eigentümern/Auftraggebern und Agenturen sowie darüber hinaus darin, den Agenturen über die vollständige Delegation der Umsetzung von Fördermaßnahmen operativ mehr Freiheitsgrade einzuräumen, damit sie mit ihrer neu gewonnenen Flexibilität ihre Positionierung im österreichischen Innovationssystem noch weiter schärfen, und mit verschlankten Produkten und Prozessen ihre KundInnen noch besser adressieren und die Wirkung der von ihnen angebotenen Maßnahmen optimieren können.

\section{Erreichung der Strukturreformziele}

Zusammenfassend können wir festhalten, dass durch den Zusammenschluss der Agenturen und die dort etablierten einheitlichen Strukturen und Prozesse eine Reduktion der organisatorischen Komplexität sowie eine Reduzierung von Koordinationsproblemen erfolgte; die inhaltliche Komplexität hat sich jedoch über die Jahre aufgrund des förderpolitischen Umfeldes tendenziell eher erhöht, dies hat jedoch wenig mit den Strukturreformen und der Entwicklung der Agenturen zu tun. Die politische Steuerungsfähigkeit ge- 
genüber den Zielgruppen hat sich im Vergleich zu einem Szenario mit den acht Vorgängerorganisationen sicherlich erhöht. Die Agenturen weisen inzwischen einen hohen Professionalisierungsgrad auf, der mittelbar auch eine Verbesserung der Umsetzungschancen von Regierungszielen bedeutet.

Es gibt jedoch nach wie vor Herausforderungen, die aus Sicht des Evaluatorenteams unter anderem adressiert werden können, wenn am Verhältnis zwischen Eigentümern und Agenturen einige (zentrale) Änderungen vorgenommen werden.

\section{Fazit und Ausblick}

Österreich gibt im internationalen Vergleich viel Geld für angewandte Forschung (inkl. der Forschungsprämie) und die Wirtschaftsförderung aus. Um die mit einer so großen Fördersumme möglichen Impulse wirkungsreich zu setzen, anstatt vorwiegend strukturkonservierend zu wirken, bedarf es einer einheitlichen strategischen Planung und Verantwortung. Dies ist umso wichtiger, als Forschung, Innovation und Investitionen in Unternehmen - und damit auch deren Förderung - von einer hohen Veränderungsdynamik und gesellschaftlichen Herausforderungen (u.a. die Digitalisierung) getrieben sind, die rasches und strategisches Agieren erfordert.

Die Agenturen müssen in die Lage versetzt werden, dass sie dynamisch, risikobereit und verantwortlich Förderportfolios gestalten können, die politisch gesetzte Prioritäten adressieren und umsetzen. Die entsprechenden Rahmenbedingungen müssen gleichzeitig die Steuerungsfähigkeit der Eigentümerressorts stärken (nicht zuletzt, um der Problematik ansonsten fehlender mittelbarer demokratischer Kontrolle und Legitimierung zu begegnen und die Agenturen als Instrument der Umsetzung politischer Willensbildung zu erhalten) und das Governancesystem auf eine vertrauensbasierte, ergebnisorientierte Grundlage stellen.

Als Fazit legen wir den Eigentümern daher nahe, gemäß dem Leitsatz "Leistungsfähigkeit durch Eigenverantwortung" vor allem die finanzielle und operationale Autonomie der Agenturen zu stärken. Die Evaluierung gibt anhand von drei Zukunftsoptionen Anregungen, wie ein solcher Prozess gestaltet werden könnte und wie sich ein verändertes Verhältnis zwischen Agenturen und Eigentümern auf die vier als relevant herausgearbeiteten Dimensionen (1) Governance, (2) Finanzierung, (3) Aufgaben, und (4) Organisationsstrukturen und Managementprozesse auswirken können. Hierzu haben wir drei denkbare Ausprägungen von Agencification differenziert:

- Agencification Stufe 1: Weiterführung des Status quo,

- Agencification Stufe 2: Weiterentwicklung des Status quo,

- Agencification Stufe 3: Höhere Autonomie durch Globalsteuerung. 
Die Fortführung des Status quo führt zu keinen Veränderungen und dementsprechend alle Stärken und Schwächen des derzeitigen Systems weiter. Obgleich die Weiterschreibung der aktuellen Situation eine Reihe von Nachteilen beinhaltet, nämlich die konsequente Perpetuierung der im vorliegenden Bericht herausgearbeiteten Probleme, gibt es auch einen Vorteil: alle Akteure haben für das geltende System entsprechende Routinen entwickelt.

Zukunftsoption 2 "Weiterentwicklung des Status quo" fokussiert auf eine Korrektur insbesondere der auf die Governance bezogenen Aspekte in Richtung Stärkung der Ressorts durch eine konsequentere strategische Steuerung der Agenturen, sowie gleichzeitige Stärkung der Agenturen durch eine größere Unabhängigkeit auf der operativen Ebene. Die Agenturaufgaben wären mit Ausnahme der Aufwertung des Instrumentendesigns und größeren Freiheitsgraden bei der Programmentwicklung damit weitgehend wie bisher gestaltet; der Prinzipal würde seine Kontrollaufgaben jedoch stärker wahrnehmen.

Zukunftsoption 3 manifestiert den Übergang von der Detailsteuerung hin zu einer ergebnisorientierten Steuerung "auf Abstand", die mittels eines Globalbudgets zwar die gewünschten Wirkungen der Agentur mittels Zielformulierung und der Definition von Wirkungsindikatoren vorgibt, die Verteilung des Budgets auf Instrumente jedoch großteils der Agentur überlässt. Damit erfolgt eine Übertragung von mehr Entscheidungskompetenz und Verantwortung an die Agentur, während sich der Prinzipal aufgrund der entstehenden Freiräume verstärkt auf die politisch-strategischen Aspekte, die Gestaltung von strukturierten Lernprozessen zur Minimierung von Informationsasymmetrien (durch Transparenz und Partizipation zur Unterstützung des Agenda-Settings), sowie die Controllingfunktion fokussiert.

Der zentrale Vorteil dieser Zukunftsoption liegt darin, dass eine höhere Flexibilität der Umsetzung in der Agentur zu klarer kommunizierbaren Produkten, eine Beschleunigung von Prozessen zu einer besseren Zielgruppenansprache, und kurze Lernschleifen sowie eine flexible Kombination von Instrumenten zu einer größeren Hebelwirkung der eingesetzten Fördermittel führen.

\section{Danksagung}

Unser ganz besonderer Dank gilt dem externen ExpertInnenkreis, der uns während der Studie kontinuierlich beratend unterstützt und damit einen unverzichtbaren Beitrag zum Gelingen der Studie geleistet hat. Diese ExpertInnen waren (in alphabetischer Reihenfolge): Prof. Dr. Markus Höllerer (Wirtschaftsuniversität Wien), Dr. Wilhelm Krull (VW Stiftung), Dr. Vivien Lo (Kreditanstalt für Wiederaufbau, KfW), Dr. Jürgen Marchart (AVCO), Dr. Michael Stampfer (WWTF). 


\section{Management Summary}

\section{Background and objectives of the evaluation}

The Fraunhofer ISI and KMU Forschung Austria (Austrian Institute for SME Research) were charged with evaluating the funding agencies aws (Austria Wirtschaftsservice - the Austrian Federal Promotional Bank) and the Austrian Research Promotion Agency (FFG) in June 2016. The objective was to weigh up whether the goals had been achieved in the 10 years since structural reforms were implemented. The evaluation was based on a mix of qualitative and quantitative methods that included several standardized questionnaires (in the ministries, the agencies and among the agencies' clients) alongside extensive document and data analyses and a total of more than 80 interviews and five workshops. The evaluation was completed in June 2017.

According to the scope of the work, the evaluation should start with the assumptions and objectives that led to the structural reforms in the funding sector and the foundation of the FFG and aws. These goals and assumptions were:

- Reduce the complexity of organization and contents,

- Solve intrinsic coordination problems by creating cross-ministerial funding agencies,

- Increase the ability to steer policies towards target groups,

- Improve the chances of the government's objectives being implemented, namely efficiency and intensifying the use of resources.

These four goals were grouped into the following four key topics for the empirical evaluation and then analysed: (1) How the internal structure and organization of the agencies can be characterized; (2) which corporate cultures have evolved in the agencies; (3) the agencies' position within the Austrian innovation system; and (4) how the agencies are governed by the owners, i.e. the ministries. In addition, the evaluation team developed three future options of how to structure relations between owners and agencies in the future.

The study's main result can be summed up as follows: The structural reform's objectives have been partially achieved. With the FFG and the aws, Austria now has two highly professional funding agencies at its disposal. However, the agencies are not able to develop their full productive capacity due to the framework conditions in place. In the following, we summarize the findings underpinning these core statements. 


\section{Organization analysis}

Both agencies are functioning efficiently and highly professionally following a successfully completed fusion process. Numerous processes and structures were developed and implemented in the course of this fusion to guarantee a high level of professionalism, service orientation, effectiveness and efficiency. The relatively large organizational units this resulted in have proven generally effective. Both agencies have been successful in constructing quality controls, systems of staff qualification and a high level of practical relevance. The developed tools have the advantage of increasing the efficiency of the agencies' activities but create inflexibility at the same time.

As in every organization, criticisms are voiced by a few individuals that - again in both agencies - mainly concern the criteria for promotion and inclusion in decision processes. In addition, the price for the strong process orientation is a certain lack of flexibility when managing programs. In view of the importance of administrative and legal stipulations, for example based on the law governing state aid, and the strong role of the national audit office that can also be observed in Austria, a high level of adherence to processes or "sticking to the rules" is essential to secure decisions.

On the one hand, both agencies, and above all their sub-units or sections, are characterized by complex tasks so that they share a common identity. On the other hand, different orientations are found in daily routines that can be traced back to the very wide variety of different commissioning systems. In some parts, however, the socializations in one of the predecessor organizations still play a role.

One challenge for both agencies is their clients' partly critical assessment of several quality characteristics: True, there is praise from all sides for the success of the fusion process, especially with regard to general aid and communication, support for the respective issue and clarity of responsibilities; but clients also have criticisms about the transparency, speed and flexibility of decisions. Tackling this challenge is a task for all those involved, i.e. it cannot be managed without the owners setting an active and improved regulatory framework.

\section{Positioning and coordination}

With their specific business models, both agencies hold clear positions in the national innovation policy environment. To a large extent, they function as a one-stop-shop for research, technology development and innovation (FFG) as well as for business funding and innovation support (aws). Stronger horizontal coordination between the agencies in the Austrian funding system was achieved by the structural reforms 2002-2004. This certainly applies to the eight former agencies, which are now organised as sections in 
the aws and FFG. They have been able to exploit a wide range of advantages due to the mergers.

However, there is still potential for optimization. For example, agency-internal processes cannot be completely standardized if clients have varying requirements for documentation and reporting, or if the owners' varying governance structures stand in the way of harmonizing organizational structures. This optimization potential can only be exploited under altered framework conditions, for instance by enabling the agencies to define funding instruments themselves to a greater extent and thus permitting the strategic management of topics (by the ministries).

Even though the overlap between the two agencies is relatively small, cooperation and coordination between can be enhanced by making the criteria for commissioning the individual agencies even more explicit. This would keep overlapping areas small, but it is not possible to eliminate them altogether. Since it is not completely possible to separate the two agencies along strictly defined lines, good cooperation and coordination is required for these peripheral areas. This should already begin at the stage of designing programs when contracting authorities search for a joint solution together with the agencies.

\section{Steering and governance}

One key finding of this evaluation concerning steering the agencies is that the entire governance system is characterized by (continued) high complexity. On the one hand, this is explained by the fact that the agencies are still governed by a large number of implementation agreements and, on the other hand, a consistent and coordinated understanding of how the steering processes should be defined and executed is missing on the part of the owners. This is also why deviating self-images and external images can be found regarding the division of labour between ministries and agencies, which refers to the incomplete delegation of implementation tasks to the agencies. The agencies are governed by different actors (owners and other clients, the respective specialist departments and cabinets). The various actors use different steering mechanisms and channels and usually do not consult and coordinate with each other enough, which leads to partially contradictory steering impulses for the agencies. The situation is becoming ever more complex due to the growing importance of the cabinets and the agencies' direct access to them. This results in a situation where it is no longer completely clear who plays the role of principal and accordingly makes the rules for the agents/agencies. Instead of minimizing uncertainty for all those involved, additional uncertainties are caused. In general, the impression is that, due to the ministries' relative weakness, it is hardly possible at present to steer the agencies as a whole. There seems to be a steering 
vacuum that the agencies best know how to exploit for themselves. The often expressed complaint against the ministries' micromanagement can also be explained as a reaction to the perceived weakness of their (own) steering capacity.

While the agencies have become more autonomous even under largely unaltered framework conditions due to the accumulation of strategic intelligence and increased influence, there has neither been an adequate readjustment of the system (not even as a consequence of the system's evaluation) nor a comprehensive analogue co-evolution within the ministries. The latter concerns mainly the failure to develop a new understanding of steering and the relevant new personnel and abilities against the backdrop of increasingly professionalized implementation processes in the agencies.

The solution lies in stronger policy-strategy guidance by the principals with clearly defined objectives, content, roles and intersections between owners/clients and agencies. Furthermore, agencies should be given a greater degree of freedom by completely delegating the implementation process to them so that they can further refine their position in the Austrian innovation system with their newly gained flexibility. They would then be able to target clients better with streamlined products and processes and optimize the effect of the measures offered.

\section{Achievement of the structural reform objectives}

In summary, we conclude that there has been a reduction in both organizational complexity and coordination problems due to the merger of the agencies and the structures and processes established. The complexity of the contents involved, on the other hand, has tended to increase over the years due to the funding policy environment, but this has less to do with the structural reforms and the development of the agencies. The ability to steer policy towards target groups has definitely increased compared to the scenario with the eight predecessor organizations. The agencies now show a high degree of professionalism that also directly implies better chances of implementing governmental objectives.

However, there are still challenges remaining that the evaluators believe can be addressed by making several (major) changes to the relationship between owners and agencies.

\section{Summary and outlook}

In an international comparison, Austria spends a lot on applied research (including the research premiums) and economic support (business development). Uniform strategy planning and responsibility are needed in order to set the effective impulses that are possible with such a large amount of funding instead of mainly conserving structures. 
This is even more important since research, innovation and investments in companies and therefore also their support - are driven by high dynamics and societal challenges (including digitalization) which require rapid and strategic action.

The agencies have to be in the position to design funding portfolios dynamically, responsibly and with the readiness to take risks that address and implement politically defined priorities. The relevant framework conditions have to strengthen the ministries' capacity for control (not least to address the problem of the otherwise lack of direct democratic control and legitimacy and to maintain the agencies as an instrument for implementing political decision-making) and simultaneously place the governance system on a foundation that is result-oriented and based on trust.

To sum up, we recommend to the principals that the financial and operating autonomy of the agencies should be strengthened in line with the guiding principle "Performance through individual responsibility". The evaluation makes suggestions based on three future options for how such a process could be structured and how the changed relationship between agencies and owners could impact four dimensions that were considered relevant (1) Governance, (2) Financing, (3) Tasks and (4) Organizational structures and management processes. To do so, we differentiated three conceivable versions of agencification:

- Agencification Stage 1: Continuation of the status quo,

- Agencification Stage 2: Further development of the status quo,

- Agencification Stage 3: Greater autonomy due to overall control ('Globalsteuerung').

The continuation of the status quo results in no changes and accordingly retains all the strengths and weaknesses of the current system. Although continuing the current situation includes drawbacks, namely the perpetuation of the problems presented in this report, there is also one advantage: All the actors involved have developed the corresponding routines for the system as it is at present.

Future option 2 "Further development of the status quo" focuses on correcting aspects, especially those related to governance in terms of strengthening the ministries by more consistent strategic control of the agencies and simultaneously strengthening the agencies by granting greater independence at the operating level. The agencies' tasks would be largely structured as before with the exception of improved instrument design and a greater freedom of scope when developing programs; but the principals would be more aware of their control duties.

Future option 3 manifests the transition from micro-management to result-oriented control "at a distance". The agency's desired impacts are specified using a global budget, 
formulation of objectives and definition of impact indicators, but how the budget is allocated to instruments is left to the agency itself to a large extent. This transfers more decision-making competence and responsibility to the agency while freeing up the principal to focus more on policy-strategy aspects, the design of structured learning processes to minimize information asymmetries (through transparency and participation to support agenda setting) and control.

The major advantage of this option is that greater flexibility of implementation in the agency results in products that are easier to communicate, the acceleration of processes leads to an improved target group approach and short learning loops and the flexible combination of instruments results in greater leverage of the funds used.

\section{Acknowledgment}

Our special thanks go to the external circle of experts, who supported us continuously during the study and thus made an indispensable contribution to the success of the study. These experts were (in alphabetical order): Prof. Dr. Markus Höllerer (Vienna University of Economics and Business), Dr. Wilhelm Krull (VW Foundation), Dr. Vivien Lo (Kreditanstalt für Wiederaufbau, KfW), Dr. Jürgen Marchart (AVCO), Dr. Michael Stampfer (WWTF). 


\section{$1 \quad$ Einleitung}

Im Juni 2016 wurden das Fraunhofer ISI und die KMU Forschung Austria mit der Evaluierung der Förderungsgesellschaften Austria Wirtschaftsservice $\mathrm{GmbH}$ (aws) und Forschungsförderungsgesellschaft mbH (FFG) beauftragt. Die Evaluierung wurde zwischen Juni 2016 und Juni 2017 durchgeführt.

Die FFG ist 2004 aus der Zusammenlegung von vier bis dahin bestehenden Organisationen hervorgegangen (dem Forschungsförderungsfonds für die gewerbliche Wirtschaft - FFF, der Technologieimpulse Gesellschaft zur Planung von Technologiezentren GmbH - TIG, der Österreichischen Gesellschaft für Weltraumfragen - ASA, sowie des Büros für internationale Forschungs- und Technologiekooperation - BIT), die alle der Förderung der angewandten Forschung zugeordnet waren. Die aws ist entstanden durch die Verschmelzung der Finanzierungsgarantie-Gesellschaft FGG, der Innovationsagentur und der BÜRGES Förderbank. Seit ihrer Gründung übernimmt die aws auch die Geschäfte des ERP-Fonds sowie die Geschäftsführung der Nationalstiftung.

In der Evaluierung der Förderungsgesellschaften Forschungsförderungsgesellschaft $\mathrm{mbH}$ (FFG) und Austria Wirtschaftsservice $\mathrm{GmbH}$ (aws) geht es im Kern um die Fragestellung, ob und in welchem Umfang die Ziele und Erwartungen der vor mehr als 10 Jahren erfolgten Zusammenführungen der Förderungsorganisationen erreicht wurden. Die Strukturreformziele bestanden in der

- Reduktion organisatorischer und inhaltlicher Komplexität,

- Lösung immanenter Koordinationsprobleme,

- Erhöhung der politischen Steuerungsfähigkeit gegenüber den Zielgruppen,

- Verbesserung der Umsetzungschancen von Regierungszielen.

Diese vier Gegenstandsbereiche haben wir für die empirische Umsetzung der Evaluierung in vier Leitthemen zusammengefasst und untersucht, (1) wie die interne Struktur und Organisation der Agenturen beschrieben werden kann, (2) welche Unternehmenskulturen sich in den Agenturen herausgebildet haben (Kapitel 3-5), (3) wie sich die Agenturen innerhalb des österreichischen Innovationssystems positionieren (Kapitel 6) und (4) wie die Agenturen von den Eigentümerressorts gesteuert werden (Kapitel 7). Außerdem hat das Evaluatorenteam einen Vergleich mit Förderagenturen in Europa durchgeführt (Kapitel 8) und drei mögliche Handlungsoptionen für die zukünftige Ausgestaltung der Beziehungen zwischen "Prinzipal" und "Agent" skizziert (Kapitel 9). Eine Beschreibung des methodischen Vorgehens (Kapitel 2) und ein abschließendes Kapitel mit Schlussfolgerungen und Empfehlungen (Kapitel 10) komplettieren den Bericht.

Zur Gewährleistung einer gendersensiblen Sprache nutzen wir im Folgenden das Binnen-I. 


\section{$2 \quad$ Methodisches Vorgehen}

Die Evaluierung basiert auf einem Methodenmix aus vier verschiedenen Datenerhebungsverfahren (Dokumenten- und Literaturanalyse, Auswertung sekundärstatistischer Daten, Interviews, Online-Befragungen), einem internationalen Vergleich, der auf Dokumentenanalysen und Telefoninterviews gründet sowie außerdem fünf Workshops, die im Evaluationszeitraum mit dem Zwecke der Validierung und Diskussion der Ergebnisse stattfanden. Aufgrund der im Statistikgesetz begründeten mangelnden Zusammenführung von Sekundärdaten in Österreich konnte jedoch keine analytisch-quantitative Abwägung in dieser Evaluierung vorgenommen werden.

\section{Dokumenten- und Literaturanalyse}

Für die Evaluierung hat das Evaluationsteam folgende Dokumente ausgewertet:

- Gesetze und Richtlinien (Förderrichtlinien des Bundes (FTI-RL, FFG-RL, etc.), Richtlinien aller Organisationen (ERP, aws, FFG), Forschungs- und Technolgieförderungsgesetz (FTFG), Strukturreformgesetz als Sammelgesetz, aws-/FFGErrichtungsgesetz) in der jeweils aktuellen Fassung,

- Mehrjahresprogramme/Jahresberichte von FFG/aws,

- interne Materialien wie interne MitarbeiterInnenbefragungen, Kundenzufriedenheitsanalysen, Wirkungsmonitoring, Strategiedokumente, Leitbilder,

- Rechnungshofberichte,

- Veröffentlichungen zur Forschungs-/Wirtschaftsförderungslandschaft in Österreich.

\section{Online Surveys}

Das Evaluatorenteam hat mehrere Online-Befragungen durchgeführt: (1) jeweils eine Erhebung innerhalb von aws bzw. FFG, die als Vollerhebung unter den MitarbeiterInnen stattfand. Gegenstand der Befragung waren Fragen zur internen Struktur und Organisation, der Unternehmenskultur, aber auch der wechselseitigen Rollenverständnisse und Zusammenarbeitsmodi zwischen den Ministerien und den beiden Agenturen. (2) Die Befragung einer Auswahl von Mitarbeiterlnnen in den Eigentümerressorts bmvit und bmwfw, um deren Wahrnehmung der Agenturen abzubilden. Der Fragebogen für die Datenerhebungen innerhalb der Agenturen und der Ministerien enthielt neben verschiedenen standardisierten Antwortvorgaben, wie dies für Online-Befragungen üblich ist, auch zahlreiche offene Angaben, um der Komplexität des Evaluierungsgegenstandes gerecht zu werden. (3) Jeweils eine Zielgruppenbefragung für die FFG und die aws, wobei für die aws ausschließlich Unternehmen, für die FFG sowohl Unternehmen als auch Forschungseinrichtungen adressiert wurden. Die Zielgruppenbefragung hatte zum Ziel 
zu überprüfen, inwieweit durch die Gründung der FFG und der aws eine Verbesserung im Bereich der Zielgruppenansprache und -koordination erreicht werden konnte und wie sich die Strukturreformen auf die (potentiellen) Förderungsnehmerlnnen ausgewirkt haben.

Die Befragungen der Agenturen und Ministerien wurden zwischen November und Dezember 2016 durchgeführt, Die Zielgruppenbefragungen fanden im Februar/März 2017 statt. Tabelle 1 gibt die Anzahl der verfügbaren Adressen pro Befragtengruppe, das Netto-Sample sowie den Rücklauf an.

Tabelle 1: $\quad$ Fallzahlen und Rücklaufquoten der Online-Surveys

\begin{tabular}{lccc}
\hline $\begin{array}{l}\text { Online-Befragun- } \\
\text { gen: Befragten- } \\
\text { gruppen }\end{array}$ & $\begin{array}{l}\text { Anzahl } \\
\text { Adressen }\end{array}$ & $\begin{array}{l}\text { Nicht erreich- } \\
\text { bar/Netto-Sample }\end{array}$ & $\begin{array}{l}\text { Anzahl Fragebögen mit min- } \\
\text { destens 50\% Beantwortung } \\
\text { (Teilnahmequote) }\end{array}$ \\
\hline FFG & 286 & $4 / 282$ & $172(61 \%)$ \\
aws & 243 & 243 & $154(63 \%)$ \\
bmvit und bmwfw & 95 & $4 / 91$ & $51(56 \%)$ \\
FFG Zielgruppe & 753 & $34 / 719$ & $260(36 \%)$ \\
aws Zielgruppe & 649 & $12 / 637$ & $192(30 \%)$ \\
\hline
\end{tabular}

Interviews (narrative und Leitfaden gestützt)

Im Rahmen der Evaluierung wurden drei verschiedene Interviewformen genutzt: narrative Interviews, Leitfaden gestützte (problemzentrierte) Interviews und Gruppeninterviews. Narrative Interviews stellen eine Spezialform des qualitativen Interviews dar, die sich in erster Linie dadurch abgrenzt, dass sie darauf abzielen, mithilfe der kontinuierlichen, chronologischen Erzählung der Interviewten eine Narration zu etablieren. Im vorliegenden Fall wurden diese Interviews in erster Linie dazu eingesetzt, eine kohärente Chronologie der Entstehung und Entwicklung der Forschungsförderungsagenturen zu entwickeln, wobei individuelle Perspektiven und Sichtweisen intakt blieben. Die entsprechend identifizierten zentralen Ereignisse und Personen dienten dann als Basis für die Entwicklung von Interviewleitfäden für entsprechend problemzentrierte Interviews, die eine Vertiefung und Objektivierung der erhobenen Informationen ermöglichten. Insgesamt wurden je 14 Interviews mit VertreterInnen aus den Eigentümerressorts und innerhalb der FFG geführt, 16 innerhalb der aws; außerdem fanden zwei Gruppeninterviews innerhalb von FFG und bmwfw mit jeweils vier Teilnehmerlnnen statt. Hinzu kamen sieben Interviews für den internationalen Vergleich, 12 Interviews mit KundInnen der FFG 
und aws sowie 10 Interviews mit österreichischen ExpertInnen für Fragen der Innovations- und Wirtschaftsförderung. In Summe hat das Evaluationsteam damit 73 Einzelinterviews und zwei Gruppeninterviews mit weiteren acht Personen durchgeführt (vgl. Tabelle 2).

Tabelle 2: $\quad$ Übersicht über die durchgeführten Interviews

\begin{tabular}{lcc}
\hline Zielgruppe & Narrativ & Leitfadengestützt \\
\hline FFG & 4 & 10 \\
aws & 3 & 13 \\
bmvit & 4 & 4 \\
bmwfw & 4 & 2 \\
Gruppeninterview bmwfw & & 4 \\
Gruppeninterview FFG & & 4 \\
FTI-ExpertInnen & 10 & - \\
Internationaler Vergleich (VINNOVA, \\
$\begin{array}{l}\text { VLAIO, KfW, Finnvera) } \\
\text { Zielgruppen/Kundlnnen }\end{array}$ & - & 7 \\
\hline
\end{tabular}

\section{Workshops}

Im Lauf der Evaluierung wurden verschiedene Workshops durchgeführt, um einerseits die gewonnenen Ergebnisse zu validieren und andererseits die Ergebnisse für alle beteiligten Stakeholder zutreffend, passgenau und relevant zu erstellen.

Neben den Abstimmungen mit dem Auftraggeber (Kick-off-Workshop, Zwischenpräsentation, Abschlusspräsentation) fand je ein Workshop mit den beiden Förderagenturen statt, um vor der offiziellen Abschlusspräsentation eine Validierung der bis dato gewonnenen Ergebnisse zu erreichen. Diese Validierungsworkshops wurden als halbtägige Veranstaltung in den Räumlichkeiten der Förderorganisationen durchgeführt. In jeder Förderorganisation nahmen ca. 15 Personen an den Validierungsworkshops teil.

\section{Fördergesellschaften in anderen Ländern}

Ziel des Vergleichs mit Fördergesellschaften in anderen Ländern war die Herausarbeitung von geeigneten Vorgehensweisen im Förderhandeln sowie im Umgang mit institu- 
tionellen Reformen. Dabei war uns bewusst, dass ein direkter Vergleich bzw. ein "benchmarking" aufgrund der Diversität der nationalen Kontexte und des abgeleiteten Förderhandelns nur begrenzt möglich ist (Technopolis 2014).

Für den internationalen Vergleich haben wir eine Kombination aus Dokumentenanalysen und ein bis zwei Telefoninterviews pro betrachtete Organisation durchgeführt. Zur Darstellung der Förderorganisationen wurde vorab ein Template erstellt, das auch als Strukturierung für die Leitfaden gestützten Interviews genutzt wurde. Wir haben vier ausländische Förderagenturen näher betrachtet, jeweils zwei mit Strukturähnlichkeit zur FFG und zwei zur aws (vgl. Tabelle 3):

Tabelle 3: $\quad$ Für den internationalen Vergleich ausgewählte Organisationen

Vergleichsorganisation FFG

VINNOVA (Schweden)

Agentschap Innoveren \& Ondernemen (VLAIO) (Flandern, Belgien)

\section{Vergleichsorganisation aws}

Finnvera (Finnland)

KfW (Deutschland) 


\section{Evaluierung der Förderagenturen}

Bei der Analyse der Förderagenturen standen drei Themenbereiche im Vordergrund:

1. interne Struktur und Organisation,

2. Unternehmenskulturen,

3. Zielgruppenerreichung.

Jeder dieser Themenschwerpunkte wurde mit einer eigenen Zielsetzung, Methodik bzw. eigenen Leitfragen adressiert. So war es Ziel der Agenturanalyse, eine Beschreibung der internen Struktur und Organisation der beiden Förderungsagenturen FFG und aws zu erstellen, d.h. mit Blick auf deren Aufbau- und Ablauforganisation, jedoch auch unter dem Blickwinkel von Formal- und Arbeitsstruktur. Geleitet wurde die Analyse von den Zielsetzungen der Strukturreformen, d.h. der Frage der Komplexitätsreduktion, verbesserten Koordination sowie Effektivität und Effizienz des Ressourceneinsatzes. Folgende Leitfragen standen im Vordergrund:

- Lagen angemessene Startbedingungen (gesetzlich, finanziell, organisatorisch) für die FFG und für die aws vor?

- Inwieweit geschah die tatsächliche Zusammenführung der Vorgängerorganisationen? Auf welchen Ebenen wirkte sich die nunmehr übergreifende Organisation aus (Stichwort: Outphasing/Effizienz der Organisation)?

- Wie stellt sich die FFG bzw. die aws in Bezug auf den Organisationsaufbau und die daraus ableitbare Organisationsdynamik heute dar (Flexibilität/Dynamik/Organisationsinnovation)?

- Wie funktioniert die interne Arbeits- und Aufgabenteilung in horizontaler (nach Förderungslogiken) und vertikaler (nach Hierarchie und internen Prozessen) Dimension?

- Wie entwickeln sich Personal- und Kostenstrukturen (insbesondere Overhead)?

- Wie entwickelt sich das Management finanzieller Risiken?

- In welchem Verhältnis sollen innerinstitutionelle Vereinheitlichung und Differenzierung sowie die Zentralisierung/Standardisierung der Prozesse (Projektcontrolling und Audit, Qualitätsmanagement, Vergabeverfahren, Instrumentenkoffer der FFG etc.) und das Belassen von Interpretationsspielräumen stehen?

- Wie hat sich die Governance durch die Zusammenführung der Vorgängerorganisationen geändert (veränderte Handlungsspielräume durch Reduktion der Akteure, neues Verhältnis zu den Zielgruppen)?

- Welche Ereignisse und Entscheidungen haben einen beobachtbaren Einfluss auf die Entwicklung der Organisationen seit der Zusammenführung gehabt?

- Wie wirken die inneren Organisationsnotwendigkeiten der FFG bzw. der aws nach außen (z.B. im Hinblick auf Koordination und Standardisierung)? 
- Wie wirkt sich die institutionelle Vereinheitlichung in Form der FFG bzw. der aws auf die Förderungsziele aus (Wechselwirkung zwischen vormals getrennten, zielgruppenspezifischen Organisationen)?

Die Analyse der Unterschiede in der Unternehmenskultur der Bereiche der FFG bzw. der aws intendierte, die zugrundeliegenden Werte, Normen und Grundannahmen, die in den jeweiligen Förderorganisationen vorliegen, zu identifizieren. Konkret standen hierzu folgende Leitfragen im Vordergrund:

- Welche Unternehmenskulturunterschiede gibt es in den einzelnen Bereichen der FFG bzw. der aws?

- Wie sind die Unternehmenskulturunterschiede zu beurteilen?

- Welches Selbst-, Fremdbild und Rollenverständnis hat sich innerhalb der FFG bzw. der aws entwickelt?

- Inwieweit geschah die tatsächliche Zusammenführung der Vorgängerorganisationen im Sinne von gemeinsamen Werten, Normen und Grundannahmen innerhalb der Bereiche der FFG bzw. der aws?

Bei der Analyse der Zielgruppenerreichung stand die Evaluierung der Wahrnehmung der Strukturreformen durch die (potentiellen) FörderungsnehmerInnen von FFG und aws im Vordergrund. Institutionen sind im Endeffekt nur so gut, wie sie von den AdressatInnen ihrer Leistungen (= KundInnen) wahrgenommen werden. Eines der Ziele, die zu den Strukturreformen im Förderungsbereich führten, war die "Erhöhung der politischen Steuerungsfähigkeit gegenüber den Zielgruppen". Die unterschiedlichen Funktionslogiken der Vielzahl an Förderungseinrichtungen schufen in Teilbereichen ein Problem der koordinierten Kommunikation mit den Zielgruppen. Die Überprüfung dessen, in wie weit durch die Gründung der FFG und der aws eine Verbesserung im Bereich der Zielgruppenansprache und -koordination erreicht werden konnte und wie sich die Strukturreformen auf die (potentiellen) FörderungsnehmerInnen ausgewirkt haben, ist Gegenstand dieses Themenbereichs. Konkrete Leitfragen hierzu waren:

- Wie gut erreicht die FFG bzw. die aws (potentielle) Förderungsnehmerlnnen im Vergleich zur Situation vor 2002 bzw. 2004?

- Wie komplex/klar wird jede der beiden Organisationen für sich wahrgenommen?

- Welche Wahrnehmung haben die FörderungswerberInnen in Bezug auf FFG bzw. aws und Ministerien? Insbesondere: Wie nehmen die Fördernehmer FFG und aws in Bezug auf deren Rollenverteilung wahr?

- Wie gut funktioniert das institutionelle Konstrukt FFG bzw. aws gemäß ihres jeweiligen Auftrags aus Sicht der FörderungswerberInnen? 


\section{Die Forschungsförderungsgesellschaft FFG}

Die FFG (Österreichische Forschungsförderungsgesellschaft) ist die österreichische Förderungsorganisation für angewandte, unternehmensnahe Forschungs- und Entwicklungsaktivitäten. Sie verfügt über ein stark ausdifferenziertes Portfolio an Förderprogrammen und -instrumenten. Ihr Aufgabenbereich umfasst "die Förderung von Forschung, Technologie, Entwicklung und Innovation zum Nutzen Österreichs" (§ 3 FFGGesetz in der Fassung vom 17.12.2014). Konkret beinhalten die Aufgaben folgende Elemente (ebd.):

"1. Förderung von Forschungs- und Entwicklungsvorhaben natürlicher und juristischer Personen,

2. Durchführung strategischer Fördermaßnahmen und -programme für FTE,

3. Förderung der Kooperation von Wissenschaft und Wirtschaft,

4. Unterstützung der österreichischen Wirtschaft und Wissenschaft in allen Belangen der Teilnahme an europäischen und internationalen Forschungs- und Technologiekooperationen,

5. Vertretung der österreichischen Interessen gegenüber den relevanten europäischen und internationalen Institutionen im Auftrag des Bundes,

6. Unterstützung des Bundes bei der Konzeption und Weiterentwicklung von FTEFörderungsmaßnahmen und -programmen,

7. Sensibilisierung der Öffentlichkeit für die Bedeutung von FTE".

Im Jahr 2016 verfügte die FFG über ein operatives Budget (Mittelwerte geglättet) in Höhe von 610 Mio. Euro (vgl. auch Abschnitt 4.2.3).

Die FFG adressiert schwerpunktmäßig Unternehmen, aber auch im steigenden Ausmaß Hochschulen und Forschungseinrichtungen sowie in geringerem Ausmaß auch EinzelforscherInnen (vgl. FFG 2014). Abgesehen von der Ausrichtung der FFG auf angewandte Forschung (industrielle Forschung und experimentelle Entwicklung), wird mit einzelnen Programmen auch der Brückenschlag zur eher grundlangenorientierten Forschung gelegt und die Überführung der Forschungsergebnisse in den Markt angestrebt. In einigen Programmen werden zudem Unternehmensneugründungen in der Form von Start-ups/Spin-offs explizit als Zielgruppe angegeben. Direkte Zuschüsse stellen die bedeutendste Form der Forschungsförderung durch die FFG dar. Die FFG vergibt jedoch im Basisprogramm neben Zuschüssen auch Darlehen und übernimmt Haftungen. Das Risiko für die Haftungsinstrumente trägt die FFG selbst, die dabei auf gute Erfahrungen im Bereich der Basisprogramme zurückblicken kann. 
Der Aufgabenbereich der FFG ist seit der Errichtung der Agentur erweitert worden, womit die FFG ein relativ großes Mandat hat: Sie übernimmt neben ihren Kernaufgaben auch die Begutachtungstätigkeit für die Forschungsprämie, das EU Performance-Monitoring, die Administrierung der Breitbandmilliarden und die Abwicklung der Klimafondsmittel.

Die Bundesministerien für Verkehr, Innovation und Technologie (bmvit) und für Wissenschaft, Forschung und Wirtschaft (bmwfw) teilen sich die Eigentümervertretung des Bundes bezüglich der FFG zu jeweils $50 \%$.

\subsection{Historie und Vorläuferorganisationen}

Die FFG ist 2004 aus der Zusammenlegung von vier bis dahin bestehenden Organisationen hervorgegangen (dem Forschungsförderungsfonds für die gewerbliche Wirtschaft - FFF, der Technologieimpulse Gesellschaft zur Planung von Technologiezentren GmbH - TIG, der Österreichischen Gesellschaft für Weltraumfragen - ASA, sowie des Büros für internationale Forschungs- und Technologiekooperation - BIT), die alle der Förderung der angewandten Forschung zugeordnet waren. Einer der Vorgänger der FFG, der FFF, ist gleichzeitig mit dem Fonds zur Förderung der wissenschaftlichen Forschung (FWF) 1967 als autonomer Fonds mit ähnlicher Logik und unter sozialpartnerschaftlicher Governance errichtet worden: hier wurde stark auf die Autonomie im Sinne der Unabhängigkeit von politischen Vorgaben geachtet.

Ein Ziel der Fusion war, dass die FFG groß und schlagkräftig genug sein muss, um international relevant sein zu können. Die Fusion selbst brauchte dann einige Zeit, berichtet wird ein Zeitraum von ca. sieben bis acht Jahren, auch wenn die Zusammenlegung der Vorgängerorganisationen relativ früh als Idee existierte.

Wesentliche Ereignisse seit der Fusion, die das heutige Agieren der FFG prägen, sind (1) die Forschungsprämienbegutachtung, die im Rahmen einer Erweiterung des existierenden FFG-Rahmenvertrags erfolgt und als Querschnittsaufgabe die Identität der FFG als eine gemeinsame Organisation stärkt; (2) der Umzug in die Sensengasse (Haus der Forschung), (3) die Einrichtung einheitlicher Prozesse, Kostenabrechnungen und eines einheitlichen Monitoring-Systems ab dem Jahr 2008 und schließlich (4) die Einführung der wirkungsorientierten Haushaltsführung ab dem Jahr 2012. Seit den Jahren 2011/2012, so die Einschätzung von GesprächspartnerInnen, könne man von der Existenz einer einheitlichen Organisation sprechen.

Insgesamt wird die FFG nun als starker, gut sichtbarer Akteur in der österreichischen Forschungs- und Innovationslandschaft angesehen mit einer allein aufgrund seiner Größe deutlich gestiegenen Bedeutung - so die einhellige Selbst- und Fremdeinschätzung. 


\section{Bewertung der Fusion}

In Summe der durchgeführten Interviews sowohl innerhalb als auch außerhalb der FFG können wir festhalten, dass die Geschäftsführung der FFG die integrierende Kraft im Fusionsprozess war. Sie stand für Kontinuität, indem sie Bewährtes belassen hat, beispielsweise das damalige FFF-Prinzip der internen Begutachtung von Einzelprojekten, aber auch die Portfoliogespräche (jetzt: FFG-Gespräche) mit Unternehmen. Die Integration der vier Vorläuferorganisationen kann als insgesamt gut gelungen angesehen werden. Zum Gelingen beigetragen hat, dass man aus dem nicht immer optimal verlaufenen Fusionsprozess bei der aws, der zwei Jahre vorher stattfand, lernen konnte. Insbesondere habe man aus den überzogenen Erwartungen bei der aws-Gründung gelernt und ist die FFG-Fusion deutlich realistischer angegangen, wovon die FFG profitierte. Zudem wurden anfängliche Befürchtungen, dass Einsparziele im Vordergrund stünden, durch das rapide Wachstum der FFG in den Anfangsjahren ausgeräumt, was insgesamt die Akzeptanz der Strukturreformen verbessert hat.

Seit 2006 ist laut Auskunft von GesprächspartnerInnen im Wesentlich klar, wer innerhalb der FFG für welche Aufgabenbereiche zuständig ist. Die Agenturstruktur wird so, wie sie seit ca. dem Jahr 2006 existiert, als funktional erlebt. Diese Einschätzung wird nicht nur von den GesprächspartnerInnen innerhalb der FFG, sondern im Großen und Ganzen auch von den FFG-KundInnen geteilt, die eine insgesamt positive Einschätzung zur Arbeitsweise der FFG abgeben (vgl. Kapitel 4.4).

\subsection{Interne Strukturen und Prozesse}

\subsubsection{Aufbauorganisation}

Die FFG ist untergliedert in sechs operative Bereiche sowie die internen Dienstleistungen und Stabsstellen. Die sechs operativen Bereiche sind: Basisprogramme, Strukturprogramme, Thematische Programme, Europäische und internationale Programme, Agentur für Luft- und Raumfahrt sowie Projektcontrolling und Audit. Zu den internen Services zählen Recht, Personal und Organisation, Finanzen, Informationstechnologien und die Öffentlichkeitsarbeit. Als Stabsstellen verbleiben Qualitätsmanagement und Interne Audits, Strategie und Datenanalysen und die Forschungsprämie (https://www.ffg.at/content/organisationseinheiten-der-ffg vom 10.01.2017).

Das Projektcontrolling und Audit ist bewusst als Querschnittabteilung angelegt, um eine profunde Kenntnis der geförderten Projekte nicht nur von der inhaltlichen Seite her zu gewährleisten, sondern auch von der abrechnungstechnischen Seite. Wesentliche Entscheidungen werden immer im Team aus MitarbeiterInnen der operativen Bereiche und 
dem Projektcontrolling getroffen, was gerade bei mehrjährigen Programmen als sehr hilfreich angesehen wird.

In der Bereichsstruktur lassen sich die Vorläuferorganisationen noch unschwer erkennen. Dem liegt einerseits eine bewusste Entscheidung zugrunde, im Rahmen der Fusion nicht über Gebühr für Unruhe zu sorgen, in dem die Vorgängerorganisationen als Referenzrahmen aufgelöst werden. Zum anderen hat sich, so die einhellige Meinung der GesprächspartnerInnen innerhalb der FFG, die Bereichseinteilung als sehr funktional erwiesen. Alle in den vergangenen Jahren unternommenen Gedankenspiele, eine für die Aufgaben der FFG bessere Organisationsstruktur zu entwickelt, haben zu keinem überzeugenden Ergebnis geführt.

\section{Abbildung 1: $\quad$ Organisationseinheiten der FFG}

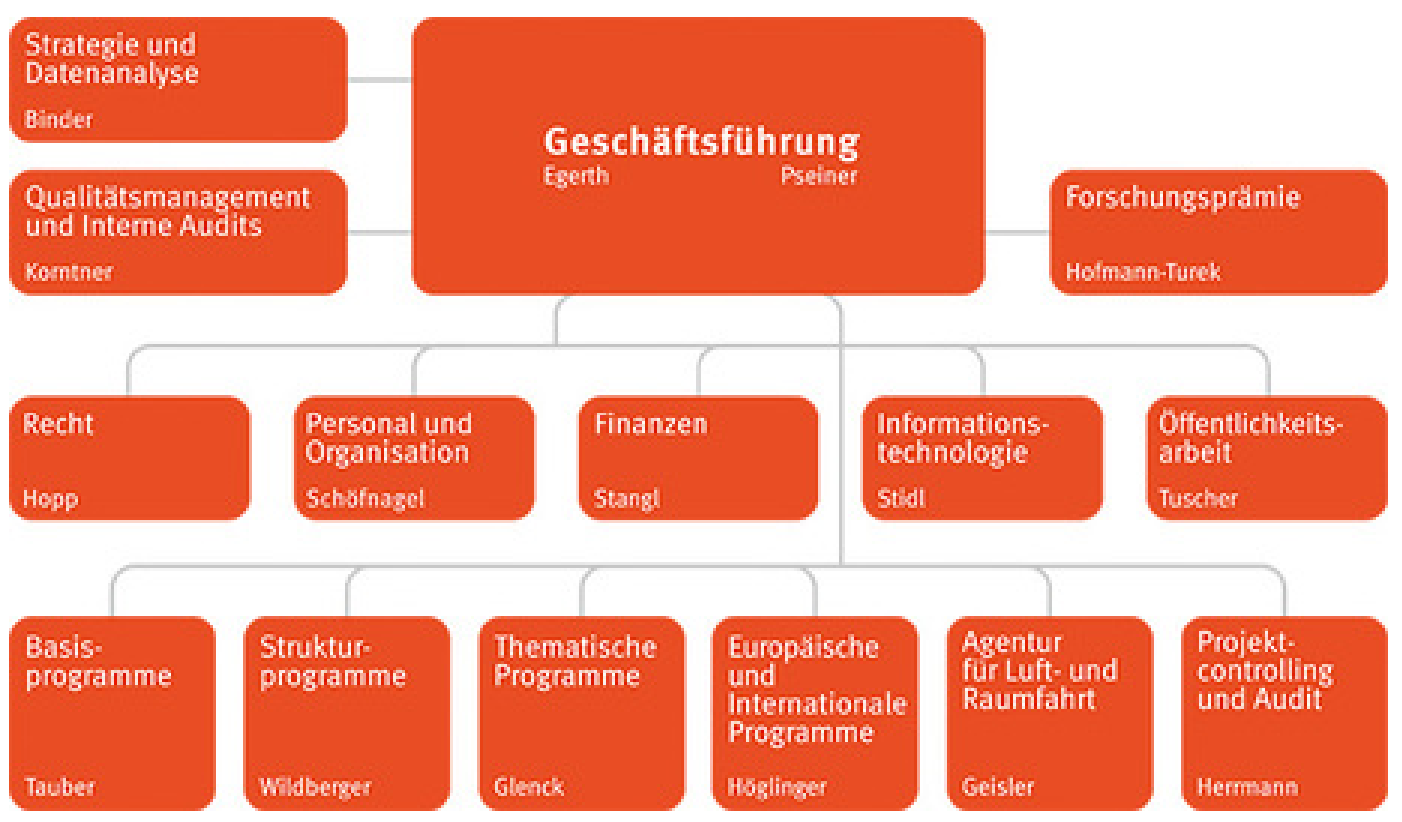

Die Stärkung der horizontalen Ebene innerhalb der FFG wird über wöchentliche jour fixes mit den BereichsleiterInnen gewährleistet, den so genannten Bereichsleiter-Treffen, an denen auch die Geschäftsführung und die Stabstellen teilnehmen. Diese Treffen gelten als sehr gut etabliert und effektiv. Es wird dort berichtet, was es Neues aus den Bereichen und wo es Schnittstellen gibt. Einmal im Monat wird dieses Treffen um die Teilnahme der internen Services erweitert. Die Abstimmung zwischen den Bereichen erfolgt außerhalb der jour fixes auch durch Abfragen, beispielsweise wer bei neu anstehenden Beauftragungen Interesse an der Bearbeitung und die notwendige Expertise für die Aufgabenerfüllung hat. 
Die internen Prozesse wie der Instrumentenleitfaden, der Prozess der Förderabwicklung und die Handhabung des eCall führen zur Stärkung einer einheitlichen Vorgehensweise. Ein aktuelles Beispiel dafür, wie die FFG mit internen Prozessen umgeht, ist die Handhabung von Evaluierungen (Mayer 2016): In einem PDF-Dokument, das dem Evaluationsteam zur Verfügung gestellt wurde, wird dort die Einbettung von Programmevaluierungen in die Prozesslandschaft dargestellt, Prozesseigner und -verantwortliche sowie Schnittstellen zu anderen Prozessen benannt, Ziele und Umfang der Prozesse definiert, der Prozessablauf festgelegt sowie eine Zusammenfassung bisher vorliegender Erfahrungen gegeben.

Die Existenz einer eigenen Strategieabteilung, die kurz nach Gründung der FFG erfolgte, wird von MinisteriumsvertreterInnen grundsätzlich positiv gesehen, weil man auf Strategieebene dadurch ein direktes Gegenüber hat. Die Strategieabteilung gilt auch als besonders hilfreich für die Positionierung im internationalen Umfeld, die für die FFG eine zentrale Rolle in ihrem Tätigkeitsportfolio einnimmt, wie sich anhand der von der FFG übernommenen Aufgaben im Rahmen von TAFTIE, dem Zusammenschluss zahlreicher Forschungsförderungsorganisationen, ablesen lässt, u.a. der Leitung der TAFTIE Akademie. Ebenfalls geschätzt wird von GesprächspartnerInnen aus den Ministerien die (strategische) Analysekompetenz dieser Stabsabteilung, die als hilfreiche Ergänzung zu den eher knappen personellen Ressourcen innerhalb der Ministerien betrachtet wird. Dass es hierdurch jedoch auch zu überlappenden Zuständigkeiten und Divergenzen in Selbstund Fremdbild kommen kann, zeigt das Kapitel 7 (Steuerung und Governance).

Das Wachstum, vor allem auch die deutlichen Budgetsteigerungen bis zu den Jahren 2009/2010 (vgl. Abschnitt 4.2.3) haben dazu geführt, dass die FFG auch mehr Verantwortung über diese größeren Budgets bekommen hat. Ab einer dreistelligen Anzahl von MitarbeiterInnen sind auch spätestens Qualitäts-Managementprozesse und Querschnittbereiche wie Recht, Personal und Strategie notwendig. Insgesamt ist die FFG, legt man das Verhältnis von personeller Ausstattung zum zu administrierenden Budget zugrunde, deutlich effizienter geworden. Seit der Fusion können, so die Einschätzung von Befragten, auch Synergien besser genutzt werden: Im Bereich Basis-Programme wird mit Blick auf die geförderten Projekte vor allem mit internen Evaluierungen gearbeitet, die Projektprüfung in wirtschaftlicher und technischer Hinsicht erfolgt innerhalb der FFG. Mittlerweile führen die MitarbeiterInnen aus dem Bereich Basisprogramme auch die gesamte Bonitätsprüfung für das Haus durch, als bereichsübergreifende Dienstleistung. Andere Bereiche werden bei fachlichen Projektevaluationen unterstützt, vor allem bei Netzwerkprogrammen. 


\subsubsection{Beauftragungssystem}

Die FFG besteht aus einem weitgehend autonomen Bereich (zurückgehend auf den ehemaligen FFF) und einem umfangreicheren, beauftragten Bereich, in dem die zuständigen Ministerien stärker Vorgaben machen. Der Bereich Basisprogramme berichtet in seiner Eigenschaft als Teil der FFG gleichwohl den zuständigen Ministerien und orientiert sich an den vom Bund vorgegebenen Richtlinien. Die Förderungsentscheidung wird aber (wie bereits beim FFF) von einem Beirat empfohlen, der sich aus Sozialpartnern und Unternehmensvertretern zusammensetzt. Bei den beauftragten Programmen liegt die Förderungsentscheidung bei den jeweils beauftragenden Ministerien. Lediglich bei kleineren Maßnahmen wie dem Innovationsscheck entscheidet die FFG-Geschäftsführung.

Von der FFG sind Mehrjahres- und Jahresprogramme zu erstellen, die durch die Eigentümer genehmigt werden müssen. Darüber hinaus wurde zwischen den Ministerien und der FFG ein Rahmenvertrag abgeschlossen, in dem z.B. die Berichtspflichten der FFG an die Ministerien (regelmäßige und standardisierte Berichte) und die Prozesse der Beauftragungen der FFG durch die Ministerien geregelt sind. Der Rahmenvertrag (bzw. dessen Beilagen) aus dem Jahr 2007 wird derzeit überarbeitet. Hierzu wurde eine Arbeitsgruppe eingesetzt, an der ca. 10 VertreterInnen der FFG, sieben des bmvit und einer des bmwfw teilnehmen. Ziel ist es, den Rahmenvertrag "an die Realität anzupassen" und möglichst flexibler zu gestalten. Dabei geht es fast ausschließlich um operative Themen wie Zeichnungsberechtigungen, Aufbereitung von Daten, expliziter Bedarf für neue Verträge bei Folgeprogrammen, Möglichkeiten zur Verschlankung etc.

Jede Beauftragung ist an einen Ausführungsvertrag geknüpft. In diesem wird u.a. die maximale Höhe der administrativen Kosten für das Programmmanagement im Verhältnis zur vergebenen Förderung festgelegt wird sowie der Umfang an Mitteln, der für die Förderung zur Verfügung steht. Außerhalb der Basisprogramme existieren somit Einzelbeauftragungen. Das bmvit ist laut FFG-Arbeitsprogramm 2016 für ca. 52\% der FTI-Mittel zuständig, das bmwfw für 11\%1.

Für die beauftragten Programme sind jeweils als Rechtsgrundlage Programmdokumente auf Basis bestehender Richtlinien oder Sonderrichtlinien zu erstellen, in denen neben dem Evaluierungskonzept auf Programm- und Projektebene jeweils Indikatoren für die Ziele der Programme definiert werden. Auch für die autonomen Programme der

1 Die restlichen Anteile verteilen sich auf FFG+ERP (12,5\%), KLIEN (7,4\%), Haftungen $(6,1 \%)$, NATS $(4,7 \%)$, Bundeslandkooperationen $(4,1 \%)$, EFRE $(1,3 \%)$ und EU $(0,4 \%)(A r-$ beitsbericht 2016, Seite 12). 
FFG auf Basis der FFG-Richtlinien sind Programmdokumente möglich, aber nicht erforderlich. In diesen Programmdokumenten sind jeweils Indikatoren definiert, anhand derer der Beitrag zu den Programmzielen überprüft werden kann.

Als Fazit können wir festhalten, dass die Arbeit der FFG durch eine Vielzahl unterschiedlicher Beauftragungsmodi geprägt ist, d.h. je nach Programm existieren unterschiedliche Steuerungsmechanismen. Neben den Beauftragungen durch die Eigentümerressorts werden von der FFG auch Mittel der Bundesländer (u.a. Oberösterreich, Tirol, Salzburg und Steiermark) administriert. Bei der Zusammenarbeit mit den Bundesländern erfolgt die Bearbeitung und Entscheidung über die Fördermittel durch die FFG. Insgesamt handelt es sich dabei um ein Volumen von ca. 50 Mio. Euro. Auch wenn nicht direkt über die Budgets zusammengearbeitet wird, steht man in einem intensiven Austausch mit den Bundesländern, über Kooperationsvereinbarungen. Außer der Mittelverwaltung für die Bundesländer erledigt die FFG auch die Verwaltung von Mitteln der EU, der Nationalstiftung (Österreichfonds) und des KLIEN-Fonds.

Aktuell werden auf der Homepage der FFG 78 aktuelle Förderprogramme aufgeführt (https://www.ffg.at/foerderungen, vgl. auch FFG-Arbeitsprogramm 2016).

Die komplexe Struktur von Einzelbeauftragungen ist nach einhelliger Meinung von InterviewpartnerInnen ein starkes Hindernis für Effizienz (siehe auch Kapitel 7 zu Steuerung und Governance).

Eine zentrale Herausforderung für eine effiziente(re) Arbeitsweise ist daher eine aus Sicht von FFG-Verantwortlichen deutliche Reduktion der existierenden Programme, vor allem da es mehrere sehr kleinteilige Programme gibt, deren Administration kaum effizient zu gestalten ist. Wie eine/r der GesprächspartnerInnen es ausdrückte, werden die KundInnen mit zu vielen differenzierten Programmen bedient, wobei die Vielfalt an Programmen nicht gerechtfertigt ist, da die Kundenstruktur im Gegenzug nicht so vielfältig ist und viele Programme eine unterkritische Größe aufweisen. Dies ist darauf zurückzuführen, dass Programme im Regelfall nicht eingestellt, sondern höchstens angepasst werden. Subjektiv betrachtet führen FFG-GesprächspartnerInnen aus, dass aus ihrer Sicht die Tendenz zu kleinen Programmen und Maßnahmen zugenommen hat, die im Regelfall auch noch sehr schnell umgesetzt werden müssten. Dass dies eine Herausforderung für die Effektivität des Gesamt-Fördersystems in Österreich ist, zeigt die Zielgruppenbefragung, die in Abschnitt 4.4 diskutiert wird. 


\subsubsection{Personal- und Kostenstrukturen}

Die FFG erhält vom Bund administrative Zuwendungen. Diese setzen sich aus den direkten Programm-Zuwendungen und den programmunabhängigen Zuwendungen (=FFG-Overhead) zusammen. Durch diese Teilkostenrechnung gegenüber dem Bund ist die Höhe der Overheadkosten transparent und kann vom Bund gezielt beeinflusst werden (z.B. über 20\%-Vorgabe in Eigentümer-Strategie). Der Overhead, d.h. die Kosten für die allgemeinen Aufgaben der FFG, werden je hälftig zwischen den beiden Eigentümerressorts aufgeteilt, nur der Overhead, der im Rahmen bestimmter Beauftragungen erfolgt, wird anders berechnet. Gegenüber Dritten, beispielsweise dem Klimafonds oder der Nationalstiftung, erfolgt eine Vollkostenrechnung. Mittels eines Verrechnungssystems ist sichergestellt, dass es zu keiner Doppelverrechnung kommt.

Seit dem Jahr 2006 sind, von wenigen kleineren Abweichungen abgesehen, sowohl die operativen Mittel als auch der korrespondierende Personalaufwand kontinuierlich gestiegen (vgl. Abbildung 2), die Personalmittel jedoch langsamer als die operativen Mittel; letzteres ist ein Umstand, der von den MitarbeiterInnen durchaus kritisch gesehen wird aufgrund der damit einhergehenden Überlast. Für den operativen Bereich stehen im Jahr 2016 ca. 219 Vollzeitäquivalente (VZÄ) zur Verfügung, insgesamt 262 VZÄ.

Setzt man die administrativen Kosten (Vollkosten) der Förderprogramme zu den operativen Mitteln (geglättete Mittelwerte2) in Bezug, so zeigt sich, dass sich diese seit 2006 von damals 3,7 \% auf nunmehr 3,5\% verringert haben. Einer der Gründe für die Reduktion der Abwicklungskosten (=Programmkosten plus anteilige Overheads), neben Effizienzsteigerungen durch einheitliche Prozesse, sind die zahlreichen Neuverträge für das Personal, die im Regelfall für die Beschäftigten weniger günstige Konditionen enthalten als die Altverträge.

2 Um eine realistische Messgröße der operativen Mittel mit Bezug zum tatsächlichen Arbeitsanfall zu haben, wurde der gewichtete Mittelwert gebildet und danach geglättet. Der Mittelwert setzt sich anteilig aus Mittelbindung (10\%), Zusagen (60\%) und Auszahlungen (30\%) zusammen. Der geglättete Mittelwert wird als Durchschnitt von jeweils zwei Jahren ausgewiesen. Breitband wird derzeit noch anders berechnet, soll ab 2018 aber auch nach diesem Schema ermittelt werden. Quelle für die EU-Mittel ist die EU-Dokumentation der Auszahlungen für das Rahmenprogramm (Expenditure), Quelle für die ESA-Mittel ist ein den österreichischen ESA-Delegierten zur Verfügung gestellter Datensatz. Der geglättete Indikatorwert wird als Durchschnitt von jeweils zwei Jahren ausgewiesen. Forschungsprämie: Für 2013 wurde ein Schätzwert herangezogen, da es noch kein vollständiges in der FFG abgewickeltes Jahr war. Der geglättete Indikatorwert ab 2014 wird als Durchschnitt von jeweils zwei Jahren ausgewiesen. 
Abbildung 2: $\quad$ Entwicklung der operativen Mittel und Personalausstattung (2006-2016) (Angaben der FFG)

\section{FFG Personal- und Mittelübersicht}

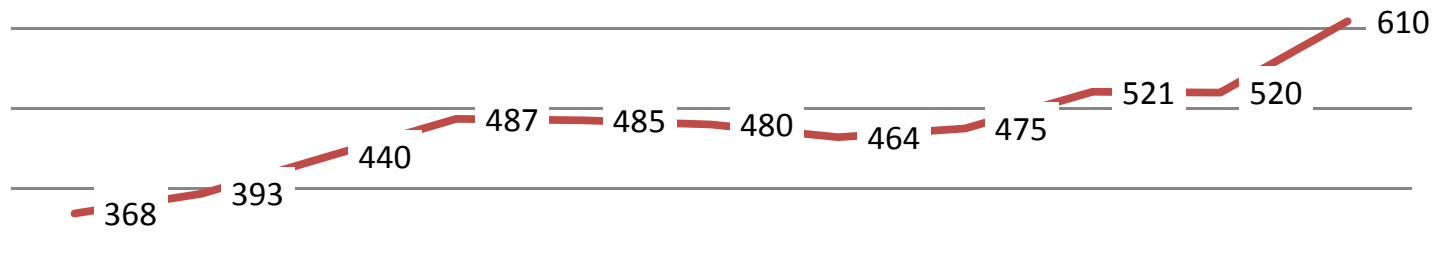

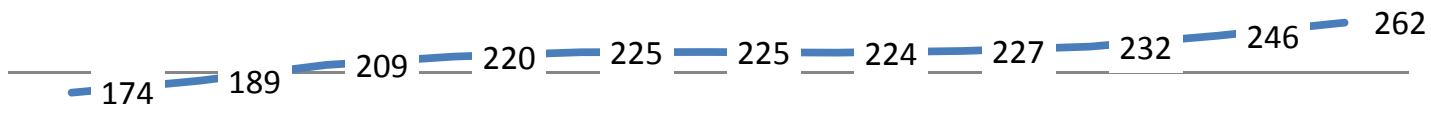

$\begin{array}{lllllllllll}19 & 20 & 22 & 24 & 24 & 24 & 26 & 26 & 27 & 29 & 32\end{array}$

$=3,7 \%=3,7 \%=3,6 \%=3,3 \%=3,3 \%-3,5 \%=3,6 \%-3,6 \%=3,4 \%-3,4 \%-3,5 \%$

$\begin{array}{lllllllllll}2006 & 2007 & 2008 & 2009 & 2010 & 2011 & 2012 & 2013 & 2014 & 2015 & 2016\end{array}$

VZÄ FFG-gesamt

Operative Mittel: Mittelwert geglättet (in Mio. EUR)

Kosten der Organisationseinheiten nach interner Leistungsverrechnung (FFG Gesamt, in Mio. EUR)

ADMINISTRATIVE KOSTEN (Vollkosten) Förderprogramme / OPERATIVE MITTEL: Mittelwert geglättet

Quelle: Angaben der FFG

Eine zentrale Herausforderung für das Agieren der FFG ist der mit den (Einzel-) Beauftragungen einhergehende Mangel an finanzieller Flexibilität. Auch Mittelumschichtungen sind aufgrund der Vielzahl an gleichzeitig zu bedienenden Ausführungsverträgen mühsam, wenn nicht sogar ganz unmöglich, da beispielsweise keine Mittel von einem ITProgramm auf ein Energie-Programm umgeschichtet werden können. Dies erschwert die strategische Themensetzung. Um hier in Zukunft flexibler agieren zu können, so die einhellige Einschätzung der GesprächspartnerInnen in der FFG, wäre ein einheitlicher Ausführungsvertrag hilfreich, kombiniert mit mehrjährigen (Global-)Budgets. In einer idealen Welt erhielte man als Förderorganisation dann klare Wirkungsziele, ein Mehrjahresbudget und anschließend die Freiheit zur Instrumentenentwicklung.

Die FFG hat außerhalb der Mittel, die von den beiden Eigentümerressorts beauftragt bzw. gewährt werden, kaum eigene Einnahmen. Rückflüsse aus gewährten Darlehen 
gehören ihr, deren Verwendung ist wiederum an die förderrechtlichen Bedingungen des Bundes geknüpft.

Wie oben bereits erwähnt, hat die FFG ihr Personal kontinuierlich, wenn auch langsam, ausgebaut. Insbesondere die thematischen Programme sind seit der Fusion im Jahr 2004 quasi vollständig neu aufgebaut worden und weisen außerdem die Besonderheit auf, dass dort nicht nur "interne" MitarbeiterInnen arbeiten, sondern auch MitarbeiterInnen des bmvit, die formal über die FFG angestellt sind. Aus Gründen der Übersichtlichkeit werden diese in die Abbildung jedoch nicht aufgenommen. Im Jahr 2016 entfallen auf diese Gruppe der externen TP-MitarbeiterInnen ca. 7 VZÄ. In der Abbildung aus Gründen der Übersichtlichkeit ebenfalls nicht enthalten sind die VZÄ für die Forschungsprämie (in 2016 insgesamt 5,23 VZÄ) und DA (in 2016 insgesamt 5,36 VZÄ).

Abbildung 3: $\quad$ Entwicklung der Vollzeitäquivalente nach Bereichen (2006-2016)* (Angaben der FFG)

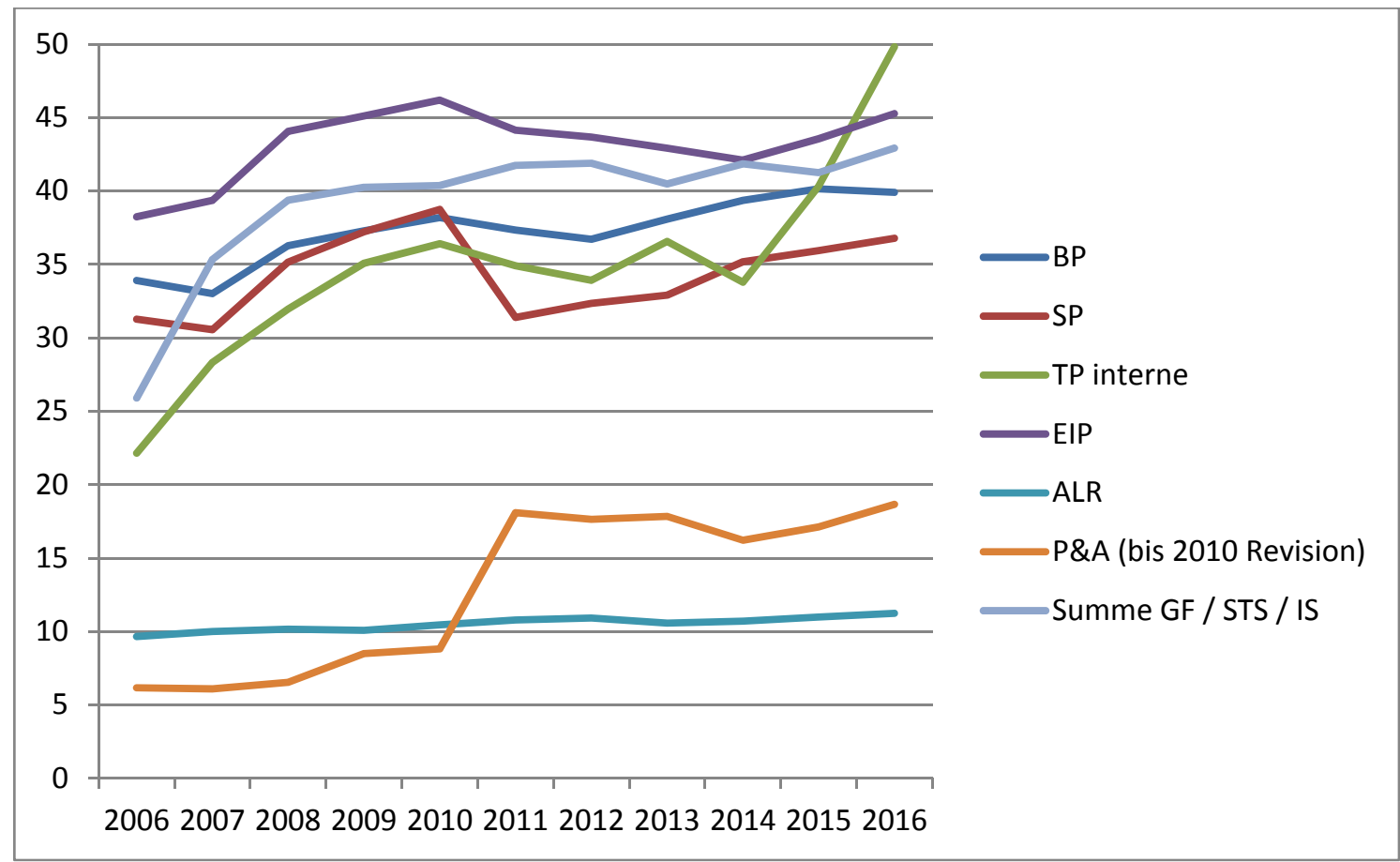

*) Die Werte sind im Jahresdurchschnitt angegeben, nicht zum Stichtag! D.h. z.B. eine Person mit 38,5 Wochenstunden, die im Juli eingestellt wurde, entspricht 0,5 VZÄ. Die Unterteilung in Bereiche/operative STS (Stabstellen) einerseits und GF (Geschäftsführung) /STS (Stabstellen) /IS (Interne Services) entspricht der aktuellen Abteilungsstruktur. Die Abteilungsstruktur ist seit 2007 mit der aktuellen vergleichbar, 2006 waren Aufgaben die ab 2007 von den IS/STS zentral übernommen wurden, noch vielfach dezentral angesiedelt. Im Jahr 2011 wurde die Revision in die Abteilungen Projektcontrolling und Audits einerseits und Qualitätsmanagement und interne Audits andererseits getrennt. Das Team, das die Projektprüfungen in SP durchgeführt hatte wurde in die Abteilung Projektcontrolling und Audit (P\&A) eingegliedert. 
Die Querschnittbereiche haben sich in ähnlicher Weise entwickelt, mit Ausnahme der ALR, deren Personalausstattung ohne Ausschläge nach oben bzw. unten kontinuierlich leicht gestiegen ist. Insgesamt fällt auf, dass nach einem deutlicheren Aufwuchs zwischen den Jahren 2006 und 2010 zunächst eine Personalreduktion stattfand, die erst in den vergangenen beiden Jahren wieder kompensiert wurde.

Ein wichtiges Instrument des strategischen Personalmanagements ist die nach der Fusion neu eingeführte strukturierte Weiterbildung, d.h. Qualifizierung wird nicht nur bedarfsweise betrieben, sondern es ist explizit gewünscht, dass Qualifizierungen stattfinden.

Die FFG führt regelmäßig Mitarbeiterlnnenbefragungen durch (ca. alle zwei bis drei Jahre), mit insgesamt sehr positiven Ergebnissen: Vor allem die Bewertung der Führungskräfte, die Klarheit der Leistungserwartungen, die Arbeitszeitflexibilität und die gelebte Gleichberechtigung ragen positiv heraus (IFES 2016). Auch in der vom Fraunhofer ISI im Rahmen der gegenständlichen Evaluierung durchgeführten Befragung finden sich hohe Zufriedenheitswerte, aber an der einen oder anderen Stelle durchaus auch Kritik, wie in den folgenden Abschnitten näher ausgeführt wird.

Basierend auf aktuellen Personaldaten (Stand: Herbst 2016) hat das Evaluationsteam eine Gesamtbefragung aller MitarbeiterInnen durchgeführt. Insgesamt wurden 286 Personen angeschrieben, von denen vier zum Zeitpunkt der Befragung nicht erreichbar waren. An der Befragung teilgenommen haben 172 Personen, definiert als diejenigen, die mindestens die Hälfte der Fragen beantwortet haben. Dies entspricht einer Rücklaufquote von $61 \%$.

Zur Struktur der Befragten ist anzumerken, dass mehrheitlich Personen aus dem Bereich "Thematische Programme" geantwortet haben, die auch insgesamt den größten Beschäftigtenanteil ausmachen. Der Bereich europäische und internationale Programme ist dagegen leicht unterrepräsentiert. Als Aufgabenschwerpunkt bezeichnet knapp die Hälfte der Befragten das nationale Programm-Management. Fast zwei Drittel der Befragten ordnen sich dem Status "Expertln" zu, aber auch Teamleitung und Assistenz sind im Befragungssample relativ häufig vertreten.

Basierend auf unserer Ausgangshypothese, dass die Beschäftigung in einer der vier Vorläuferorganisationen nach wie vor die normativen Werte und Überzeugungen prägt, haben wir in der Befragung auch erhoben, ob und wenn ja, in welcher der Vorläuferorganisationen die Befragten beschäftigt waren. Dies ist für ca. ein Drittel der Befragten der Fall. Besonders viele waren beim BIT beschäftigt, gefolgt vom FFF. 


\subsubsection{Ablauforganisation}

Wie die Interviews, aber auch Daten der FFG-internen Mitarbeiterlnnenbefragung gezeigt haben, ist die MitarbeiterInnenzufriedenheit generell als hoch einzuschätzen. Betrachtet man einzelne Prozesskomponenten, die die MitarbeiterInnenzufriedenheit beeinflussen, so zeigt sich ein heterogeneres Bild: Positiv hervorzuheben ist, dass sich eine klare Mehrheit über die zentralen Leistungserwartungen gut informiert fühlt. Auch mit der Einbeziehung in Entscheidungsprozesse sind die FFG-MitarbeiterInnen im Großen und Ganzen zufrieden, gleiches gilt für die nachvollziehbare Delegation von Verantwortung. Ausbaufähig hingegen sind die Honorierung von Verbesserungsvorschlägen sowie die Transparenz von Aufstiegskriterien (vgl. Abbildung 4). Auch die FFG-interne MitarbeiterInnenbefragung hatte die Frage nach offener Kommunikation und Feedback, die strukturähnlich zu Verbesserungsvorschlägen ist, als eher kritischen Punkt herausgearbeitet.

Abbildung 4: $\quad$ Bewertung der internen Strukturen und Prozesse der FFG

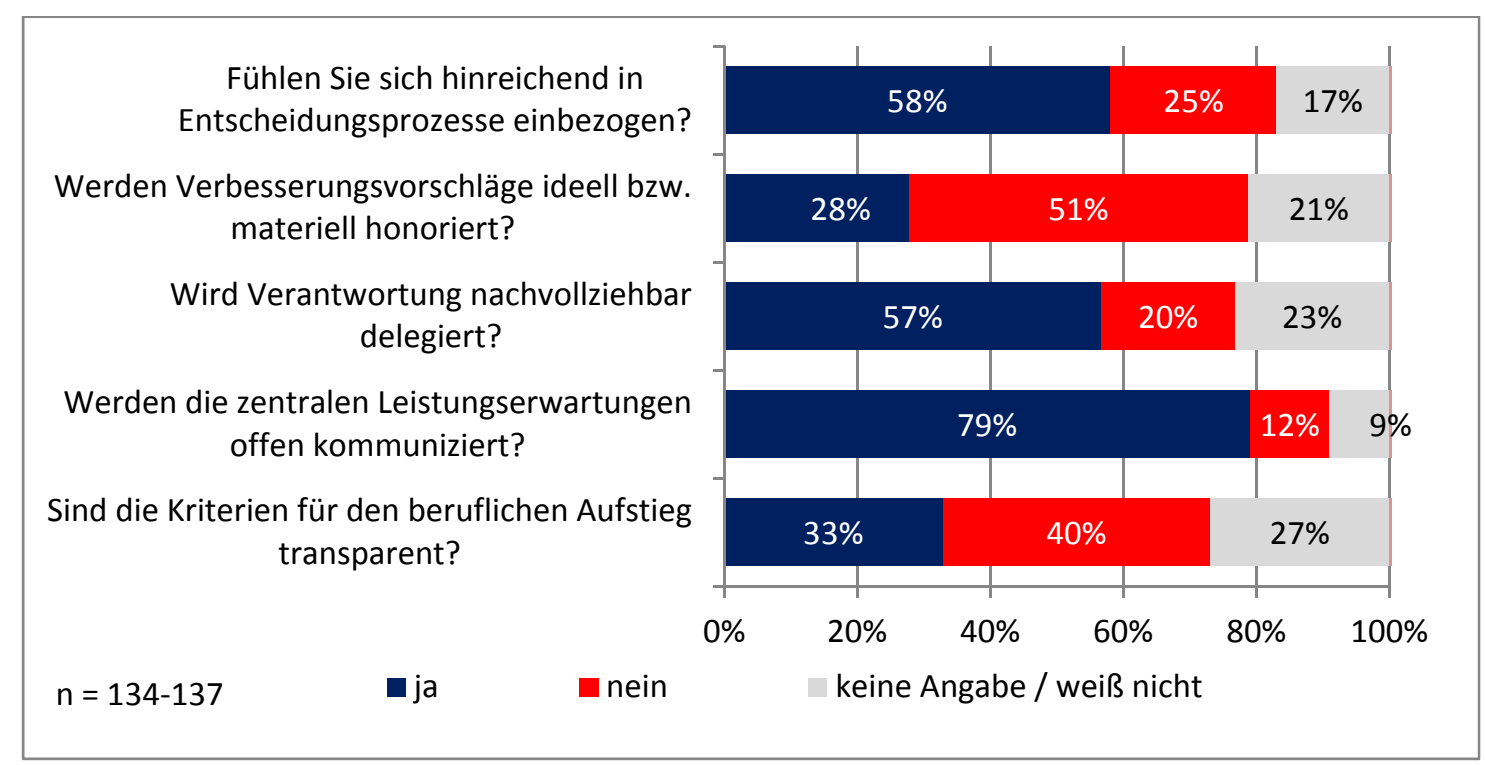

Analysiert man diese Ergebnisse nach den einzelnen Befragtengruppen, so lässt sich festhalten, dass es vor allem der Personenkreis aus dem nationalen Programm-Management ist, bei dem eine gewisse Unzufriedenheit mit den oben genannten Dimensionen vorliegt. Die Befragungsergebnisse nach Vorläuferorganisation sind unauffällig.

Eine wichtige Grundlage für die Identifikation von MitarbeiterInnen mit der Organisation, in der sie tätig sind, sind Fragen der Akzeptanz und Wertschätzung. Hier erreicht die FFG ausgezeichnete Werte, wie Abbildung 5 veranschaulicht. Dabei werden eine hohe Akzeptanz und Wertschätzung am häufigsten von Befragten mit Leitungsfunktion berichtet, am wenigsten häufig von Befragten aus dem nationalen Programm-Management. 
Abbildung 5: $\quad$ Zufriedenheit mit dem Arbeitsumfeld

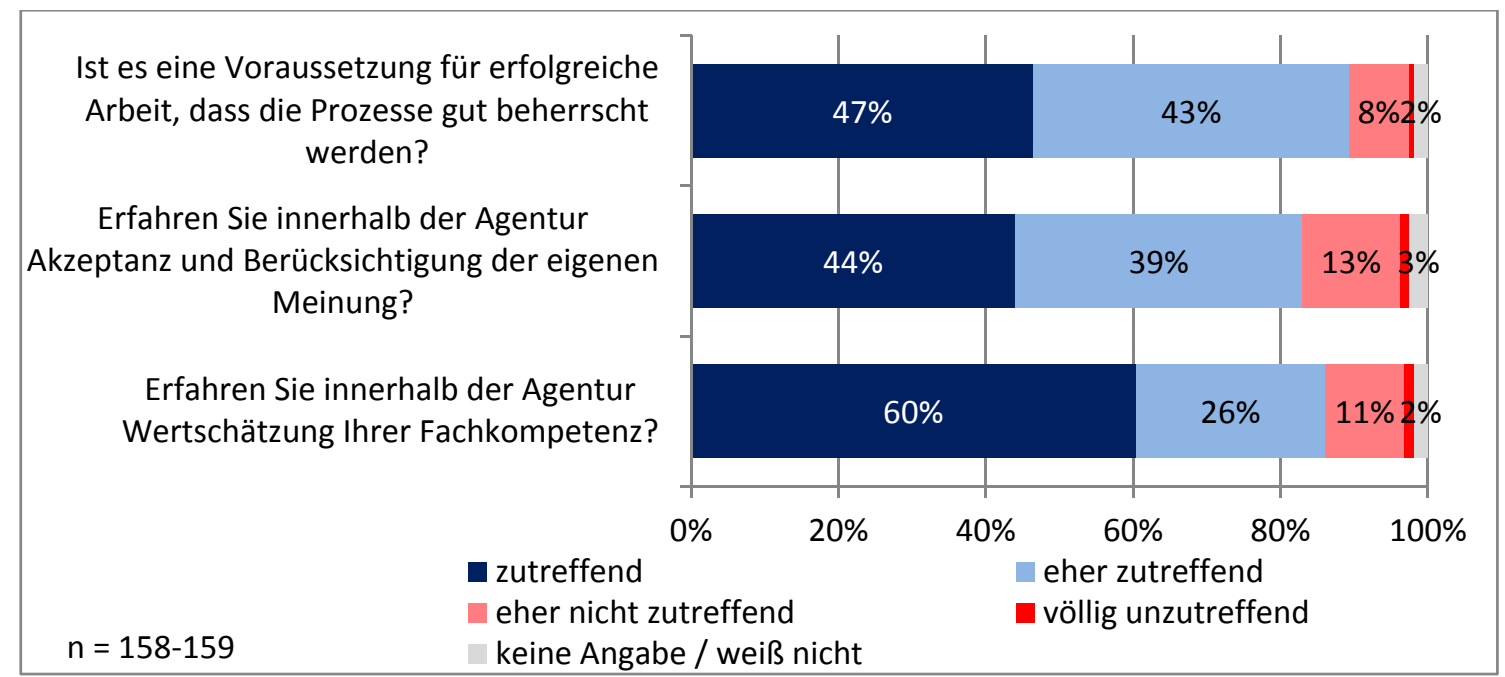

Die starke Orientierung an (standardisierten) Prozessen ist ein Merkmal, das die FFG insgesamt auszeichnet und prägt. Die Standardisierung der Prozesse fußt laut Auskunft von GesprächspartnerInnen auf einer Top-down-Strategie der Geschäftsführung, als klarer Auftrag, sich als eine einheitliche Organisation zu präsentieren und damit Berechenbarkeit und Vergleichbarkeit sicherzustellen. Das Prozessmanagement wurde als Weg dorthin begriffen, wobei Fragen der Effizienz, Wirtschaftlichkeit und des Qualitätsmanagements im Vordergrund standen. Eine zentrale Rolle hierbei haben übergeordnete und abgestimmte Dokumente gespielt. Man habe die Instrumente jedoch gemeinsam entwickelt, der Abstimmungsbedarf sei dadurch geringer geworden.

Eine Herausforderung für die FFG (wie auch für andere Förderagenturen) ist gleichwohl, wie man sich im Spannungsfeld zwischen einerseits der Gewährleistung einer hohen Effizienz und Nachvollziehbarkeit der Prozesse und andererseits Flexibilität und Eigenverantwortlichkeit positioniert. Derzeit schlägt das Pendel zugunsten effizienter Prozesse und Strukturen aus, wie auch der Rechnungshof in seinem gegenüber der FFG sehr positiven Gutachten betont (Rechnungshof 2015). Die hohe Regelungsdichte bedeutet jedoch für die Beschäftigten, dass es an Interpretationsspielräumen mangelt. Auch wird der Umfang und die Komplexität der existierenden Regelwerke und Prozessbeschreibungen von einigen der GesprächspartnerInnen in der FFG durchaus als herausfordernd gesehen. Schlussendlich, so eine pointierte Meinung aus einem Interview, werde die FFG wie ein Amt geführt, was zur Aufgabe von Forschungsförderung, zumindest partiell auch risikobehaftetere Vorhaben zu fördern, in Widerspruch steht. Die derzeitigen Rahmenbedingungen ließen eine solche Flexibilität und mutigere Entscheidungen aber nicht zu. Insbesondere die internen Kontrollsysteme (IKS) werden als sehr aufwändig erlebt. 
Die Prozessorientierung ist maßgeblich der Größe der FFG geschuldet: Eine Organisation mit knapp 300 MitarbeiterInnen und einem umfangreichen Budget zieht eine hohe Regulationsdichte und Anforderungen an ein anspruchsvolles Risiko- und Qualitätsmanagement nach sich. Gleichzeitig sind diese Strukturen aber auch Ausdruck einer durch die Rahmenbedingungen evozierte Strategie der Fehlervermeidung.

\subsubsection{Projektauswahlkriterien}

Die unterschiedlichen Aufgabenstellungen und Beauftragungen prägen den Alltag der FFG-MitarbeiterInnen und wirken sich auch direkt auf die Wahrnehmung relevanter Auswahlkriterien für Förderentscheidungen aus. Die Befragten sind dabei einhellig der Meinung, dass es bei den Förderentscheidungen primär um die Stärkung des österreichischen Forschungs- und Innovationsstandorts geht, dicht gefolgt von der Adressierung zentraler gesellschaftlicher Herausforderungen und hohen Chancen auf Marktumsetzung.

\section{Abbildung 6: $\quad$ Förderkriterien aus Sicht der FFG-Mitarbeiterlnnen}

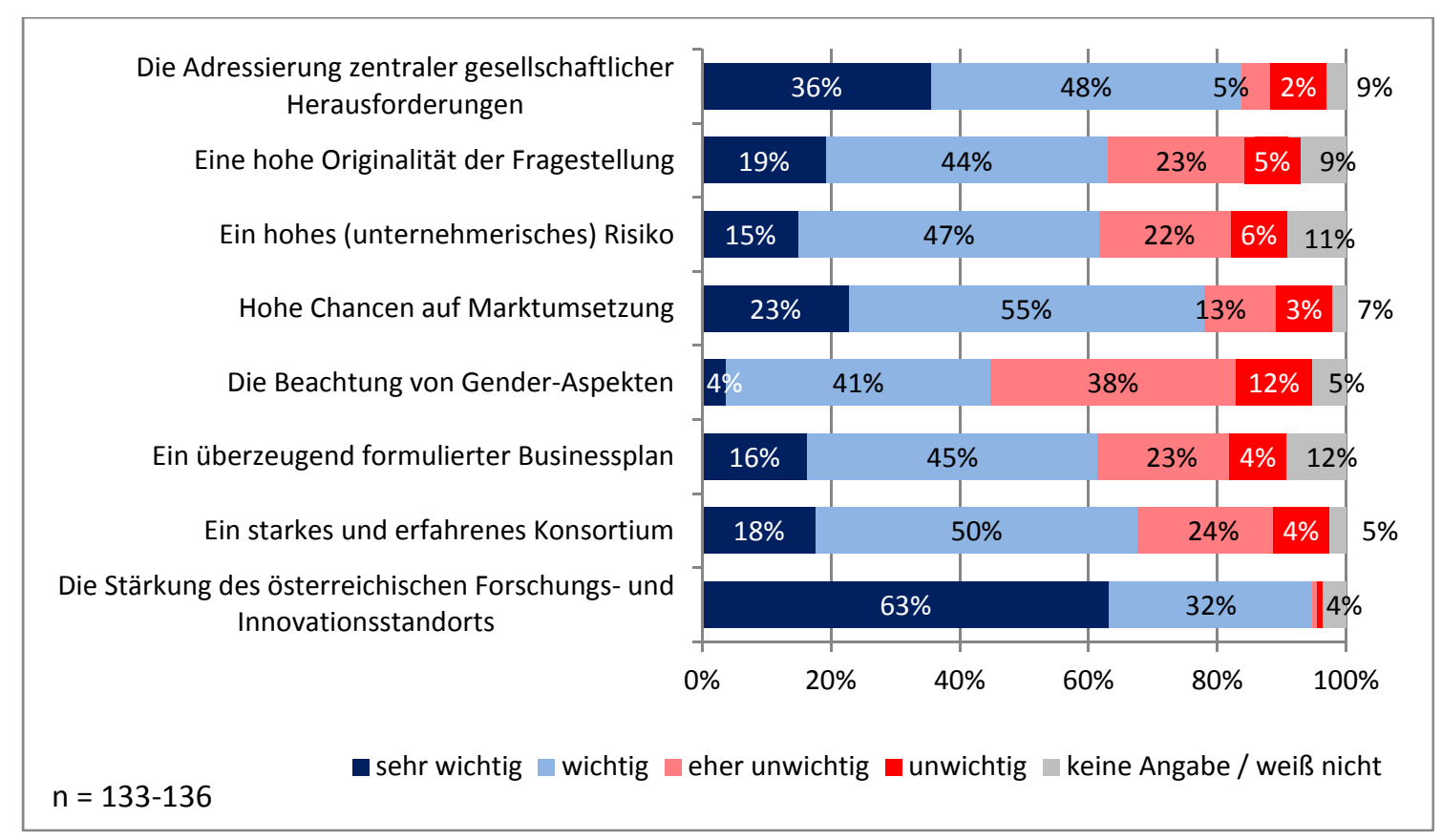

Stellt man den Perspektiven der FFG-MitarbeiterInnen nun die Sichtweise der Eigentümerressorts gegenüber, so wird deutlich, dass diese größtenteils konvergieren, mit einer wichtigen Ausnahme: ein überzeugend formulierter Businessplan hat ein signifikant höheres Gewicht aus Perspektive der Befragten in den Ministerien. 
Abbildung 7: $\quad$ Förderkriterien aus Sicht der Ministerien

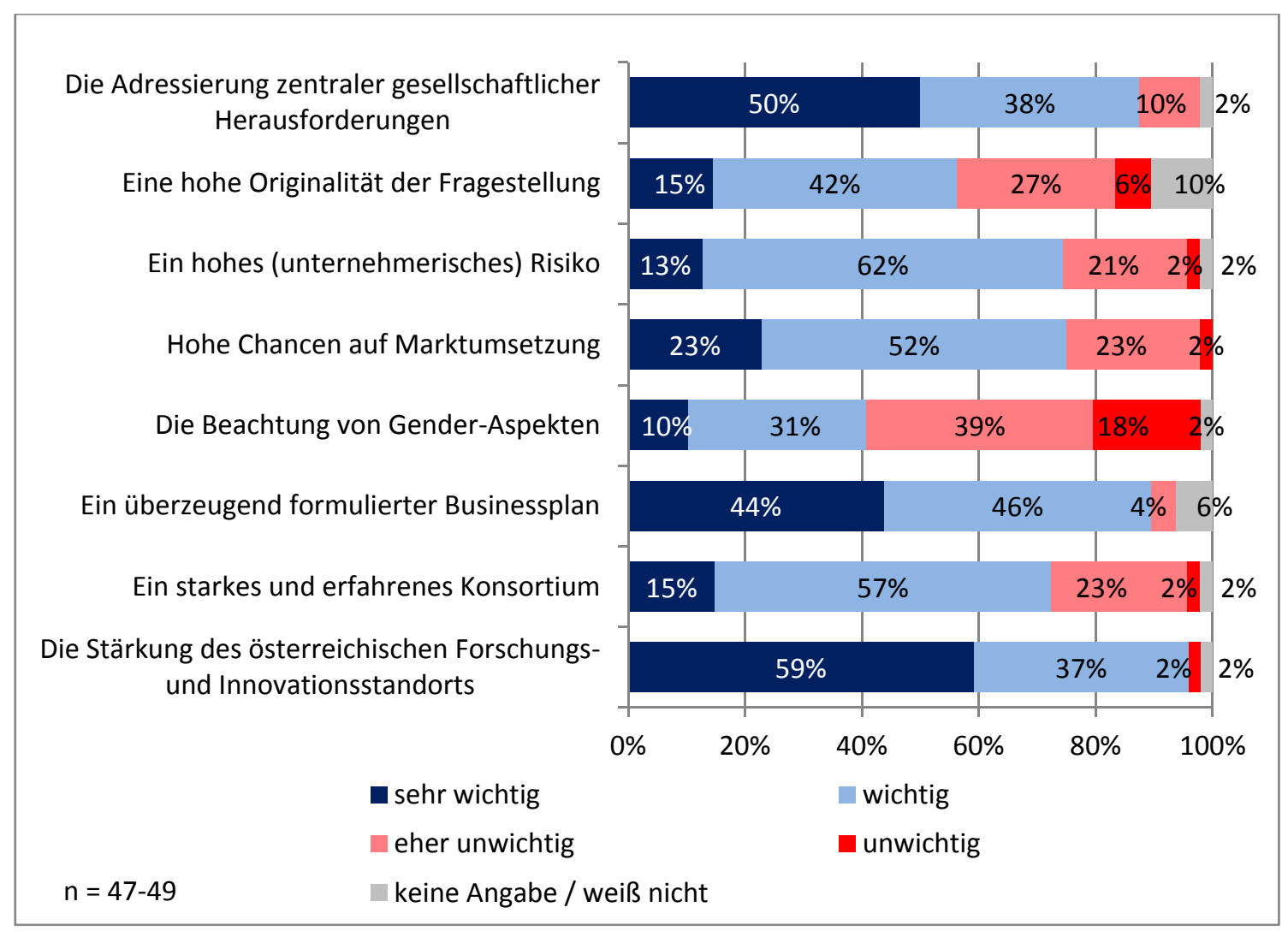

Bei der Frage der Auswahlkriterien unterscheiden sich die einzelnen Befragtengruppen: Für die Basisprogramme hat die Originalität der Fragestellung eine überdurchschnittlich große Bedeutung, auch das unternehmerische Risiko und ein überzeugender Businessplan werden hier höher gewichtet als in den anderen FFG-Bereichen. Demgegenüber spielen Aspekte des Konsortiums eine weniger wichtige Rolle, im Gegensatz zu den thematischen sowie den europäischen und internationalen Programmen, wo die Zusammensetzung des Konsortiums ein wichtiges Förderkriterium darstellt. Die Strukturprogramme weisen als Besonderheit auf, dass dort die Gender-Aspekte einen vergleichsweise hohen Stellenwert haben. Unterschiede nach Vorläuferorganisationen bestehen hingegen kaum, hier zeigt sich, dass das aktuelle, an Prozessen und Standards orientierte Handeln zu einer innerhalb der Bereiche doch recht einheitlichen Vorgehensweise führt. 


\subsection{Unternehmenskultur}

Bei der Frage nach der Unternehmenskultur sind wir drei verschiedenen Fragestellungen nachgegangen:

1. Wofür steht die Agentur aus Sicht der Befragten?

2. Welche Adjektive assoziieren sie mit der FFG?

3. Was sind die normativen Erwartungen, d.h. das "Soll" der Tätigkeiten, die innerhalb der FFG ausgeführt werden?

Zunächst: Es gibt nicht die eine FFG, sondern die Arbeitsweisen und Kulturen unterscheiden sich nach den Organisationsbereichen, also den Strukturprogrammen, den Basisprogrammen, den thematischen Programmen, den europäischen und internationalen Programme sowie der ALR, jedoch in geringerem Ausmaß, als vom Evaluatorenteam zunächst vermutet. Dies haben auch die Interviews so bestätigt: trotz gelegentlich noch zu beobachtenden Fortwirkens der Vorgängerkulturen agiert die FFG mittlerweile eindeutig als ein Unternehmen.

Betrachten wir zunächst die Befragungsergebnisse zur Frage nach den Orientierungsmaßstäben, so zeigt sich hierin das stark Prozess-orientierte und absichernde Vorgehen, vor allem Dinge gut zu dokumentieren und möglichst fehlerfrei zu bearbeiten. Dies deckt sich mit den oben genannten Ergebnissen, wonach es relativ wenige Freiheitsgrade und Raum für Kreativität gibt.

Abbildung 8: $\quad$ Normative Anforderungen in der FFG

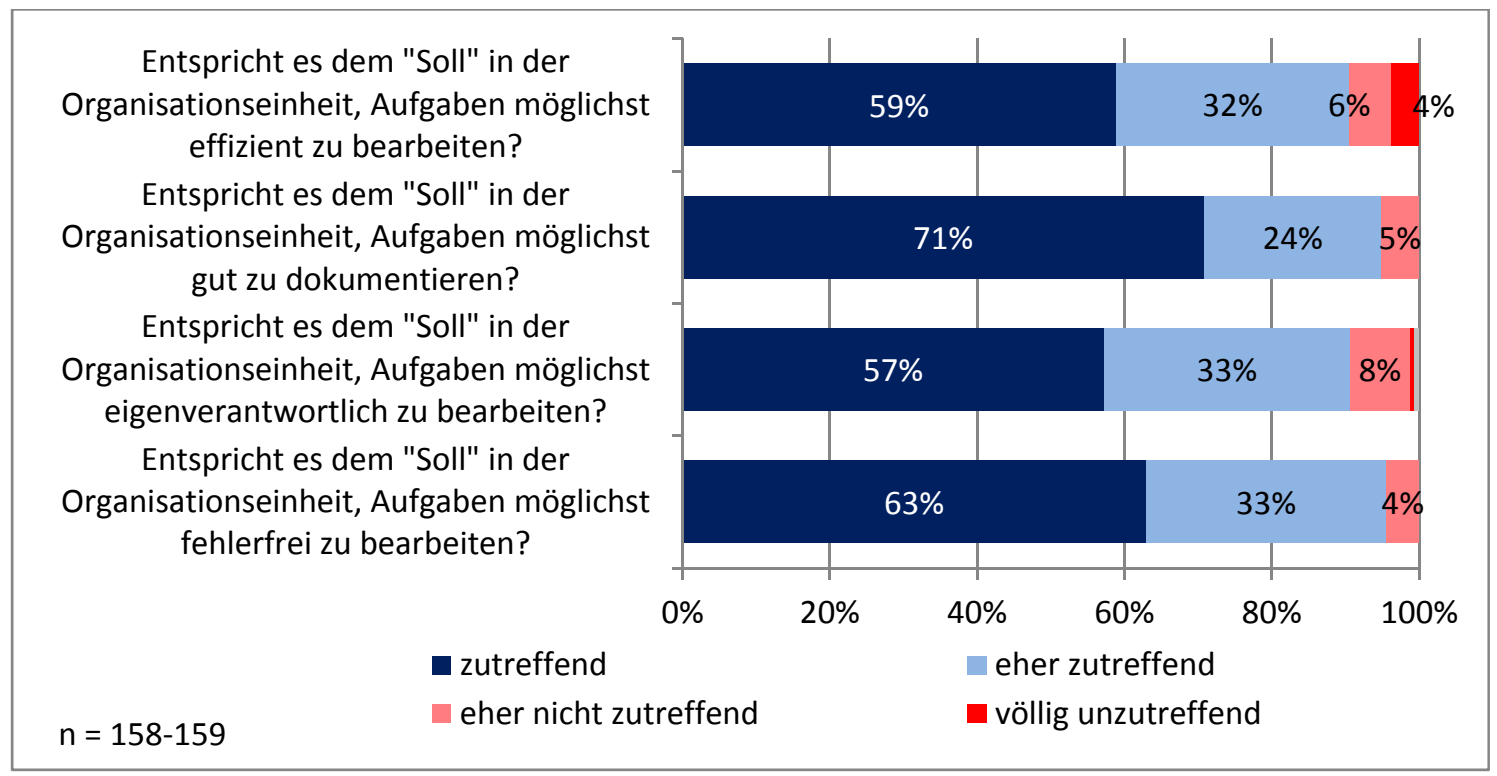


Letzteres, d.h. eine gewisse Unzufriedenheit mit dem hohen Standardisierungsgrad der Arbeiten und in Folge eher geringen Freiräumen ist auch einer der Hauptgründe für das Verlassen der FFG, wie aus den systematisch geführten Ausstiegsgesprächen hervorgeht. Auch in der FFG-MitarbeiterInnenbefragung ist die Aussage "Ich würde mir in meinem beruflichen Alltag mehr Abwechslung wünschen, einmal etwas inhaltlich Neues machen" diejenige, die den stärksten Optimierungsbedarf induziert (IFES 2016). Grundsätzlich ist die Fluktuationsrate in Höhe von ca. $4 \%$ jedoch gering.

Die große Bedeutung einer guten Dokumentation der Arbeiten wird vor allem von Personen mit Leitungsfunktion betont, am seltensten im Bereich internationales ProgrammManagement. Überhaupt wird von Leitungspersönlichkeiten das jeweilige in der Befragung thematisierte "Soll" am stärksten bekräftigt. Eine hohe Eigenverantwortlichkeit des Arbeitens wird zudem von den MitarbeiterInnen aus den europäischen und internationalen Programmen hervorgehoben. Betrachtet man die wahrgenommenen Orientierungsrahmen nach Bereichen, so betonen vor allem Befragte aus den Strukturprogrammen, dass sie ihre Aufgaben möglichst fehlerfrei bearbeiten und gut dokumentieren sollen. Für die Basisprogramme steht dagegen eine hohe Eigenverantwortlichkeit im Vordergrund.

Betrachtet man die Befragungsergebnisse im Hinblick darauf, welches Selbstbild die Befragten von der FFG zeichnen, so dominiert die Beschreibung "Fördereinrichtung von (angewandter/wirtschaftsnaher) Forschung" (118 Angaben), d.h. die Befragten geben mehr oder weniger eine Umschreibung des Akronyms an. An zweiter Stelle leiten die Befragten das Selbstbild der Agentur aus deren Zielsetzung ab, konkret der Förderung von Unternehmen und dem Wirtschaftsstandort Österreich (55 Angaben). Es folgt eine mittlere Gruppe von jeweils knapp unter 30 Nennungen, die "Förderung von Innovation", "Kunden- und Serviceorientierung" sowie "Internationalität (EU-Programme, Bindeglied zu internationalen Organisationen, Netzwerken, Gremien; internationale Projekte) als aussagekräftige Beschreibung der FFG anführen. Weitere Nennung entfallen auf "Expertise/Know-how/Kompetenz (20)", Professionalität (18), Transparenz (10), Engagement (3) sowie Effizienz, Effektivität, Wirksamkeit (2). Geben die offenen Angaben zur Frage, wofür die Agentur steht, ein erstes Bild zur Selbstverortung ab, so zeigen die aus den assoziierten Adjektiven generierten word clouds, dass sechs Begriffe die FFG aus Sicht der MitarbeiterInnen am besten beschreiben: kundenorientiert, serviceorientiert, professionell, offen, kompetent und freundlich (vgl. Abbildung 9).

Aufschlussreich ist, dass die Angaben sich zwar nicht nach Bereichen, aber sehr wohl nach Vorläuferorganisationen unterscheiden, d.h. hier überdauern kulturelle Prägungen und Sozialisierungen, die ursprünglich einmal internalisiert wurden und den Fusionspro- 
zess überdauert haben. Dies haben auch Interviews so bekräftigt, d.h. unter den MitarbeiterInnen, die auf eine langjährige Erfahrung in der Projektadministration zurückblicken können, haben sich durchaus gewisse Rollenverständnisse und Gewohnheiten erhalten, wie beispielsweise eine starke Prozess- und Regelorientierung bei ehemaligen TIG-Beschäftigten (auch die Stichworte korrekt bzw. überkorrekt fallen hier häufig) und eine durchaus auch als spontan und flexibel wahrgenommene Kultur bei ehemals FFFBeschäftigten.

Abbildung 9: $\quad$ Unternehmenskultur aus Sicht der FFG-MitarbeiterInnen

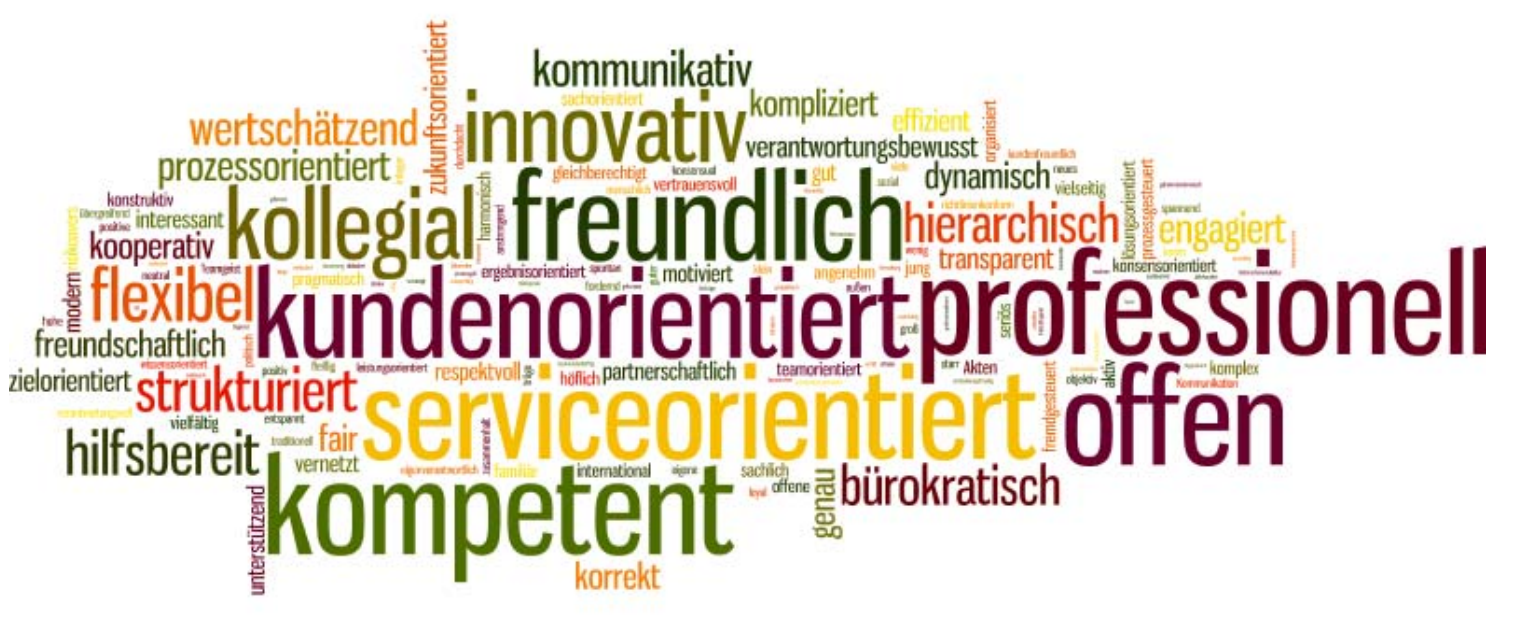

$\mathrm{N}=152$

Anmerkung: N steht für die Zahl der Antwortenden, nicht für die Zahl der genannten Adjektive. Bei den word clouds werden auch Einzelnennungen gezählt. Je häufiger ein und derselbe Begriff genannt wird, desto größer erscheint er. Die Position der Adjektive wird per Algorithmus bestimmt und hat keine tiefer gehende Bedeutung.

Diese beschriebene Selbstwahrnehmung zur Unternehmenskultur mit der starken Betonung von Kunden- und Serviceorientierung steht in Einklang mit den Interviewergebnissen: Dort wurde hervorgehoben, dass der Ausgangspunkt der FFG der Kunde sei, wobei die FFG von ca. 50 Großunternehmen und 2000 kleineren Unternehmen als zentrale Stakeholder ausgeht. Eine Auswahl dieser KundInnen, inklusive einer Auswahl von Forschungseinrichtungen, wurde im Rahmen dieser Evaluierung ebenfalls befragt, wie der folgende Abschnitt ausführt. 


\subsection{Erreichung der Zielgruppen}

Die Fusion der Agenturen war kein Selbstzweck, sondern diente im Endeffekt dem Ziel, die Wirksamkeit der österreichischen Innovations- und Wirtschaftsförderung zu erhöhen, indem hochprofessionelle und effiziente Förderagenturen die Abwicklung der nicht unerheblichen Fördermittel übernehmen. Von daher ist das Gelingen der Strukturreformen schlussendlich nur zu beantworten, wenn die Perspektive der Zielgruppen abgebildet wird. Zunächst betrachten wir jedoch, wie die FFG selbst ihr Verhältnis zu den Zielgruppen einschätzt: Abbildung 10 zeigt, dass die Befragten zu mehr als der Hälfte angeben, dass sie ihre Zielgruppen nunmehr besser erreichen bzw. dass sich auch das Verhältnis zu den Zielgruppen verbessert habe. Negative Einschätzungen sind die klare Ausnahme, es gibt aber auch einen nennenswerten Anteil der Befragten, die keine Veränderung seit der Fusion wahrnehmen.

Abbildung 10: Veränderung der Zielgruppenerreichung seit der Fusion zur FFG

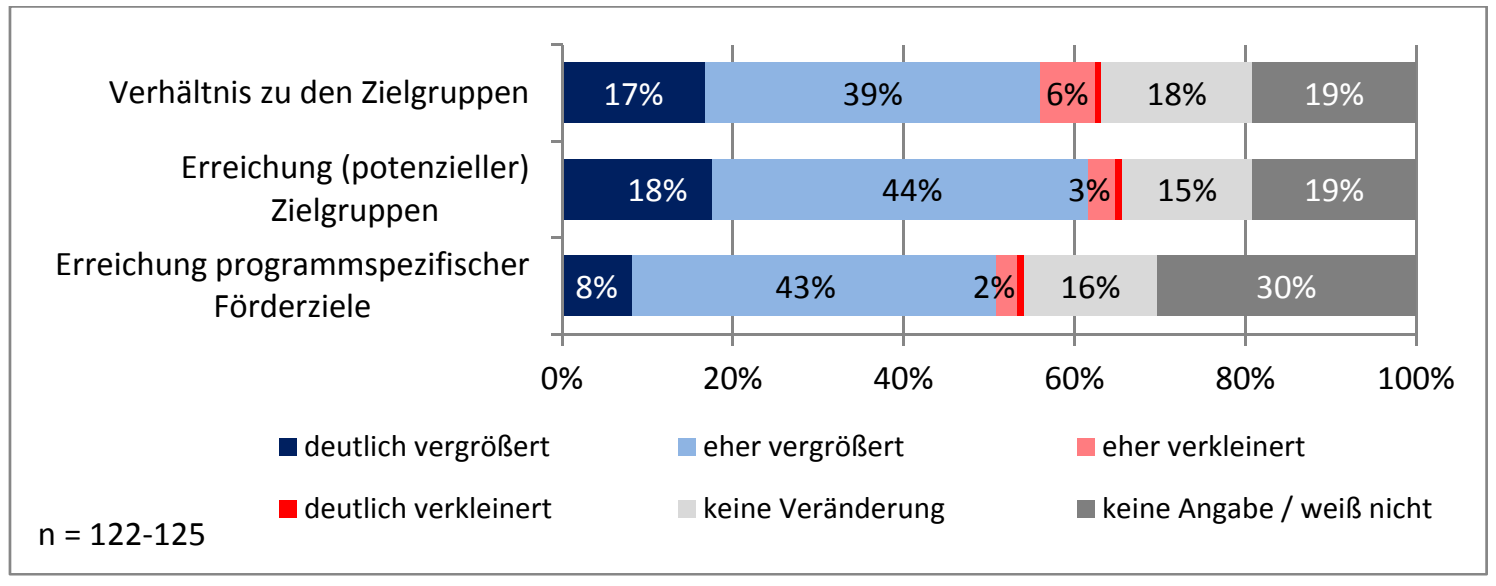

Die FFG hat für die Zielgruppenbefragung aus der Grundgesamtheit aller Antragsteller der Jahre 2014 bis 2016 die Kleinstformate herausgenommen (Innovationsschecks, Praktika etc.). Bei den verbleibenden Adressen wurden jene AnsprechpartnerInnen ausgewählt, die im Betrachtungszeitraum in der betreffenden Organisation für die meisten Projekte verantwortlich waren. Aus dieser Gruppe wurden dann per Zufallsauswahl etwa 600 Antragstellerlnnen ausgewählt, deren Anträge genehmigt wurden und 150 AntragstellerInnen, die auch oder ausschließlich abgelehnte Projekte hatten. Insgesamt wurden 719 Einrichtungen angeschrieben, wovon 260 an der Befragung teilgenommen haben. Dies entspricht einem Rücklauf in Höhe von 36\%. Unter den Befragten, die hierzu eine Angabe gemacht haben, befinden sich 154 Unternehmen und 77 Forschungseinrichtungen. Die Mehrzahl der Befragten hatte in den letzten fünf Jahren mehrheitlich oft (41\%) oder manchmal (39\%) Kontakt zur FFG, 14\% auch selten bzw. 6\% nie. Von den 
Befragten haben 221 eine Förderung erhalten, was mit 90\% aller Angaben die eindeutige Mehrheit darstellt.

Die Zielgruppen der FFG bewerten die Veränderungen seit der Fusion größtenteils positiv: Vor allem die Unterstützung ihrer Anliegen wird als deutlich verbessert wahrgenommen, worin sich das Selbstverständnis der FFG als hauptsächlich am Kundennutzen orientiert widerspiegelt. Ebenfalls sehr geschätzt werden die Klarheit von Zuständigkeiten und die allgemeine Hilfestellung und Kommunikation.

Kritisch hingegen wird der gestiegene administrative Aufwand bewertet, der als deutlich mühsamer als in der Vergangenheit empfunden wird. Auch Unternehmensinterviews haben diesen Punkt bekräftigt, der jedoch nicht nur die FFG betrifft, sondern generell in den Rahmenbedingungen des österreichischen und europäischen Forschungs- und Innovationssystems begründet liegt. Im Vergleich zu anderen Antwortkategorien wird auch das Prozedere der Projektabwicklung kritisch eingeschätzt.

Abbildung 11: $\quad$ Beurteilung der Veränderung durch die FFG-Fusion

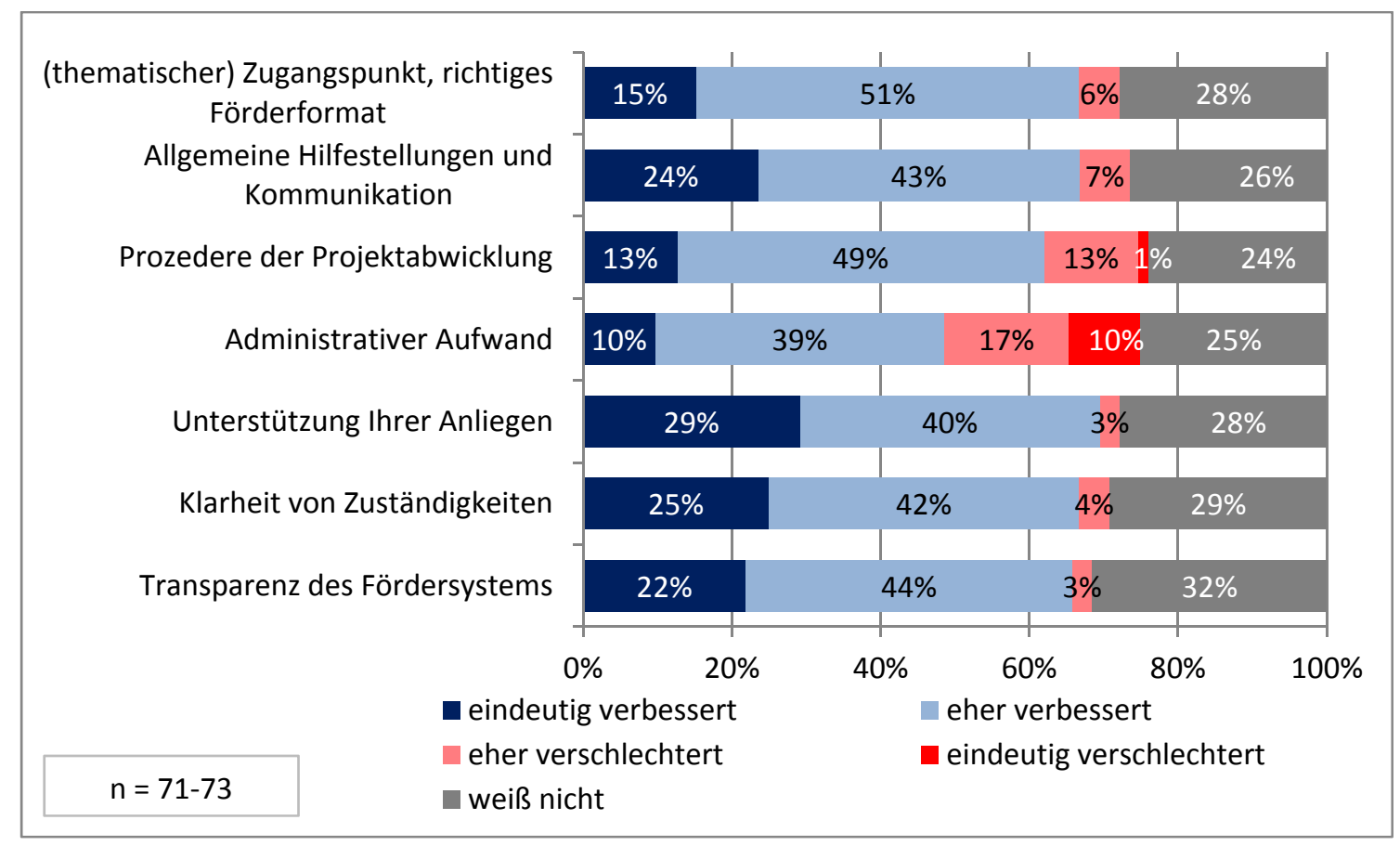

Betrachtet man nun die allgemeine Zufriedenheit der FFG-KundInnen mit ausgewählten Qualitätsdimensionen, so ist zunächst eine ausgezeichnete Bewertung der Professionalität (93\% sehr gut bzw. gut) und Kompetenz der FFG (92\% sehr gut bzw. gut) festzuhalten. Auch die Kundenorientierung (80\% sehr gut bzw. gut) und die Effizienz werden (73\% sehr gut bzw. gut) von der klaren Mehrzahl der Befragten positiv eingeschätzt. 
Abbildung 12: $\quad$ Einschätzung der FFG hinsichtlich ausgewählter Qualitätsmerkmale

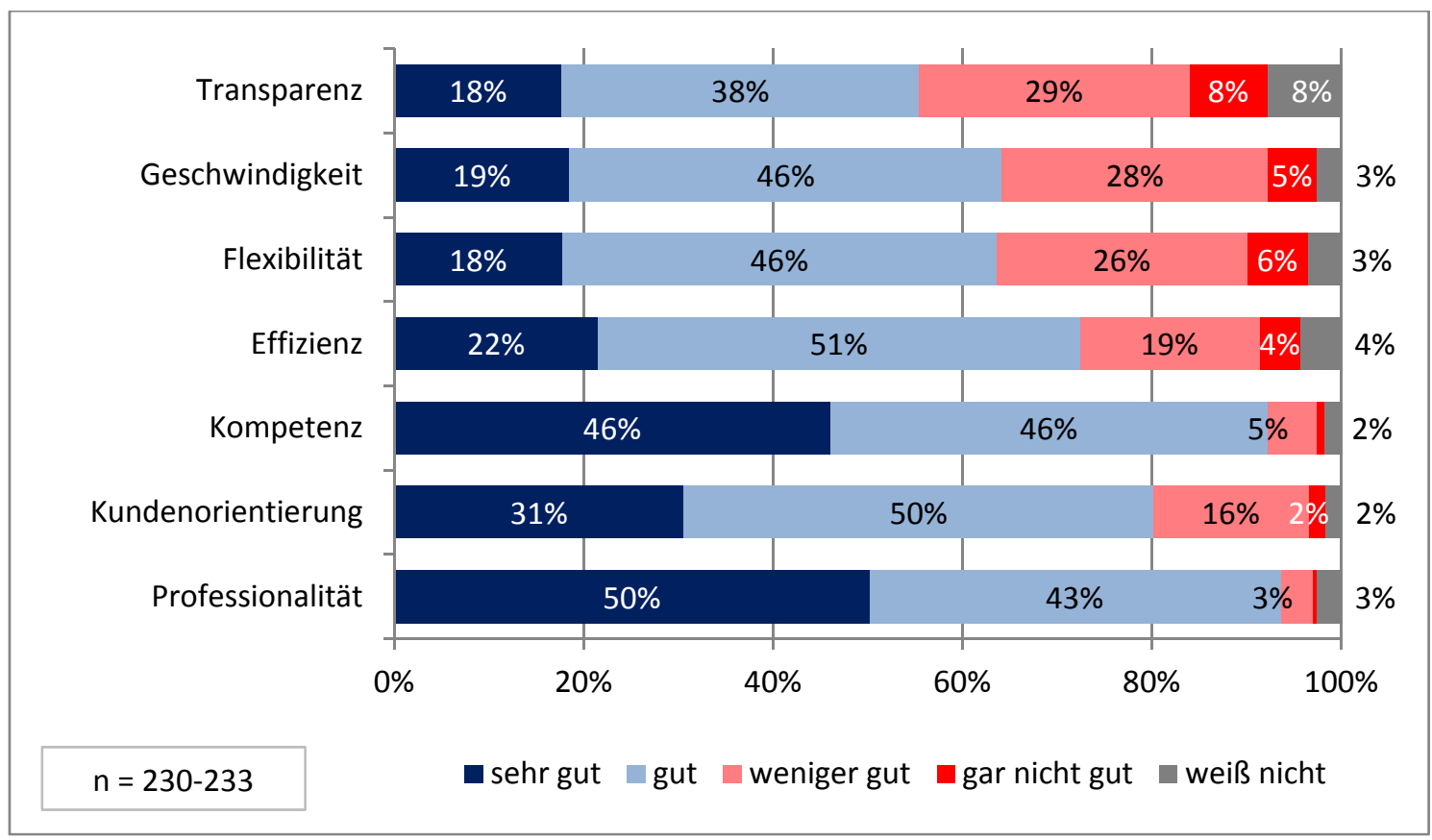

Ein knappes Drittel an Befragten äußert sich jedoch hinsichtlich Transparenz, Flexibilität und Geschwindigkeit der Entscheidungsprozesse weniger zufrieden, woraus sich ein Verbesserungsbedarf ableiten lässt. Dieses Ergebnis steht in einem gewissen Widerspruch zu den Bemühungen der FFG, für den Kern der Geschäftsprozesse ein hohes Maß an Standardisierung vorzugeben, damit die KundInnen Transparenz und Klarheit über die Verfahrensweise gewinnen können und damit die Entscheidungen auch kundenseitig besser eingeschätzt werden können. Wie die Diskussion im Rahmen des Validierungsworkshops ergab, plant man insbesondere bei der Begründung von Ablehnungen in Zukunft (noch) kundenfreundlicher vorzugehen.

Als eine Herausforderung lässt sich konstatieren, dass zahlreiche Fördernehmer vor allem aus dem Unternehmensbereich es für kompliziert halten, die passende Unterstützungsmaßnahme zu identifizieren. Hier haben also auch neu etablierte Instrumente wie der Quick Check und der Förderpilot (vgl. Kapitel 6) noch nicht für ausreichend Klarheit gesorgt, vielmehr spiegelt sich hier mutmaßlich das sehr ausdifferenzierte und stellenweise auch kleinteilige Förderportfolio der FFG wieder. 
Abbildung 13: Wie einfach oder kompliziert ist es aus Ihrer Sicht, die für Ihr Anliegen am besten geeignete Unterstützungsmaßnahme bei der FFG zu finden?

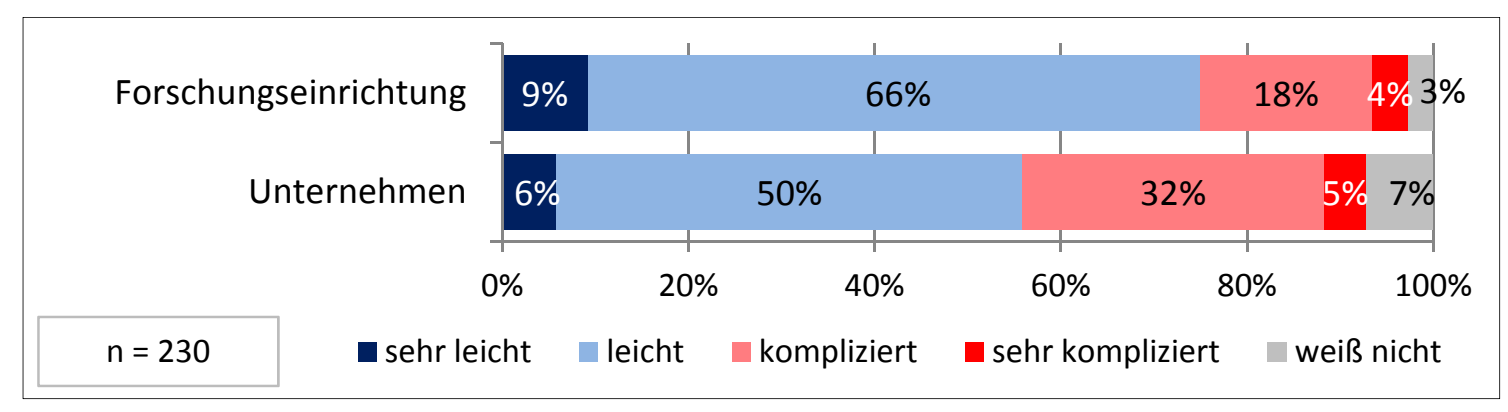

In den Unternehmensinterviews wurde das Förderportfolio in Österreich insgesamt zwar überaus positiv bewertet, da man für jedes Anliegen ein geeignetes Unterstützungsangebot findet. Dass dies systemisch betrachtet aber auch zu negativen Effekten in Form einer gewissen Unübersichtlichkeit und damit einhergehenden Informations- und Suchkosten sowie unterkritischen und damit kaum effizient zu administrierenden Programmen führt, wurde bereits benannt.

\subsection{Fazit}

Für die FFG kann insgesamt festgehalten werden, dass es sich um eine hochprofessionelle und exzellent gemanagte Förderagentur handelt. Die operative Umsetzung von Programmen durch die FFG ist unstrittig herausragend und auch die Entwicklung der Abwicklungskosten zeigt, dass die FFG ihre Aufgaben sehr effektiv und effizient erfüllt. Die Vereinheitlichung der Formate, Spielregeln und Terminologien durch einheitliche Standards auch bei Jury-Prozessen sind für alle Beteiligten von Vorteil.

Zweifellos ist es der FFG durch ihre Größe, aber vor allem durch ihr sehr erfolgreiches Agieren gelungen, sich als der zentrale Akteur für die Förderung von Forschung, Technologie, Entwicklung und Innovation in Österreich zu positionieren. Diese starke Stellung sollte jedoch einhergehen mit einer größeren Sensibilität für die Bedarfe anderer Akteure, beispielsweise was die Öffentlichkeitsarbeit angeht: Von VertreterInnen aus den Ressorts wird zu Recht kritisiert, dass Förderprogramme in der Öffentlichkeit oftmals als "FFG-Programme" wahrgenommen werden, ohne auf den maßgeblichen Beitrag der Ressorts zu diesen Programmen hinzuweisen.

Die FFG verfügt mittlerweile aufgrund der Vielzahl und des Umfangs ihrer Aufgaben über eine exzellente Informations- und Datenbasis zu den Bedürfnissen und den Herausforderungen des österreichischen Forschungs- und Innovationssystems. Schon in der Vergangenheit, kurz nach der Fusion, hat sich die FFG dadurch ausgezeichnet, dass sie 
neue Förderimpulse wie die Stärkung der Zusammenarbeit zwischen Wirtschaft und Wissenschaft aktiv in ihr Portfolio aufgenommen und ausgebaut hat. Dies geschieht nicht zuletzt durch die hervorragende internationale Einbindung beispielsweise in TAFTIE, dem Zusammenschluss europäischer Förderagenturen für die angewandte Forschung.

Die Größe der FFG ist nicht nur national von Vorteil, um österreichweit Themenschwerpunkte gut administrieren zu können, sie ist vor allem auch für die internationale Positionierung faktisch unverzichtbar, damit weiterhin die internationale Anbindung von Unternehmen sowie die Teilnahme von Unternehmen an EU-Projekten so gut gelingt wie in der Vergangenheit.

Es gibt aber auch einige Herausforderungen, denen sich die FFG zu stellen hat. Diese liegen sowohl innerhalb ihres Verantwortungsbereichs als auch außerhalb bei den Eigentümerressorts.

Herausforderungen innerhalb des Einflussbereichs der FFG betreffen zunächst die internen Strukturen und Prozesse, die sowohl zu einer Kultur der Fehlervermeidung als auch zu einer gewissen Unzufriedenheit unter den MitarbeiterInnen hinsichtlich Freiheitsgrade und Einbezug in Entscheidungsprozesse führen. Es kann auch der Eindruck nicht ganz von der Hand gewiesen werden, dass sich manche Bereiche in die Beherrschung der operativen Prozesse gleichsam eingegraben haben und damit den Blick für das große Ganze verlieren. Hier gilt es für die FFG in Zukunft erneut zu justieren, wie viele Prozesse, Kontrollsysteme und standardisierte Verfahren tatsächlich notwendig sind, wie detailliert beispielsweise auch die Instrumenten-Leitfäden ausfallen müssen, und wo Vereinfachungen und mehr Flexibilität implementiert werden können.

Eine zweite Herausforderung betrifft das komplexe Förderportfolio, das einige der FFGKundInnen zu überfordern scheint, trotz Instrumentenkoffer ${ }^{3}$ und Standardisierung der Angebotseinreichung. Außerdem haben einige der Förderangebote eine unterkritische Größe, was zu einem ungünstigen Verhältnis von Aufwand und Nutzen führt. Hier sollte die FFG noch stärker als in der Vergangenheit gegenüber den Eigentümerressorts darauf dringen, schnell zu einer Konsolidierung des Angebots zu gelangen (vgl. Kapitel 9).

Auch wenn sich die Beteiligten gut mit den räumlichen Gegebenheiten arrangiert haben, so hindert die Tatsache, dass nach wie vor räumliche Außenstellen existieren und somit nicht alle MitarbeiterInnen unter einem Dach zusammenarbeiten können, effizientes Ar-

3 Der Instrumentenkoffer ist im Mehrjahresprogramm 2012-2015 dargestellt (FFG 2014, Seite 27). Er wurde gemeinsam mit den Ressorts entwickelt und fasst die Förder- und Finanzierungsinstrumente der FFG in strukturierter und übersichtlicher Weise zusammen. 
beiten, da u.a. der informelle Austausch eingeschränkt wird. Hier wäre der FFG zu wünschen, dass die Eigentümer mehr Planungssicherheit hinsichtlich der personellen Entwicklung herstellen und auf dieser Basis eine Perspektive für einen gemeinsamen Standort für alle FFG-MitarbeiterInnen entwickelt werden kann.

Die zentrale Herausforderung sowohl innerhalb als auch außerhalb der FFG ist die Beeinträchtigung der Arbeiten durch die Vielzahl unterschiedlicher Beauftragungsmodi und Ausführungsverträge. Hinzu kommt eine Komplexitätserhöhung durch die Mittelverwaltung im Auftrag von Stiftungen, die von Auftraggeber-Seite ein durchaus probates Mittel waren bzw. sind, um Budgets für bestimmte Themen zu reservieren. Von Auftragnehmer-Seite (den Agenturen) waren sie bislang insofern geeignet, als man dort innovativere Programmideen lancieren konnte, die von den Ressorts eventuell nicht unterstützt worden wären. Für eine konstantere Finanzierungsbasis von Programmen sind sie jedoch nicht sehr geeignet. Weiters erhöht sich in den Agenturen der administrative Aufwand. Entsprechend sollte dringend eine Simplifizierung der Prozesse, eine Reduktion der Programmvielfalt und eine Optimierung der Schnittstellen zu den Eigentümerressorts angegangen werden. Konkrete Wege dorthin zeigen sowohl die Kapitel zur Governance (Kapitel 7) als auch zu den Zukunftsoptionen und Empfehlungen (Kapitel 9 und 10) auf, wo insbesondere auch die längerfristigen Perspektive eines Globalbudgets thematisiert wird.

Wenn wir nun abschließend reflektieren, was diese Befunde für die Frage der Strukturreformziele bedeuten, so lässt sich folgendes Fazit ziehen: Die FFG hatte sich mit der Fusion im Jahr 2004 insofern einer günstigeren Ausganglage gegenübergesehen, als hier bereits erste Lehren aus der aws-Gründung gezogen worden waren. Aufgrund dessen kann die FFG auf eine durchaus beständige Entwicklung bis zur Gegenwart zurückblicken. Eine der Akzeptanz des Fusionsprozesses förderliche Entscheidung der Geschäftsführung war, die Vorläuferorganisationen im Kern als solche zu belassen und kein vollständiges Aufgehen in neuen Strukturen anzustreben. Dadurch sind die Vorläuferorganisationen auch heute noch in der Organisationsstruktur sichtbar und teilweise haben auch ältere Unternehmenskulturen noch Bestand. Aufgrund der hohen Funktionalität der aktuellen Organisation besteht aber kein Anlass, daran etwas zu ändern. Insgesamt lässt sich konstatieren, dass die FFG nach einem erfolgreich abgeschlossenen Fusionsprozess nunmehr als eine Organisation nach innen funktioniert und nach außen auftritt, die gemeinsame Werte, Normen und Rollenverständnisse vertritt. Dieser Befund wird auch dadurch unterstützt, dass unter den MitarbeiterInnen eine hohe Identifikation und Zufriedenheit mit ihrer Agentur vorliegt. 
Die FFG ist im Laufe der Jahre personell und vom verantworteten Budget her kontinuierlich gewachsen, die Abwicklungskosten selbst haben sich in Relation zu den operativen Mitteln jedoch verringert, was die hohe Effizienz und Effektivität der FFG unterstreicht.

Neben zahlreichen veränderten Rahmenbedingungen wie der Einführung der wirkungsorientierten Haushaltsführung, der gestiegenen Bedeutung des Rechnungshofes, zahlreicher neuer Themen und Instrumente in der Forschungs- und Innovationsförderung wie auch insgesamt einer zunehmenden Komplexität in der (österreichischen) FTI-Politik hat insbesondere die Einführung gemeinsamer interner Prozesse, Standards und interner Kontrollsysteme im Jahr 2010 das Handeln der FFG als Organisation geprägt. Diese sorgen einerseits für die allseits akzeptierte hohe Professionalität der FFG, lassen andererseits aber wenig Interpretationsspielraum für die MitarbeiterInnen.

Die Fusion und in Folge die Positionierung der FFG als der zentrale Ansprechpartner in Österreich für die Förderung von Forschung, Technologie, Entwicklung und Innovation hat zu einer deutlichen Zunahme der Handlungsspielräume geführt, sowohl hinsichtlich Programmabwicklung (wie intendiert) als auch Programmdesign: Zwar bleibt den Eigentümerressorts die Letztentscheidung darüber, welche Programme in welcher Form aufgelegt werden, dies erfolgt aber oftmals in enger Zusammenarbeit mit FFGVerantwortlichen, die aufgrund ihrer exzellenten Datenbasis und FTI-Expertise hierzu wichtiges strategisches und operatives Wissen beisteuern können.

Bleibt zum Schluss die Frage nach der Zielgruppenerreichung: Diese ist insgesamt positiv zu werten, ist es der FFG doch unter anderem in den vergangenen Jahren gelungen, auch zahlreiche neue KundInnen aus dem Unternehmensbereich zu adressieren. Generell sind die Zielgruppen mit der aktuellen Situation auch sehr zufrieden mit der FFG und bewerten vor allem die seit der Fusion eingetretenen Veränderungen wie Transparenz des Fördersystems, Klarheit von Zuständigkeiten, Unterstützung ihrer Anliegen und allgemeine Hilfestellung und Kommunikation sehr positiv. Es bleibt jedoch die ernst zu nehmende Kritik am administrativen Aufwand für die Fördernehmer und an der Kompliziertheit, das richtige Förderformat zu finden als Aufruf an alle Beteiligten, d.h. die Agenturen sowie die Eigentümerressorts, hier in Zukunft für mehr Vereinfachungen zu sorgen. 


\section{$5 \quad$ Die Austria Wirtschaftsservice Gesellschaft aws}

Die aws (Austria Wirtschaftsservice Gesellschaft $\mathrm{mbH}$ ) unterstützt österreichische Unternehmen dabei, Ideen umzusetzen und neue Produkte zu entwickeln und bis zum Markterfolg zu führen. Der aws steht hierbei eine breite Palette an Instrumenten zur Verfügung: Kredite, Zuschüsse, Garantien, Eigenkapital und Coaching. Die aws nimmt unter den Förderungsagenturen insofern eine Sonderstellung ein, als sie in erster Linie die Aufgaben einer Förder- und Finanzierungsbank übernimmt. Allerdings hat die aws ihre Konzession zum Betrieb von Bankgeschäften zum Ende des Geschäftsjahres 2013 zurückgegeben, d.h. sie betreibt keine Geschäfte zur Refinanzierung am Kapitalmarkt. Sie ist eine $\mathrm{GmbH}$, die zur Gänze in der Eigentümerschaft des Bundes steht. Im Jahr 2015 betrug das Förder- und Finanzierungsvolumen rund 826 Mio. Euro. Die aws konzentriert sich auf drei Kernfunktionen:

- Förderungen für Gründer und junge Unternehmen.

- Förderungen für wachsende Unternehmen insbesondere auch in Regionalförderungsgebieten zur Sicherung des Standorts und der Wettbewerbsfähigkeit sowie zur Schaffung und Erhaltung von Arbeitsplätzen.

- Spezialist für Technologieverwertung und Innovationsförderung, speziell in den Hochtechnologiebereichen Informations- und Kommunikationswissenschaften, Life Sciences (Life Science Austria - LISA), Physical Sciences und der Kreativindustrie (impulse).

Laut aws-Gesetz $\S 2$ ist ihre Aufgabe die "Vergabe und die Abwicklung von unternehmensbezogenen Wirtschaftsförderungen des Bundes sowie die Erbringung sonstiger, im öffentlichen Interesse liegender Finanzierungs- und Beratungsleistungen zur Unterstützung der Wirtschaft". Dies beinhaltet die Abwicklung der unternehmensbezogenen Wirtschaftsförderungen (Begleitung von der Gründung bis zum Börsengang als so genannter "one-stop-shop") und die Erbringung sonstiger, im öffentlichen Interesse liegender Finanzierungs- und Beratungsleistungen zur Unterstützung der Wirtschaft.

Der Fokus der aws-Tätigkeit liegt bei Unternehmen, in deutlich geringerem Ausmaß sind Hochschulen, Forschungseinrichtungen und EinzelforscherInnen Zielgruppe von Förderungsaktivitäten. Die Unternehmen werden durch ExpertInnen der aws zu ihren Problemstellungen beraten und die aws stellt den passenden Instrumentenmix für den konkreten Fall zusammen.

Gemäß ihrer Funktion als Förderbank haben Garantien sowie zinsgünstige (ERP-) Kredite/Darlehen für die aws im Vergleich zu den direkten Zuschüssen und Beratungen (damit sind nicht Beratungen im Zusammenhang mit einer Förderung gemeint, sondern spe- 
zifische Beratungen wie z.B. zu IPR-Fragen) eine deutlich höhere Bedeutung. Für Eigenkapitalmittel stehen der aws unter anderem der Mittelstandsfonds, die aws VentureCapital-Initiative, der aws Gründerfonds und der aws Business Angel Fonds zur Verfügung. Darüber hinaus setzt die aws beispielsweise in den Bereichen Gründungen, Kreativwirtschaft und Entrepreneurship durch Zuschussprogramme Impulse.

Die Ministerien bmwfw und bmvit vertreten den Eigentümer Bund gegenüber der aws, für den ERP Fonds ist dies nur das bmwfw. Die Schadloshaltung der Garantien, die durch die aws übernommen werden, liegt beim Bundesministerium für Finanzen (BMF), weshalb das BMF ein weiterer wichtiger Akteur der Governance-Struktur ist (siehe dazu auch Kapitel zu den finanziellen Risiken).

\subsection{Historie und Vorläuferorganisationen}

Entstanden ist die aws 2002 durch die Verschmelzung der Finanzierungsgarantie-Gesellschaft FGG, der Innovationsagentur und der BÜRGES Förderbank. Seit ihrer Gründung übernimmt die aws auch die Geschäfte des ERP-Fonds (Mittel, die die Republik Österreich aus dem Titel des Marshall-Plans nach dem Zweiten Weltkrieg erhielt) sowie die Geschäftsführung der Nationalstiftung (dotiert aus Mitteln des Bundes, der Österreichischen Nationalbank und des ERP-Fonds).

Die aws entwirft Mehrjahresprogramme, die - nach Empfehlung des Aufsichtsrates - von bmwfw und bmvit genehmigt werden müssen und die Indikatoren zur Messung der Zielerreichung, einen Evaluierungsplan sowie eine indikative Finanzplanung beinhalten.

Die Zusammenführung der aws war geprägt durch finanzielle Schwierigkeiten der FGG, die der Gründung vorausgingen. Bei der aws gab es große Erwartungen hinsichtlich Einsparungspotenziale - es ging nur nachrangig um die anderen Ziele der Strukturreformen. Die erwarteten Einsparungspotenziale bzw. Effizienzziele haben aus Sicht der aws viel Aufmerksamkeit und Ressourcen erfordert, v.a. die Errichtung eines einheitlichen ITSystems. Eine gewisse Einsparung konnte erreicht werden, aber die in den Raum gestellten Vorgaben (20\%) haben sich als unrealistisch herausgestellt, angesichts der Ausweitung des Fördergeschäfts bis 2009 und des dadurch bedingten Wachstums der Or- 
ganisation. Seitdem ist die Beschäftigtenzahl leicht gesunken (siehe Personalstrukturen). Die Einsparungsthematik hat aufgrund der Umsatzsteuerproblematik ${ }^{4}$ seit 2015 wieder starke Relevanz bekommen; Einschnitte mussten rasch umgesetzt werden.

Das Portfolio der aws, das zum großen Teil seine Historie in den Förderschwerpunkten der Vorgängerorganisationen hat, aber auch teilweise in den vergangenen Jahren weiter ergänzt wurde (zum Beispiel kam die Förderung der Kreativwirtschaft hinzu), ist heterogen. Es reicht von der Übernahme von großen Garantien für Investitionsvorhaben (z.B. für Umweltschutzmaßnahmen, Energieeffizienzmaßnahmen oder auch Forschungsund Entwicklungs- bzw. Innovationsvorhaben) bis hin zur Abwicklung von Wettbewerben wie "Jugend innovativ". Trotzdem wird der aws von ExpertInnen sowie in vielen Gesprächen mit Verantwortlichen bestätigt, dass die aws klar in der Funktion der Unternehmensförderung und -finanzierung gesehen wird und im Rahmen der österreichischen Wirtschaftsförderlandschaft eine distinkte Position innehat. In den Gesprächen mit Bereichs- und AbteilungsleiterInnen wurde zudem deutlich, dass erst das breite nach der Fusion erreichte Portfolio es ermöglicht, maßgeschneiderte Förder- und Finanzierungspakete für die Kundlnnen zu schnüren. Dabei wird zunehmend wichtiger, dass die gewährte Finanzierung wichtige strukturverändernde Impulse im Unternehmen setzen kann und die Innovationsleistung des Unternehmens gestärkt wird. Hierzu wurde im Mehrjahresprogramm 2014-2016 das Konzept des volkswirtschaftlichen Nutzens entwickelt, das für die Erfolgsmessung aller geförderten Vorhaben und auch für die Projektauswahl eingesetzt wird.

Was nach Ansicht vieler InterviewpartnerInnen eine noch andauernde Aufgabe ist, ist die Erreichung der Zielgruppen. Die Bekanntheit der Marke aws im Vergleich zum Beispiel zur früheren BÜRGES ist noch nicht so hoch. Seit einigen Jahren arbeitet die aws bereits intensiv daran, ihre Bekanntheit in der Zielgruppe weiter zu vergrößern und hat neben einer neuen corporate identity auch ein Kunden-Beratungscenter eingerichtet und im vergangenen Jahr die Website komplett neu als Antwort auf Kundenbedürfnisse gestaltet und die Förderberatung weiter ausgebaut (z.B. den Förderpiloten gemeinsam mit anderen Förderungsgesellschaften).

4 Nach einem im April 2015 ergangenen Urteil des Bundesgerichtshofs ist die aws umsatzsteuerpflichtig. Dadurch entstanden Nachforderungen und zusätzliche Kosten in der Gegenwart, die mit drastischen Einsparungen in der aws einhergehen, sich aber auch auf die verfügbare Fördersumme negativ auswirken. Nach Einschätzung von ExpertInnen wäre das Problem mit einer Basisvergütung für die aws lösbar, weil die Steuern im Augenblick für Leistungen bezahlt werden, die durch die Vollkostenrechnung entstehen. 


\section{Bewertung der Fusion}

Mittlerweile kann konstatiert werden, dass der Fusionsprozess trotz schlechter Startbedingungen erfolgreich abgeschlossen ist und die aws nunmehr als eine Organisation agiert. Neben dem starken Fokus auf Einsparungen, der viele Jahre lang prägend war, war in den ersten Jahren die Geschäftsführung der aws nicht optimal besetzt, weswegen die aws in dieser Zeit nicht zu ihrer vollen Leistungsfähigkeit kam. Die interne Organisationsentwicklung schritt, getrieben von der mittleren Führungsebene, dennoch weiter erfolgreich voran. Als wesentliche Ereignisse in der Organisationsentwicklung sind die folgenden anzusehen: (1) der Umzug in die Walcherstraße (2013), (2) die Etablierung einheitlicher Systeme, zunächst für Gehalt (2005), später auch für die IT (2010), (3) der Leitbildprozess (2015, sowie erstmalig 2009) sowie (4) Teambuilding-Maßnahmen und Management-Klausuren (seit 2009).

\subsection{Interne Strukturen und Prozesse}

Die aws agiert dank ihrer Effizienz- und Prozessorientierung hoch professionell. Seit der Fusion wurden viele Ressourcen in die Organisationsentwicklung gesteckt und eine Vielzahl moderner Management-Instrumente zu ihrer Steuerung implementiert. Dadurch konnten Prozesse optimiert und verkürzt werden.

\subsubsection{Aufbauorganisation}

Die Aufbauorganisation der aws hat sich seit der Fusion mehrmals verändert und stellt eine Struktur dar, die auf die Auftraggeber (und Eigentümer) ausgerichtet ist. Damit sind die Programme nach Förderungsschwerpunkten gegliedert und gleichzeitig auch die Vorläuferorganisationen noch erkennbar. Sie sind in drei große Förderbereiche gegliedert: Kredite und Kofinanzierungen (hier werden u.a. die Geschäfte des ERP-Fonds oder die Nutzung europäischer Fonds u.a. EFRE und ELER abgewickelt), Garantien und Eigenkapital (worin das Garantiegeschäft der FGG aufgegangen ist sowie viele KMUFörderaktivitäten der BÜRGES-Bank) und Entrepreneurship, Schutzrechte und Seedförderungen (u.a. inklusive der Tätigkeiten der Innovationsagentur). 
Abbildung 14: $\quad$ Organigramm aws

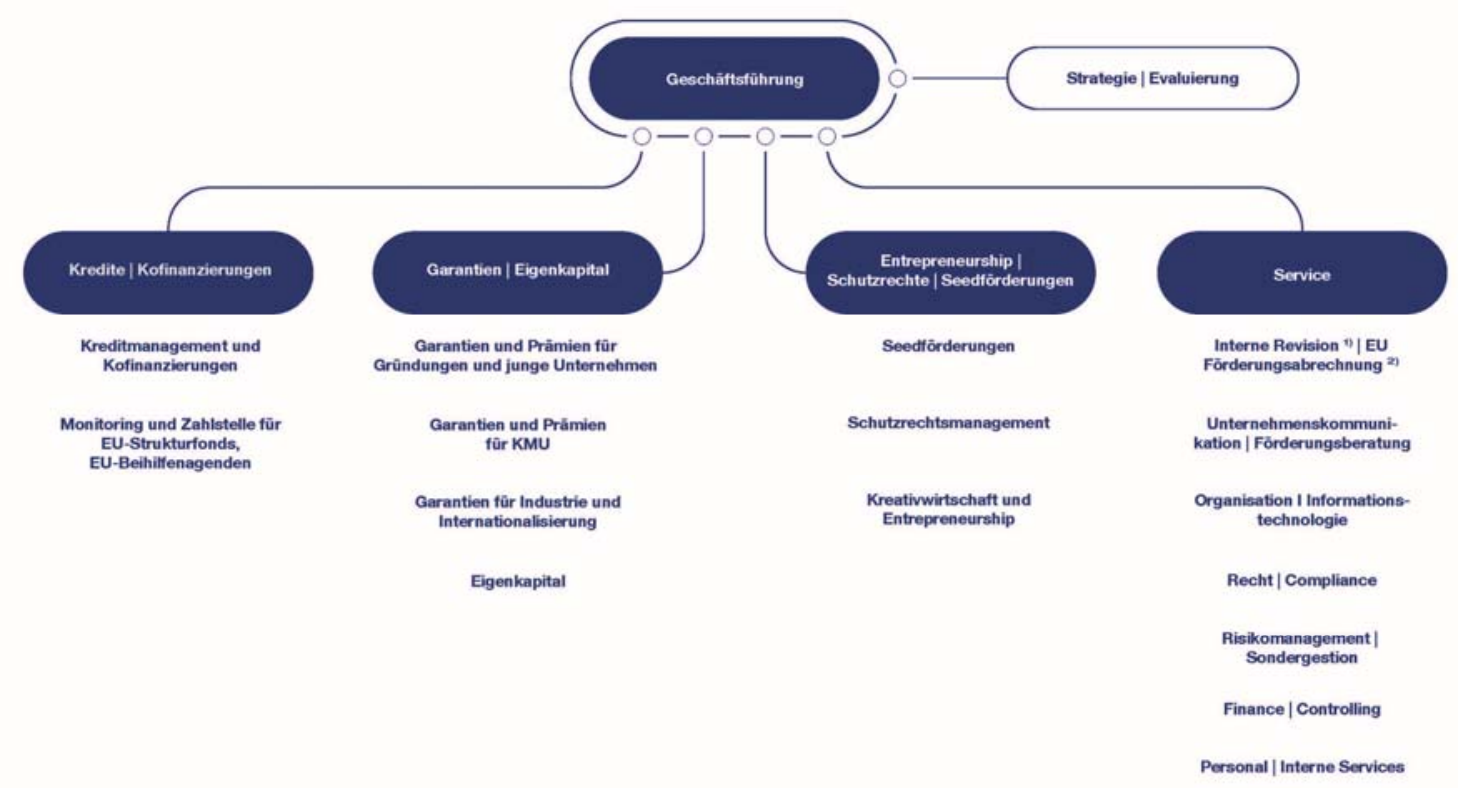

Der ERP-Fonds ist ein eigenes Rechtskonstrukt (nach dem ERP-Fonds-Gesetz), das eine eigene Berichtslegung und ein eigenes Aufsichtsgremium erfordert. Dadurch, dass alle zwei Jahre ein Nationalratsbericht zu erstellen ist und die im Nationalrat vertretenen Parteien anteilsmäßig Mitglieder der ERP-Kreditkommission benennen dürfen, ist der ERP-Fonds stärker politisch kontrolliert.

Die internen Service-Abteilungen erfüllen die Querschnittsaufgaben wie Personal, IT, Controlling, Unternehmenskommunikation sowie die förder- bzw. finanzdienstleistungsspezifischen Aspekte Recht und Risikomanagement. In innen ist das Servicepersonal der vier Vorgängerorganisationen aufgegangen, und aufgrund von Einsparungen ist der Bereich über die Jahre insgesamt etwas kleiner geworden (siehe Personalstrukturen). Durch intensive Personalentwicklung haben sich die Service-Abteilungen nach der Fusion sehr stark professionalisieren und die notwendigen Prozesse der Standardisierung und Vereinheitlichung wesentlich unterstützen können. Vergleichbare Agenturen haben manche Services nach außen vergeben, wie z.B. Travel Management. Die aws hält ihre interne Lösung dagegen für kostengünstiger.

Im Rahmen ihrer Eigenkapitalförderung hat die aws vielfältige Unternehmensbeteiligungen aufgebaut, die über fünf Tochterunternehmen abgewickelt werden, deren Geschäfte aber im Konzernabschluss bilanziert werden. Außerdem ist bei der aws die Geschäftsstelle für die Nationalstiftung angesiedelt, die deren operative Abwicklung übernimmt.

Auch für die aws gilt, dass hier eine Vielzahl unterschiedlicher Abteilungen und Vorläuferorganisationen existieren, so dass es intern durchaus unterschiedliche Kulturen in den 
einzelnen Bereichen gibt, was jedoch nicht kritisch zu sehen ist. In ihren Ablaufprozessen gelingt die interne Koordination, so dass die aws den Kundlnnen gegenüber in der Regel einheitlich und durch einen Ansprechpartner repräsentiert ("one face to the customer") auftritt (siehe auch dazu das Kapitel Ablauforganisation). Insgesamt tragen die internen Prozesse sehr gut dazu bei, dass die aws nach außen einheitlich auftreten kann (z.B. die elektronische Abwicklung der Antragstellung und Förderung).

\subsubsection{Beauftragungssystem}

Während im Fall der FFG das FFG-Gesetz und das Forschungs- und Technologieförderungsgesetz (FTFG) die wesentlichen gesetzlichen Grundlagen für Beauftragungen darstellen und Rahmenbedingungen abgrenzen, ist das im Fall der aws vielschichtiger. Wesentliche gesetzliche Grundlagen für die aws sind das KMU-Förderungsgesetz, das Garantiegesetz sowie das ERP-Fonds-Gesetz. Zudem sind die beihilferechtlichen Rahmenbedingungen vielschichtiger, da nicht nur FuE-Beihilfen gewährt werden, sondern beispielsweise auch KMU-Förderung oder Regionalförderung.

Diese Vielschichtigkeit bringt es mit sich, dass das Beauftragungssetting auch vielfältig ist. Für die ERP-Kredite ist die aws grundsätzlich mit der Abwicklung beauftragt, hier ist für die Umsetzung erforderlich, dass die aws ein Jahresprogramm entwickelt, das dann von der Bundesregierung beschlossen wird. Im Falle der Garantien, der Beihilfenberatung des Bundes und der Zahlstelle für die europäischen Strukturfonds-Gelder bestehen langjährige Verträge. Viele Beauftragungen laufen jedoch kurzfristig, auf ein Jahr begrenzt, was Planungsunsicherheit mit sich bringt - nicht nur für die Agentur, sondern auch für die KundInnen. Eine strategische Impulssetzung in die Zielgruppe hinein ist dadurch deutlich erschwert.

Aufgrund eines fehlenden Basis-Budgets muss die aws jede Beauftragung auf Vollkostenbasis kalkulieren. Damit ist eine permanente Überwachung der Abwicklungskosten nötig, um abschätzen zu können, ob bis zum Jahresende die nötigen Betriebskosten durch die zu erwartenden Aufträge gedeckt sein werden. Das führt zu unnötiger Budgetunsicherheit, die noch dadurch erhöht wird, dass Freigaben für Aufträge oftmals sehr verzögert vom Ministerium kommen (in extremen Fällen erst im November für das laufende Jahr). Dies stellt eine große Hürde dar, vor allem, wenn es darum geht, Personal für neue Aufträge vorzuhalten. Auch für die Ressorts stellt das Vollkosten-System Nachteile dar, da die veranschlagten Overhead-Kosten als nicht transparent wahrgenommen werden und die Entwicklung der Overhead-Kosten regelmäßig im Rahmen der aws Eigentümer Jour Fixe den Eigentümerressorts berichtet werden müssen. Durch eine Teil- 
kostenrechnung würden gegenüber dem Bund die Höhe der Overheadkosten transparent und könnten vom Bund gezielt beeinflusst werden (z.B. über 20\%-Vorgabe in Eigentümer-Strategie).

Prinzipiell initiiert der Bund neue Programme, aber auch aus der aws heraus werden manchmal Impulse für Programme gesetzt. Diese werden in der Regel bei der Nationalstiftung zur Finanzierung eingereicht und laufen dann 2-3 Jahre, z.B. aws First.

In dieser heterogenen Beauftragungslage muss jedes Programmbudget separat verwaltetet werden, die aws hat keinen Spielraum, der ihr erlaubt, Budgets zwischen Programmen hin- und herzuschieben. Das geht bis zu einem gewissen Grad nur bei den ERPFondsmitteln: hier kann das Budget innerhalb der im Jahresprogramm definierten Themenfelder verschoben werden.

\subsubsection{Personal- und Kostenstrukturen}

Die Entwicklung der Finanzkennziffern für die aws ist in Tabelle 4 dargestellt. Negative Werte beim EGT resultieren v.a. aus den plangemäßen negativen Ergebnissen der Tochtergesellschaften. Diese investieren primär in offene Beteiligungen, deren programmgemäße - mit den Auftraggebern abgestimmten - Verluste sich über den Zeitverlauf aufbauen und die geplanten Gewinne erst im Zuge der Beteiligungsveräußerungen (Exits) realisiert werden,

Das Betriebsergebnis ist daher demgegenüber wesentlich stabiler als das EGT. Die negativen Ergebnisse in den Jahren 2015 und 2016 lassen sich mit den Sondereffekten der Abwicklung der Venture Capital Initiative mit der Nationalstiftung erklären. Diese Sondereffekte ergeben sich aus dem Ergebnisabführungsvertrag zwischen der Venture Capital Initiative und der aws, der sich in verschiedenen G\&V Positionen abbildet, letztlich für die Kostenbetrachtung der aws aber ein Nullsummenspiel ist. Die Wirkungsweise des Ergebnisabführungsvertrages wurde mit dem aws Aufsichtsrat im Detail diskutiert und von diesem als kostenneutral akzeptiert.

Beim ERP-Fonds Betriebsergebnis gilt ab 2014 eine Gliederung gemäß § 224 bzw. § 231 UGB (betrifft v.a. Zinsen u. ähnliche Erträge bzw. Zinsen und ähnliche Aufwände, die nicht mehr im Betriebsergebnis dargestellt werden). Der Rückgang beim EGT des ERP-Fonds erfolgte v.a. durch niedrigere Zinserträge. 
Die aws-Geschäftsführung hat sich seit dem Jahr 2010 gezielt mit der Steigerung der Produktivität beschäftigt und Maßnahmen implementiert, die deutlich Wirkung zeigen. Als Beispiel dieses langfristigen Veränderungsmanagements wurde beispielsweise auf das Halbieren der Time to Contract (aws-Zeit) seit 2010 hingewiesen. Darauf aufbauend wurde seit 2013 ein Modell zur Messung der Produktivität, das Effizienzentwicklungen eindeutig widerspiegelt, eingeführt. Dabei wurde hier wie folgt vorgegangen: Im Jahr 2013 wurde mit der modellbasierten Messung der Produktivität begonnen, indem das Verhältnis Zusagezahlen/VZÄ gemessen wurde. Dieses Modell ergab für das Jahr 2013 eine Produktivitätssteigerung von 6\%. 2014 wurde die Produktivitätsberechnung dahingehend verfeinert, dass zusätzlich zur Messung Zusagen/VZÄ auch die Bestandbetreuung bzw. die Ablehnungen im Modell der Produktivitätsberechnung Berücksichtigung fanden. Damit wurde sichergestellt, dass alle Aufwand verursachenden Leistungen in die Beurteilung der Effizienzentwicklung aufgenommen wurden. Methodisch kann damit der Aufwand je Förderung über den ganzen Lebenszyklus in Personentagen abgebildet werden (Zyklus: Antrag-Vertrag-Beendigung). Für das Jahr 2014 konnte anhand des verfeinerten Modells eine Produktivitätssteigerung von 3\% nachgewiesen werden. Für 2015 ergab sich anhand dieser Berechnungslogik eine Produktivitätssteigerung von 7,6\%, im Jahr 2016 eine Steigerung von 3,5\%.

Die personelle Ausstattung der aws (gemessen in Vollzeitäquivalenten inklusive Leihkräften) zeigt, dass insbesondere der Service-Bereich kontinuierlich sinkende Personalzahlen aufweist, da man hier das übernommene Personal aus den Alt-Gesellschaften über die Jahre nicht ergänzt hat, sondern Abgänge zur Kosteneinsparung genutzt hat.(vgl. Abbildung 15). Nur in wenigen Fällen werden zusätzliche SpezialistInnen eingestellt (z.B. im IT- oder Personalbereich).

Abbildung 15: Personelle Ausstattung der aws* (aktive MitarbeiterInnen in VZÄ zum Stichtag 31.12., inklusive Leihpersonal)

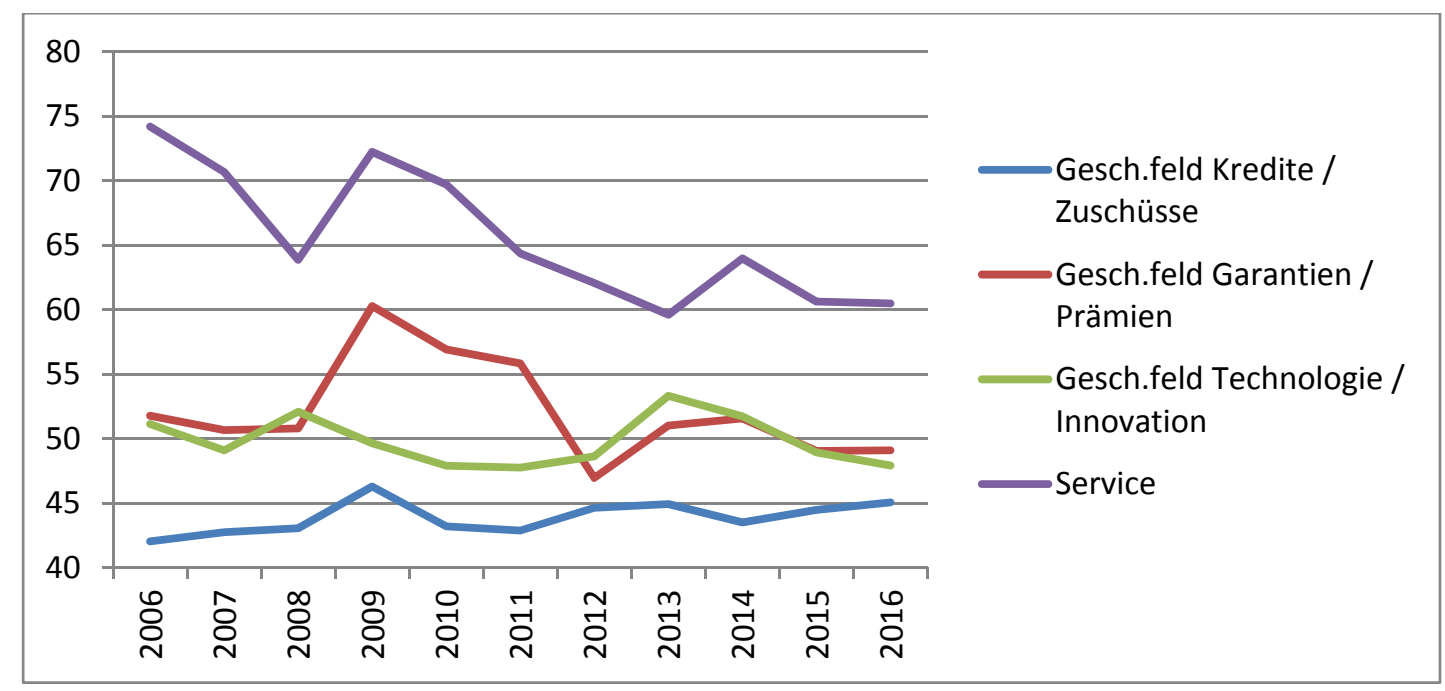

*Aus Gründen der Übersichtlichkeit wurde das für die interne Revision zur Verfügung stehende Personal hier nicht abgebildet, die Anzahl der VZÄ für diesen Bereich betrug jedoch maximal 2,6 VZÄ. 
Insgesamt sind nach der Fusion die Personalzahlen leicht rückläufig gewesen, da Abgänge nicht immer ersetzt wurden. Mit zunehmendem Auftragsvolumen stieg die Anzahl der MitarbeiterInnen in den Geschäftsfeldern deutlich an und war 2009 auf dem Höchststand. Die in Folge der Finanzkrise durchgeführten Einsparungen der Regierung betrafen auch das Fördergeschäft, so dass Personal sozialverträglich abgebaut wurde.

Personalentwicklung wird als Instrument von den Führungskräften sehr ernst genommen. Sie konzentriert sich vorwiegend auf die Förderung von Fachexpertise, in etwas geringerem Umfang auf Führungsaufgaben. In der flachen Hierarchie der Agentur mit jeweils größeren fachlichen Abteilungen sind Aufstiege in der Hierarchie weniger möglich. Innerhalb des Hauses wird Job-Rotation gefördert, damit die MitarbeiterInnen viel Einblick in verschiedene Bereiche erhalten.

Die regelmäßig durchgeführten MitarbeiterInnenbefragungen der aws weisen in den letzten Jahren zunehmend eine hohe Mitarbeiterzufriedenheit aus. Auch in der vom Fraunhofer ISI im Rahmen der gegenständlichen Evaluierung durchgeführten Befragung finden sich hohe Zufriedenheitswerte, aber an der einen oder anderen Stelle durchaus auch Kritik, wie in den folgenden Abschnitten näher ausgeführt wird.

Basierend auf aktuellen Personaldaten (Stand: Herbst 2016) hat das Evaluationsteam eine Gesamtbefragung aller MitarbeiterInnen durchgeführt. Insgesamt wurden 243 Personen angeschrieben. An der Befragung teilgenommen haben 154 Personen, definiert als diejenigen, die mindestens die Hälfte der Fragen beantwortet haben. Dies entspricht einer Rücklaufquote von $63 \%$.

Für die Interpretation der Ergebnisse gilt es zu berücksichtigen, dass v.a. Mitarbeiterlnnen aus dem Bereich Garantien geantwortet haben, gefolgt von Krediten und Seedförderungen. Doch auch der Bereich der internen Services ist gut vertreten. Von den Aufgabenschwerpunkten her stammt bei der aws mehr als die Hälfte der Befragten aus dem Bereich Projektmanagement, die demnach die folgenden Antworten auf die im Rahmen der MitarbeiterInnen-Befragung gestellten Fragen dominieren.

Basierend auf unserer Ausgangshypothese, dass die Beschäftigung in einer der vier Vorläuferorganisationen nach wie vor die normativen Werte und Überzeugungen prägt, haben wir in der Befragung auch erhoben, ob und wenn ja, in welcher der Vorläuferorganisationen die Befragten beschäftigt waren. Dies ist für exakt die Hälfte der Befragungsteilnehmerlnnen der Fall. Viele kommen aus der BÜRGES oder dem ERP-Fonds, aber auch aus der FGG. In der Innovationsagentur früher beschäftigt war nur ein kleinerer Teil. 


\subsubsection{Ablauforganisation}

Die aws verfügt über ein Prozesstool mit definierten Standards. Die Ablauforganisation ist untergliedert in Management-, Geschäfts- und Customer-Relation-Prozesse. Außerdem gibt es Service-Prozesse.

Die internen Prozesse der aws sind darauf ausgerichtet, den Kundlnnen schnell ein passendes Lösungspaket zu erarbeiten. Die Koordinationsprozesse zwischen den Förderabteilungen funktionieren einwandfrei, ebenso die Zusammenarbeit mit den internen Services. Interne Instrumente wie das Rating zur Bewertung finanzieller Risiken, die Bewertung des Projekterfolgs (Volkswirtschaftlicher Nutzen) oder die IT-gestützte Förderabwicklung sind vereinheitlicht, sie sind aber so aufgebaut, dass sie für die verschiedenen Instrumente der aws passfähig sind. Die InterviewpartnerInnen wurden gebeten, zu reflektieren, ob das Verhältnis zwischen Zentralisierung bzw. Einheitlichkeit und individuellen Bewertungsspielräumen ausgewogen sei. Dies wurde durchgehend bestätigt.

Die zahlenorientierte interne Steuerung der aws ist ein Ausdruck der erreichten Professionalität, allerdings weisen einige Interviewaussagen auf eine zu starke Zahlenorientierung bzw. ein Zuviel an Management-Tools hin. Die Geschäftsführung der aws hat darauf hingewiesen, dass die hohe Effizienz der Organisation anders nicht zu erreichen gewesen wäre und ist daher von den Vorteilen dieses Vorgehens überzeugt.

Betrachtet man die Befragungsergebnisse für die aws, dann zeigen sich die Befragten vor allem mit der Art, wie die zentralen Leistungserwartungen kommuniziert werden, der Delegation von Verantwortung und dem Einbezug in Entscheidungsprozesse sehr zufrieden. Weniger zufrieden sind die Befragten mit der Honorierung von Verbesserungsvorschlägen und der Transparenz von Aufstiegskriterien.

Abbildung 16: Zufriedenheit der Mitarbeiterlnnen in der aws

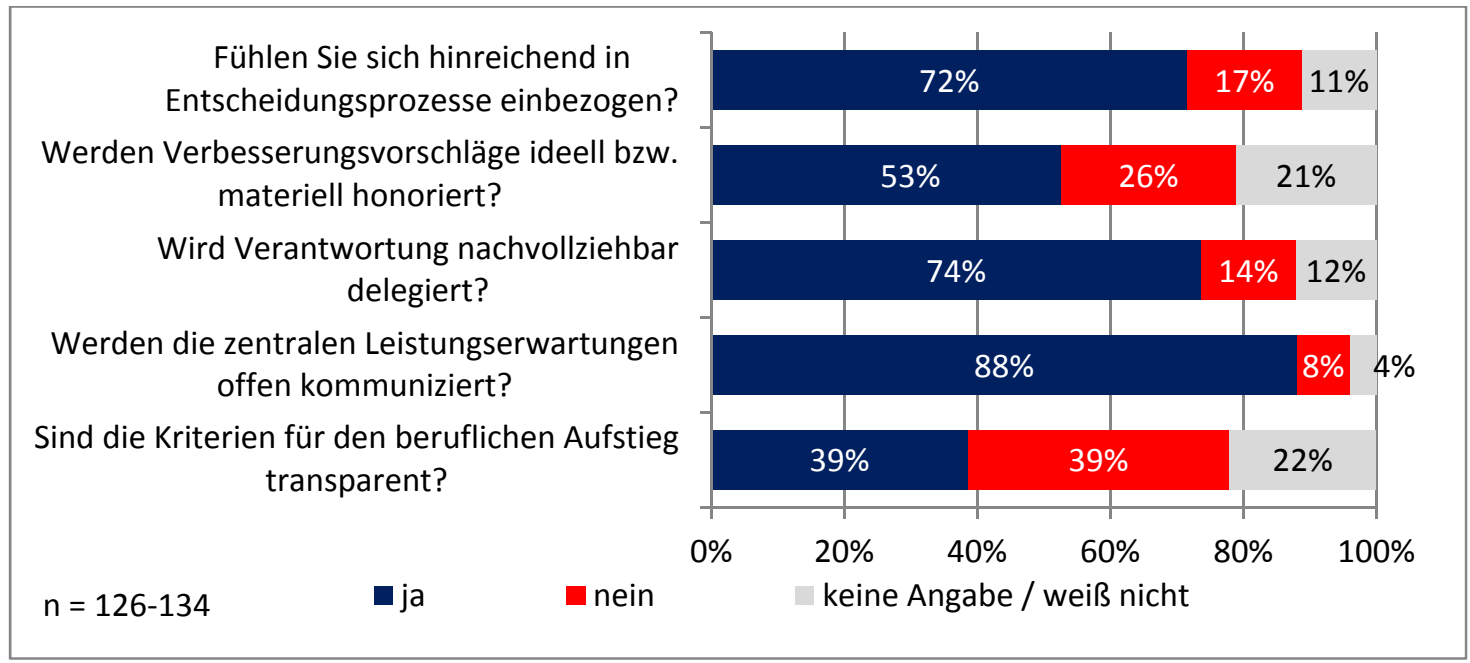


Die Agentur hat jedoch zahlreiche Personalentwicklungsmaßnahmen aufgesetzt, um die geringen Aufstiegsmöglichkeiten zu kompensieren. Diese resultieren in deutlich geringeren Fluktuationsraten im Vergleich zu der Zeit nach der Fusion. In der Organisationstheorie geht man davon aus, dass zwei bis drei Jahre nach einer Fusion die höchsten Abgänge zu verzeichnen sind, da dann die Organisation wieder neu ausgerichtet ist, alle Funktionen in der Regel besetzt und somit wichtige Weichen für die Zukunft gestellt sind. Dieser theoretische Ansatz bestätigt sich im Fall der aws. Die Fluktuationsrate betrug 2005 5,4 \%, schnellte 2006 auf $9 \%$ hoch und senkte sich dann sukzessive auf 3-4 \% ab. Diesen Wert hält die aws über die letzten 5 Jahre. 2013 verzeichnete sie davon abweichend mit 1,6\% den tiefsten Wert.

Bei der Frage, welche normativen Orientierungen die Tätigkeiten prägen, konstatieren die Befragten besonders ausgeprägt eine Orientierung an möglichst hoher Effizienz und guter Dokumentation; ein Ergebnis, das für eine Agentur, die für die öffentliche Hand tätig ist, jedoch naheliegend ist. Weiter kann festgehalten werden, dass die Ergebnisse der MitarbeiterInnen-Befragung zur Fehlerfreiheit Aufmerksamkeit in der Organisation verdienen, um konstruktiven Umgang mit Fehlern sicherzustellen. Im Rahmen des Validierungsworkshops wies die Leitungsebene der aws darauf hin, dass dies bereits geschehe.

Positiv hervorzuheben ist, dass die aws-MitarbeiterInnen mehrheitlich sehr zufrieden mit der Akzeptanz und Wertschätzung ihrer Arbeit sind. Hierbei spielen vermutlich verschiedene Möglichkeiten der Partizipation eine Rolle, z.B. im Rahmen der eigenen strategischen Arbeit (Mehrjahresprogramm, jährlich stattfindendes Zukunftsforum), die die MitarbeiterInnen mitnimmt und motiviert - hier werden auch Ideen für Programme geboren, z.B. aws First oder aws Integrate (im derzeitigen Plan), die dann in der Regel bei der Nationalstiftung eingereicht werden.

Das Zukunftsforum ist auch als Instrument zu sehen, mit dessen Hilfe Organisationsinnovation sichergestellt werden kann, indem dort in regelmäßigen Abständen geprüft wird, ob die aws zukunftsfähig aufgestellt ist. 
Abbildung 17: $\quad$ Normative Anforderungen und Wertschätzung in der aws

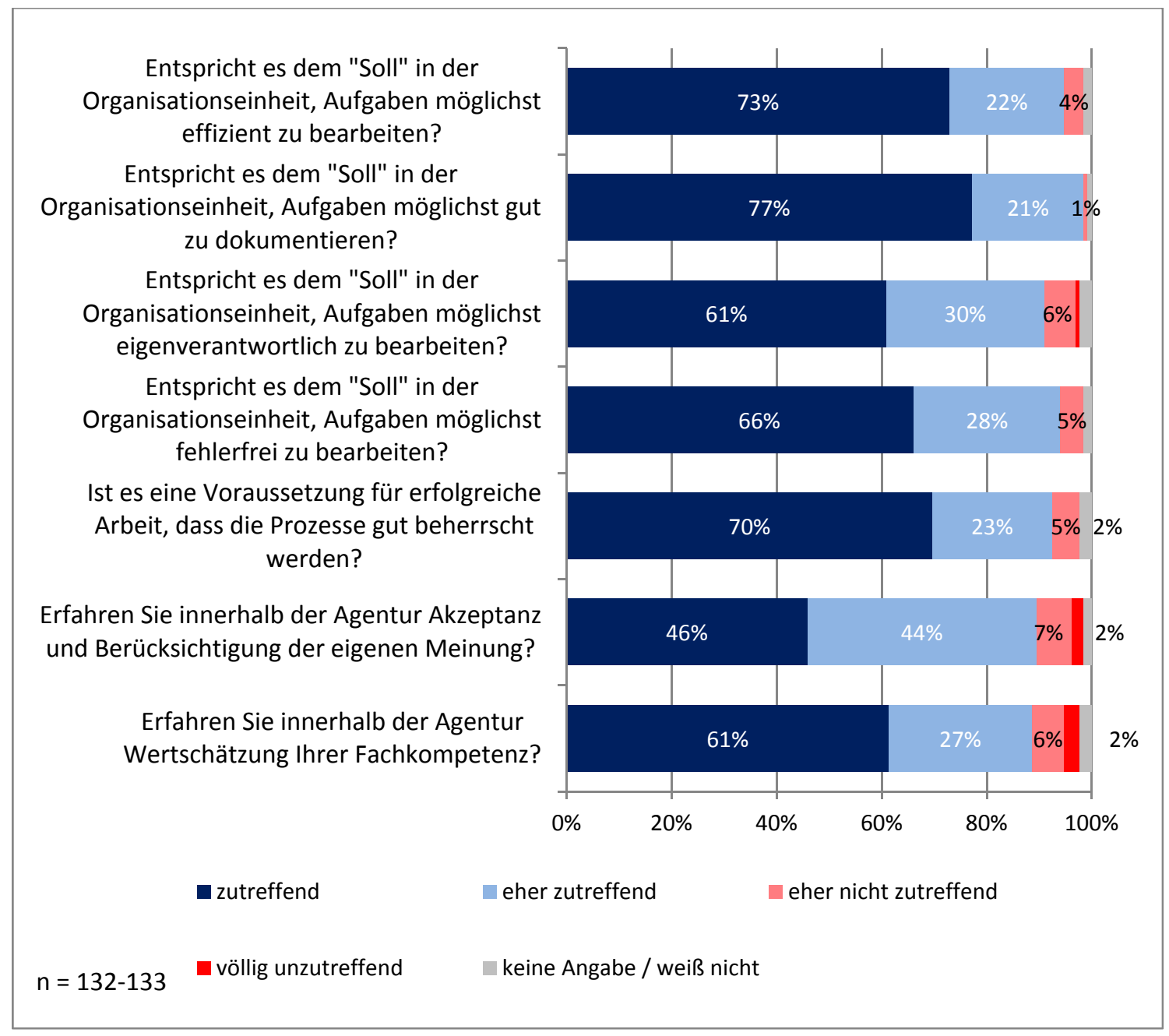

Es lassen sich kaum nennenswerte Unterschiede nach Bereichen, Vorgängerorganisation oder Aufgabenbereich feststellen.

\subsubsection{Management finanzieller Risiken}

Die aws hat ein internes Ratingsystem mit sieben Modulen zum Management des Kreditrisikos, um den unterschiedlichen Unternehmenstypen und Förderarten gerecht zu werden (beispielsweise KMU, Großunternehmen, GründerInnen, bilanzierende Kleinstunternehmen, innovations- und technologieorientierte Unternehmen, Projektfinanzierungen etc.). Dieses wurde von der Europäischen Kommission notifiziert. Laut Dokumentation der aws hat "die Qualitätssicherung des Ratingsystems durch die KPMG ergeben, dass alle untersuchten aws-Ratingmodule den Anforderungen des Leitfadens der OeNB und FMA sowie den branchenüblichen Standards entsprechen". 
Die aws finanziert in erster Linie Projekte, die das Unternehmen strukturell verändern sollen, daher berücksichtigt das Rating nicht nur Finanzkennzahlen (Hard Facts), sondern auch anderen Faktoren wie die vorhandene Ausbildung und Erfahrung im Unternehmen, das strategische Bewusstsein des Managements oder die Marktposition des Unternehmens (sog. Soft Facts). Zudem gibt es die Möglichkeit der Herabstufung des Ratings aufgrund von festgestellten Warnsignalen wie intransparente Strukturen oder Rechtsstreitigkeiten.

Laut Auskunft der aws wird "für alle Haftungen, Kredite und große Zuschüsse ein Erstrating vor Beschluss durch den Kundenbetreuer vorgenommen. Die Informationen (Bilanz und Soft Facts) werden von den Geschäftsbanken und bei größeren Projekten auch von den KundInnen direkt eingeholt." Es gibt monatliche sowie quartalsweise Monitoring-Berichte, die jeweils unter anderem die aktuelle bzw. erwartete Ausfallquote angeben. Eine wesentliche Herausforderung bestand für die aws bis vor kurzem darin, dass sie in den vergangenen Jahren zunehmend die geplanten Ausfallquoten (ca. 4-6\%) nicht ausschöpfen konnte.

Dies hatte einerseits damit zu tun, dass in Folge der Finanzkrise die Vorgaben für die Banken strenger und die Fälle immer risikoärmer wurden, weil bei Garantien die Banken auch einen Teil des Risikos nehmen müssen. Andererseits hatte dies aber auch damit zu tun, dass das BMF als Schadloshalter der aws-Garantien zunehmend risikoärmer agierte. Aufgrund eines Ministerratsbeschlusses vom Juli 2016 kündigt sich eine Veränderung dieser Situation an. Seit der Umsetzung des „aws Garantiepaketes“ kommt es zu einer Ausweitung der aws-Garantien (Neugarantievolumen) um 50\% jährlich. Damit wird erwartet, dass künftig wieder größere Risiken eingegangen werden.

\subsubsection{Projektauswahlkriterien}

Worauf legt die aws bei ihren Entscheidungen großen Wert? Vor allem auf eine hohe Chance zur Marktumsetzung, dicht gefolgt von der Standort-Stärkung. Dies ist das wesentliche Ergebnis aus der MitarbeiterInnenbefragung (siehe Abbildung 18). 
Abbildung 18: Förderkriterien aus Sicht der aws-Mitarbeiterlnnen

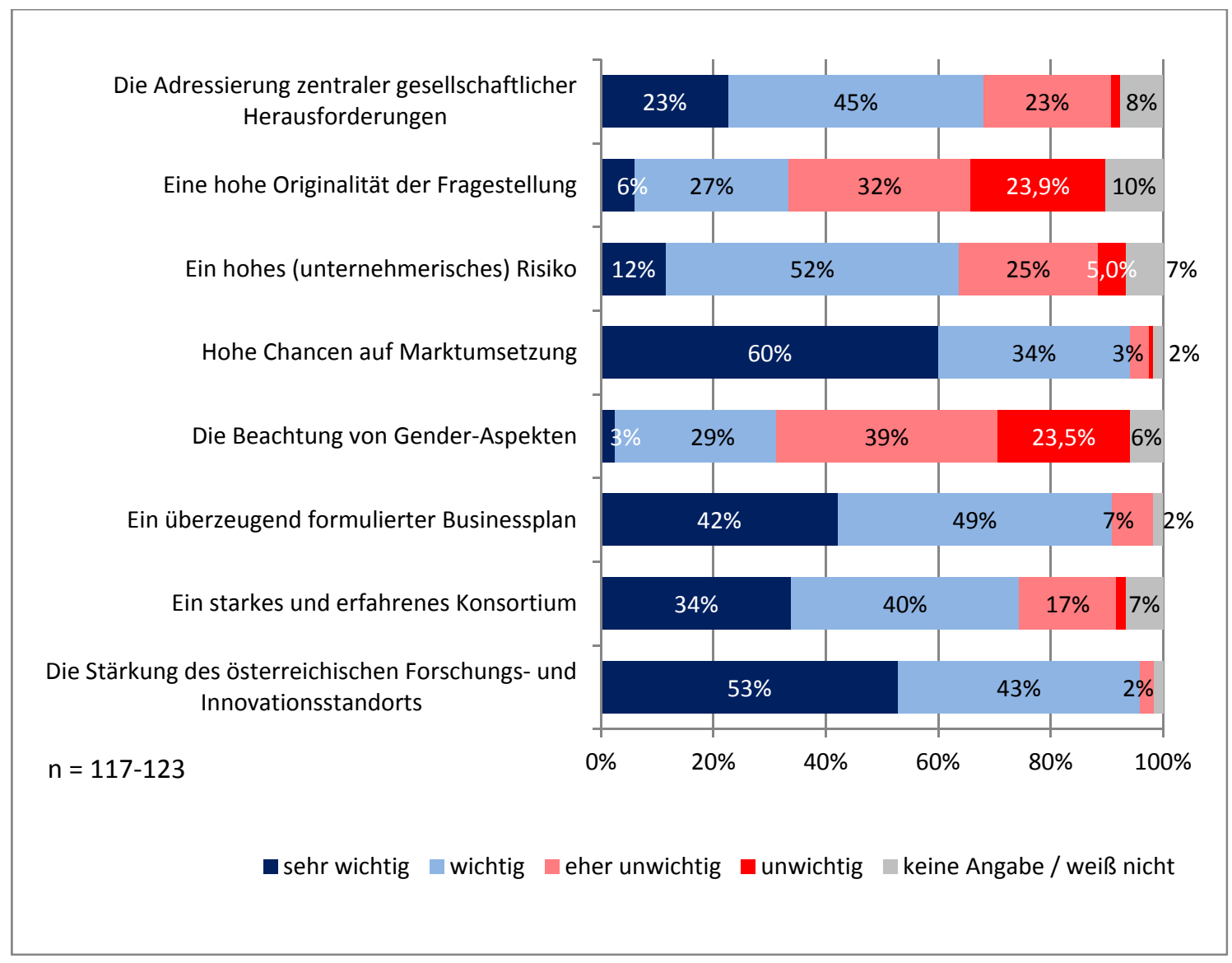

Die Antworten auf diese Fragen weisen eine Deckung mit dem aws-internen Instrument des "volkswirtschaftlichen Nutzens" auf. Dieses Instrument wird seit 2014 in der Agentur für die Antragsbeurteilung eingesetzt, sowie, um die Erträge der geförderten Projekte einheitlich messen und darstellen zu können. Kernelemente des Konzeptes sind Beiträge zum Wirtschaftswachstum (z.B. Beschäftigungseffekte, Investitionen) sowie die Innovationskraft des Unternehmens. Es gehören aber auch die Aspekte der Diversität (z.B. Gender) sowie der Umwelt hinzu, die die aws als „freiwillige Nebenziele“ deklariert. Wie die Zahlen der Befragung zeigen, werden diese beiden Kategorien bislang bei der Projektauswahl nicht als "sehr wichtig" eingestuft. Hierbei wird angenommen, dass Umweltschutz unter der Adressierung globaler Herausforderungen subsumiert wird, die von vielen aws-Beschäftigten als wichtiges Kriterium angesehen wird. Wenige kritische GesprächspartnerInnen haben angemerkt, dass ihrer Meinung nach das Konzept noch zu viele Spielräume lasse, so dass durchaus nicht nur die Spitzenprojekte zu sehr guten Bewertungen kommen könnten. Prinzipiell begrüßen sie das Konzept aber als wichtigen Schritt in die richtige Richtung. 


\subsection{Unternehmenskultur}

Auch für die aws sind wir zur Analyse der Unternehmenskultur der Frage nachgegangen, für was die Agentur aus Sicht ihrer MitarbeiterInnen steht und welche Adjektive sie mit der aws assoziieren.

Betrachtet man zunächst die Frage, für was die Agentur steht, so zeigt sich, dass am häufigsten (83 Nennungen) das Akronym herangezogen wird und ausgeführt wird, die aws stehe für "(Agentur/Partner für) Wirtschaftsförderung/Umsetzung der Wirtschaftsförderpolitik/Wirtschaftsförderung im Allgemeinen“. An zweithäufigster Stelle, jedoch mit 43 Nennungen nur etwa halb so häufig genannt, sind die Stichworte "Förderbank, Wirtschaftsförderung durch Kredite, Garantien, Beteiligungen u. Investitionen". An dritter Stelle schließlich folgt, in der Wahrnehmung der aws-MitarbeiterInnen, die Förderung und Unterstützung von Innovationen (28 Nennungen) sowie die Förderung und Unterstützung von Start-ups und Gründungen (19 Nennungen).

Neben den Aufgaben, die benannt wurden, erfolgten jedoch auch Qualitätszuschreibungen, beispielsweise indem die Kunden- und Serviceorientierung (25), die Effizienz, Effektivität und Wirksamkeit (15) sowie die Expertise, das Know-how und die Kompetenz ausgeflaggt wurden (12). Als Nennungen, die mehr als einmal vorkamen, sind noch Transparenz (7), Wertschätzung und Kollegialität (5), Engagement (3) und Nachhaltigkeit (2) anzuführen.

Die aws ist eine integrierte Organisation, hierzu haben der Leitbildprozess, TeambuildingMaßnahmen und nicht zuletzt der Umzug in die Walcherstraße beigetragen. Auf Führungsebene tragen die Management-Klausuren seit 2007 bzw. 2009 zur Integration bei. Und in der Tat wird bei der Analyse der Unternehmenskultur-Adjektive deutlich, dass diese einen sehr hohen Übereinstimmungsgrad mit dem Leitbild der aws aufweisen.

Abbildung 19: Unternehmenskultur aus Sicht der aws-MitarbeiterInnen

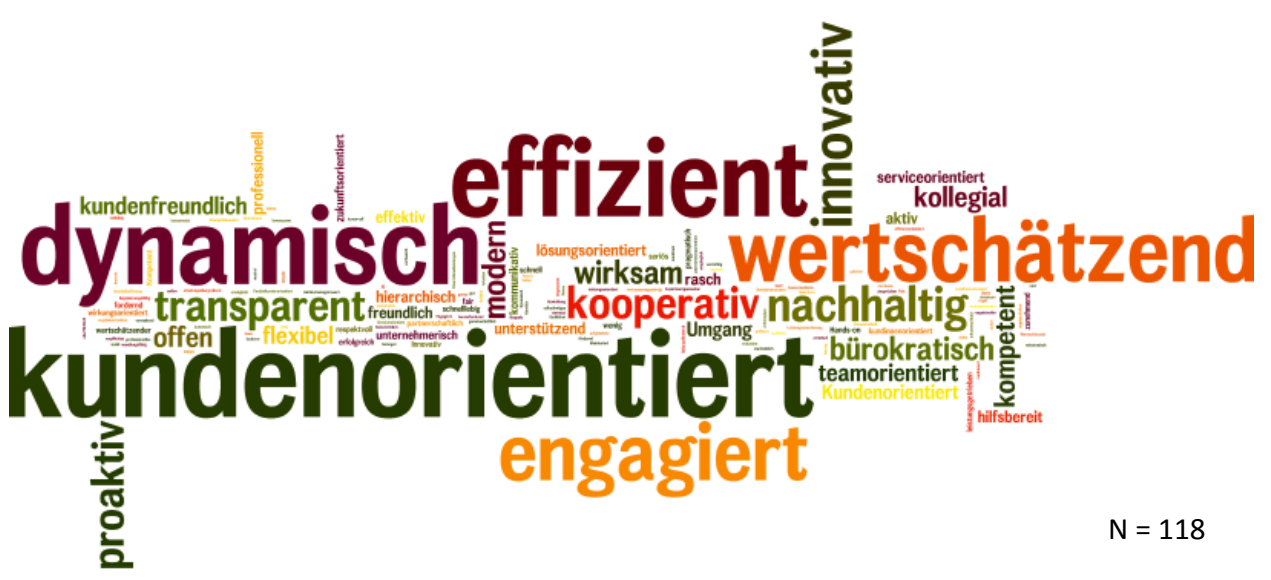


Anmerkung: N steht für die Zahl der Antwortenden, nicht für die Zahl der genannten Adjektive. Bei den word clouds werden auch Einzelnennungen gezählt. Je häufiger ein und derselbe Begriff genannt wird, desto größer erscheint er. Die Position der Adjektive wird per Algorithmus bestimmt und hat keine tiefer gehende Bedeutung.

In allen drei Förderbereichen steht die Kundenorientierung als Selbstzuschreibung im Vordergrund, die Bereiche Kredite und Garantien benennen darüber hinaus häufig die Effizienz als zentrales Merkmal Ihres Tuns, der Bereich Entrepreneurship das Wertschätzende.

Abbildung 20: $\quad$ Leitbild der aws

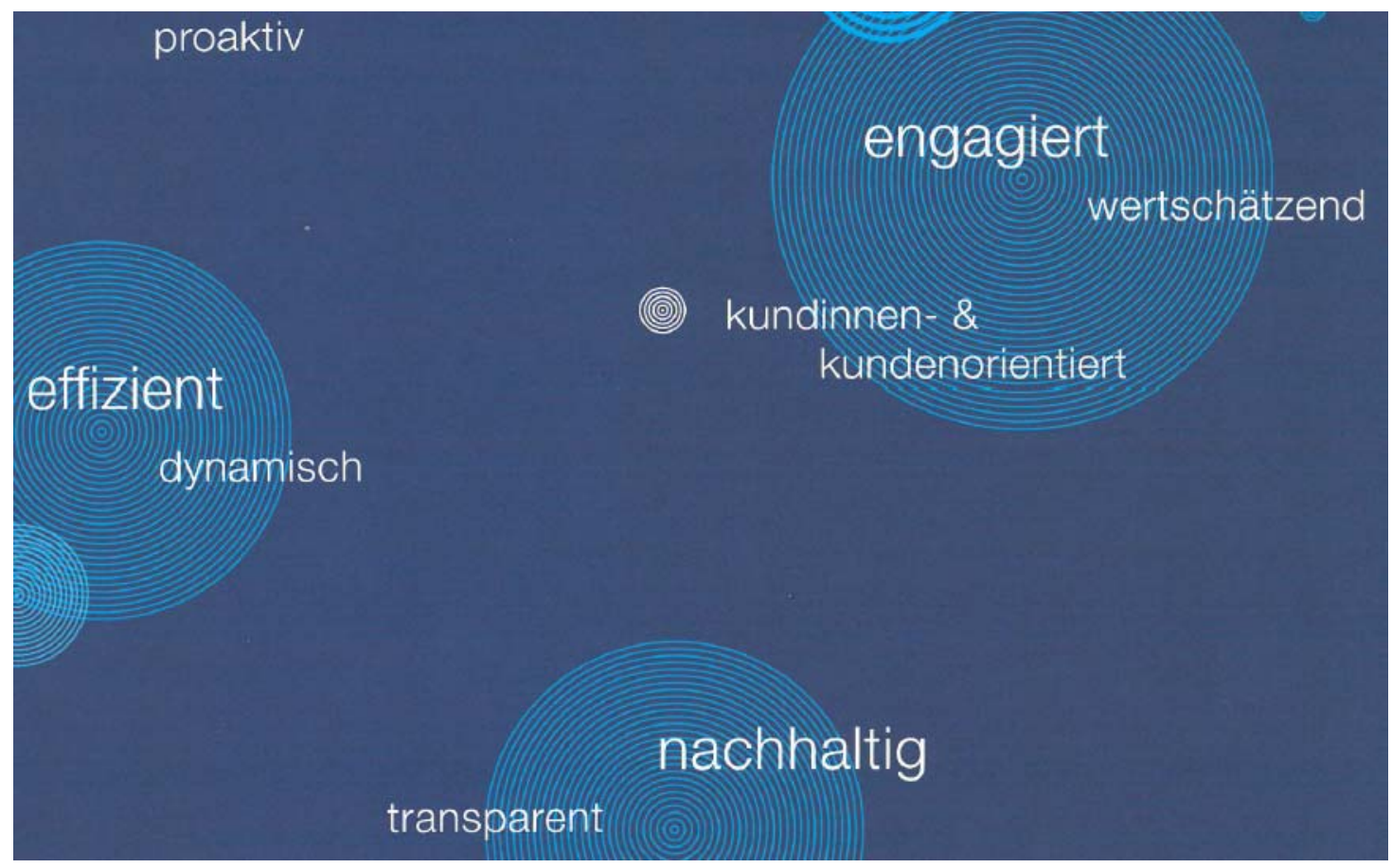

Nach Vorläuferorganisationen lässt sich feststellen, dass hier teilweise eigene Schwerpunksetzungen vorliegen: Vor allem MitarbeiterInnen aus der ehemaligen Innovationsagentur flaggen Begrifflichkeiten wie "modern", "kompetent" und "aktiv" aus, während für ehemalige BÜRGES-Beschäftige die Effizienz ein wichtiges Merkmal ihrer Unternehmenskultur darstellt. Gemeinsamer Kern ist aber für alle Vorgängerorganisationen die Kundenorientierung.

Festgestellte Unterschiede in der Unternehmenskultur nach Vorgängerorganisationen sollten nicht allzu kritisch gesehen werden. In den Gesprächen mit aws-Beschäftigten wurde festgehalten, dass man Angehörige von Vorgängerorganisationen durchaus auch heute noch am Sprachgebrauch erkennen könne. Tiefgreifende Kulturunterschiede, die zu Schwierigkeiten in der Zusammenarbeit führen könnten, liegen jedoch keinesfalls vor. 
Der hohe Stellenwert, der der Dynamik sowohl im Leitbild der aws als auch in der MitarbeiterInnenbefragung zukommt, wird auch durch die geführten Interviews in der aws bestätigt. Schnelligkeit in Bezug auf die Erarbeitung und Umsetzung von Kundenlösungen sowie in den internen Prozessen und im Austausch mit den Auftraggebern ist eine wichtige handlungsleitende Orientierung. Dies ist auch darauf zurückzuführen, dass die aws viele Personen aus der Privatwirtschaft einstellt. Die aws sieht sich heute auch oder vorwiegend als Dienstleister für Förderung, und nicht mehr als reine Expertenorganisation.

\subsection{Erreichung der Zielgruppen}

Die Befragten innerhalb der aws konstatieren mehrheitlich, dass sich ihr Verhältnis zu den Zielgruppen, die Erreichung potentieller Zielgruppen, aber auch die Erreichung programmspezifischer Förderziele deutlich bzw. eher vergrößert haben.

Abbildung 21: $\quad$ Veränderung der Zielgruppenerreichung seit der Fusion zur aws

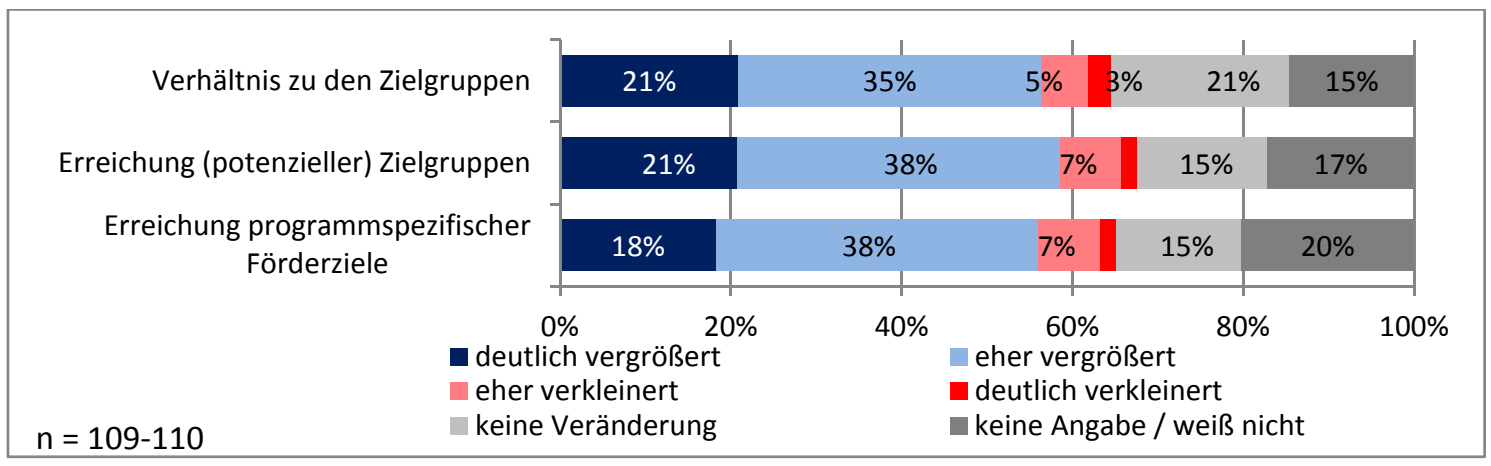

Was das Verhältnis zu den Zielgruppen angeht, so zeigt die Kundenbefragung der aws, dass die Fördernehmer die Professionalität, Kompetenz und Kundenorientierung als ausgezeichnet bewerten. Hierin spiegelt sich die hohe Kundenorientierung der aws, die zahlreiche Schritte unternommen hat (z.B. Kundencenter eingerichtet, Homepage auf die Bedürfnisse der Zielgruppen ausgerichtet), um die Zielgruppe gut zu adressieren, wieder. Positiv hervorzuheben ist, dass auch die Veränderung durch die Zusammenlegung der Organisationen hinsichtlich fünf von sechs Antwortkategorien überwiegend positiv eingeschätzt wird, bis auf den administrativen Aufwand, der aber generell gestiegen ist. 
Abbildung 22: Einschätzung der aws hinsichtlich ausgewählter Qualitätsmerkmale

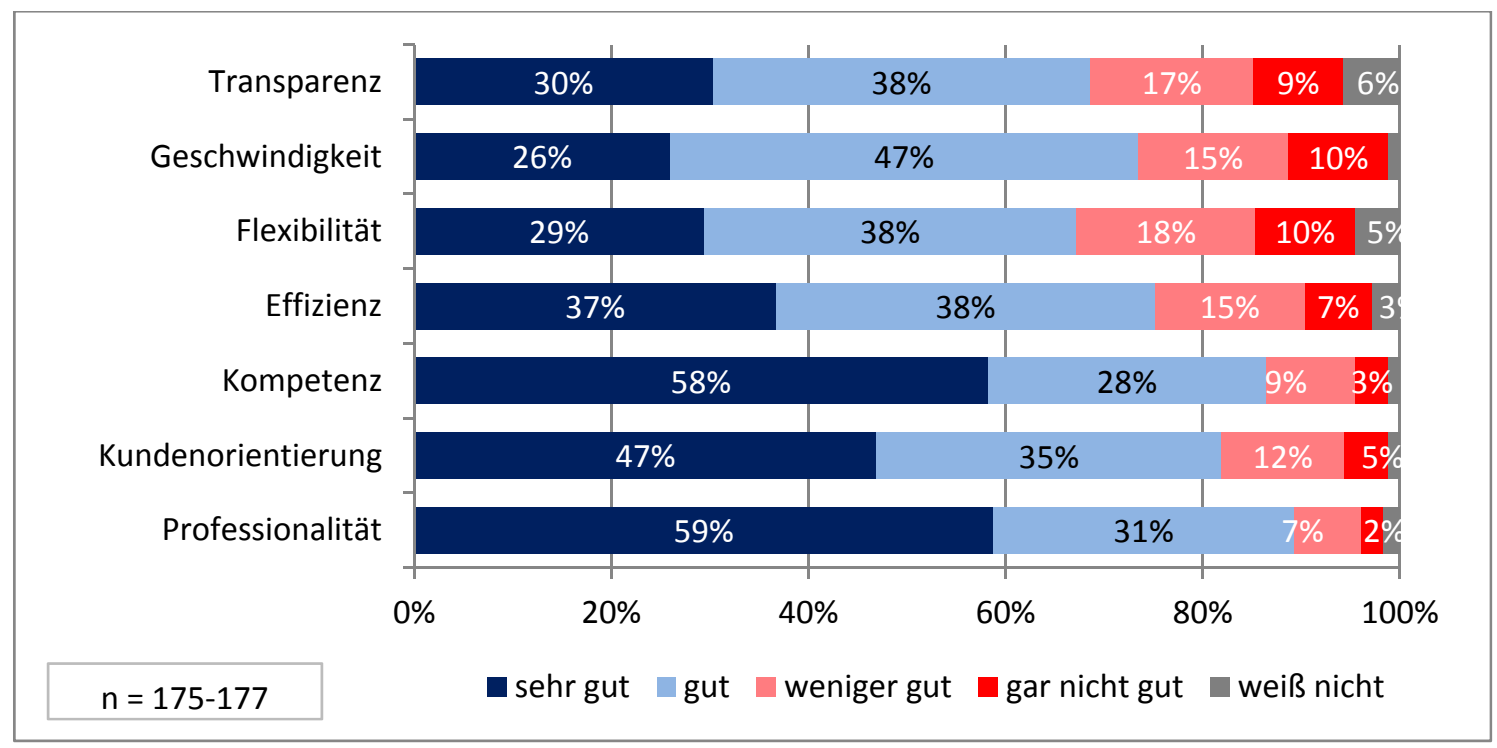

Als Herausforderungen zeigen sich, dass jeder vierte Befragte Transparenz, Flexibilität und/oder Geschwindigkeit der Entscheidungsprozesse kritisiert. Insbesondere an der Geschwindigkeit der Prozesse arbeitet die aws - diese ist einer der Key Performance Indicators der internen Steuerung. Allerdings sind bei einer großen Anzahl von Förderentscheidungen auch die Ministerien formal beteiligt (dies ist insbesondere im Fall der Garantien das BMF, bei Förderungen im Innovationsbereich das bmwfw), so dass die Dauer von Entscheidungsprozessen über Förderanträge nicht allein im Einflussbereich der aws liegt.

Abbildung 23: Beurteilung der Veränderung durch die aws-Fusion

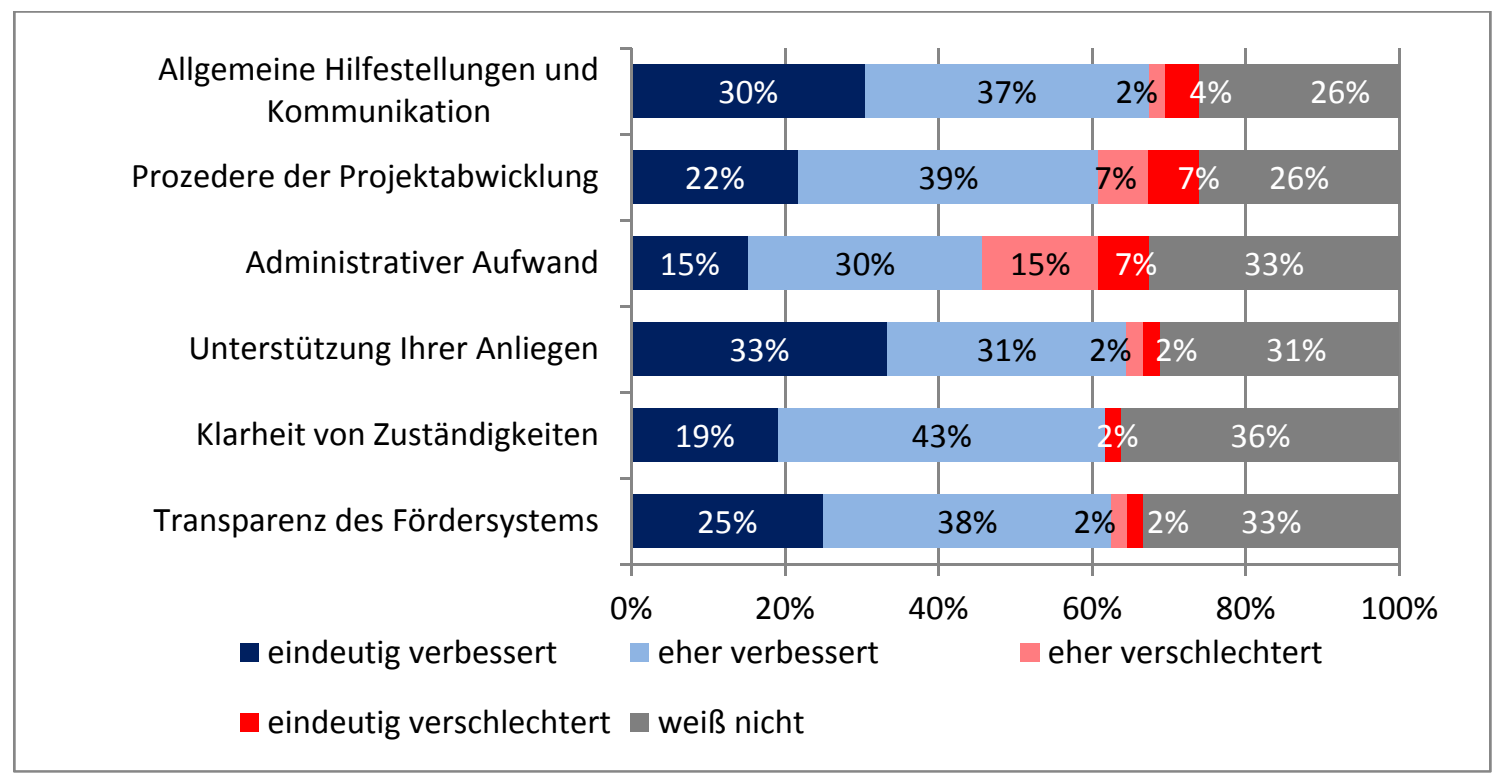


Einem Drittel der FörderkundInnen fällt es zudem schwer, die passende Unterstützungsmaßnahme zu identifizieren. In diesem Ergebnis schlagen sich die jüngsten Bemühungen zur verbesserten Beratung (s.o.) noch nicht nieder. Die aws hat im Rahmen des Validierungsworkshops klar zum Ausdruck gebracht, dass sie ihre bisherigen Bemühungen noch weiter intensivieren will.

Abbildung 24: Wie einfach oder kompliziert ist es aus Ihrer Sicht, die für Ihr Anliegen am besten geeignete Unterstützungsmaßnahme bei der aws zu finden?

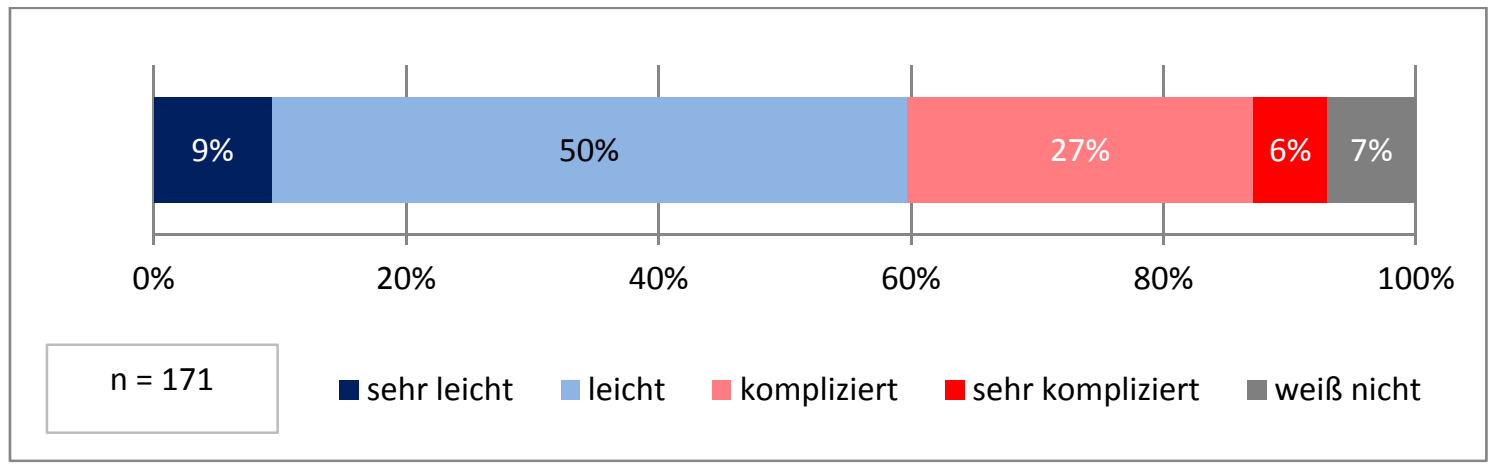

\subsection{Fazit}

Für die aws lässt sich zunächst festhalten, dass ihre Startbedingungen nicht optimal waren: die Fusion gilt als überstürzt durchgeführt und als mit unrealistischen Zielsetzungen überfrachtet. Auch die Heterogenität der Vorläuferorganisationen war hoch, es finden sich unter dem Dach der aws nicht nur eine Förderbank, sondern auch eine Förderagentur mit dem ehemaligen Portfolio der Innovationsagentur. Hinzu kamen in den Anfangsjahren mehrere Wechsel in der Geschäftsführung, die eine kontinuierliche Entwicklung der Agentur erschwert haben. Erst in den vergangenen ca. fünf bis acht Jahren sind zahlreiche und intensive Anstrengungen unternommen worden, ein gemeinsames Leitbild für die Organisation zu entwickeln und effiziente Strukturen und Prozesse zu implementieren. Mit Erfolg, wie sich zeigen lässt: Die aws erzielt nicht nur hohe Zufriedenheitswerte unter ihren MitarbeiterInnen, insbesondere ihre KundInnen äußern sich sehr positiv nicht nur gegenüber den Veränderungen im Vergleich zur Situation vor der Fusion, sondern auch hinsichtlich zentraler Qualitätsmerkmale wie Professionalität, Kompetenz und Kundenorientierung. Der aws kann insgesamt ein hohes Know-how bei der Fördermittelvergabe wie auch beim Bankgeschäft attestiert werden, sie verfügt über alle Werkzeuge, die man für diese Diversität an Tätigkeiten benötigt. Wenige kritische Stimmen inner- und außerhalb der aws bescheinigen ihr, dass sie bei der Fördermittelvergabe noch stringenter die in den letzten Jahren eigens entwickelten Tools zur An- 
wendung bringen sollte. Insbesondere das neue Konzept des "volkswirtschaftlichen Nutzens", das für die Antrags- und Erfolgsbewertung der Projekte entwickelt wurde, sollte künftig bei der Bewertung der Projektanträge strenger angewandt werden und weniger Spielräume lassen.

Trotz der skizzierten Diversität, neben dem Kerngeschäft Kredite, Garantien und Zuschüsse sowie Eigenkapital-Instrumente und Beratung kommt auch noch die Abwicklung von Wettbewerben, hat sich die aws klar in der Funktion Unternehmens- und Wirtschaftsförderung positioniert. Gleichwohl gilt die Bekanntheit der "Marke aws", vor allem im Vergleich zur früheren BÜRGES, als ausbaufähig. Dies hängt auch damit zusammen, dass Kredite und Garantien in der Regel über die Hausbanken abgewickelt werden, und hier oft kein direkter Kontakt zwischen KundInnen und aws stattfindet. Zur Steigerung der Bekanntheit und der corporate identity wurden jedoch bereits zahlreiche Schritte unternommen, wie oben ausgeführt wurde. Hier sollte in den kommenden Jahren nachverfolgt werden, ob diese Maßnahmen zu einer Verbesserung geführt haben.

Die aws agiert dank ihrer Effizienz- und Prozessorientierung hoch professionell. Sie hat in den vergangenen Jahren sehr viel Aufwand in die interne Organisationsentwicklung gesteckt und einen deutlichen Modernisierungsprozess befördert, inklusive der Implementierung einer Vielzahl moderner Management-Instrumente. Dies hat insgesamt zu optimierten und damit effizienteren Prozessen geführt.

Gleichwohl gibt es durchaus einzelne Stimmen unter den MitarbeiterInnen, die sich eine bessere ideelle bzw. materielle Honorierung von Verbesserungsvorschlägen wünschen und auf eine zu starke Zahlenorientierung bzw. ein Zuviel an Management-Tools hinweisen. Insgesamt besteht ein starker Fokus auf Effizienz. Weiter kann festgehalten werden, dass die aus einer Prozessorientierung resultierende Neigung zur Fehlervermeidung auch für die aws eine permanente Herausforderung darstellt, für die die aws-Verantwortlichen derzeit bereits eine gewisse Aufmerksamkeit haben. Die permanente Aufgabe dürfte weiterhin darin bestehen, eine Kultur des konstruktiven Umgangs mit Fehlern weiter zu pflegen.

Die aws kann zweifellos als integrierte Organisation bezeichnet werden, hierzu haben insbesondere der Leitbildprozess, Teambuilding-Maßnahmen, der Umzug in die Walcherstraße, aber auch die Ende der Nuller-Jahre eingeführten Management-Klausuren beigetragen. Die Analyse der Unternehmenskultur-Adjektive macht denn auch deutlich, dass diese einen sehr hohen Übereinstimmungsgrad mit dem Leitbild der aws aufweisen. Der hohe Stellenwert, der der Kunden- und Serviceorientierung, und hier insbesondere der Dynamik, sowohl im Leitbild der aws als auch in der Mitarbeiterlnnenbefragung zukommt, wird auch durch die geführten Interviews in der aws bestätigt. Diese zeigen, 
dass Schnelligkeit in Bezug auf die Erarbeitung und Umsetzung von Kundenlösungen sowie in den internen Prozessen und im Austausch mit den Auftraggebern eine wichtige handlungsleitende Orientierung ist. Dies begründet sich auch darin, dass die aws viele ihrer MitarbeiterInnen aus der Wirtschaft rekrutiert, die diese Kultur bereits mitbringen.

Mit Blick auf die Erreichung der Ziele der Strukturreformen lassen sich zusammenfassend folgende Aussagen treffen:

- Reduzierung der organisatorischen und inhaltlichen Komplexität: Hier verhindern stark unterschiedliche Finanzierungssettings die Vereinheitlichung von Steuerung, z.B. die Komplexität der aws Garantieprogramme mit ihrer Verflechtung von Auftraggebern, Eigentümern, Budgetkontrolle durch das BMF und dem Verbleib des Ausfallsrisikos ebenfalls im Finanzministerium.

- Lösung von Koordinationsproblemen: Dieses Ziel wurde kaum erreicht, für die aws besteht vielmehr weiterhin die komplexe Situation, insbesondere im Hinblick auf die Abwicklung der Garantien, da hier auch das Finanzministerium eine wichtige Rolle spielt.

- Erhöhung der politischen Steuerungsfähigkeit: Abgeleitet aus Herausforderungen der Koordination gilt auch für die Steuerungsfähigkeit, dass diese insgesamt ausbaufähig erscheint.

- Umsetzungschancen des Regierungshandelns: Dieses Ziel kann als weitgehend erreicht angesehen werden: Die Angebote der aws - neben den Zuschüssen vor allem die Kredite und Garantien, deren Konditionen, die tilgungsfreien Jahre, das Ausmaß der Risikoteilung - sind von hoher Relevanz für die Industrie und stoßen grosso modo auf eine gute Nachfrage. Außerdem wird die Agentur auch von ihren Zielgruppen sehr positiv bewertet, nicht nur im Rahmen der Online-Erhebung, sondern auch in den durchgeführten Unternehmensinterviews.

Als Herausforderung für die nähere Zukunft bleibt aber sicherlich die seit längerem anhaltende Niedrigzinsphase bestehen, die einige der aws-Angebote weniger attraktiv gemacht haben, da deren Geschäftsmodell durchaus auch von der Zinshöhe abhängt. 


\section{$6 \quad$ Positionierung und Koordination der Agenturen}

Dieses Kapitel fokussiert auf die Positionierung der beiden Agenturen FFG und aws im Kontext der Strukturreform sowie ihre Koordination untereinander und mit anderen Förderorganisationen. In diesem Zusammenhang waren die folgenden Evaluierungsfragen für die empirische Arbeit handlungsleitend:

\section{Positionierung}

- Wie hat sich die Positionierung der FFG und der aws im Vergleich zur Situation vor der Strukturreform sowie nachher im Kontext zu anderen nationalen Institutionen sowie Institutionen auf Bundesländerebene entwickelt (qua Funktion, qua Größe)?

- Was bedeuten die Erweiterungen der Tätigkeit für Dritte (wie Bundesländer) sowie die Ausweitung des Handelns (u.a. EU-Monitoring) neben den ursprünglichen Kernaufgaben für die Positionierung von FFG und aws?

- Welche institutionellen Verknüpfungen der beiden Agenturen existieren über deren ursprüngliche Tätigkeit sowie über deren Organe? Welche Auswirkungen haben diese auf die Steuerbarkeit der Agenturen und auf die Strategiefähigkeit?

\section{Koordination}

- Wurde eine stärkere (horizontale) Koordination zwischen den Agenturen im österreichischen Förderungssystem durch die Reduktion von deren Anzahl bei gleichzeitiger gestiegener Größe/Komplexität der Agenturen erreicht?

- Welche Schnittstellen bzw. Abgrenzungen der Förderungsgesellschaften FFG und aws zueinander können identifiziert werden? Wie werden sie gemanagt?

- Wie gehen FFG und aws vor, wenn FörderungswerberInnen bei beiden Agenturen Projekte gleichzeitig oder hintereinander beantragen? Wurden Synergie- und Vereinfachungspotentiale umgesetzt?

- Erfolgt ein organisationales Lernen (bezüglich Prozesse und Strukturen) voneinander?

\section{Lessons learned}

- Welche Lehren lässt sich hinsichtlich der Steuerfähigkeit sowie der Steuerbarkeit der Agenturen ziehen?

- Welche Verbesserungspotenziale lassen sich mit Blick auf die Ziele der Strukturreform im Bereich "Lösung immanenter Koordinationsprobleme durch die Schaffung von ressortübergreifenden Förderungsgesellschaften" identifizieren?

- Auf welchen Ebenen und mit welchen zeitlichen und personellen Eckpunkten ist eine horizontale Koordination zwischen FFG und aws anzustreben? 


\subsection{Ausgangssituation}

Eines der wesentlichen Ziele, die zur Strukturreform im Förderungsbereich führten, war die "Lösung immanenter Koordinationsprobleme durch die Schaffung von ressortübergreifenden Förderungsgesellschaften." Eine Annahme bei Gründung der FFG und der aws war die wahrgenommene Inkompatibilität der insgesamt acht Finanzierungs-, Förderungs- und Beratungseinrichtungen, welche die Koordination der Organisationen untereinander erschwerte. Dies stellte eine bedeutende Herausforderung hinsichtlich der Zielgruppenansprache sowie der Standardisierung der Servicequalität dar.

Durch die Zusammenführung und Bündelung der unterschiedlichen Institutionen in die FFG und die aws sollte eine verbesserte Koordination erreicht werden. Ein derartiger Prozess wird sowohl von der formellen Organisationsreform als auch von vielschichtigen informellen Prozessen innerhalb der Agenturen wie auch seitens der Eigentümer und Auftraggeber beeinflusst, und zieht aufgrund dessen unterschiedliche interne Maßnahmen und kulturelle Entwicklungen nach sich (siehe Kapitel 4 und 5 zu den beiden Agenturen).

Die unterschiedliche Positionierung der Agenturen im österreichischen Fördersystem ist genauso aus der historischen Perspektive ihrer Vorläuferorganisationen zu betrachten, wie auch im Kontext der Entwicklung ihrer Beziehung zu regionalen und anderen nationalen Institutionen, aber auch im internationalen Kontext. Hier kam es seit der Gründung zu zusätzlichen Beauftragungen und Aktivitäten (FFG: z.B. EU-Performance Monitoring, Begutachtung der Forschungsprämie, internationale Programme, EFRE, Vertretung der Interessen der Ministerien in internationalen Initiativen, Präsenz im TAFTIE Netzwerk; aws: z.B. EFRE, Präsenz in der European Association of Guarantee Institutions).

\subsection{Positionierung der Agenturen}

Die aws konzentriert sich auf die zwei Schwerpunkte "Gründung" sowie "Wachstum und Industrie". Innerhalb dieser zwei Schwerpunkte liegt der Fokus auf der Unterstützung von Investitionen entlang der fünf Themen (1) Entrepreneurial Spirit (Vor-Gründung), (2) Technologieverwertung, (3) Gründung, (4) Einführung neuer Produkte und Dienstleistungen sowie (5) Wachstumssprünge. Damit wird versucht, wichtige Phasen, von der Vor-Gründung über die Gründung bis hin zur Internationalisierung von Unternehmen anzusprechen. 
Abbildung 25: Verbindung der aws-Schwerpunkte mit den Wirkungszielen

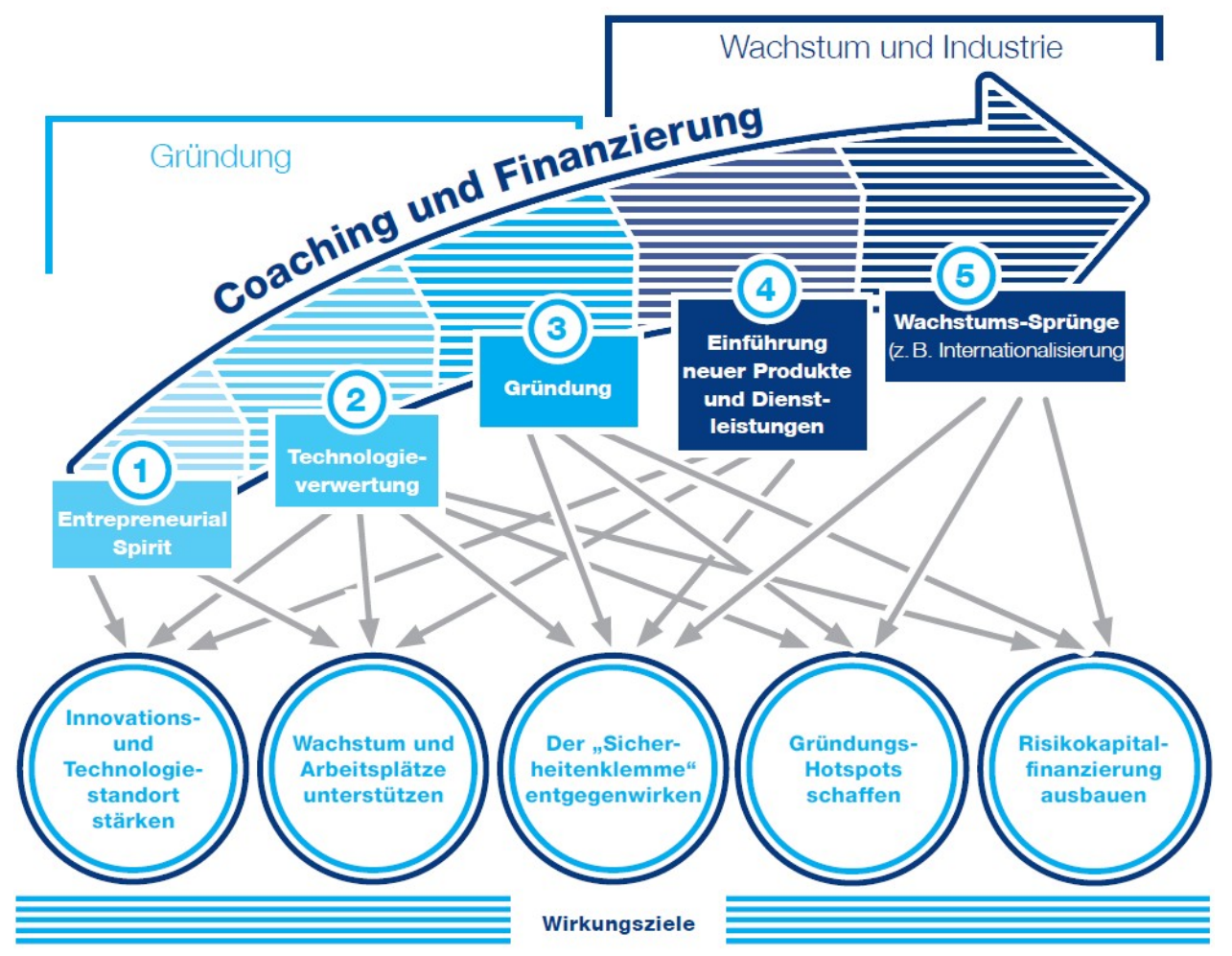

Quelle: aws Mehrjahresprogramm 2017-19, S. 28

Der aws steht hierbei eine breite Palette an Instrumenten zur Verfügung: Kredite, Zuschüsse, Garantien, Beteiligungen sowie Service und Beratung. Auch wenn die aws schlussendlich, vergleichbar mit der FFG, Projekte finanziert, steht bei der aws vermehrt das Unternehmen als Ganzes im Vordergrund. Dies trifft nicht nur bei Gründungsunternehmen zu, sondern auch bei der Unterstützung von Internationalisierungsschritten von etablierten Unternehmen aufgrund der Vergabe von Krediten und Garantien.

Die FFG fokussiert hingegen primär auf angewandte Forschungs- und Entwicklungsprojekte sowie auf die Forschungskooperationen zwischen Wissenschaft und Wirtschaft. Hierzu hat sich die FFG eine instrumentenspezifische Struktur erarbeitet, in der ein breitgefächertes Programmportfolio von FuE-Projekten in den Instrumentengruppen (1) Einstieg, (2), FEI-(Einzel- und Kooperations)-Projekte, (3) Strukturen und Netzwerke, (4) Personenförderung, (5) FEl-Aufwendungen und (6) Markteinführung zusammengefasst ist. Dabei werden die folgenden Wirkungsfelder adressiert: 
Abbildung 26: Die Wirkungsfelder der FFG gemäß FTI und FFG Richtlinien

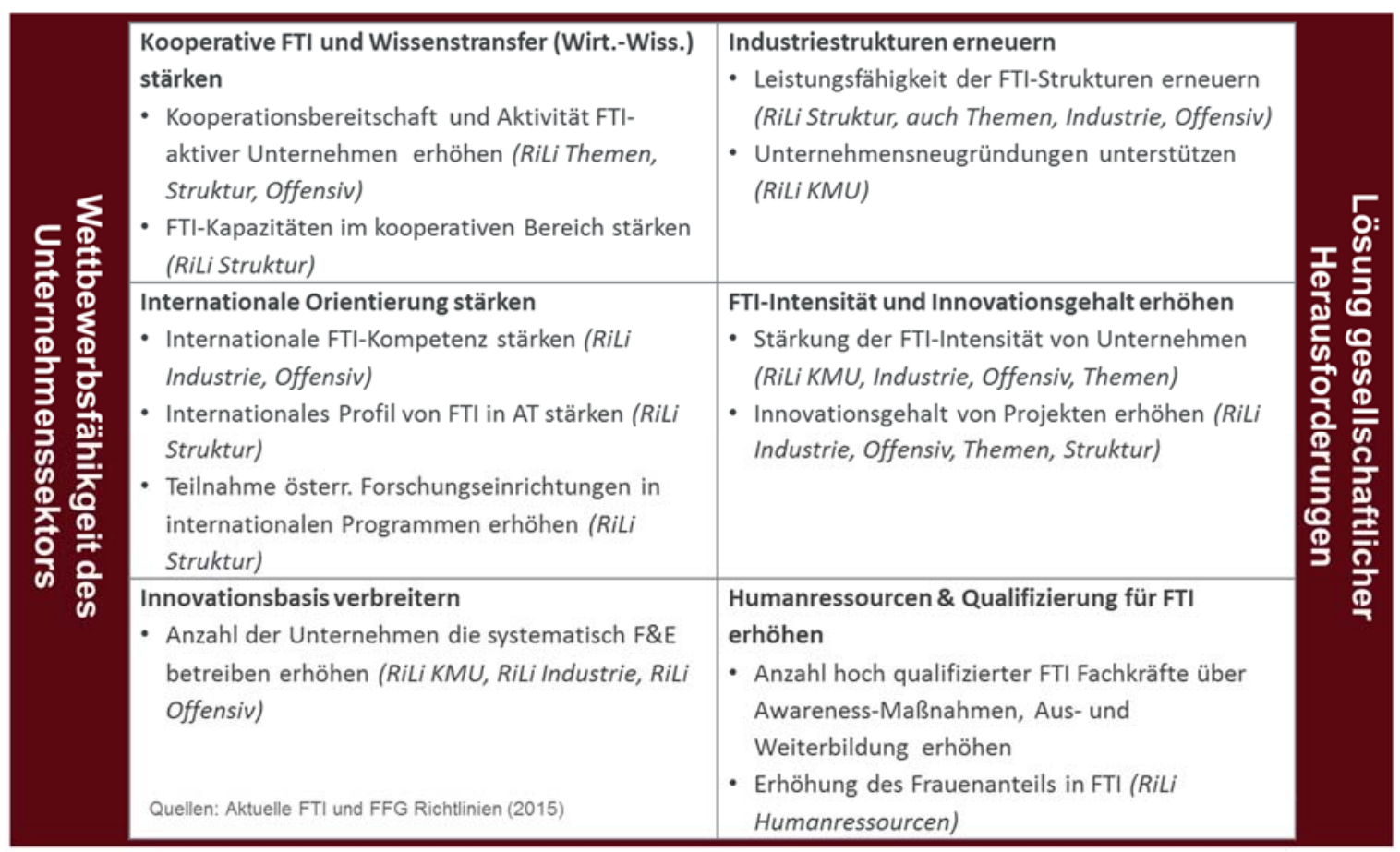

Quelle: Dinges et al. (2016). Indikatoren zur Erfassung der Wirkungen von Förderungen der FFG. Im Auftrag der Forschungsförderungsgesellschaft, Wien

Auch der FFG steht hierzu eine große Bandbreite an Unterstützungsmöglichkeiten zur Verfügung (§2 FFG-G), die ein flexibles Design von Interventionen ermöglicht. Eine deutliche Abgrenzung zur aws besteht dabei im Fokus auf anwendungsorientierte FuE-Projekte (industrielle Forschung und experimentelle Entwicklung), wobei die Bonität der Unternehmen bei der FFG zwar in Bezug auf die Beurteilung von Projektanträgen berücksichtigt wird, jedoch im Unterschied zur aws im Hintergrund bleibt. 5 In den letzten Jahren hat sich der Fokus der unmittelbaren Unterstützung der FFG über Unternehmen hinaus verstärkt auf das wissenschaftliche System erweitert. Dies bedeutet, dass der FuE-Charakter der Unterstützung durch die FFG überwiegend beibehalten wird. Es existieren jedoch auch Überlappungsbereiche in Bezug auf die Aktivtäten der beiden Agenturen, worauf weiter unten noch näher eingegangen wird.

Laut Einschätzung der interviewten ExpertInnen sind die Profile von FFG und aws, von wenigen Beispielen abgesehen, hinreichend unterschiedlich: beide Agenturen haben ei-

5 Die Ausnahme stellt das Basisprogramm mit der Vergabe von Zuschüssen, Krediten und manchmal auch Garantien dar. 
nen gut definierten USP (FuE Projekte liegen bei der FFG, investive Unternehmensförderung bei der aws). Aus diesen unterschiedlichen Blickwinkeln unterstützen beide Agenturen innovative Projekte.

Darüber hinaus sind die beiden Agenturen auch in vielfältige nationale und internationale Abstimmungsprozesse eingebunden. Einerseits erfolgte über die Jahre eine langsam wachende Zusammenarbeit mit den Bundesländern, indem - im Sinne eines top-up Zusatzfinanzierungen von einigen Bundesländern treuhänderisch übernommen, bzw. bei Garantien zusammen mit einer Landesorganisation im Paket unterstützt, oder Ausschreibungen zusammen mit vereinzelten Bundesländern durchgeführt wurden. Diese Art der Abstimmung, auch wenn sie im Konkreten doch relativ komplex ist, erhöht sicherlich die Effizienz der Abwicklung von Förderungen in Österreich und reduziert die Möglichkein von Doppelförderungen ein wenig, da Top-ups von nationalen Förderungen oder gemeinsame Ausschreibungen dies unterbinden. Hier gibt es jedoch noch ein großes Potenzial zur Erhöhung von Effektivität und Effizienz von Förderungen in Österreich, indem Bundesländer ihre Portfolios auf die nationalen Agenturen abstimmen bzw. von ihnen abwickeln lassen. Dies liegt aber nicht im Entscheidungsbereich der Agenturen oder des Bundes, sondern in jenem der Bundesländer.

Es gibt auch auf nationaler Ebene noch Organisationen, die komplementäre Aufgaben zu den beiden Agenturen durchführen. Bei der FFG ist vor allem der FWF zu nennen, der mit seinem Fokus auf Grundlagenforschung ein natürliches Naheverhältnis zur FFG aufweist, oder der Klima- und Energiefonds (KLIEN)6, der als Koordinationsstelle des Bundes die Themen Klimawandel und Energie adressiert, und sich unter anderem der beiden Agenturen zur Umsetzung von Programmen bedient. Hinsichtlich der aws stehen auf nationaler Ebene insbesondere Institute wie die Österreichische Hotel- und Tourismusbank (ÖHT) oder die Österreichische Kontrollbank (ÖKB, und die Österreichische Entwicklungsbank ÖEB) im Naheverhältnis, da diese ein ähnliches Portfolio bedienen, und mit den Geschäftsbanken auch denselben Vermarktungsweg nutzen.

Spezialfall missionsorientierte Programme: Einige der an den Grand Challenges ausgerichteten Förderprogramme weisen einen spezifischen Bedarf dahingehend auf, als Themen, und damit Forschungs-Communities im öffentlichen und privaten Bereich, und damit capabilities/Kompetenzen erst entwickelt werden müssen. Die Vernetzung sowie der

6 Der KLIEN vergibt Programme an FFG (Forschung), aws (Marktüberführung), Schienen- und Infrastrukturgesellschaft (SCHIG) und Kommunalkredit Public Consulting (KPC). Die Förderabwicklung wird jedoch aufgrund von nicht harmonisierten Prozessen verkompliziert. Hier liegt insofern ein Effizienzpotenzial, als Datenbanken und Berichtsdarstellungen für den KLIEN noch doppelt geführt werden. 
Wissenstransfer nehmen bei einigen missionsorientierten Programmen eine noch bedeutendere Rolle ein und sollten damit noch aktiver von den Agenturen unterstützt werden. Hier übernimmt z.B. die Österreichische Gesellschaft für Umwelt und Technik (ÖGUT) zum Thema Energie oder die AustriaTech zum Thema Mobilität teilweise die Mobilisierung der Community, inkl. des Veranstaltungsmanagements bzw. die Aufbereitung und Verbreitung von Forschungsergebnissen im Auftrag des bmvit. Darüber hinaus wird auch die Beratung für Förderungseinreichungen, ergänzend zur FFG, angeboten.

Im Rahmen der Erweiterungen der Tätigkeit für Dritte ist bei der FFG auch die Übernahme der Begutachtung der Forschungsprämienansuchen im Auftrag des BMF zu erwähnen. Für diese Aufgabe wurde ein umfangreicher Qualitätssicherungsprozess über weite Teile der FFG installiert, in den alle FFG ExpertInnen involviert sind, und geschätzte 40 - 80 Stunden pro ExpertIn und Jahr dafür aufwenden. Diesem doch bedeutenden Zusatzaufwand steht nach FFG-interner Sicht der Vorteil gegenüber, dass diese Zusammenarbeit über Bereiche hinweg einen weiteren Schritt in Richtung Standardisierung bzw. Qualitätssicherung für die FFG insgesamt mit sich brachte.

Die Übernahme des EU-Performance Monitorings durch die FFG (im Auftrag der beiden Eigentümerministerien sowie dem Lebensministerium) brachte es mit sich, dass nun über das Themenlabelling ein guter Überblick zu den FFG-internen und EU Aktivtäten gegeben ist. Durch die Verknüpfung von nationalen und EU-Daten wird in Zukunft eine noch tiefergehende Analyse von Förderdaten möglich sein.

Die beiden Agenturen sind auch international stark engagiert. Sei es über ihre internationalen Netzwerke von europäischen Schwesterorganisationen, um ein Lernen voneinander quer über Europa sicherzustellen, über die Unterstützung ihrer Eigentümer und Auftraggeber hinsichtlich der EU Agenden, oder, weil supranationale Inhalte das konkrete Arbeitsgebiet darstellen (H2020, ERA, EFRE, GIN, de-minimis-Prüfungen, JTIs, ESA Kooperationen, OSTA, europäischen NCP Projekte, Bilat Projekte Servicierung von bilateralen Abkommen, etc.). Aufgrund des im letzten Jahrzehnt stark gewachsenen Portfolios an internationalen Initiativen ist es als besonderer Vorteil zu werten, dass sich diese Aufgaben nun auf zwei Agenturen konzentrieren, und sich damit die organisatorische Komplexität in Grenzen hält.

Eine institutionelle Verknüpfung der beiden Agenturen FFG und aws kann einerseits über ihre Tätigkeit und andererseits über ihre Organe erfolgen. Hinsichtlich ihrer Tätigkeit sind diese Verknüpfungen vordringlich über gemeinsam administrierte Programme definiert. Hier sind die gemeinsame Plattform Global Incubator Network (GIN) sowie das Förderprogramm Frontrunner zu nennen. Die operative Abstimmung funktioniert hier 
entsprechend Aussagen der beiden Agenturen klaglos. Darüber hinaus erfolgt eine fallweise Abstimmung auch in anderen Bereichen (z.B. EFRE, Life Sciences), und es wurden im Jahr 2016 das Quick-Check-Verfahren und der Förderpilot eingeführt, damit sich KundInnen schneller und besser orientieren können und die Gefahr von Doppelförderungen zwischen den beiden Agenturen noch besser ausgeschlossen werden kann. Bis dato wurde eine Abstimmung auf bilateralem Wege per Telefon vorgenommen, oder über quartalsweise Jour-Fixes wie etwa zum Thema Life-Sciences. Antragssteller müssen im Antragformular angeben, ob bzw. wo weitere Projekte öffentlich unterstützt werden. Die Abstimmung ist hauptsächlich hinsichtlich der Einhaltung der de-minimis-Grenzen relevant. $^{7}$

In diesem Zusammenhang ist die österreichische Interpretation des Datenschutzes wenig hilfreich, da auch die Förderdaten der beiden Agenturen nicht zusammengeführt werden dürfen (wie auch die Förderdaten innerhalb der FFG bei direkter und indirekter Forschungsförderung nicht zusammengeführt werden dürfen). Hier ist eine Reform der Datenschutzgestion hinsichtlich der Ermöglichung einer Zusammenführung von Datenbeständen dringend anzuraten, um im Sinne der österreichischen Steuerzahlerlnnen eine effektive Kontrolle anhand einer besseren Datengrundlage umsetzen zu können (siehe auch Kapitel 10.2 Handlungsempfehlungen).

Eine institutionelle Verknüpfung der beiden Agenturen über ihre Organe ist nicht gegeben. Aus der Geschäftsführung der FFG war eine Person bis 2006 im Aufsichtsrat der aws aufgrund der früheren Verbindungen mit dem Eigentümerressort vertreten. Während die Aufsichtsratsvorsitzende der FFG beim FWF Aufsichtsrat als ,non-voting' Mitglied vertreten ist (und vice versa), wurde dies bislang hinsichtlich FFG und aws als nicht nötig erachtet. Innerhalb der FFG ist der stellvertretende Vorsitzende des Aufsichtsrats auch im Beirat des Basisprogramms vertreten. Zwischen der aws und dem ERP-Fonds gibt es insofern eine personelle Verflechtung, als die Geschäftsführer der aws mit jenen des ERP-Fonds identisch sind (Personalunion) und ein Mitglied des aws-Aufsichtsrats gleichzeitig Mitglied in der ERP-Kreditkommission ist.

In Bezug auf die Positionierung von Themen gegenüber den Kundlnnen zeigen die durchgeführten Befragungen, dass sich die FFG mit ihrem Kerngeschäft, der Unterstützung von anwendungsorientierten FuE-Projekten bei ihrem Kundenkreis gut positionieren konnte. Bei der aws ist der Befund etwas komplexer. Zwar sind die Wiedererkennungswerte bei Themen wie Investitionsförderung, Garantien und Start-up gegeben, jedoch auf einem im Vergleich niedrigeren Niveau. Dies dürfte zumindest drei Ursachen

7 Eine österreichweite Prüfung der de-minimis-Fördergrenzen würde jedoch eine Komplettierung der Transparenzdatenbank um die vollständigen Einträge der Bundesländer benötigen. 
haben. Erstens sind Produkte der Investitionsförderung und Garantien schwieriger zu kommunizieren (Kredite werden über Treuhandbanken vergeben, und Garantien über die Kommerzbanken vermittelt, wobei den Unternehmen oft nicht bekannt ist, dass die aws dahinter steht), zweitens sind die Themen Schutzrechte und Start-up für einige Unternehmen von geringer Relevanz (hohe Unterschiede der Bewertung je nach Häufigkeit des Kontakts mit der aws), und drittens können die eher heterogenen Bewertungen auch teilweise thematische Überlappungsbereiche der beiden Agenturen ansprechen. Immerhin werden die Themen Schutzrechte, Garantien (im Basisprogramm) sowie Start-up Unterstützung auch von der FFG bedient.

Abbildung 27: $\quad$ Wie klar ist Ihnen, an welche Agentur (FFG oder aws) Sie sich bei den exemplarisch aufgelisteten Anliegen wenden sollen? (FFG KundInnen)

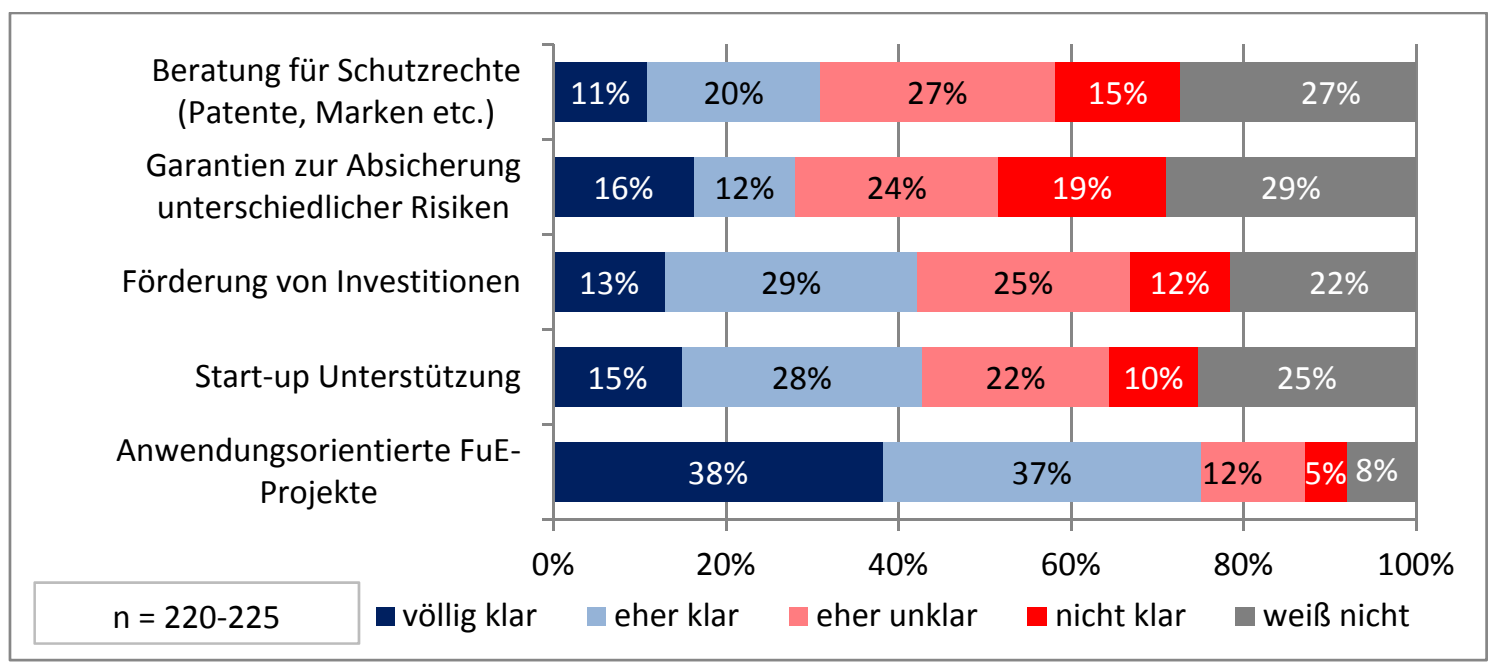


Abbildung 28: $\quad$ Wie klar ist Ihnen, an welche Agentur (FFG oder aws) Sie sich bei den exemplarisch aufgelisteten Anliegen wenden sollen? (aws KundInnen)

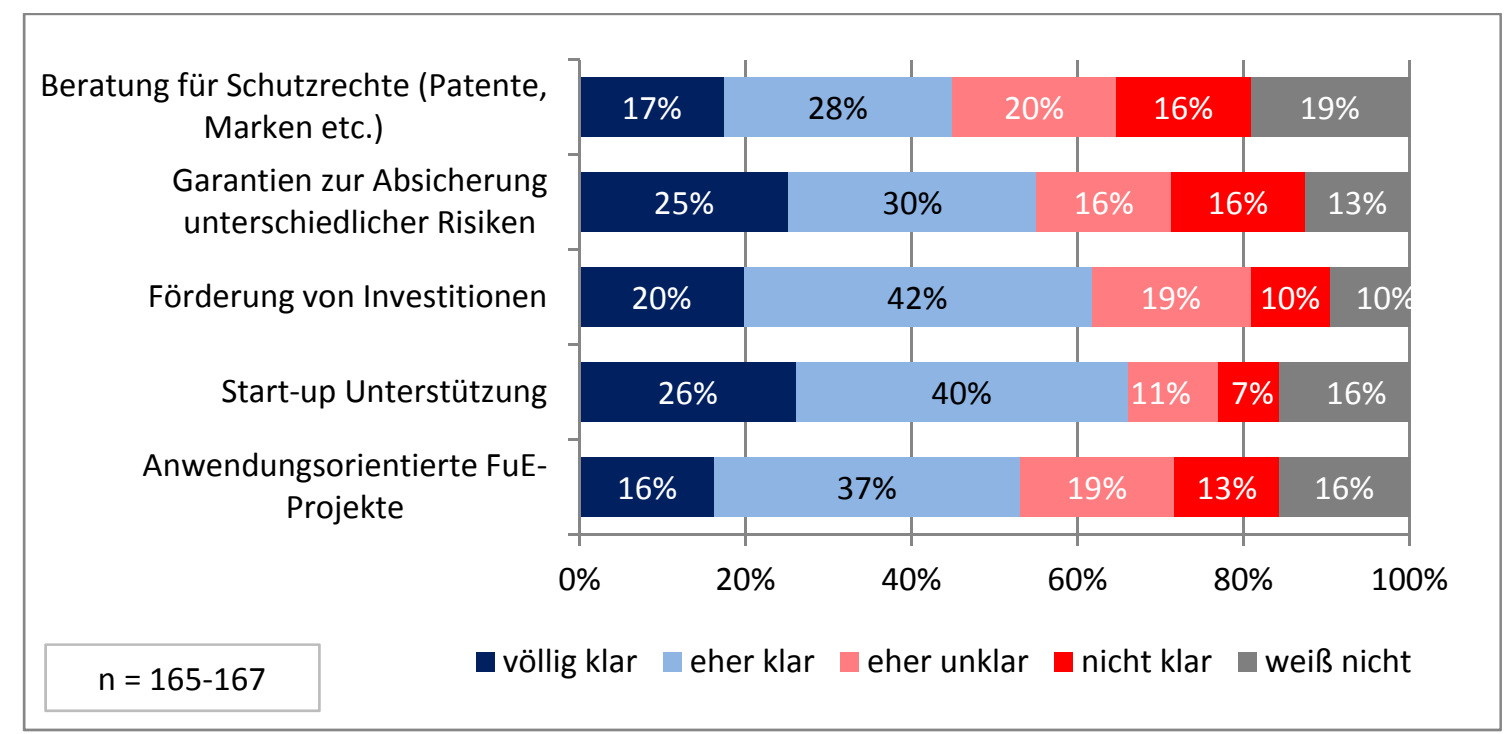

Diese Ergebnisse werden empirisch noch dadurch ergänzt, dass 32\% der Befragten aus dem FFG Survey (41 Unternehmen) meldeten, dass sie innerhalb der letzten fünf Jahre eine aws Förderung in Anspruch genommen hatten, sowie $41 \%$ aus dem aws Survey (65 Unternehmen) meinten, dass sie innerhalb der letzten fünf Jahre eine FFG Förderung in Anspruch genommen hatten.

Eine Schätzung der beiden Agenturen (Kundendaten dürfen derzeit aufgrund von Datenschutz nicht ausgetauscht werden) zeigt, dass jährlich rd. 110 Unternehmen zeitnah gefördert werden. Aufgrund der oben genannten Werte durch die Unternehmen (Ungenauigkeiten sind diesen Angaben natürlich inhärent), dürfte diese Schätzung eher eine Untergrenze darstellen.

\subsection{Koordination}

Ein Koordinationsbedarf zwischen den beiden Agenturen kann mehrere Ursachen aufweisen. (1) Einerseits begründet sich dieser mit einer sukzessiven Unterstützung von Unternehmen, die im Rahmen der FFG Programme FuE-Projekte erfolgreich abgeschlossen haben und im Sinne einer Anschlussfinanzierung einen erhöhten Investitionsbedarf aufweisen. Hier kann z.B. ein Unternehmen aus den FFG Programmen nach einem erfolgreich abgeschlossenen FuE-Projekt um eine Investitionsunterstützung zur Marktüberführung bei der aws ansuchen. Es gibt jedoch auch umgekehrt verlaufende Fälle. So kann etwa ein Unternehmen aus der High-Tech Start-up Förderung nach Abschluss des seed-Programms ein FuE-Projekt bei der FFG verfolgen, oder etablierte 
Unternehmen verfolgen eine FuE-Förderung und eine Investitionsförderung parallel. (2) Andererseits ist eine etwaige parallele Unterstützung durch beide Agenturen für ähnlich gelagerte Projekte von demselben Unternehmen hintanzuhalten, um ungewollte Mehrfachförderungen zu unterbinden. Dies wäre z.B. insofern möglich, als Projekte der experimentellen Entwicklung in der FFG auch ähnlich gelagerte Investitionen wie sie der ERP-Fonds mit seinem nun verstärkten Innovationsschwerpunkt fördert, in Anspruch nehmen könnten. Auch im Bereich High-Tech Gründerförderung könnte dies passieren. (3) Ein weiterer Fall für eine mögliche Koordination wäre z.B. auch ein Austausch der Institutionen hinsichtlich eines gegenseitigen Lernens zu unterschiedlichen Themen, wie etwa im Bereich Prozesse und Strukturen.

\section{Entscheidungskriterien zur Allokation von Förderprogrammen}

Die Trennung zwischen "angewandter Forschung" (FFG) und "Markt" (aws) beinhaltet notwendigerweise einen kleinen Überlappungsbereich, obwohl die meisten InterviewpartnerInnen entlang dieses Kontinuums eine Unterscheidung zwischen den beiden Agenturen sehen. MinisteriumsvertreterInnen bestätigen darüber hinaus, dass die Entscheidungen über Programmbeauftragungen oftmals historisch gewachsen sind und daher Zuordnungen von Programmen zu entweder FFG oder aws von außen nicht immer ganz nachvollziehbar sind bzw. waren. Hier ist insbesondere das Portfolio der vormaligen Innovationsagentur angesprochen, oder die kürzlich vollzogene Verschiebung der akademischen Gründerzentren AplusB von der FFG zur aws.

Die meisten InterviewpartnerInnen stimmten den informellen, aber scheinbar weitgehend gelebten Kriterien zu, auf deren Basis entsprechende Entscheidungen getroffen werden (sollten). Einerseits ist die Zuordnung von Programmen zu einer Agentur abhängig von der Marktnähe bzw. der abgedeckten Teile der Technology Readiness Levels (TRLs), oder anders ausgedrückt: Die FFG ist für industrielle Forschung sowie experimentelle Entwicklung zuständig, und die aws für die Marktüberführung. Das zweite Kriterium entspringt der Frage, ob für die erfolgreiche Bearbeitung eine vorrangige Projektsicht ausreicht (FFG) oder ob das beantragende Unternehmen als Ganzes für die Beurteilung des Förderfalls in den Vordergrund tritt (aws). Der von einem Interviewpartner formulierte und repräsentative Kernsatz lautet: "Die FFG macht Projektförderung und liegt bezüglich der TRLs im mittleren Bereich. Die aws macht Unternehmensförderung und liegt bezüglich der TRLs am marktnahen Ende des Spektrums." Aber auch mit dieser Unterscheidungsmöglichkeit lässt sich ein gewisser Graubereich nicht vermeiden, wie die nachfolgende Diskussion aufzeigt. 


\section{Umgang mit Überlappungsbereichen}

Wie bereits erwähnt, erfolgte die Abstimmung zwischen den Agenturen bislang bilateral auf Ebene der SachbearbeiterInnen und wird seit kurzem noch durch den Quick-Check und den Förderpiloten unterstützt. Die gemeinsam durchgeführten Programme umfassen GIN und Frontrunner, in denen es eine regelmäßige Abstimmung gibt. Weitere, nicht geregelte Schnittstellen bzw. Überlappungen gibt es zu den folgenden Themen:

\section{Gründungsförderung}

Gründungsunternehmen, denen es gelingt, eine ausreichende Eigenkapitaldecke darzustellen (mit Venture Capital, Ausgründungen von größeren Unternehmen, etc.), können z.B. bei der FFG im Basisprogramm bis zu 70\% Unterstützung für ihr FuE-Projekt erhalten. In dem daran anschließenden Programm Markt.Start werden die Bedingungen durch längere tilgungsfreie Zeiten, Überprüfung von Meilensteinen etc. als günstiger eingestuft als bei ähnlichen Programmen in der aws. Dies veranlasst Unternehmen oder Venture Capital VertreterInnen dazu, mit forschungsorientierten Unternehmen, die noch eine Forschungsleistung bis zur Marktreife zu erbringen haben, bei der FFG vorstellig zu werden. Andernfalls ist das PreSeed Programm der aws attraktiver. Wenn Unternehmen bereits vom Seed Programm unterstützt werden, können Sie etwaige Forschungsprojekte gesondert bei der FFG beantragen.

Die aws konnte sich insbesondere in den letzten Jahren erfolgreich mit dem Thema Gründung assoziieren. Im Jahr 2016 ergingen rund 25\% der Gesamtfinanzierungsleistung an Start-ups, mit der Tendenz nach oben (aws-Mehrjahresprogramm 2016). Darüber hinaus ist auch darauf hinzuweisen, dass die FFG im Zeitraum 2009-2014 im Durchschnitt rund 15\% des FFG Budgets in innovative Start-Ups investierte (FFG Mehrjahresprogramm 2015-17). Es gibt hier also durchaus zwei Wege zur Finanzierung der Gründungsphase, abhängig davon, wie forschungsintensiv das Unternehmen bzw. das beantragte Projekt ist, und ob die (zur aws) relativ höheren Anforderungen der FFG für den nachzuweisenden Eigenkapitalanteil dargestellt werden kann. Letzteres bezieht sich darauf, dass die FFG im Gegensatz zur aws keine eigenen Instrumente hat, um das Eigenkapital der Unternehmen anzuheben.

\section{Schutzrechte}

Im Themenfeld Schutzrechtsberatung ist eine Vielzahl an Stakeholdern involviert. Diese reichen von der Wirtschaftskammer über Gründerzentren bis hin zu den Außeninstituten der Universitäten. Die aws arbeitet mit ihren Schutzrechts-Programmen, ebenso wie die FFG mit ihrem Förderprogramm Patentscheck seit 2016, mit dem Patentamt zusammen. Darüber hinaus hat das Patentamt selbst seit 2017 damit begonnen, eigene Produkte 
mit Markennahmen für ihre Schutzrechts-Services zu entwickeln. Daraus lässt sich ein Abstimmungsbedarf ableiten, da eine Schutzrechtsfragestellung in der Regel bereits nahe der Marktüberführung gesehen wird. Die konkrete Argumentationslinie ist hier jedoch nicht eindeutig. Die FFG argumentiert, dass sie den Bedarf für und die Ausgestaltung des Patentschecks aufgrund ihres FFG-eigenen Monitorings entwickelt hat, und dass dieser durch die nun sehr praxisnahe Ausgestaltung auch hohe Nachfrage erfährt. Die aws, in der rund 20 Personen mit dem Thema Schutzrechte befasst sind und eng mit dem Patentamt zusammenarbeitet, sieht hierin hingegen die mangelnde Weiterleitung von Unternehmen mit entsprechenden Bedarfslagen durch die FFG, die von der aws im Rahmen ihrer Programme abgewickelt werden könnten (die Weiterleitung zu anderen Themen wird jedoch als problemlos dargestellt). Hier steht einerseits ein Wettbewerb über Ideen an einzelnen Schnittstellen und andererseits die Frage im Mittelpunkt, inwiefern eine verstärkte Koordination zwischen den Agenturen erfolgen sollte.

\section{Überführung in den Markt}

Mit dem Instrument Markteinführung bzw. dem Programm Markt.Start ist die FFG ebenfalls in einem Bereich tätig, der nach der obigen Abgrenzung von einigen Stakeholdern als eher bei der aws angesiedelt gesehen wird. Die FFG hat dieses Programm eigenen Angaben zufolge gestartet, da sie festgestellt hatte, dass die Kriterien der Marktüberführungsprogramme bei der aws für eine bestimmte Kundengruppe als zu hochschwellig angesehen wurden (in der FFG Variante gibt es eine längere tilgungsfreie Zeit, Überprüfung von Meilensteinen etc.) bzw. dies als eine logische Abrundung des Programmportfolios gesehen wird (als Abschluss eines FuE Projekts). ${ }^{8}$ Interviews und Befragungen von Kundlnnen der FFG und aws im Rahmen der gegenständlichen Evaluierung bestätigen diesen Sachverhalt ("eines der sinnvollsten Programme für Start-ups"). Von Seiten der Prinzipale wurde - beispielhaft anhand des konkreten Falles Markt.Start - hingegen formuliert, dass es nicht Sache der Agentur sei, derartige Probleme einseitig zu lösen. Bei der Identifikation eines Problems sollte die Agentur die potentiellen Auftraggeber adressieren und mit diesen gemeinsam eine Lösung finden. Die Lösung wäre im gegebenen Fall evtl. gewesen, an den Kriterien der aws Programme etwas zu ändern. Eine ähnliche Argumentationslinie kann zum Thema Venture Capital herangezogen werden.

\section{Organisationales Lernen}

Organisationales Lernen voneinander bezüglich Prozessen und Strukturen basiert auf informellem Wege auf der Ebene der SachbearbeiterInnen, bei Abstimmungstreffen, bei

8 MarktStart wird aus den Darlehensrückflüssen des Basisprogramms finanziert und kann nicht beantragt werden, wenn davor eine Seed-Finanzierung der aws bezogen wurde. 
gemeinsamen Auftritten der Agenturen gegenüber den Kundlnnen oder im Zuge der Qualitätssicherung. Auch bei der Nominierung von Jury-Mitgliedern gibt es gegenseitige Hilfestellungen. Ein strukturierter Prozess des gegenseitigen Lernens wurde bislang jedoch nicht aufgesetzt. Der Grund hierfür liegt darin, dass die beiden Organisationen aufgrund der inhärenten Dynamik ihrer Geschäftsfelder eher auf interne Prozesse, auch mit ihren Auftraggebern fokussiert waren, und es darüber hinaus als vielversprechender angesehen wurde, einen strukturierten Lernprozess bei ähnlichen, internationalen Agenturen zu unterhalten. Dies wird von beiden Agenturen mit ihrer aktiven Mitgliedschaft im TAFTIE Netzwerk (FFG) sowie der European Association of Guarantee Institutions (aws) umgesetzt. Auch wenn diese Argumentation durchaus nachvollziehbar ist, fällt doch auf, dass es einzelne Themen gibt, die durchaus von einer gegenseitigen Befruchtung profitieren könnten. In diesem Zusammenhang seien z.B. das organisationsübergreifende Clientmanagement-System der aws oder die strikte personelle Trennung der Prozessschritte Beratung, Begutachtung und Kontrolle in der FFG angeführt.

\section{$6.4 \quad$ Fazit}

Die beiden Agenturen sind mit ihren spezifischen Geschäftsmodellen im nationalen innovationspolitischen Umfeld klar positioniert. Sie fungieren in weiten Teilen als one-stop Shop für anwendungsorientierte FuE (FFG) sowie investiver Unternehmensförderung (aws). Aus diesen unterschiedlichen Blickwinkeln unterstützen beide Agenturen innovative Projekte, wie auch in ihren jeweiligen gesetzlichen Grundlagen definiert. Im Falle der aws ist die Positionierung dahingehend etwas einzuschränken, als es auf nationaler Ebene noch weitere, kleinere Agenturen mit komplementärem Portfolio gibt (ÖHT, ÖKB, ÖEB). Diese Sichtweise ist unbeschadet der Tatsache, dass die relativ hohe Strukturiertheit auf nationaler Ebene auf ein teilweise noch nicht abgestimmtes Portfolio der Bundesländer trifft - wobei es jedoch auch hier positive Ausnahmen gibt. Dies liegt jedoch außerhalb der Entscheidungsmacht des Bundes bzw. der Agenturen.

Eine stärkere horizontale Koordination zwischen den Agenturen im österreichischen Fördersystem wurde durch die Strukturreform 2002-2004 erreicht. Dies trifft jedenfalls auf die nun in Form von Bereichen weitergeführten vormaligen Agenturen zu, die aufgrund der Zusammenführung vielfältige Vorteile ausschöpfen konnten. Auch wenn ein kontrafaktischer Vergleich per se nicht möglich ist, können die beiden Agenturen FFG und aws die betreffenden Themen nun koordinierter bearbeiten, als es in einem Szenario mit acht Organisationen möglich gewesen wäre. Es gibt jedoch noch weiteres Optimierungspotenzial. Zum Beispiel können agenturinterne Prozesses nicht gänzlich vereinheitlicht werden, wenn die Auftraggeber unterschiedliche Bedürfnisse für Dokumentation und Reporting aufweisen, oder unterschiedliche Governance-Strukturen der Eigentümer die 
Möglichkeiten zur Harmonisierung von Organisationsstrukturen hintanhalten (z.B. TP und EIP in FFG, oder ERP in aws). Dieses Optimierungspotenzial kann nur bei größerer Unabhängigkeit der Agenturen ausgeschöpft werden, indem die Agenturen ihre Förderinstrumente selbst definieren und dadurch eine strategische Themensteuerung (durch die Auftraggeber) ermöglicht bzw. erleichtert wird.

Darüber hinaus sollte die Incentive-Struktur für die Agenturen insofern geändert werden, als derzeit von beiden Agenturen eine Förderbudgetmaximierung betrieben wird, da bislang Innovationen im Portfolio aufgrund der Beharrlichkeit von existierenden Programmen fast ausschließlich auf diese Weise möglich waren. Dies hat auch zur Konsequenz, dass es am Rande der eigentlich ausreichend unterschiedlichen Geschäftsmodelle der beiden Agenturen zu Überlappungen aufgrund von Konkurrenzgedanken oder der innerbetrieblichen Sicht zur Abrundung des eigenen Portfolios kommt. Dies wird derzeit dadurch verstärkt, dass zusätzliche Budgets für Themen gerade in diesen Randbereichen zur Verfügung gestellt werden.

Der Überlappungsbereich der beiden Agenturen ist quantitativ betrachtet relativ klein. In dem gemeinsam bearbeiteten Bereich betrifft dies die Programme GIN und Frontrunner, die bereits von Beginn an als gemeinsam abzuwickelnde Initiativen konzipiert wurden. Darüberhinausgehende Überlappungen zwischen FFG und aws liegen in den Bereichen Unternehmensgründung, Schutzrechte, VC und Überführungen in den Markt. Die Abgrenzung zwischen den beiden Agenturen kann dabei insofern noch geschärft werden, indem die Kriterien zur Beauftragung der einzelnen Agenturen expliziter gemacht werden. Damit ließen sich die Überlappungsbereiche klein halten, jedoch nicht völlig beseitigen. ${ }^{9}$ Da eine trennscharfe Abgrenzung nicht gänzlich möglich ist, muss eine gute Zusammenarbeit und Abstimmung an diesen Randbereichen erfolgen, die bereits im Stadium des Designs der Intervention beginnt.

Es ist Aufgabe der Eigentümer/Auftraggeber dafür zu sorgen, dass Themen, die eine Brücke zwischen Unternehmenssicht/FuE/Marktüberführung erfordern, von den Agenturen auch gemeinsam bearbeitet werden, und damit komplementäre Kompetenzen gebündelt werden. Die Auftraggeber sollten in diesem Fall bereits im Designstadium beide Agenturen einbinden, damit informierte Lösungen entwickelt, und eine gute Zusammen-

9 Die konzeptionellen Überschneidungen lassen sich nicht ganz beseitigen, egal welche Kriterien zugrunde gelegt werden. Dies wird noch dadurch verstärkt, dass sich die Bedarfe im Zeitablauf verändern können (z.B. die Umsetzung eines breiteren Begriffs von Innovation, die Umsetzung von systemischen Maßnahmen im Sinne eines "systemic change", um die Grand Challenges in Zukunft systematischer zu bearbeiten). Darüber hinaus könnte bei dem Versuch, eine absolute Überschneidungsfreiheit zu erzielen auch eine von keiner Agentur angesprochene thematische oder Zielgruppen-"Restmenge" entstehen. 
arbeit während der Umsetzung sichergestellt wird. Auch unter der Perspektive einer größeren operativen Unabhängigkeit der Agenturen kann eine Kooperation der Agenturen erwirkt werden, wenn klar kommuniziert wird, welchen Kriterien ein vorgeschlagener Instrumentenmix zu entsprechen hat. Dies bedeutet dann in der Praxis, dass ein Vorschlag der Agenturen nur akzeptiert wird, wenn eine Abstimmung im Vorfeld erfolgte, und im berechtigten Fall ein gemeinsames Paket angeboten wird.

Wenn in Zukunft vermehrt eine Koordination im Vorfeld von politischen Initiativen stattfinden soll, wäre eventuell eine gegenseitige Repräsentanz in den Gremien (z.B. Aufsichtsratsmitglied mit beratender Stimme) überlegenswert, um damit eine Abstimmungsmöglichkeit zu institutionalisieren.

Darüber hinaus ist bezüglich der Abstimmung der gemeinsame Auftritt der Agenturen gegenüber den KundInnen sehr wichtig. Dies wurde bereits in der Vergangenheit so praktiziert, und nun mit dem Förderpiloten und den Quick-Check auch online umgesetzt. Daran gilt es weiterzuarbeiten. 


\section{$7 \quad$ Steuerung und Governance}

Das folgende Kapitel ist der Analyse der Governance bzw. Steuerung der Förderungsagenturen FFG und aws durch die beiden Bundesministerien für Verkehr, Innovation und Technologie (bmvit) und Wissenschaft, Forschung und Wirtschaft (bmwfw) gewidmet, die sowohl die Eigentümerfunktion des Bundes gegenüber den beiden Agenturen ausfüllen, als auch deren zentrale Auftraggeber sind. Es werden die im Rahmen der vorliegenden Evaluierung gewonnenen Erkenntnisse zum aktuellen Verhältnis der Ressorts zu den Agenturen sowie zentrale Entwicklungen aufgezeigt und diskutiert, um letztlich die folgenden Evaluierungsfragen beantworten zu können:

- Welches Selbst-, Fremdbild und Rollenverständnis der FFG bzw. der aws im Fördersystem hat sich entwickelt?

- Welches wechselseitige Rollenverständnis von principal(s) und agent hat sich entwickelt? Kam es zu Gewichtsverlagerungen? Wie scharf ist die Trennung zwischen strategischer und operativer Funktion? Inwieweit sind Befunde, die Steuerungs- und Prinzipal-Agent-Problematik betreffend in den gewählten bzw. gewachsenen Strukturen angelegt?

- Welche Wechselwirkungen ergeben sich zwischen Rollenverständnis etc. und Steuerungsfragen?

- Wie kann die bestehende Vielfalt der Zusammenarbeitsmodi zwischen Ministerien und FFG bzw. aws beurteilt werden? Wie ist das Finanzierungs- und Beauftragungssetting (Einzelverträge, Ausführungsverträge, Rahmenvertrag, autonome Finanzierungsbereiche, heterogene Situation bei der Autonomie der Programmabwicklung) hinsichtlich der entsprechenden Auswirkungen auf Steuerung und Governance zu bewerten?

- Wie gut funktioniert das institutionelle Konstrukt FFG bzw. aws gemäß ihres jeweiligen Auftrags?

- Konnten die mit der Strukturreform verbundenen Ziele der Reduktion organisatorischer und inhaltlicher Komplexität sowie der Lösung immanenter Koordinationsprobleme erreicht werden?

- Welche Verbesserungspotenziale lassen sich mit Blick auf die Ziele der Strukturreform im Bereich der Governancestrukturen zwischen strategischer und operativer Ebene identifizieren?

Dementsprechend ist das Kapitel wie folgt strukturiert: auf eine einleitende Darstellung der Ausgangssituation und des entsprechenden Evaluierungsansatzes folgt die Erläuterung der Zielsetzung dieses Kapitels sowie der entsprechend gewählten methodischen Herangehensweise. Die inhaltliche Diskussion folgt den zentralen Themenschwerpunkten Arbeitsteilung und Rollenverständnis, Steuerungsmechanismen und -kanäle sowie 
Einfluss und Steuerbarkeit. Darin erfolgt jeweils die Analyse des Status quo, der wesentlichen Entwicklungen seit der Schaffung der beiden Agenturen bzw. seit der Systemevaluierung sowie die Diskussion von Wechselbeziehungen zwischen den analysierten Elementen. Abschließend werden die Erkenntnisse vor dem Hintergrund des gewählten Analyseansatzes, der Evaluierungsfragen sowie der vier Strukturreformziele zusammenfassend dargestellt.

\subsection{Ausgangssituation und Evaluierungsansatz}

Ausgangspunkt für die Betrachtung der derzeitigen Governance (sowie seiner Entwicklung) im Verhältnis von Agenturen und Ministerien waren die Strukturen und Mechanismen, die mit dem Forschungsförderungs-Strukturreformgesetz 2004 bzw. dem Austria Wirtschaftsservice-Errichtungsgesetz 2001 geschaffen und seitdem modifiziert wurden (z.B. durch die Zusammenlegung von Wirtschafts- und Wissenschaftsministerium oder die Einführung der wirkungsorientierten Haushaltsführung). Eines der wesentlichen Ziele, das mit diesen Strukturreformen verbunden wurde, war die Reduktion organisatorischer und inhaltlicher Komplexität. Die Vielfalt der Akteure und deren relative Unabhängigkeit von politischen Strategieprozessen wurde vor deren Zusammenlegung als Steuerungserschwernis und mitunter -verlust der Politik interpretiert. Durch die Schaffung von Förderungsgesellschaften in Form von Agenturen sollten daher immanente Steuerungs- und Koordinationsprobleme gelöst werden. Die Tatsache, dass es sich bei den neu geschaffenen Organisationen um ressortübergreifende Agenturen handelte, war gleichzeitig ein potenzielles Hindernis für eine effektive Steuerung.

Das Governance-Verhältnis der beiden Förderungsagenturen FFG und aws zu den beiden Eigentümerministerien war und ist im österreichischen FTI- und Wirtschaftsförderungs-System insbesondere durch jene Prozesse bestimmt, die sich unter dem Begriff "Agencification" subsumieren lassen. Daraus folgt eine Reihe von Herausforderungen an das System, das zudem durch historische Entwicklungen und nationale Eigenheiten geprägt ist. Die mit dem Forschungsförderungs-Strukturreformgesetz von 2004 eingeleitete adaptierte Agencification sollte in erster Linie für verbesserte Steuerungsfähigkeit der Regierung sowie eine professionellere und flexiblere Verwaltung unter der Maßgabe erhöhter Effizienz stehen (vgl. Pichler, Stampfer, Hofer 2007). Der gesamte Prozess kann als unvollständige Agencification (vgl. Pichler 2016) betrachtet werden, da die dahinterliegende Logik der Auslagerung von Aufgaben (im vorliegenden Fall z.B. der in den Bundesministerien noch verbliebenen Ressortforschung) in Agenturen intakt blieb, wenngleich die üblicherweise damit verbundene und zu beobachtende Ausdifferenzierung der Agenturlandschaft nicht gegeben war. Vielmehr wurden bestehende Agenturen 
fusioniert, um eine bessere, effektivere Steuerung durch die Politik (wieder) zu ermöglichen. Über diese vom Idealtypus der Agencification abweichende Vorgehensweise hinaus, blieben allerdings auch weitere Besonderheiten erhalten, wie etwa das Prinzip der Eigenmittel der FFG (entsprechend der Fondsstruktur des Vorgängers FFF) oder die geteilte Eigentümerschaft und Finanzierung der Agenturen durch mehrere Bundesministerien auf dem Wege der Beauftragung.

In direktem Zusammenhang damit steht außerdem die Prinzipal-Agent Theorie als analytisches Rahmenkonzept zur Verfügung. Ursprünglich in den Wirtschaftswissenschaften als Teil des Modells der Neuen Institutionenökonomik entwickelt, ist dieses Konzept inzwischen auch in anderen Disziplinen und insbesondere in der Politikwissenschaft etabliert. Vor dem Hintergrund von Erkenntnissen der Bürokratieforschung zum Verhältnis von politischem System und (vor allem) der Ministerialbürokratie, d.h. des Phänomens des so genannten bureaucratic drift, also der Verselbstständigung der staatlichen Bürokratie gegenüber dem direkten Mandat ihrer Auftraggeber, hat dieser Ansatz es ermöglicht, entsprechende empirische Phänomene fassbar zu machen. Im Wesentlichen hat die Beobachtung, dass bereits im Rahmen des Verhältnisses von Politik und Verwaltung rein hierarchische Steuerungsinstrumente versagen (können), dazu geführt, dass ein anderes Verständnis von an komplexen Prozessen innerhalb des politischen Systems beteiligten Akteuren notwendig wurde (vgl. Downs 1967; Niskanen 1971; Braun und Gilardi 2002). Verstärkt durch die auf dem Ansatz des New Public Management basierenden Auslagerungen von Aufgaben aus der Verwaltung in rechtlich eigenständige Organisationen (Agenturen) hat die Orientierung an den Begriffen und Untersuchungsdesigns der Prinzipal-Agent Theorie eine neue Analyseperspektive ermöglicht.

Wichtig ist dabei, dass eine Prinzipal-Agent Beziehung dann vorliegt, wenn es eine gewisse Abhängigkeit in Bezug auf das "Wohlergehen" einer Partei (Prinzipal) von den Handlungen einer anderen Partei (Agent) gibt (vgl. Pratt und Zeckhäuser 1985). In aller Regel gibt es in diesen Prinzipal-Agent Beziehungen einen Auftraggeber (Prinzipal), der einen Auftragnehmer (Agent) mit einer Aufgabe betraut. Diese Beauftragung erfolgt in der Annahme, dass der Agent die Aufgabe im Sinne des Prinzipals erfüllt. Im Zentrum steht dabei die Annahme, dass Organisationen (Prinzipale, wie im vorliegenden Fall Ministerien) in ihrer Entscheidungsfindung (und damit auch ihren Möglichkeiten zur effektiven Steuerung anderer Organisationen - Agenturen) eingeschränkt sind, z.B. durch asymmetrische Informationsverteilung. Prinzipale verfügen demnach nur über unvollständige Informationen, auf deren Basis sie das Handeln ihrer Agenten beurteilen sollen. Demgegenüber haben Agenten einen Informationsvorteil, da nur sie das eigene Verhalten (in unterschiedlichem Ausmaß) direkt selbst festlegen und entsprechend beurteilen 
können. In solchen Beziehungen kann es dazu kommen, dass die Informationsasymmetrie zu Ungunsten des Prinzipals ausgenutzt wird, wenn dies aus der Sicht des Agenten sinnvoll erscheint (Stichwort: moral hazard).

Mit anderen Worten, die Prinzipal-Agent Theorie ermöglicht einen kritischen Blick auf die anderenfalls als überlegen und effizienter wahrgenommene Tendenz zur Agencification (im Unterschied zum klassischen Verbleib von Aufgaben im politischen System und vor allem in der Verwaltung; vgl. Pichler 2014). Durch diesen Ansatz wird der Blick frei auf die möglicherweise nicht unbedeutenden Kosten aufgrund des erhöhten Steuerungsund Informationsaufwandes seitens des Prinzipals und andere Probleme. Gleichzeitig bietet der Ansatz einige Mechanismen, wie die Gesamtproblematik im Verhältnis Prinzipal zu Agent gelöst werden kann, z.B. durch die (Wieder-)Einführung von hierarchischer Kontrolle, Controlling- und Anreizsystemen, Vertrauen, Erfolgskontrollen, usw. (vgl. Braun 1993; 1998; Braun und Guston 2003; Guston 1996). Daher bietet dieser Ansatz nicht nur einen passenden analytischen Rahmen für die Identifikation bestehender Probleme in der Governance zwischen Agenturen und Ministerien, sondern darüber hinaus auch Elemente zur Lösung entsprechender Probleme, die sich theoretisch und zumindest in Ansätzen in einer empirischen Überprüfung identifizieren und bewerten lassen sollten (vgl. Lane 2013). Insbesondere bietet dieser Ansatz den Rahmen, zu überprüfen, inwieweit in den letzten Jahren eine schärfere Trennung zwischen strategischer und operativer Funktion zwischen principal(s) und agent herbeigeführt werden konnte und welchen Einfluss dies auf das eigene sowie das gegenseitige Rollenverständnis der beteiligten Akteure hat.

\subsection{Zielsetzung und methodische Vorgehensweise}

Die vorliegende Evaluierung fokussierte in diesem Zusammenhang auf die Frage nach dem Status quo und der Entwicklung der Steuerung und Governance (sowie der entsprechend genutzten Strukturen und Mechanismen) der beiden Förderungsagenturen FFG und aws durch die beiden Eigentümerministerien bmvit und bmwfw. In diesem Zusammenhang wurden Einflussvariablen und Kontextinformationen erhoben und auf ihre Wirkung auf die Governance überprüft. Dazu zählten organisatorische Charakteristika, (eigene und gegenseitige) Wahrnehmungen, das wechselseitige Rollenverständnis usw. unter Berücksichtigung der verschiedenen Modi der Zusammenarbeit (z.B. im beauftragten vs. autonomen Bereich, Eigentümer- vs. Auftraggeberfunktion), Freiheitsgrade bei der Umsetzung von Programmen (etwa durch die Nutzung verschiedener Finanzierungs- und Beauftragungssettings oder aufgrund unterschiedlicher Auffassungen zur notwendigen politischen Interventionstiefe seitens der Ministerien). Zusätzlich zur Frage nach der Steu- 
erbarkeit der Agenturen war auch die Frage nach der Steuerungsfähigkeit seitens der Bundesministerien inklusive der entsprechenden Einflussfaktoren relevant. Im Zentrum stand die Kernfrage, inwieweit die mit der Errichtung der FFG und der aws verbundenen Ziele der Verbesserung der Steuerungsfähigkeit (durch u.a. die Reduktion organisatorischer und inhaltlicher Komplexität) erreicht werden konnten.

Den Ausgangspunkt für die Analysen im Rahmen dieses Arbeitspaketes bildete eine umfangreiche Sichtung der rechtlichen und administrativen Unterlagen (relevante Verträge, Strategie- und Programmdokumente, Leitbilder, Richtlinien, aws-Gesetz, FFGGesetz, Rechnungshofberichte etc.) sowie eine Auswertung der einschlägigen Studien und Evaluierungen (z.B. Systemevaluierung, Forschungs- und Technologieberichte etc.). Darüber hinaus wurde zur theoretischen Fundierung der Ergebnisse die aktuelle wissenschaftliche Literatur zu den entsprechenden Themen Governance (insbesondere in Mehrebenensystemen), Agencification, New Public Management etc. analysiert.

Um die Entwicklung der Governance seit der Gründung der Agenturen nachzuvollziehen, wurden zunächst 25 narrative Interviews 10 mit VertreterInnen der Agenturen und Ministerien (bzw. weiteren externen ExpertInnen des FTI- und wirtschaftspolitischen Systems mit entsprechendem Überblickswissen, wie z.B. externe EvaluatorInnen) durchgeführt. Diese dienten dazu, eine konsolidierte Narration inklusive zentraler Entwicklungsschritte und -ereignisse zu etablieren. Darauf aufbauend wurden diese Entwicklungsschritte, Ereignisse sowie als zentral benannte Themen in 29 Leitfaden gestützten Interviews und zwei Gruppeninterviews (mit insgesamt acht TeilnehmerInnen) ebenfalls mit VerteterInnen der Ministerien und Agenturen vertiefend diskutiert. Zur Abbildung der wechselseitigen Rollenverständnisse und der entsprechenden Zusammenarbeitsmodi zwischen den Ministerien und den beiden Förderagenturen sowie für eine mögliche Differenzierung innerhalb der Agenturen und Ministerien (nach strukturellen Einheiten, eingesetzten Finanzierungssettings und dominanten Zusammenarbeitsmodi) wurde darüber hinaus eine standardisierte Online-Befragung11 der für die Entwicklung und Implementierung relevanten Akteure innerhalb der vier Institutionen (FFG, aws, bmwfw und bmvit) durchgeführt.

10 Für Details bezüglich aller Erhebungsinstrumente siehe Kapitel 2.

11 Die entsprechende Anzahl verwerteter Antworten im Rahmen der Online-Befragung verteilen sich wie folgt auf die einzelnen Organisationen: $F F G=172$, aws $=154$, bmvit und bmwfw $=51$. 


\subsection{Arbeitsteilung und Rollenverständnis}

Die Frage nach der grundsätzlichen und gelebten Arbeitsteilung zwischen Agenturen und Ressorts sowie dem damit verbundenen bzw. dahinterliegenden Rollenverständnis ist von zentraler Bedeutung für die vorliegende Evaluierung. Hier liegt ein wesentlicher Kulminationspunkt der komplexen (Wechsel-)Beziehung von Rahmenbedingungen (in organisatorischer und regulatorischer Hinsicht) und der im Tagesgeschäft dominierenden Interpretation und Implementierung eben dieser Bedingungen.

Grundsätzlich unterscheiden sich die Einschätzungen bezüglich der Rollen und Aufgaben, die üblicherweise von Agenturen und Ministerien eingenommen und übernommen werden, leicht bis teilweise deutlich je nach Organisation, Abteilung, inhaltlich-thematischem Fokus und auch Person. Darin spiegeln sich nicht nur unterschiedliche Sichtweisen, sondern auch die verschiedenen Rahmenbedingungen wider. Es gibt keine einheitliche Arbeitsteilung zwischen Ressorts und Agenturen aufgrund der unterschiedlichen Funktionen, die die Organisationen in den verschiedenen Zusammenhängen einnehmen. Das diversifizierte Rollenverständnis leitet sich auch aus der Doppelfunktion der Ressorts als Eigentümer und Auftraggeber der Agenturen ab, die zumindest im bmvit auch organisatorisch voneinander getrennt sind, d.h. je nachdem in welcher Funktion die Ressorts (überwiegend) auftreten, verändern sich auch die Aufgabenteilung und wahrnehmung.

Die Arbeitsteilung zwischen der FFG und den beiden Eigentümeressorts wird demnach jeweils relativ ähnlich eingeschätzt: Die stärkste Rolle spielen die Ministerien in der Einschätzung der befragten MitarbeiterInnen jeweils bei der Formulierung der übergeordneten Strategien. Aber auch bei der Operationalisierung und Umsetzung neuer Politikkonzepte wird innen eine starke Rolle zugeschrieben. Als klare Aufgabe der FFG wird demgegenüber die Organisation der Projektauswahlverfahren, die Besetzung der Jurys, die Organisation von Begleitveranstaltungen sowie die Erarbeitung der Programmdokumente betrachtet. Von den Ministerien wird die Arbeitsteilung mit der FFG als stärker distinkt wahrgenommen. Außerdem schreiben sich die Ministerien in deutlich höherem Umfang als idealtypisch vorgesehen ein Engagement bei Begleitveranstaltungen, der Vermarktung der Förderprogramme und der Jury-Besetzung zu.

Die Arbeitsteilung zwischen den Ressorts und der aws ist im Prinzip damit vergleichbar, wenngleich es hier eine Verschiebung in Richtung Agentur zu geben scheint, d.h. vor allem in der Wahrnehmung der Ressorts hat die aws im Durchschnitt die Verantwortung für mehr Aufgaben, insbesondere bei der Projektauswahl und Organisation von Begleitveranstaltungen. Die Unterschiede bzw. die Abweichungen von der theoretisch anzunehmenden Arbeitsteilung lassen sich allerdings in Summe nicht ohne weiteres auf die 
aktive Ausweitung der Verantwortlichkeiten oder den ebenso aktiven Rückzug einer der Organisationen aus bestimmten Aufgabenbereichen gleichsetzen. Viele Aufgaben sind hinsichtlich der Verantwortlichkeit nicht klar geregelt (z.B. die Entwicklung strategischer Intelligenz, die Wirkungskontrolle oder die Zielgruppenmobilisierung) und geben so von vornherein Raum für Interpretation und unterschiedliche Handhabung.

Insgesamt unterscheiden sich allerdings die Einschätzungen, die im Rahmen der MitarbeiterInnenbefragung gewonnen wurden, von den Interviewergebnissen. Während in den Interviews (überwiegend mit erfahrenen Personen in höheren Hierarchieebenen) vielfach von einer sehr starken Rolle und teilweise sogar Dominanz der Agenturen über viele Aufgaben ausgegangen wird, zeichnet die durchgeführte Befragung der Ressortund AgenturmitarbeiterInnen ein sehr viel näher an der idealtypisch anzunehmenden Arbeitsteilung liegendes Bild. Hier sind nur wenige Differenzen in der Selbst- und Fremdwahrnehmung zu finden, die ganz überwiegend auf unterschiedlichen Settings basieren (bzw. auf die durchschnittliche Betrachtung der Werte einer je nach Bereich und Setting stark unterschiedlichen Rücklaufquote12).

Was insgesamt deutlich wird, ist, dass es einige Aufgabenbereiche gibt, in denen die Agenturen deutlich aktiver sind als es theoretisch der Fall sein sollte, etwa im Bereich der Entwicklung von Ideen und Strategien und der Erarbeitung der Mehrjahresprogramme. Hier sind die Agenturen in der Selbst- und Fremdwahrnehmung zum Teil für mehr Aufgaben zuständig als die Ressorts, die etwa die Mehrjahresprogramme zwar abnehmen, aber nicht federführend an deren Entwicklung beteiligt sind. Die Erarbeitung der Mehrjahresprogramme ist allerdings auch in den entsprechenden Gesetzen klar als Agenturaufgabe definiert, beantwortet aber nicht unbedingt die Frage, ob dies in letzter Konsequenz sinnvoll sein kann. Darüber hinaus wird die Erarbeitung von Programmdokumenten zum Teil völlig oder überwiegend durch die Agenturen übernommen, was ebenfalls für Leitfäden gilt sowie die in Teilen von den Agenturen ohne Kooperation mit den Ressorts erarbeitete strategische Intelligenz. Auch Programmevaluierungen werden teilweise, vor allem in der aws, direkt beauftragt und nicht regelmäßig veröffentlicht noch offiziell durch die Beteiligung des Auftraggebers 13 des entsprechenden Programms bzw. der Eigentümer begleitet. Dennoch ist es von zentraler Bedeutung festzuhalten, dass unter der Vorgabe entsprechend intensiv kooperierender Gegenüber (d.h. auf ganz indi-

12 Dabei spielt sicher auch der Umstand eine zentrale Rolle, dass zumindest einige der befragten MitarbeiterInnen keinen Einblick in die Entwicklung des Verhältnisses zwischen den Organisationen haben (können).

13 Dabei ist zu beachten, dass die aws für einige ihrer Unterstützungsprogramme Mittel etwa aus der Nationalstiftung erhält, es also streng genommen kein Ressort als Auftraggeber gibt. 
vidueller Basis) in den Ressorts und Agenturen daraus keine echten Probleme entstehen, sondern vielmehr die augenscheinliche Flexibilität in der Handhabung der wenigen, zumeist impliziten Regeln eine durchaus geeignete Grundlage für eine sinnvolle Arbeitsteilung zu sein scheint.

Analog zur starken Rolle der Agenturen gibt es auch den häufig geäußerten Vorwurf gegenüber den Ressorts, Mikromanagement zu betreiben, d.h. bis in kleinste Details der Programmabwicklung hinein Vorgaben zu erteilen und deren Umsetzung zu kontrollieren. Darüber hinaus bestehen Hinweise auf eine deutlich unterschiedliche Handhabung der ressortseitigen Rechte zur Kontrolle etc. durch die einzelnen MitarbeiterInnen und Abteilungen von einer Prüfung der entsprechend festgelegten Wirkungsindikatoren bis hin zu einer detaillierten Prüfung aller eingereichten Förderanträge. Der Kritik an der exzessiven Nutzung formell eingeräumter Prüfrechte seitens der Ressorts stehen allerdings entsprechende Äußerungen gegenüber, die eine genaue Kenntnis der geförderten Projekte für eine wesentliche Basis einer guten strategischen Steuerung halten.

Der Befund, dass die Agenturen über weit mehr Verantwortung verfügen, als zu erwarten wäre, wird von vielen insofern geteilt, als dass für einige Bereiche eher zu gelten scheint, dass die Agenturen in bestimmten Fällen überwiegend oder zumindest zu einem signifikanten Anteil auch die Definition strategischer Ziele und Vorgaben übernehmen oder treiben. Gleiches gilt für die Entwicklung von Programmen bzw. Instrumenten und die Erarbeitung der Programmdokumente. Allerdings gibt es durchaus auch (Themen-)Bereiche, in denen die Ressorts entweder in einem auch aus ihrer Sicht sinnvollen Wechselspiel mit der Agentur agieren oder sogar stark federführend sind. Insbesondere die Frage nach Themenführerschaft und wer letztlich die notwendigen Impulse für die Entwicklung von Programmen etc. setzt, ist damit nicht einheitlich zu beantworten und es existieren in einigen wenigen Fällen sogar direkt konträre Ansichten zwischen Agenturen und Ressorts zur Realität ihrer Zusammenarbeit.

Auch der häufig geäußerte Vorwurf des Mikromanagements seitens der Ressorts in ihrer Eigenschaft als Auftraggeber ist letztlich kein eindeutiger Befund. Es gibt neben einigen sehr auffälligen, teilweise anekdotischen Hinweisen auf ein entsprechendes Verhalten auch Evidenz dafür, dass dies keineswegs als Regel angesehen werden kann. In beiden Agenturen und ihren Eigentümerressorts gibt es auch Erfahrungen einer vertrauensvollen Kooperation, die dem Ressort die strategische Leitung und Konzeption erlaubt, auf einem gemeinsamen Verständnis der für die Umsetzung notwendigen Arbeitsteilung basiert und somit das Führen an der "langen Leine" zum Prinzip macht. Für nahezu alle diese Befunde gilt allerdings eine starke Abhängigkeit von den handelnden Personen, die in ihrem Verhalten zumeist die jeweiligen Rahmenbedingungen reflektieren. 
Hinsichtlich dieser Rahmenbedingungen scheint am ehesten die Frage nach der (potenziellen) Missionsorientierung und der damit verbundenen Finanzierungsbedingungen einer Maßnahme eine Erklärung für die weiter gehende Involvierung der Ressorts in die Umsetzungsarbeit der Agenturen zu geben. Insofern also aufgrund individueller Interessen oder dem Wunsch nach sichtbarer politischer Wirksamkeit MitarbeiterInnen von Ressorts auch auf persönlicher Ebene eine ideelle Eigentümerschaft über ein Programm o.ä. erkennen lassen, verschiebt sich die Arbeitsteilung deutlich in Richtung Ressort. Dabei sind die entsprechenden Rahmenbedingungen naturgemäß gleichermaßen Ausdruck und Folge dessen, d.h. missionsorientierte Unterstützungsleistungen sind viel eher Instrument des sich stetig ändernden politischen Systems und seiner Willensbildung als andere Maßnahmen und legen dementsprechend mehr Involvierung der Ministerialverwaltung (oder sogar direkt der politischen Ebene der Ministerien) nahe. Die Implementierung derartiger Unterstützungsleistungen erfolgt üblicherweise auf dem Wege der Beauftragung; die Kontrolle der finanziellen Ausstattung verbleibt als zentraler Steuerungsmechanismus in der Hand der Ressorts. In Kombination führt dies zu einer wesentlich passiveren Rolle der Agenturen, die sich dann auch eher als ausgelagerte Ministeriumsabteilung ohne eigene Gestaltungsmöglichkeiten wahrnehmen. Aus der Perspektive der Ministerien kommt hinzu, dass insbesondere von der FFG die Aufgaben in diesen Programmen aber ohnehin nicht ausreichend wahrgenommen werden und die Fachabteilungen diese Aufgaben entweder selbst übernehmen müssen bzw. teilweise an Dritte auslagern.

Nicht nur, aber vor allem hier kommt es in einigen Fällen zu einer wechselseitigen Verstärkung dieser vom Idealtypus abweichenden Arbeitsteilung, d.h. die Missionsorientierung der Maßnahme erzeugt eine bestimmte, eher auf eine starke ressortseitige Auftraggeberrolle hin definierte Arbeitsteilung mit entsprechendem Finanzierungsrahmen, was wiederum die Agenturen stärker in die reine Abwicklerrolle drängt (d.h. der Fokus liegt auf der qualitativ hochwertigen, nachvollziehbaren, transparenten usw. Ausführung relativ eindeutig definierter Aufgaben) und darüber hinaus eine entsprechende Wahrnehmung der Arbeitsteilung in den Agenturen erzeugt, die deren Rolle eben auf die Abwicklung reduziert. Aus dieser vergleichsweise passiven Funktion heraus, scheint die Fähigkeit, jedenfalls aber die Möglichkeit der Agenturen zu leiden, eine aktive Mitgestaltung anzubieten. Ressortseitig führt dies teilweise zu der Einschätzung, die Agenturen fielen als kompetente Partner eines inhaltlichen Dialogs aus oder wären außer Stande, bestimmte Aufgaben zu ihrer Zufriedenheit zu übernehmen. Dies führt wiederum zu einer (noch) stärkeren Involvierung der Ressorts oder aber zu der teilweisen Auslagerung von Aufgaben an eine andere Organisation. Das kann im Falle der Entwicklung einer neuen missionsorientierten Maßnahme darin resultieren, dass bestimmte Aufgaben von vornherein nicht an die Agentur übergeben werden. 
In diesem Zusammenhang ist auch relevant, dass ein Teil der beschriebenen Arbeitsteilung darin begründet scheint, dass die entsprechenden BeamtInnen in den Fachabteilungen der Ressorts teilweise an Einfluss und Steuerungsmöglichkeiten an die politischen Ministerkabinette verlieren bzw. aufgrund einer enger gewordenen, wechselseitigen Beziehung zwischen den Agenturen und ihren Leitungsebenen einerseits und den MitarbeiterInnen der Kabinette andererseits weniger an Entscheidungen und Kommunikation partizipieren, worauf sie nach Einschätzung vieler Akteure mit stärkerem Einfluss auf die Umsetzung von Programmen etc. (also Mikromanagement) reagieren. Letztlich drückt sich aber auch hier der Befund einer unklaren und unvollständigen Delegation der Umsetzungsaufgaben an die Agenturen in besonderer Weise (und teilweise eben auch selbstverstärkend) aus d.h. die systemischen Voraussetzungen ermöglichen nicht nur solche Strategien, sondern begünstigen sie auch.

Die unvollständige Delegation und ihre Folgen bzw. die Bedingungen, unter denen sich die Ressorts teilweise in der Richtigkeit einer nicht vollständigen Aufgabendelegierung bestätigt sehen (könnten), werden teilweise durch die Rolle des Bundesministeriums für Finanzen (BMF) verschärft. Dies bezieht sich auf den Umstand, dass obwohl etwa Programme aus dem Haushalt eines Ministeriums finanziert werden, das BMF der eigentlichen Verwendung der Mittel (erneut) zustimmen muss. Als deutlicher Ausdruck dieser komplexen Verflechtung, die zumindest für einige Akteure schwerer wiegt als die Frage nach der Zusammenarbeit zwischen den Agenturen und den Auftraggebern, kann etwa die Konstruktion der Garantieprogramme der aws gesehen werden. Hier verbleibt das Ausfallsrisiko (Schadloshaltung) beim BMF, während BMF und bmwfw jeweils für Garantien nach zwei unterschiedlichen gesetzlichen Grundlagen als Auftraggeber fungieren. Zusätzlich ist das bmwfw als Eigentümer involviert; die Genehmigung muss aber immer auch durch das BMF erfolgen (als Formalprüfung bzw. durch die Vertretung der Eigentümerministerien durch RepräsentantInnen des BMF14 im Aufsichtsrat der aws). Dass ein solches Konstrukt auf individueller Ebene durchaus unterschiedlich interpretiert wird, ist in logischer Konsequenz unmittelbar Folge eines Systems mit unklarer Arbeitsteilung und stark unterschiedlichen institutionellen und rechtlichen Rahmenbedingungen.

Entsprechend der Häufigkeit und Regelmäßigkeit der Zusammenarbeit zwischen den Ressorts und Agenturen bzw. letztlich des relativen Gewichts der einzelnen Ressorts als Auftraggeber vis-à-vis den Agenturen ergeben sich unterschiedliche (und unterschiedlich belastbare) Erkenntnisse für die FFG und aws einerseits und das bmwfw und bmvit andererseits. Insgesamt ist auf Basis der durchgeführten Erhebungen zu erkennen, dass jede Agentur das der Zusammenarbeit zugrundeliegende Rollenverständnis vor allem jenes

14 Das BMF entsendet über bmvit und bmwfw direkt Vertreter in die Eigentümergremien. 
Ressorts kritisiert, mit dem die MitarbeiterInnen die meisten Kontakte haben. Allerdings ist auch erkennbar, dass dem bmvit überwiegend eine vertrauensbasierte Zurückhaltung unterstellt wird, die sich positiv auf die Arbeitsteilung und die Freiheitsgrade der Agenturen bei der Umsetzung auswirkt, obgleich es hier insbesondere in den missionsorientierten Programmen auch zu dem oben diskutierten Mikromanagement kommt. Insbesondere aus der aws (aber auch der FFG, wobei diese naturgemäß weniger mit dem bmwfw kooperiert), gibt es demgegenüber eine kritischere Bewertung des bmwfw sowie vor allem Hinweise auf eine Zunahme der Kontrollversuche durch das Ressort, die sich in zunehmendem Mikromanagement ausdrücken.

Im Vergleich zu den Befunden der Systemevaluierung scheint die Arbeitsteilung zwischen Ministerien und Agenturen eher weniger klar zu sein, wobei der Befund der stark individuellen Auslegung der Regeln etc. bestehen bleibt. In dem von der KMU Forschung Austria verfassten Teilbericht 3 "Governance in der FTI-Politik im Wechselspiel zwischen Ministerien und Agenturen" der Systemevaluierung der österreichischen Forschungsförderung und -finanzierung (2009) hieß es dazu: "Die Arbeitsteilung zwischen den Agenturen und Ministerien ist rechtlich klar, im Grunde wird die Zuordnung von Zuständigkeiten und Umsetzungsaufgaben in der Regel von den meisten Akteuren als eindeutig gesehen. Was hingegen Agenda-Setting und Strategieentwicklung angeht, haben die Agenturen weitergehenden Einfluss, als ihnen anhand der theoretischen Anforderungen an Agenturen zuzuschreiben wäre. Umgekehrt sind in der Wahrnehmung der befragten Akteure Ressorts vielfach auch dort tätig, wo es sich um Aufgaben der Umsetzung handelt."

Insgesamt scheint sich zumindest das Selbstverständnis der Agenturen über ihre eigene Rolle insgesamt und damit ihre "Zugehörigkeit" zum politischen System und der Ministerialverwaltung seitdem gewandelt zu haben. Beide Agenturen betonen ihre Funktion als Dienstleister gegenüber den Fördernehmern zunehmend stärker und insbesondere im Fall der aws ist festzustellen, dass sich die Organisation als Ganzes bewusst nicht als Experten- sondern als Dienstleitungsorganisation (gegenüber den KundInnen, nicht den Eigentümern und Auftraggebern) sieht. Die Agenturen verstehen sich in diesem Sinne nicht (länger) als öffentliche Einrichtungen oder Teil der Verwaltung.

Daraus entsteht ein schwindendes organisatorisches aber auch individuelles Verständnis für Verwaltungs- und juristisch notwendige Prozesse, die in der externen Perspektive einem eher an (nach Auffassung einiger Akteure zu stark betonter) Effizienz und Schnelligkeit ausgerichteten Verständnis widersprechen. Damit einhergehend wird von den Ressorts teilweise beklagt, dass die Agenturen ihre Dienstleistungen vis-à-vis den Fördernehmern zunehmend als eigene Leistungen kommunizieren und die Ressorts damit für die KundInnen zunehmend unsichtbar bleiben. Auch vor diesem Hintergrund sind die 
agenturseitigen Einschätzungen, dass sich die Ressorts zu tief in die operative Umsetzung einbringen und darüber hinaus schnelle Entscheidungen verhindern, möglicherweise als eine - allerdings im Wesen der Agencification angelegte - Auseinanderentwicklung zu bewerten, die mehr als nur eine organisatorische Trennung der zugrundeliegenden Aufgabengebiete bedeutet.

\subsection{Steuerungsmechanismen und -kanäle}

Die Frage nach den Steuerungsmechanismen und -kanälen im Verhältnis der Ministerien zu ihren Agenturen beschreibt die Realität der Entwicklung und Umsetzung von - in erster Linie - Unterstützungsmaßnahmen. Die entsprechenden Befunde sind einerseits direkter Ausdruck der oben diskutierten Arbeitsteilung (und des dahinterliegenden Rollenverständnisses) und geben andererseits Einblick in die grundlegende Verfasstheit des Systems, ausgedrückt in den Formen der Zusammenarbeit der beteiligten Akteure. Damit liegt auch hierin eine zentrale Kristallisation der Einstellungen und Zugangsweise von individuellen und institutionellen Akteuren vor.

Die Zusammenarbeit zwischen den Eigentümerressorts und den Agenturen wird über eine Reihe von unterschiedlichen Vereinbarungen und Verträgen (bzw. Vertragsarten) geregelt (siehe folgende Abbildung). Die Eigentümerfunktion nutzt neben der Entwicklung der Rahmenbedingungen, etwa über Richtlinien oder Abnahme (bzw. über die Aufsichtsräte den Beschluss) der Mehrjahresprogramme (und Arbeitsprogramme im Fall der FFG) inzwischen auch eine explizite Eigentümerstrategie, die von beiden Eigentümern gemeinsam definiert wurde und ihre Herangehensweise an die Governance bzw. Steuerung der Agenturen festhält. Die wesentlichen Instrumente der Eigentümerfunktion (die ja neben der Funktion als Auftraggeber steht), sind darin mit Verweis auf die gesetzlichen Grundlagen aufgelistet: "... auf oberster Ebene die Leitbilder des bmwfw und des bmvit, gefolgt von den Wirkungszielen der beiden Ressorts. Sie bilden aus Sicht der Eigentümer den Rahmen für die Unternehmensstrategie und in weiterer Folge für die operative Umsetzungsverantwortung der Geschäftsführung [...] Die Gesellschaft hat den Eigentümern [...] einen Vorschlag für ein mehrjähriges Programm [...] sowie jährliche Arbeitsprogramme 15 zur Genehmigung vorzulegen." Ebenfalls in der Eigentümerstrategie enthalten sind die Wirkungsziele und Kennzahlen. Die Entwicklung der Kennzahlen und Meilensteine wird regelmäßig im Rahmen der Eigentümer Jour Fixe überprüft. Dazu kommen als Ausdruck der Auftraggeberfunktion entsprechende Ausführungsvertrage bzw. Zusätze zum jeweiligen Rahmenvertrag zur Regelung von Beauftragungen.

15 In der Eigentümerstrategie der aws gibt es keinen Verweis auf jährliche Arbeitsprogramme. 
Abbildung 29: $\quad$ Steuerungskanäle der Ressorts vis-à-vis den Agenturen

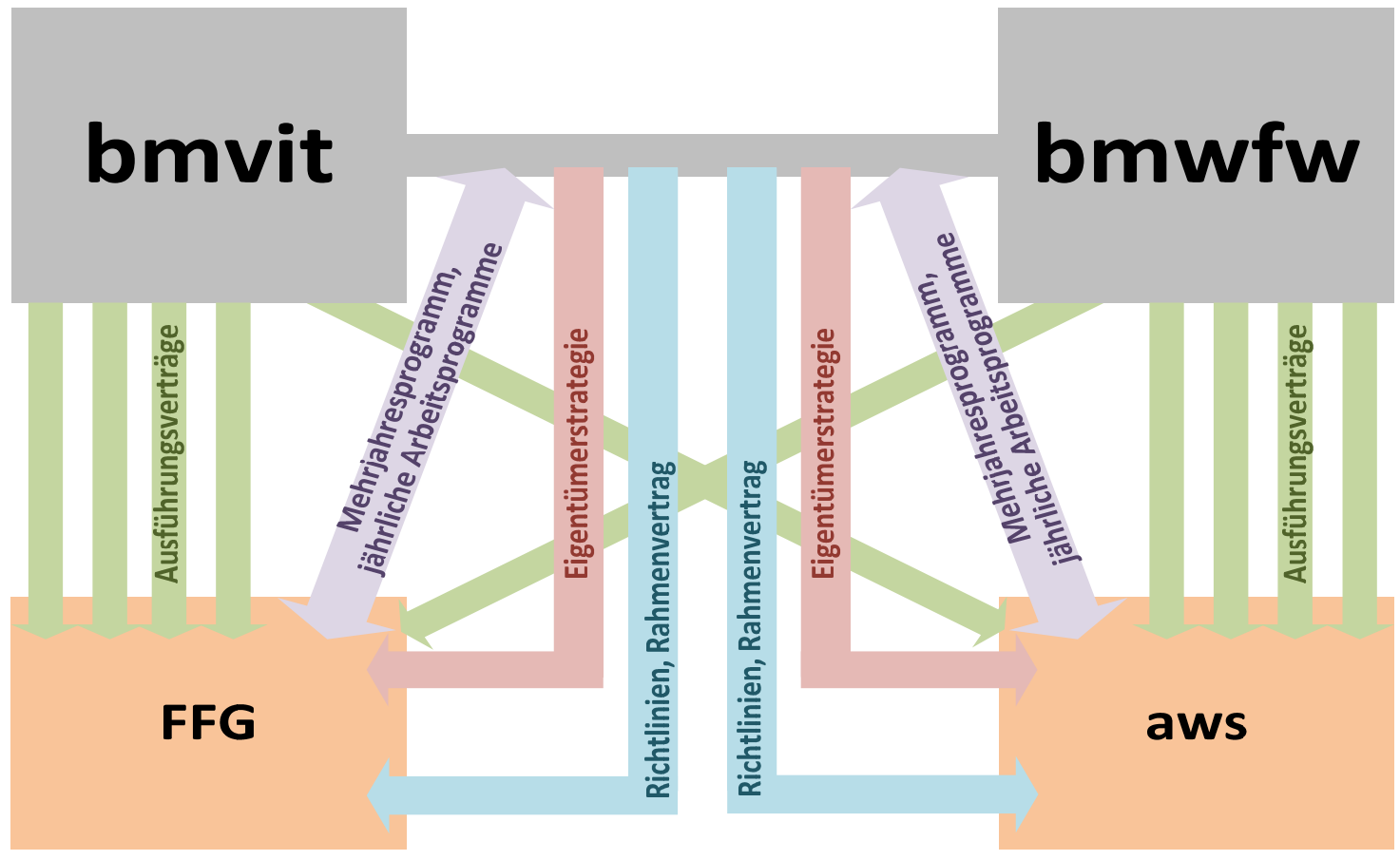

Quelle: KMU Forschung Austria 2017

Damit stehen also prinzipiell zwei Steuerungskanäle zur Verfügung: die Entwicklung und Ausdifferenzierung der Rahmenbedingungen (Eigentümerfunktion) und die direkte Beauftragung (Auftraggeberfunktion).

Ein erster Befund bezüglich der Eigentümerfunktion ist allerdings, dass die Eigentümerstrategien als Steuerungsinstrument wenig geeignet erscheinen und konsequenterweise auch weitgehend ungenutzt bleiben, da sie nicht in ausreichendem Ausmaß handlungsleitend sind. Die darin angelegten Rahmenbedingungen sind zu allgemein oder zu wenig an den tatsächlichen Handlungsprinzipien und -realitäten ausgerichtet. Auch die enthaltenen Kennzahlen (z.B. Kunden- und Mitarbeiterzufriedenheit, time to contract, Overheadkostenanteil) sind auf die Agenturen als Ganzes und daher nur in geringem Ausmaß an der Wirkung ausgerichtet. Zusätzlich werden sie von programmbezogenen Indikatoren hinsichtlich der Relevanz und ihres Nutzwertes überlagert. Die Eigentümerstrategien definieren weder die Rolle der Eigentümerressorts/Agenturen noch welches Steuerungsverständnis die Gestaltung dieser Rollen sowie die Zusammenarbeit 
leiten soll in einem Ausmaß, das sie als Instrument der Governance nutzbar erscheinen lässt16.

Außerhalb der Eigentümerstrategien verfügen die Eigentümerressorts mit der Gestaltung der Rahmenbedingungen in Form der Richtlinien und Rahmenverträge über ein relativ mächtiges Instrument der Governance bzw. Steuerung. Hier liegt eine explizite Stärke der Ressorts in der Steuerung der Agenturen. Allerdings sind die darin definierten Rahmenbedingungen relativ weich bzw. beziehen sich hinsichtlich der expliziten Governance vor allem auf (administrative) Prozesse. Der entsprechend vorhandene Interpretationsspielraum ist es auch, der diese Steuerungsmechanismen in der Einschätzung der handelnden Personen und Organisationen als relativ unkompliziert, unbestritten, kooperativ und erfolgreich erscheinen lässt.

Zentral für die Wahrnehmung der Eigentümerfunktion ist die Abnahme der von den Agenturen selbst entwickelten Mehrjahresprogramme. Diese sind zumindest konzeptionell als inhaltlich-thematischer und budgetärer Ausdruck der über die Governance gemachten Vorgaben der Ressorts zu verstehen. Allerdings ist wie bereits erwähnt, die Rolle der Ressorts weitgehend auf die Zustimmung zu diesen Mehrjahresprogrammen reduziert. Während die in ihrer Erarbeitung erfolgende Antizipation der Eigentümerposition ein indirektes Steuerungsinstrument darstellt, stehen diese Programme keineswegs als aktives Element der Governance zur Verfügung.

Nicht zuletzt aufgrund der relativ schwachen oder überwiegend indirekten, weichen Steuerung durch die Eigentümerfunktion steht die bereits erwähnte Funktion als Auftraggeber im Vordergrund jeder Diskussion über das Thema Governance. Diese überwiegt nicht zuletzt auch im Sinne der Dominanz über das Tagesgeschäft aller beteiligten Organisationen. Das wichtigste Instrument der Governance sind dabei zahlreiche Ausführungsverträge, die die Umsetzung der einzelnen Programme und der dazugehörigen Ausschreibungen (d.h. jede Ausschreibung auch innerhalb eines länger laufenden Programm erfordert einen eigenen Vertrag) regeln, also grundsätzlich alles, was nicht in Rahmenverträgen, Richtlinien oder Gesetzen bereits widerspruchsfrei definiert ist. Diese sind aufgrund ihrer hohen laufend in Gebrauch befindlichen Anzahl, aber auch hinsichtlich ihres Inhalts und der Regelungstiefe aufwändig und bürokratisch, d.h. sie binden auf beiden Seiten Ressourcen, die in Folge für die eigentlichen Aufgaben der operativen

16 So enthält die Eigentümerstrategie der aws folgende Passage „Um diese Ziele zu erreichen, sind den Gesellschaften zum einen mehr Autonomie zu geben und größtmögliche Spielräume einzuräumen. Die Exekutive nimmt sich bewusst aus der operativen Führung zurück." Ein solch klares Bekenntnis zu einem Steuerungsverständnis im strategischen Sinne fehlt in der Eigentümerstrategie für die FFG und die Befunde weisen darauf hin, dass es auch in Bezug auf die aws keine vollständige Umsetzung dieses Prinzips erfolgt. 
Umsetzung und strategischen Steuerung nicht zur Verfügung stehen. Trotz durchaus unterschiedlicher Einstellungen zu der Frage, wie eng die Agenturen geführt werden sollten, ist die Haltung der beteiligten Akteure bezüglich der Folgen des derzeitigen Systems von Einzelbeauftragungen durchweg eine eher ablehnende.

Abgesehen von der Komplexität und dem Aufwand, die mit den Ausführungsverträgen verknüpft sind, entstehen andere, relevante Nachteile für die Leistungsfähigkeit der gesamten Akteurslandschaft. Das System erlaubt einer immer mehr an Schnelligkeit (und damit auch Schnelllebigkeit) sowie Sichtbarkeit orientieren Politik den direkten, tiefen Eingriff in die Agenturen und kann dort teilweise Auslöser signifikanten Koordinationsaufwands sein (etwa die Abstimmung mit anderen Akteuren oder auch innerhalb des eigenen Portfolios) bzw. führt zu einer Reduktion von agenturinterner Planbarkeit sowie einer verstärkten Unsicherheit. Die Tatsache, dass Bedingungen der Zusammenarbeit und Governance auf Programmebene geregelt sind, ist Ergebnis des uneinheitlichen Rollenverständnisses und verstärkt die dadurch entstehenden Unklarheiten in Bezug auf die Arbeitsteilung. Damit verknüpft ist der Umstand, dass die Steuerung der Agenturen als Ganzes so gar nicht möglich ist bzw. dass Steuerung vielfach durch informelle Kanäle erfolgt und auf individuelle Lösungen angewiesen ist. Es ist in diesem Zusammenhang allerdings auch von Bedeutung, dass es vielfach Evidenz für eine effektive und kooperative Governance eben auf der Basis solcher Einzellösungen gibt.

Die Steuerung der Agenturen durch die Ressorts basiert auf keinem erkennbar einheitlichen, koordinierten oder auch nur diskutierten Steuerungsverständnis, d.h. je nach Kontext kommen unterschiedliche Mechanismen und Steuerungskanäle zum Einsatz. Es scheinen ganz unterschiedliche normative Vorstellungen darüber zu existieren, wie Governance in einem von Agencification (sei sie noch so unvollständig) geprägten System auszusehen hat. Darüber hinaus sind die Rahmenbedingungen für einzelne Organisationsteile - agentur- und ressortseitig - so unterschiedlich, dass die Diskussion normativer Werthaltungen und entsprechend ausgestalteter Verwaltungs- und Förderungspraxis u.U. auch auf gar keinen gemeinsamen Nenner kommen würde. Die Steuerung ist in einzelnen Fällen nur teilweise, in anderen Fällen gar nicht auf die Wirkung der jeweiligen Maßnahme ausgerichtet. Vorhandene Instrumente einer strategischen Steuerung (siehe Diskussion der Eigentümerfunktion) werden kaum genutzt, was (auch) an einzelnen Personen hängt, die aus historischen Gründen eine operative Involvierung bevorzugen. Zwischen der Konzeption und Wahrnehmung der Eigentümerrolle und Auftraggeberrolle herrscht eine deutliche Diskrepanz, die sich u.a. darin äußert, dass etwa die Eigentümerstrategie der aws klar davon ausgeht, dass es notwendig ist "[...] den Gesellschaften [...] mehr Autonomie zu geben und größtmögliche Spielräume einzuräu- 
men. Die Exekutive nimmt sich bewusst aus der operativen Führung zurück." und gleichzeitig ein System von Einzelbeauftragungen existiert, das diesem Credo teilweise widerspricht.

Auch bezüglich der Steuerung durch Eigentümervertretung und Auftraggeberrolle, ist festzuhalten, dass die Situation durch die steigende Bedeutung der Kabinette und dem direkten Zugang der Agenturen hierhin zunehmend komplexer wird. Damit entsteht eine Konkurrenzsituation zwischen Kabinetten und Fachabteilungen, die vor allem die Agenturen für sich zu nutzen wissen bzw. wodurch sie teilweise in eine Mediatorenrolle gedrängt werden. Die Agenturen geraten damit in eine zunehmend stärkere Position, was sie als Ganzes auch weniger leicht steuerbar macht. Allerdings zeigen die Ergebnisse der MitarbeiterInnenbefragung klar, dass dies eine uneinheitliche Entwicklung ist. Es gibt einen etwa gleich großen Anteil an RessortmitarbeiterInnen, der die Steuerbarkeit der Agenturen seit ihrer Schaffung als verbessert bzw. als verschlechtert wahrnimmt. Grundsätzlich ist dabei das System der Einzelbeauftragungen der zentrale Steuerungskanal, der zur Verfügung steht, um die Fähigkeit zur Steuerung aufrechtzuerhalten.

Insbesondere in Bezug auf die Frage der Steuerung der Agenturen durch die Ressorts wird auch deutlich, dass die Mehrheit der in diesem System aktiven Akteure keine Veränderung infolge der Systemevaluierung und der FTI-Strategie der Bundesregierung beobachten konnte. Dahinter stehen zwei Befunde über die Auswirkung der Rahmenbedingungen auf das Governancesystem: Zum einen gehen einige Befragte davon aus, dass systematische Veränderungen nur in Kombination mit steigenden Budgets realistisch sind und dass eben diese spätestens ab 2012 nicht zu verzeichnen waren. Zum anderen scheint hinter den ausgebliebenen Veränderungen auch eine gewisse Beharrung der handelnden Personen auf dem Status quo zu stehen. Für einige führt dies sogar zu dem Schluss, dass eine echte Modifikation des Verhältnisses zwischen Agenturen und Ressorts erst dann erfolgen kann, wenn diese nicht länger Schlüsselpositionen im System besetzen. 


\subsection{Einfluss und Steuerbarkeit}

Die Frage, wie Einfluss und letztlich Macht 17 im Wechselspiel zwischen Ressorts und Agenturen verteilt sind bzw. wie sie ausgeübt werden, kann über die Analyse der Governance hinaus als Bewertungsaspekt dienen. Nicht zuletzt tragen die entsprechenden Befunde zur Beantwortung der Frage bei, wer sich in den diskutierten Strukturen letztlich durchsetzen kann und inwieweit es seit der Strukturreform eine Verschiebung gegeben hat.

Wie bereits erwähnt, sehen viele Akteure das Größenwachstum und den Bedeutungszuwachs der Agenturen aufgrund von übernommenen Aufgaben etc. als zentrales Element der Governance und damit auch der Frage nach deren Einfluss sowie Steuerbarkeit. Für einige haben FFG und aws inzwischen eine Monopolstellung erreicht, was nicht zuletzt aufgrund der Arbeitsteilung zwischen den Agenturen nur konsequent erscheint. Dieses Monopol bezieht sich allerdings nicht ausschließlich auf die Tatsache, dass bestimmte Aufgaben nur von einer der beiden Agenturen ausgeführt werden können, sondern explizit auch auf die Position der Agenturen vis-à-vis den Fördernehmern und dem Wissen (strategische Intelligenz und praxisbezogenes Wissen über Abwicklung, Wirkungen etc.), das aus dem direkten Kontakt mit den Fördernehmern und ihren Projekten entsteht. Die Kombination aus der (auch finanziellen) Größe der Agenturen und dem Informations- und Wissensvorsprung lässt sie als Ganzes aus der Sicht einiger RessortmitarbeiterInnen de facto unsteuerbar erscheinen. Dazu trägt auch die Einschätzung bei, dass es keine entsprechende Entwicklung aufseiten der Ressorts gegeben hat, d.h. es gibt keine Ressourcen- oder Mitarbeiterverschiebung in Richtung steuernde Aufgaben vis-à-vis den Agenturen.

Ein zentrales Element der Verteilung von Einfluss ist die bereits diskutierte Stärke der Kabinette in den beiden Eigentümerressorts und die Zunahme des Gewichts ihrer Interventionen bzw. ihrer Funktion als Kommunikationspartner. Inzwischen verlaufen viele Kommunikationskanäle, auch zu Abstimmungen konkreter Förderinitiativen, nicht länger zwischen Agenturen und FachbeamtInnen, sondern zwischen den GeschäftsführerInnen der Agenturen und den MitarbeiterInnen der Ministerkabinette. Damit einher geht ein signifikanter Verlust an Einfluss für die FachbeamtInnen. Es gab laut den interviewten Akteuren in Ressorts und Agenturen in der jüngeren Vergangenheit mehrere Programme, die von FachbeamtInnen entwickelt bzw. konzipiert und durch den direkten

17 Durchaus in der Definition von Max Weber zu verstehen: "[...] jede Chance, innerhalb einer sozialen Beziehung den eigenen Willen auch gegen Widerstreben durchzusetzen, gleichviel, worauf diese Chance beruht" (Weber (1921): Wirtschaft und Gesellschaft. Grundriß der verstehenden Soziologie, 1. Halbband, Tübingen, S. 28) 
Kontakt der Agenturen zu den Kabinetten verhindert oder als Input des Kabinetts durch denselben Kanal an den FachbeamtInnen vorbei an die Agenturen beauftragt wurden. Dennoch scheint es zumindest theoretisch möglich, dass die FachbeamtInnen diese Konstellation auch nutzen können, um Programmideen ohne Beteiligung der politischen Ebene der Ministerien in einer Agentur zu platzieren. Letzteres ist aber wohl nur im Ausnahmefall möglich gewesen.

Während also der beobachtete Regelfall eine Machtverschiebung weg von den FachbeamtInnen zu bedeuten scheint, dürfte die Situation vor allem volatiler und insgesamt unsicherer geworden sein, insbesondere innerhalb der Ressorts, wodurch die Position der Agenturen gestärkt wird. Auch darin liegt eine mögliche Begründung für die häufig anzutreffende Einschätzung, dass vor allem die Agenturen von den Veränderungen der letzten Jahre profitieren konnten, indem ihr Einfluss deutlich gewachsen ist. Der Befund in bestimmten Bereichen und Themen anhaltend praktizierten Mikromanagements durch die Ressorts wird teilweise als Reaktion auf diese Verlagerung des Einflusses bzw. die zunehmende Unklarheit und Unsicherheit gewertet.

Diese zunehmende Volatilität/Unsicherheit bzw. die dahinterliegende ungleiche Entwicklung, die zu einem System paralleler Existenz vollkommen unterschiedlicher Einflusskanäle, Arbeitsteilungen und Steuerungsmechanismen führt, zeigt sich auch in der Interpretation der Veränderungen hinsichtlich des eigenen Handlungsspielraums der Akteure. Dabei sind zwei Erkenntnisse besonders auffallend: Zum einen gibt es Personen in den Ressorts, die trotz der zugenommenen Autonomie der Agenturen sowie des eigentlichen Strukturprinzips der Verlagerung der Umsetzung von Maßnahmen in die Agenturen einen Zuwachs an Handlungsspielraum in der Umsetzung seit der Schaffung von FFG und aws erleben. Zum anderen spiegelt auch diese Analyse die heterogene Entwicklung wider, insofern als sowohl hinsichtlich Programmdesign als auch -abwicklung nahezu gleichgroße Anteile der MitarbeiterInnen von Agenturen und Ressorts eine Vergrößerung bzw. eine Verringerung ihrer Handlungsmöglichkeiten beobachten.

Ein wesentlicher Faktor für gestiegenen Einfluss der Agenturen besteht nicht zuletzt in der geringen Koordination zwischen den Eigentümerressorts. Dabei ist insbesondere auf die Uneinigkeit darüber zu verweisen, wie die Agenturen im Regelfall (d.h. durchaus auch je nach betroffenem Politikfeld) zu steuern sind bzw. von wem entsprechende Steuerungsimpulse auszugehen haben. Das Prinzip der geteilten Eigentümerschaft hat seit der Schaffung der FFG und aws nicht zu einer ausreichenden Abstimmung der Ressorts untereinander geführt, was ihren Einfluss gegenüber den Agenturen zusätzlich 
schwächt. Dieser Sachverhalt ist jedoch kein österreichisches Spezifikum. Auch auf internationaler Ebene stellt die Steuerungsfähigkeit der Ministerien generell eine größere Herausforderung dar als die Steuerbarkeit von Agenturen.18

Insofern als die Zuordnung finanzieller Mittel eines der stärksten Steuerungsinstrumente darstellt, muss in diesem Zusammenhang auch die Rolle des BMF betrachtet werden. Faktisch hat das Finanzministerium aufgrund der Hoheit auch über bereits beschlossene Budgets einen der wesentlichen Einflussmechanismen in der Hand und nutzt den entsprechenden Kontroll- und Entscheidungsspielraum im Sinne der eigenen Interventionslogik. Aus der Sicht vieler Akteure hat es im BMF einen Wandel weg von einer wirtschaftspolitisch gestaltenden Organisation hin zu einer fast ausnahmslos an der Haushaltsdisziplin orientierten Institution vollzogen. Damit wird es als mächtiger Partner in, mit Ausgaben und Unsicherheiten verbundenen, Politikfeldern zu einem Einzelakteur, der Entscheidungen teils nachträglich entwertet bzw. auch die Funktionsfähigkeit der Agenturen und des Systems - die sich ja vis-à-vis den Fördernehmern in der Schnelligkeit und Tatsächlichkeit der Mittelauszahlungen ausdrückt - entscheidend beeinflusst. Ob aktiv oder über die Antizipation der handelnden Personen vor allem in den Ressorts, ist das BMF ein entscheidendes Element in dem gesamten Einflussgefüge.

\subsection{Bewertung vor dem Hintergrund des Prinzipal-Agent Problems}

Als analytischer Hintergrund für die oben diskutierten Befunde diente die PrinzipalAgent-Theorie. Daher sind die gewonnenen Erkenntnisse auch dahingehend zu analysieren, inwieweit die potenziellen Probleme einer solchen durch Agencificationsprozesse entstandenen Situation den theoretischen Herausforderungen entsprechen.

\section{Moral hazard}

Moral hazard beschreibt die Unsicherheit des Prinzipals darüber, ob der Agent auch wirklich das Richtige und Notwendige tut, um seine (des Prinzipals) Ziele zu verwirklichen. Der Agent hat demzufolge Interesse daran, seinen Aufwand zur Erbringung der

18 Tobias Bach, Department of Political Science, University of Oslo bei den Technologiegesprächen in Alpbach (Session 02 „The promotion of research and innovation in Austria: governance between management and autonomy“) , 25.08.2017; siehe auch: Schillemans, T./ Busuioc, M. (2015). Predicting Public Sector Accountability: From Agency Drift to Forum Drift. Journal of Public Administration Research and Theory 25 (1):191-215 sowie Bach, T./Fleischer, J./Hustedt, T. (2010). Organisation und Steuerung zentralstaatlicher Behörden: Agenturen im westeuropäischen Vergleich. Berlin: Edition Sigma. 
vereinbarten Leistungen so weit zu reduzieren, dass der Prinzipal gerade noch zufrieden gestellt werden kann. Damit der Agent diese Vorgehensweise anwenden kann, ist es für inn sinnvoll, den Prinzipal nicht vollständig über Handlungsmöglichkeiten und Lösungsoptionen zu informieren.

Unsicherheiten über die Leistungsfähigkeit und -willigkeit der Agenturen seitens der Ressorts scheinen ganz überwiegend nicht zu existieren, wobei es Einzelbefunde gibt, die genau das, also die willentliche und wissentliche Nicht-Performanz der Agenturen unterstellen. Allerdings wird die eigentliche Leistung, d.h. jene gegenüber den Fördernehmern von keinem Akteur ernsthaft in Frage gestellt, weder in ihrer Effizienz noch Effektivität, Transparenz etc. Dennoch gibt es vereinzelt Unsicherheit darüber, ob die Agenturen in Teilen nicht einer eigenen Interpretation der beauftragten Leistung gegenüber den Ressorts den Vorrang geben und somit bei letzteren den Eindruck vermitteln, dass die vollständige Delegation aller Umsetzungsaufgaben schon daher nicht erfolgen kann, weil sonst bestimmte Aufgaben nicht (mehr) ausgeführt würden. Dies verweist auf teilweise mangelndes Vertrauen in die Gewichtung der zu erbringenden Leistungen der Agenturen.

In der Theorie folgt aus einer solchen Situation, dass zur Reduktion der Unsicherheit Verträge geschlossen werden, die die zu erbringenden Leistungen des Agenten fixieren und festlegen, welche Entlohnung, Kontrollmaßnahmen etc. vorgesehen sind. Obgleich echte Unsicherheiten, die auf moral hazard verweisen, kaum bzw. wenn dann nur in Einzelfällen vorzuliegen scheinen, dürfte das umfangreiche System von Verträgen (von Rahmen- hin zu Ausführungsverträgen für einzelne Ausschreibungen) generell Ausdruck solcher Unsicherheiten sein. Es lässt sich dementsprechend argumentieren, dass die bestehenden Governancestrukturen und -regeln Ausdruck einer inhärenten und weit verbreiteten Unsicherheit der Ressorts über die Leistungen der Agenturen sind, die nur wenig Entsprechung in der Realität finden. Inwiefern die Realität zahlreicher Verträge auch als selbstverstärkende Ursache für diese Unsicherheit funktioniert, lässt sich nicht abschließend bewerten. Allerdings erscheint es angesichts der realen Zusammenarbeit von FFG, aws, bmvit und bmwfw nicht nur aus Ressourcengründen, sondern vor allem auch aus Governanceperspektive sinnvoll, die Vielzahl dieser, ohnehin nicht vollständig als Kontrollinstrument geeigneten, Verträge deutlich zu reduzieren. Im Umkehrschluss müssten bestehende (oder neue) Verträge dann aber eine echte Kontrolle ermöglichen und über die eingebaute Möglichkeit von Sanktionen die Basis für mehr Vertrauen und mehr Sicherheit bieten. 


\section{Hidden information und Informationsasymmetrien}

Asymmetrische Informationen liegen im Wesenskern jeder Prinzipal-Agent-Konstellation und treffen auch auf die hier analysierte Beziehung zwischen den Ministerien und Agenturen zu. Im theoretisch-konzeptionellen Verständnis bezieht sich dies darauf, dass der Prinzipal die Handlungen des Agenten zur Leistungserbringung zwar beobachten, deren Qualität aber (z.B. aufgrund mangelnder Fachkenntnis) nicht einschätzen kann. Im vorliegenden Fall bezieht sich diese Problematik bzw. deren Basis auf die ungleiche Verteilung von strategischer Intelligenz (bzw. den Ressourcen, die zu deren Aufbau notwendig sind) und teilweise auch von Informationen zur Wirksamkeit von Maßnahmen über Evaluierungen und Monitoringsysteme (und damit indirekt zur Leistung der Agenturen). Obgleich letzteres zu den Kernaufgaben der Ressorts gehören sollte, gibt es Fälle, in denen eine Programmevaluierung entweder ohne Ressortbeteiligung beauftragt und durchgeführt wird oder eine externe (und damit Unabhängigkeit signalisierende) Bewertung durch eine agentur-interne Lösung ersetzt wird. Für den Aufbau strategischer Intelligenz gilt dies in abgeschwächter Form.

Für beide Befunde gilt, dass durch die zunehmende Rolle der Ministerkabinette als Ausdruck und Treiber eines beschleunigten Politikzyklus sowie der steigenden Bedeutung von Sichtbarkeit gleichsam als Nachweis politischer Aktivität ein dritter Akteur in die bestehende Prinzipal-Agenten-Konstellation eintritt. Damit entsteht eine Situation, in der nicht länger eindeutig klar ist, wer letztlich die Rolle des Prinzipals einnimmt und dementsprechend die Vorgaben für die Agenten/Agenturen macht. Anstatt also Unsicherheiten für alle Beteiligten zu minimieren, entstehen eher zusätzliche. Das bereits in der Systemevaluierung attestierte Steuerungsvakuum, d.h. die Abwesenheit koordinierter, verbindlicher Steuerung der Agenturen durch die Ministerien und die teilweise konträren Signale über Eigentümerfunktion einerseits und Auftraggeberfunktion andererseits, verschärft sich und erlaubt den Agenturen zusätzlichen Handlungsspielraum, den sie für sich zu nutzen wissen, indem sie Steuerungsimpulse (d.h. konkrete Idee für Fördermaßnahmen) über verschiedene Kanäle abwenden. Dies ist in erster Linie jedoch eine konkrete Reaktion auf das uneinheitliche Vorgehen der jeweilig auftretenden Prinzipale.

In Summe kann davon ausgegangen werden, dass die untersuchte Governance zwischen den beiden Förderungsagenturen und ihren Eigentümern/Auftraggebern durchaus Elemente unerwünschter und dennoch mit der Schaffung einer Prinzipal-AgentenKonstellation nahezu automatisch verbundener Eigenschaften aufweist. Diese werden in der Praxis von einem in vielen Fällen gut funktionierenden System individuell bzw. bilateral ausgehandelter Interpretationen überlagert. Insgesamt scheint so gewährleistet, dass die diskutierten Probleme von eher nachrangiger Bedeutung für die Leistungsfähigkeit des Systems bleiben. Allerdings werfen diese einzelnen Lösungsansätze schon 
aufgrund der Notwendigkeit ihrer Existenz mögliche Fragen über die Leistungsfähigkeit des Systems an sich auf, d.h. es ist eine auch bereits in der Systemevaluierung festgehaltene Auseinanderentwicklung der strukturellen Verfasstheit der Prinzipal-AgentKonstellation einerseits und der stark individualisierten und auf individuelles Handeln beruhenden Praxis andererseits zu konstatieren.

\subsection{Fazit}

Ein zentraler Befund der vorliegenden Evaluierung hinsichtlich der Steuerung lautet, dass das gesamte Governancesystem von (anhaltend) hoher Komplexität geprägt ist. Dies ist einerseits dem Umstand geschuldet, dass die Steuerung der Agenturen nach wie vor über zahlreiche Ausführungsverträge erfolgt, die für alle Beteiligten sehr aufwendig und ressourcenbindend ist. Andererseits existiert kein einheitliches koordiniertes Steuerungsverständnis seitens der Eigentümer, und den Ressorts fehlt es an einer klaren, handlungsleitenden Eigentümerstrategie. Nicht zuletzt daher lassen sich divergierende Selbst- und Fremdbilder bei der Aufgabenteilung zwischen Ressorts und Agenturen beobachten. Allerdings entsprechen diese divergierenden Rollenzuschreibungen letztlich der Tatsache einer unvollständigen Delegation von Aufgaben an die Agenturen. Die Steuerung der Agenturen geht von unterschiedlichen Ministerien (zumindest den beiden Eigentümerressorts) mit ihren jeweiligen Fachabteilungen und Kabinetten aus. Die verschiedenen Steuerungsimpulse nutzen unterschiedliche Steuerungsmechanismen und -kanäle (etwa Weisungen an die jeweilige Geschäftsführung, Beauftragung von Programmen, Prüf- und Kontrollrechte etc.) und sind in der Regel nicht abgestimmt und zum Teil sogar widersprüchlich. Insgesamt entsteht auch vor dem Hintergrund der relativ großen und weiterwachsenden Bedeutung der Agenturen (in personeller, finanzieller Hinsicht) und nicht zuletzt ihrer jeweiligen Monopolstellung der Eindruck, dass eine Steuerung der Agenturen als Ganzes aufgrund der relativen Steuerungsschwäche der Ressorts derzeit kaum möglich ist. Vielmehr scheint ein Steuerungsvakuum zu existieren, das am ehesten noch die Agenturen für sich zu nutzen wissen. Der vielfach geäußerte Vorwurf häufigen Mikromanagements durch die Ressorts erklärt sich nicht zuletzt als Reaktion auf die wahrgenommene (eigene) Steuerungsschwäche.

Während die Agenturen auch in weitgehend unveränderten Rahmenbedingungen aufgrund etwa der Kumulation strategischer Intelligenz und des Zuwachses an Einfluss stärker bzw. autonomer geworden sind, hat es weder eine adäquate Neujustierung des Systems (auch nicht infolge der Systemevaluierung) gegeben noch eine umfassende analoge Ko-Evolution innerhalb der Ressorts. Letzteres betrifft vor allem die ausgebliebene Entwicklung eines neuen Steuerungsverständnisses und entsprechender Einstellungen 
sowie Fähigkeiten vor dem Hintergrund zunehmend professionalisierter Umsetzungsprozesse in den Agenturen.

Für die Bewertung der Steuerung und Governance sind darüber hinaus vor allem die in den Strukturreformen formulierten Zielsetzungen relevant. Dabei ist zu beachten, dass das Ziel einer optimierten Governance und effektiveren Steuerung in den Strukturreformzielen nur indirekt adressiert wurde. Dennoch können die gewonnenen Erkenntnisse einen Beitrag zur Frage nach der Erfüllung der vier Ziele leisten.

Eine Komplexitätsreduktion in organisatorischer Hinsicht erscheint auf den ersten Blick schon dadurch erreicht, dass es anstatt acht Organisationen nur mehr zwei Agenturen gibt. Vor dem Hintergrund anhaltend unterschiedlicher Rahmenbedingungen und trotz der Bemühungen durch diverse Prozesse, die Vorgängerorganisationen von FFG und aws zu jeweils einer Organisation werden zu lassen, spricht die Realität der Governance als Ausdruck der entsprechenden Rahmenbedingungen jedoch eine andere Sprache. Zum einen verhindern stark unterschiedliche Finanzierungssettings die Vereinheitlichung von Steuerung, von der finanziellen (Teil-)Autonomie der FFG-Basisprogramme über die an einzelne Ausschreibungen geknüpften Finanzierungsströme in anderen Bereichen bis hin zur Komplexität der aws Garantieprogramme mit ihrer Verflechtung von Auftraggebern, Eigentümern, Budgetkontrolle durch das BMF und dem Verbleib des Ausfallsrisikos ebenfalls im Finanzministerium.

Zum anderen sind das Rollenverständnis und damit einhergehend die Arbeitsteilung als Basis der Governance auch durch die unterschiedlichen Formen der geistigen Eigentümerschaft sowie des intrinsischen Interesses einzelner Personen an Themen etc. geprägt. Vor allem missionsorientierte Ansätze bedürfen aus Sicht der Ressorts scheinbar einer stärkeren Involvierung in die Umsetzung durch die Agenturen. Letztlich führt dies vor allem zu einer Ausdifferenzierung der nutzbaren und genutzten Steuerungskanäle, was zumindest aus der Steuerungsperspektive der Einschätzung widerspricht, dass die Agenturen inzwischen als jeweils eine Organisation operieren (können). Das Auseinanderfallen in der Wahrnehmung der Eigentümer- und Auftraggeberrolle seitens der Ministerien, die Zunahme direkter Steuerung durch die Kabinette (und damit abseits der tradierten Zusammenarbeit zwischen Agenturbereichen und Fachabteilungen in den Ressorts) sowie die Rolle des BMF tragen ebenfalls nicht zur Reduktion organisatorischer Komplexität bei.

Die Reduktion inhaltlicher Komplexität erscheint nicht nur im Hinblick auf die Governance ein unerreichbares Ziel zu sein. Die Ausdifferenzierung der Politik- und Interventionsfelder, die steigende Internationalisierung, die zunehmenden Effekte neuer Themen und 
der ständig voranschreitenden Technologieentwicklung auf die sozioökonomische Entwicklung von Volkswirtschaften etc. müssen konsequenterweise zu einer steigenden inhaltlichen Komplexität politischen Handelns führen.

Die Lösung immanenter Koordinationsprobleme als übergeordnetes Ziel der Schaffung von FFG und aws erscheint nur insofern erreicht, als die Schaffung zweier Agenturen mit soweit möglich harmonisierten Prozessen etc. die Koordination von Steuerungsimpulsen im Sinne ihrer notwendigen Ausgestaltung sicherlich erleichtert hat. Darüber liegt allerdings die dadurch nicht direkt thematisierte Koordination ganzer Politikfelder, deren Umsetzung (nicht vollständig) in den Aufgabenbereich der Agenturen delegiert wurde. Auf der Basis der vorliegenden Evaluierung kann dies als nur teilweise geglückt bezeichnet werden. Wie auch schon in der Systemevaluierung herausgearbeitet, ist die Koordination zwischen den Ressorts zwar strukturell geregelt, in der Realität aber unterentwickelt und nach wie vor teilweise in die Agenturen verlagert, d.h. letztere übernehmen die Funktion der Abstimmung und der Diskussion der von den Ressorts unabhängig voneinander an sie herangetragenen Wünsche und konkreten Programmideen, Beauftragungen etc.

Die avisierte Verbesserung der Umsetzungschancen von Regierungszielen kann als überwiegend erreicht betrachtet werden. Dies ist allerdings weniger eine Funktion des Governancesystems, sondern vor allem der Professionalisierung der Agenturen hinsichtlich der eingesetzten Prozesse. Darüber hinaus ist es gelungen, eine, wenngleich sehr unterschiedliche, Arbeitsteilung zwischen Ministerien und Agenturen zu etablieren und Modi der Zusammenarbeit zu entwickeln, die innerhalb der jeweiligen Themen etc. relativ stabil sind. Allerdings basiert dieses System nach wie vor zu stark auf Individuen und ihren bilateralen Übereinkünften, d.h. eine systematische und systemische Umsetzung entsprechender Governancemechanismen und Steuerungskanäle hat nicht stattgefunden und wird aktuell von Entwicklungen (z.B. die stärkere Involvierung der Kabinette und teilweise Aushebelung tradierter Kommunikations- und Steuerungskanäle) dominiert, die zu mehr Unsicherheit und Volatilität führen. Dies könnte zukünftig die Umsetzung von Regierungszielen möglicherweise mehr behindern als fördern. 


\section{Internationaler Vergleich: Ausgewählte Förderge- sellschaften anderer Länder}

Ziel der Betrachtung ausgewählter Fördergesellschaften in anderen Ländern war die Herausarbeitung von "good practices" in der Governance von Förderagenturen, also die rechtliche und gelebte Ausgestaltung des Delegationsverhältnisses zwischen den Regierungen und den Agenturen. Dabei ist ein direkter Vergleich bzw. ein "benchmarking" aufgrund der Diversität der nationalen Kontexte und des abgeleiteten Förderhandelns nur sehr begrenzt möglich, wie Glennie und Bound (2016) im Zuge ihres Vergleichs von zehn Innovationsagenturen herausgearbeitet haben. Sogar auf Ebene der eingesetzten Förderinstrumente ist dies kein einfaches Unterfangen, wie z.B. Technopolis (2014) im Rahmen ihrer Studie für das TAFTIE Netzwerk erfahren konnten, und sich somit auf vier häufige Instrumente beschränkten. Gleichwohl liefern die Betrachtungen wichtige Hinweise auf Herausforderungen und daraus abgeleiteten Prozesse und Lösungswege in anderen Ländern. Dem Vergleich lagen folgende Leitfragen zugrunde:

- Welche grundlegenden Governance-Strukturen kennzeichnen die Delegation?

- Wie ist die Autonomie der Agentur im Einzelnen ausgestaltet?

- Gibt es Hinweise auf Mechanismen, die eine gute Qualität der Zusammenarbeit zwischen Ministerien und Agenturen sichern, von denen auch FFG und aws lernen können?

Für den internationalen Vergleich kam eine Kombination aus Dokumentenanalysen und Telefoninterviews pro betrachteter Organisation zum Einsatz. Zur Darstellung der Förderorganisationen wurde ein Template erstellt, das auch als Strukturierung für die Leitfaden gestützten Interviews genutzt wurde. Wir haben vier ausländische Förderagenturen näher betrachtet, jeweils zwei mit Strukturähnlichkeit zur FFG und zwei zur aws. Diese Organisationen zeichnen sich durch unterschiedliche Autonomiegrade aus und genießen für ihr Förderhandeln internationales Ansehen. Im Falle von VLAIO, Belgien sowie VINNOVA, Schweden sind die Agenturen, ähnlich wie aws und FFG, aus einer Fusion von Vorgängerorganisationen hervorgegangen.

\section{Lessons learned}

VINNOVA und VLAIO (als Innovationsagenturen mit einem ähnlichen Portfolio zur FFG) repräsentieren nicht zuletzt hinsichtlich des rechtlichen Status deutlich unterschiedliche Lösungsansätze und Governancemechanismen. VLAIO ist rechtlich nicht eigenständig, sondern eine direkt, d.h. hierarchisch, dem Wirtschaftsministerium unterstellte Organisation. Gleichzeitig verbindet VLAIO den Gedanken einer direkt an das Ministerium angebundenen Agentur mit weitergehender Autonomie, die sich etwa in der weitgehend 
aufrechten Unabhängigkeit (von der Politik) einer der beiden Vorgängerorganisationen, dem iwt, äußert. Aufgrund der direkten Unterstellung erfolgt die Übertragung von Mitteln und Aufgaben nicht über Verträge etc.

Demgegenüber stellt VINNOVA den klassischen Entwurf einer weitgehend autonomen Agentur dar, mit längerfristiger inhaltlicher Budgetplanung sowie einer rein strategischen und inzwischen auf eine fast schon minimalistische Übereinkunft reduzierten Steuerung durch die Ministerien.

Dennoch sind die Autonomiegrade in den weiteren Bereichen geradezu überraschend ähnlich ausgeprägt (operational, personell, finanziell). Nicht nur VINNOVA mit ihrer stark ausgeprägten formellen Autonomie, sondern auch VLAIO kann, trotz enger Anbindung an das Ministerium, ihr Portfolio im Rahmen eines jährlichen Geschäftsplans und eines jährlichen Budgets, zwar in Abstimmung mit dem Ministerium, jedoch teilweise selbständig gestalten. Ein Unterschied besteht jedoch in der Kapazität zur Strategieentwicklung. Während VINNOVA aufgrund des langjährig aufgebauten Know-hows sowie des Vertrauensverhältnisses vonseiten des Prinzipals auch substanzielle eigene strategische Aufgaben wahrnimmt, wird VLAIO strategisch stärker vom Ministerium geführt.

Die deutsche Kreditanstalt für Wiederaufbau (KfW) und die finnische Finnvera weisen als Förderbanken inhaltliche Ähnlichkeiten zur aws auf. Beide sind allerdings hinsichtlich ihrer finanziellen Autonomie anders als die aws aufgestellt. In beiden Fällen haben die Agenturen eine Eigenmittelausstattung und Bankenstatus und können daher am Kapitalmarkt Refinanzierungsgeschäfte tätigen. Zudem verfügen beide Agenturen, anders als die aws, auch über Exportförderungen.

Dennoch können für die Governance der aws Impulse abgeleitet werden. Sowohl bei der $\mathrm{KfW}$ als auch der Finnvera gibt es trotz der weitgehenden finanziellen sowie organisationalen, operationalen und personellen Autonomie Rückkopplungsmechanismen mit den Eigentümer-Ressorts. Im Fall der KfW geschieht dies über die Vorstandswahl, die jährliche Bewertung des Vorstands, die Zustimmung zur Jahresplanung und die formelle Beauftragung, die für jedes $\mathrm{KfW}$-Instrument durch die Ministerien erfolgen muss. Bei der Finnvera findet die Steuerung fast ausschließlich über die gemeinsam ausgehandelten Mehrjahresprogramme statt. Für Transparenz sorgen hier die halbjährlichen Berichte der Geschäftszahlen sowie die (externe) Evaluation der Fördertätigkeit. Vor diesem Hintergrund haben beide Agenturen großen Einfluss auf die Gestaltung ihres Förderportfolios.

Wesentliches Element der Governance von KfW und Finnvera sind unabhängige und starke Aufsichtsgremien, deren Mitglieder von vielen unterschiedlichen Stakeholdern (Parteien bzw. Parlamente, Sozialpartner, Kunden und Zielgruppen) benannt werden. Die Eigentümer-Ressorts sind zwar in einer führenden Rolle, verfügen aber nicht über 
die Mehrheit der Stimmen. Förder- und Finanzierungsentscheidungen werden vollkommen unabhängig getroffen. Beide Agenturen haben sich aufgrund nachgewiesener Erfolge eine Vertrauensbasis mit ihren Prinzipalen geschaffen, die innen faktisch große Freiheitsgrade ermöglicht.

\subsection{Vergleich mit aws}

\subsubsection{KfW - Deutschland}

Die Kreditanstalt für Wiederaufbau (KfW) wurde 1948 gegründet und ist die größte nationale Förderbank weltweit. Sie ist als Bankengruppe organisiert und betreibt sowohl inländisches Fördergeschäft als auch internationale Programme. Ihre Aufgaben reichen von der Förderung des Mittelstands, freier Berufe, Existenzgründern, der Vergabe von Risikokapital, der Innovationsförderung, der Exportfinanzierung über die Förderung der Wohnungswirtschaft und des Umweltschutzes hin zu entwicklungspolitischen Aufgaben (vgl. §2 Abs 1. KfW-Gesetz). Vor allem die Programme der KfW-Mittelstandsbank sind mit jenen der aws vergleichbar. Der Jahresumsatz der Bankengruppe betrug 201681 Mrd. Euro, der der KfW-Mittelstandsbank 21,4 Mrd. Euro. Genau wie die aws verwaltet auch die KfW das aus dem Marshallplan stammende ERP-Sondervermögen (seit 2007). Die KfW-Bankengruppe hat knapp 6.000 MitarbeiterInnen.

Die KfW vergibt Kredite in der Regel im Durchleitungsverfahren über Hausbanken an Unternehmen und Privatleute. Garantien und Zuschüsse vergibt sie nicht.

\section{Wesentliche Aspekte der Governance}

Die KfW hat einen sehr unabhängigen Status, der sich aus dem KfW-Gesetz ergibt: Darin ist die Eigenkapitalausstattung festgeschrieben sowie das Prinzip, das keine Gewinne ausgeschüttet, sondern zur Rücklagenbildung und damit zur künftigen Finanzierung des Fördergeschäfts herangezogen werden.

Das Hauptinstrument der KfW sind Kredite. Diese kommen vom Kapitalmarkt und die KfW kann sie in der Regel zu besonders günstigen Konditionen erhalten, da das Rating des Bundes auch für sie gilt. Außerdem versucht sie die Konditionen ihrer Kredite weiter zu verbessern, d.h. günstigere Zinsen anzubieten. 
Die Finanzierung dieser Zinsverbilligung kann aus drei verschiedenen Quellen erfolgen:

- KfW-Eigenmittel, dann handelt es sich um sog. "KfW-Programme",

- ERP-Vermögen des Bundes, dann spricht man von "ERP-Programmen",

- sowie aus dem Bundeshaushalt, die sog. "Bundesprogramme".

Die Basis der Beauftragung der KfW bildet das KfW-Gesetz, das in §2 Abs. 1 die gesetzlichen Aufgaben festlegt (s.o.). Diese lassen sehr viel Spielraum, innerhalb dessen die $\mathrm{KfW}$ ihre Förderstrategie festlegt. Es gibt eine eigene Abteilung für strategische Planung, die globale Megatrends definiert (aktuell sind dies Globalisierung, Sozialer Wandel, Digitalisierung und Innovation, Klimawandel, Umwelt). Darauf baut eine mehrjährige Produktentwicklung auf und daraus wiederum leitet sich die jährliche Produktentwicklung ab.

Die Corporate Governance ist sehr autonom.

Es gibt einen Verwaltungsrat, der sehr heterogen besetzt ist. Die 37 Mitglieder sind VertreterInnen der Bundesregierung (7), des Bundestages und des Bundesrates (je 7) sowie VertreterInnen der wesentlichen Geschäftspartner, der Hausbanken, relevanter Branchen sowie der Industrie und der Gewerkschaften. Den Vorsitz teilen sich im jährlichen Wechsel die beiden aufsichtführenden Ministerien, das Bundesministerium für Finanzen (BMF) und das Bundesministerium für Wirtschaft (BMWi). Die Heterogenität des Gremiums stellt die Unabhängigkeit der Entscheidungen sicher. Dem Verwaltungsrat kommen gleichermaßen Funktionen einer Hauptversammlung sowie eines Aufsichtsrates zu. Er trifft sich drei- bis viermal jährlich.

Der Verwaltungsrat bestimmt den 6-köpfigen Vorstand für 3-6 Jahre. Die Amtsperioden der Vorstandsmitglieder laufen unterschiedlich, so dass immer eine teilweise Neuerung stattfindet. Es gibt im Vorstand einen (informellen) Parteienproporz, alle Vorstandsmitglieder haben ein Parteibuch. Der Vorstandsvorsitzende ist Primus inter pares. Im Falle einer Pattsituation im Vorstand wäre seine Stimme entscheidend, aber in der Praxis pflegt der Vorstand eine konsensuale Beschlussfassung.

\section{Ergebnisse im Überblick: Autonomiegrade}

\section{Operationale Autonomie}

- Strategische Intelligenz entwickelt die KfW selbst, indem sie Studien vergibt, aber auch aus den Ministerien kommen Impulse. Aktuelles Beispiel ist die Beteiligungsfinanzierung. Hierzu gab es eine Studie des BMWi, die einen Bedarf festgestellt hat. 
- Das (strategische) Agenda-Setting erfolgt vollständig durch die KfW. Die mehrjährige Planung wird im Haus entwickelt und vorm Vorstand beschlossen. Der Verwaltungsrat erhält sie zur Kenntnis. Die Jahresplanung wird dem Verwaltungsrat zur Genehmigung vorgelegt.

- Die Entwicklung neuer Förderinitiativen findet ebenfalls bei der KfW statt, dabei steht sie jedoch im Austausch mit den Ministerien und kann Impulse aufnehmen. Impulse werden dann umgesetzt, wenn sie im Einklang mit der selbstgesteckten Strategie stehen.

- Programmspezifische Erfolgsindikatoren werden intern entwickelt. Alle Produkte werden intern klassifiziert, wie viel sie auf die Megatrends einzahlen, die die Basis der mehrjährigen Produktplanung bilden.

- Agenturspezifische Leistungsindikatoren leiten sich aus der Strategie (Megatrends) ab, zudem muss die Bank nach dem Bankenrecht bilanzieren.

- Dokumentation, Berichterstattung: Unterschiedlich, je nach Programmtyp. Es gibt einen jährlichen Förderbericht an den Verwaltungsrat. Der ERP-Bericht geht in engem Turnus direkt ans BMWi.

- Umsetzung, Werbung und Evaluierung erfolgen komplett durch die KfW.

Abbildung 30: Operationale Autonomie der KfW

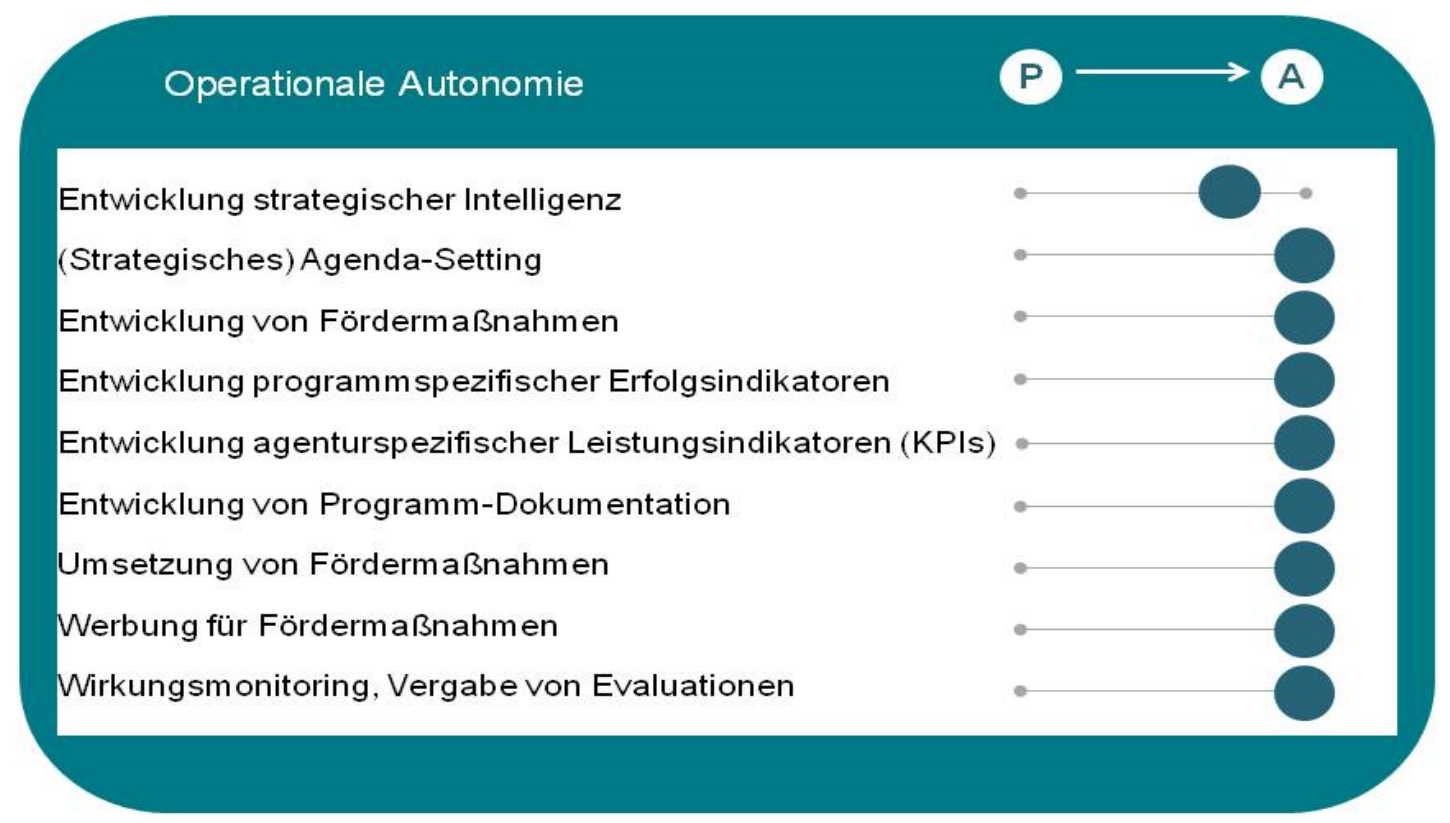

\section{Finanzielle Autonomie}

Wie oben ausgeführt, stellt der Bund nur in sehr kleinem Umfang Geld aus dem Bundeshaushalt zur Verfügung, der Großteil des Kapitals, mit dem die KfW wirtschaftet, sind Eigenmittel. Die KfW unterliegt bei ihrer Jahresberichterstattung denselben Vorschriften 
wie alle Kreditanstalten. Die Gewinne, die sie erwirtschaftet, werden nicht ausgeschüttet, sondern als Rücklagen dem Konzernvermögen zugeführt.

Die KfW kann mit ihrem Budget weitgehend autonom agieren, braucht aber dennoch für jedes Produkt KfW ein staatliches Auftragsschreiben (so legt das BMWi §2 Abs. 1 des KfW-Gesetzes aus).

Abbildung 31: $\quad$ Finanzielle Autonomie der KfW

\section{Finanzielle Autonomie}

Ergebnisorientierte Buchführung (vs. Kameralistik)

Gegenseitige Deckungsfähigkeit der Ausgabentitel

Globalbudgets (vs. Einzelbeauftragungen)

(Verbindliche) Mehrjährige Budgetplanung

Eigenmittelausstattung (vs. Zuweisungen)
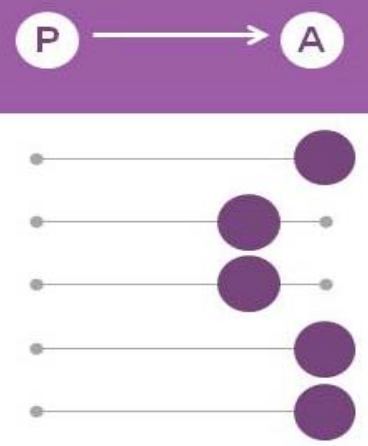

\section{Personelle Autonomie}

Der Verwaltungsrat kann aufgrund der heterogenen Besetzung nicht stark politisch beeinflusst werden (siehe auch die Ausführungen zu Verwaltungsrat und Vorstand oben). Der Verwaltungsrat bestimmt den Vorstand, in der Regel auf Vorschlag der Verwaltungsratsvorsitzenden (BMWi oder BMF) und bewertet auch die Arbeit der Vorstände. Als eigenständige Organisation betreibt die KfW ihre Personalpolitik vollkommen autonom.

Abbildung 32: $\quad$ Personelle Autonomie der KfW

Personelle Autonomie

Besetzung des Aufsichtsrates

- Tatsächliche Machtverhältnisse im Aufsichtsrat

Auswahl der GeschäftsführerInnen ( $m$. Weisungsrecht)

Personalpolitik
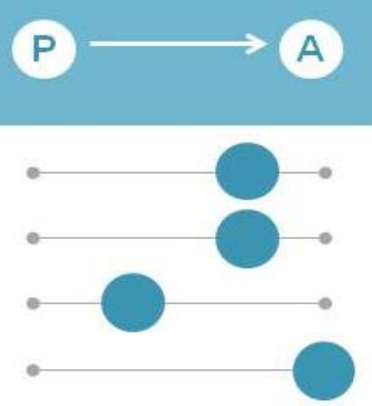


\section{Organisationale Autonomie}

Die organisationale Autonomie ist durch die eigene Rechtspersönlichkeit gegeben. Auch die Gestaltung der Aufbau- und Ablauforganisation liegen in den Händen der KfW.

\section{Qualität der Zusammenarbeit}

Die Anreizmechanismen der KfW, den politischen Auftrag auszuführen sind beispielsweise in der Vorstandsauswahl und in der Vorstandsbewertung sichtbar. Die mit der Rechtsaufsicht betrauten Ministerien BMF und BMWi diskutieren die Förderleistungen des Jahres und bewerten den Vorstand, insbesondere den Vorsitzenden. Die Vergütung und auch Vertragsverlängerungen hängen davon ab und werden am Ende vom Ministerium abgezeichnet. Häufig kommen die Vorstände aus der Politik bzw. werden von der Regierung vorgeschlagen.

Prozesse gegenseitigen Lernens sind kein herausragendes Charakteristikum der Beziehung von Regierung und KfW, finden aber durchaus informell im Rahmen der Berichterstattung sowie im Rahmen der Produktentwicklung statt.

Weiterer förderlicher Faktor für die Autonomie bzw. Mechanismen, die die Dominanz von Gruppeninteressen verhindern, ist die Größe und Zusammensetzung des Verwaltungsrates. Einzelne Gruppeninteressen können keine Dominanz erlangen, die KfW kann eine kontinuierliche Förderpolitik machen.

\subsubsection{Finnvera - Finnland}

Finnvera ist eine spezialisierte Finanzierungsgesellschaft im Besitz des Staates Finnland und gleichzeitig die offizielle finnische Exportkreditagentur (ECA). Finnvera bietet einerseits Finanzierungsleistungen für die Gründung, das Wachstum und die Internationalisierung von Unternehmen und andererseits Garantien für politische oder kommerzielle Risiken aus Exportgeschäften. Finnveras Aufgabe ist es, über die Stärkung der Wettbewerbsfähigkeit der finnischen Unternehmen durch die Vergabe von Darlehen, Inlandsgarantien, Exportkreditgarantien und weiteren Dienstleistungen (etwa im Zusammenhang mit der Finanzierung von Exporten), direkt zur finnischen Wirtschaftspolitik beizutragen. Die entsprechenden finanziellen Risiken werden zwischen Finnvera und anderen Finanzierungsanbietern geteilt, d.h. Finnvera stockt in erster Linie die Angebote kommerzieller Banken und anderer Finanzdienstleister auf.

Finnvera hatte Ende 201627.712 KundInnen (81\% sind KMU mit weniger als 10 Beschäftigten, $11 \%$ andere KMU und Mittelstandsunternehmen sowie im Bereich der Exportgarantien auch Großunternehmen (0,4\%)). 2016 betrug die Gesamtsumme der angebotenen Finanzierungen $€ 5,3$ Mrd. Dabei entfielen $€ 963$ Mio. auf das Geschäftsfeld 
Darlehen, Inlandsgarantien und Exportgarantien sowie $€$ 4,3 Mrd. auf Exportkreditgarantien. Die entsprechende Mittelbindung betrug $€ 20,7$ Mrd. Finnvera verfügt über $376 \mathrm{Be}-$ schäftigte und 15 regionale Büros.

\section{Wesentliche Aspekte der Governance}

Finnveras Tätigkeiten werden durch die politisch festgelegten Ziele der finnischen Industriepolitik sowie anderer relevanter Politikbereiche gesteuert. Zu diesen Zielen gehören: Erhöhung der Zahl der Unternehmensgründungen; (Ermöglichung der) Finanzierung der notwendigen Reaktion von KMU auf entsprechende makroökonomische und sonstige Veränderungen und Förderung von Unternehmenswachstum, Internationalisierung und Exporttätigkeiten. Finnvera muss seine Tätigkeiten am Prinzip ökonomischer Nachhaltigkeit und finanzieller Unabhängigkeit (der Staat deckt einige der Kredit- und Garantieverluste von Finnvera ab, um es Finnvera zu ermöglichen, mehr Risiko einzugehen, als die Geschäftsbanken im Allgemeinen akzeptieren würden) ausrichten.

Finnvera ist ein eigenständiges Unternehmen, das sich zu 100\% im Staatsbesitz befindet. Wesentlich für die Frage nach der Governance sind drei Elemente: (1) Finnvera ist im Rahmen der definierten Aufgaben und der vorgegebenen Zielsetzungen autonom (abgesehen von den nationalen und internationalen Regeln für Banken) und operiert wie ein börsennotiertes Unternehmen vis-à-vis dem Eigentümer (vertreten durch das finnische Wirtschafts- und Arbeitsministerium). (2) Finnvera ist finanziell unabhängig, d.h. die benötigten finanziellen Mittel werden von Finnvera entweder selbst erwirtschaftet oder an den Finanzmärkten eingeworben. (3) Die Steuerung durch das Ministerium ist im Wesentlichen auf die im gemeinsam ausgehandelten Mehrjahresprogramm beschränkt. Letzteres ist insbesondere auf die Reaktionen auf die 2012 veröffentlichte Evaluierung zurückzuführen, in der dem Ministerium eine zu stark operative Steuerung zugeschrieben wurde (Heinonen et al. 2012).

Langfristig betrachtet, muss Finnvera seine Betriebskosten und Ausfälle aus dem eigenen Ergebnis decken, was über einen Zeitraum von 11 Jahren gelungen ist (die Exportbezogenen Aktivitäten sind seit Gründung von Finnvera vor 18 Jahren kostendeckend). Finnvera kann Überschüsse ohne Rücksprache reinvestieren. Es gibt allerdings jährliche Treffen mit dem Eigentümervertreter, die grundsätzlich die dafür geeigneten Themen und Instrumente diskutieren und festlegen.

Finnveras Kommunikation mit dem Eigentümervertreter (Wirtschafts- und Arbeitsministerium) ist im Wesentlichen auf gegenseitigen Informationsaustausch fokussiert, wobei Finnvera halbjährlich die aktuellen Geschäftszahlen liefert. 
Förder- bzw. Finanzierungsentscheidungen werden unabhängig auf der Basis der Mehrjahresprogramme sowie der vereinbarten Regeln und Prinzipien getroffen. Große Förderfälle werden vom Aufsichtsrat direkt entschieden. Der Aufsichtsrat besteht aus sechs bis neun Mitgliedern, die überwiegend aus der Industrie und dem Banken-/Versicherungssektor kommen. Der Aufsichtsrat entscheidet außerdem über die Prinzipien des unternehmenseigenen Risikomanagements, Finnveras Gesamtstrategie sowie zentrale Personalfragen (insbesondere Bestellung und Entlassung der/s Geschäftsführers/in).

\section{Ergebnisse im Überblick: Autonomiegrade}

\section{Operationale Autonomie}

Die fachliche Expertise im Bereich der verantworteten Themen liegt bei Finnvera und dementsprechend erfolgt dort auch die Entwicklung von neuen Instrumenten (z.B. Startup Garantie) und Maßnahmen auf der Basis der eigenen strategischen Intelligenz. Die Vorgaben des Eigentümers mit Bezug zu den Leistungen und Aufgaben sind gering und werden mit Finnvera über den Aufsichtsrat abgestimmt. Als zentrales Element der Steuerung bleibt dem Eigentümer die alleinige Kontrolle über die Vergabe von entsprechenden Evaluationen. Die insgesamt sehr ausgeprägte Autonomie mit Bezug zur eigentlichen Geschäftstätigkeit der Finnvera basiert einerseits auf Vertrauen und dem nachgewiesenen Erfolg ihrer Arbeit (Zitat aus dem Interview: "Success breeds freedom breeds success").

Abbildung 33: $\quad$ Operationale Autonomie der Finnvera

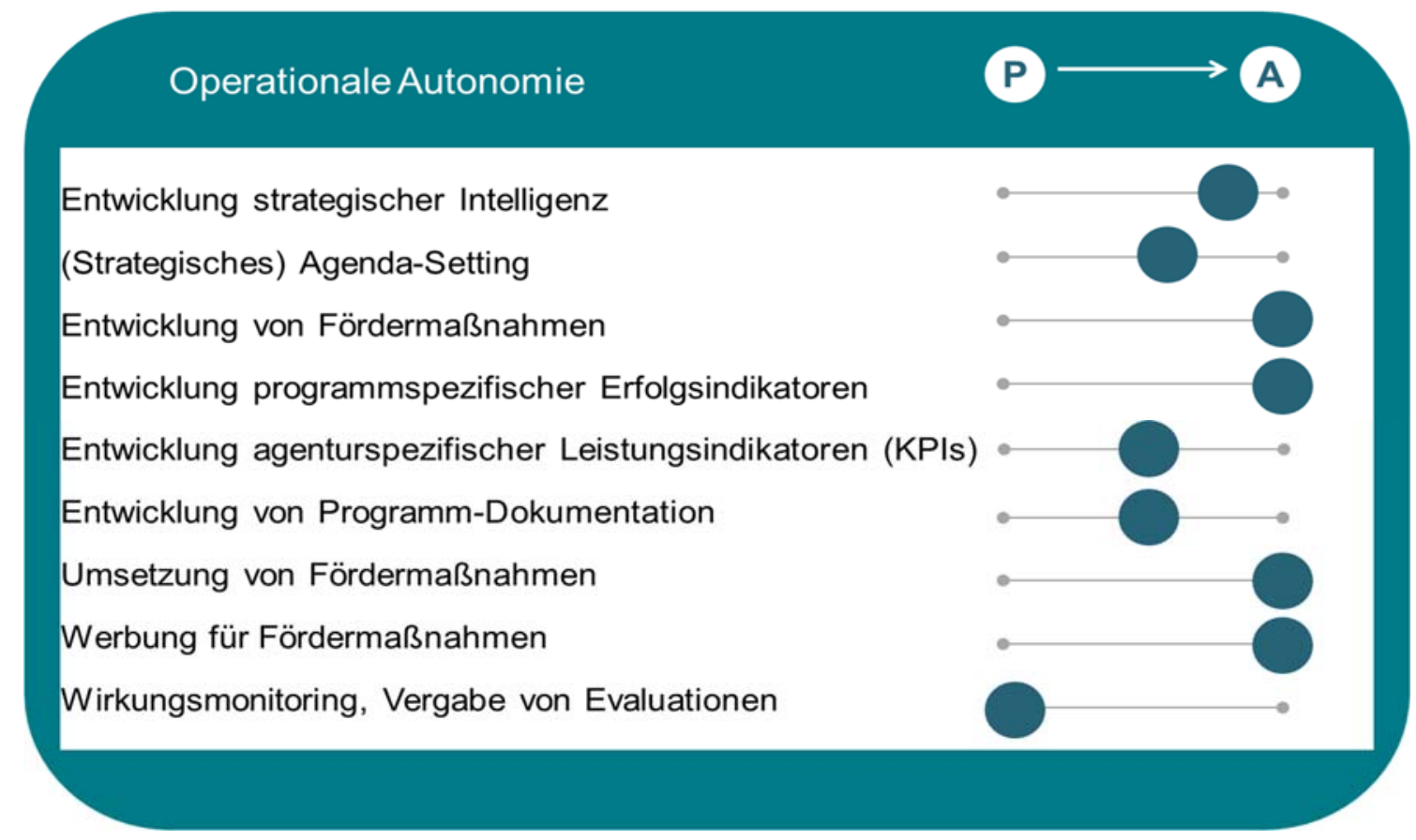




\section{Finanzielle Autonomie}

Finnvera ist grundsätzlich finanziell eigenständig, wobei für bestimmte Förderungen ein Teil der Garantien vom Staat übernommen werden, um eine stärkere Risikoorientierung zu ermöglichen. Die Deckung der Ausgaben und Verluste durch Finnveras Geschäftstätigkeit mit eigenen Mitteln (entweder aus Gewinn oder Re-Finanzierung) war als langfristiges Ziel bei der Schaffung von Finnvera definiert worden und ist inzwischen erreicht. Finnvera kann Überschüsse ohne Rücksprache mit dem Eigentümer reinvestieren. Es gibt allerdings jährliche Treffen, in denen Agentur und Eigentümer die grundsätzlich die dafür geeigneten Themen und Instrumente diskutieren und festlegen.

Abbildung 34: $\quad$ Finanzielle Autonomie der Finnvera

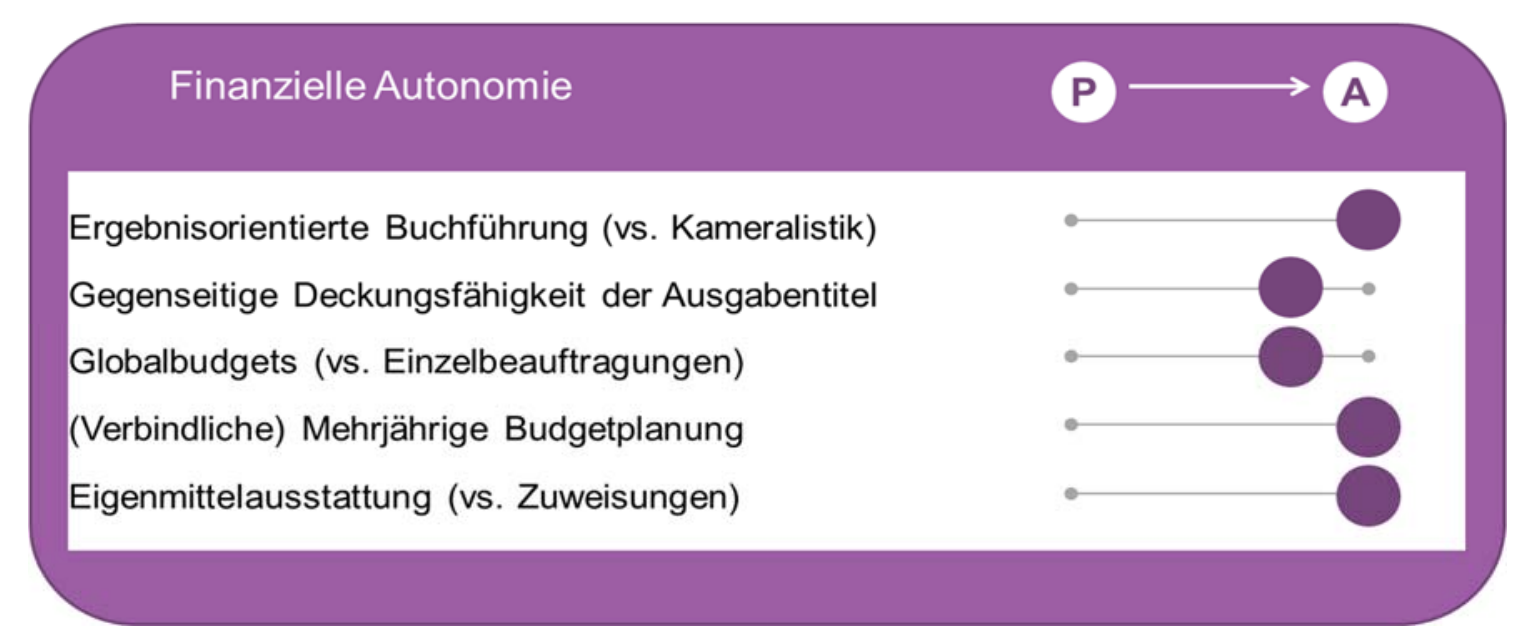

\section{Personelle Autonomie}

Die personelle Autonomie ist im Unterschied zu den beiden anderen davor diskutierten Dimensionen weniger eindeutig geregelt. Hier spielt insbesondere der Aufsichtsrat der Finnvera mit seinen weitreichenden Befugnissen eine Rolle. Während die Mitglieder direkt von der Politik bestimmt werden, so ist der Einschätzung der Interviewpartner zufolge nicht von einer wie auch immer gearteten Einflussnahme bzw. politischen Abhängigkeit auszugehen. Insbesondere die Verortung der Mitglieder des Aufsichtsrats als Vertreter vor allem der finnischen Wirtschaft bzw. Finanzwirtschaft dürfte die tatsächlichen Machtverhältnisse in Richtung Agentur verschieben. Allerdings sieht man bei Finnvera keine unterschiedlichen Einstellungen in Ressort und Agentur, so dass der Effekt selbst einer Verschiebung in Richtung Ministerium keinen direkten Einfluss haben sollte. Ohnedies wird Vertrauen als Basis der Kooperation betont. 
Abbildung 35: $\quad$ Personelle Autonomie der Finnvera

Personelle Autonomie und Kontrollorgane
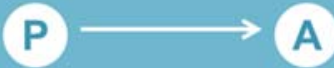

Besetzung des Aufsichtsrates

- Tatsächliche Machtverhältnisse im Aufsichtsrat

Auswahl der GeschäftsführerInnen (m. Weisungsrecht)

Personalpolitik

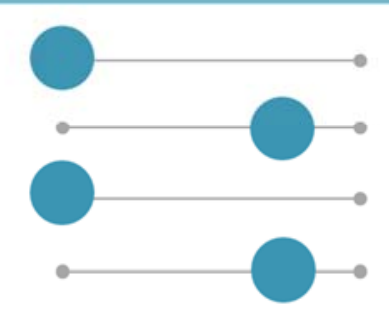

\section{Organisatorische Autonomie}

Die organisationale Autonomie ist durch die eigene Rechtspersönlichkeit gegeben. Auch die Gestaltung der Aufbau- und Ablauforganisation liegen in den Händen der Finnvera.

\section{Qualität der Zusammenarbeit}

Das Verhältnis von Ministerium und Finnvera ist, obgleich eine vertrauensvolle Zusammenarbeit existiert, weniger kooperativ angelegt als vielmehr einer nahezu klassischen Shareholder-Unternehmen-Beziehung ähnlich. Finnvera ist in seiner Tätigkeit nahezu vollkommen unabhängig und versteht sich (und arbeitet) als wäre es ein börsennotiertes Unternehmen (dessen einziger Shareholder der Staat ist) mit den entsprechenden Mechanismen des Reportings von Quartalszahlen usw. Die Strukturen, die etwa moral hazard verhindern, sind letztlich auf die Bestimmung des sehr mächtigen Aufsichtsrats, der Geschäftsführung sowie die Vergabe von Evaluationen beschränkt. Insbesondere letzteres dürfte, der Wirkungsmächtigkeit der erst 2012 erfolgten Evaluierung der gesamten Organisation zufolge, als starkes Steuerungsinstrument ausreichen, um sowohl die prinzipielle Kontrolle über Finnvera zu gewährleisten als auch dem ansonsten auf Vertrauen beruhenden Verhältnis zugrunde liegen. 


\subsection{Vergleich mit FFG}

\subsubsection{VINNOVA - Schweden ${ }^{19}$}

Die Gründung von VINNOVA (The Swedish Governmental Agency for Innovation Systems) im Jahr 2001 als die wichtigste schwedische Förderagentur für Innovationen (d.h. es gibt weitere, themenfokussierte Agenturen) basierte auf einem Zusammenschluss aus drei Vorgängerorganisationen (die technology division of the Swedish Agency for Industrial and Technical Development (NUTEK), die Swedish Agency for Transport Research und Teile der Agency for Work Organisation), die im Auftrag des Ministeriums für Wirtschaft und Innovation tätig ist.

Die inhaltlichen Schwerpunkte basieren auf der Mission: "to promote sustainable economic growth by financing needs-driven R\&D and developing new innovation systems". Anfangs fokussierte VINNOVA hauptsächlich auf die Stärkung von Kooperationen zwischen Unternehmen, dem Forschungs- sowie dem öffentlichen Sektor. Über die Zeit hat sich dies insofern geändert, als sektoral ausgerichtete Programme durch thematische, querliegende sowie an den großen Herausforderungen für Schweden orientierte Programme ersetzt bzw. ergänzt wurden, indem der Ansatz der 'challenge driven innovation' exploriert wurde. Darüber hinaus tritt VINNOVA's strategischer Teil als Finanzier von Studien zur Analyse und Weiterentwicklung des nationalen Innovationssystems auf, da der systemische Fokus der Agenturtätigkeit explizit in dessen Mission enthalten ist.

Die jährlichen Budgets beliefen sich zu Beginn auf rd. 100 Mio. Euro, und stiegen in den letzten Jahren auf rd. 350 Mio. Euro, bei etwa zweihundert Beschäftigten. Rd. 45\% des Budgets ergeht an Universitäten und Forschungsorganisationen, rd. 30\% direkt an Unternehmen; von letzterem rd. 2/3 für KMU.

Die interne Organisation war nach inhaltlichen Schwerpunktbereichen (Biotech, ICT, Manufacturing, etc.) sowie Instrumenten (Clusters \& networks, SME R\&D, Commercialization etc.) organisiert. 2011 erfolgte eine interne Reorganisation der Aufbauorganisation um die elf neuen strategischen Aktivitätsfelder auch in der Organisationstruktur zu reflektieren (zusammengefasst in den drei übergreifenden Feldern: strategically important knowledge areas, innovativeness of specific target groups, cross-border co-operation), wobei VINNOVA einen Schritt in Richtung der Unterstützung der Bildung eines 'innovativen Milieus' vorgenommen hat, jenseits von alleiniger Unterstützung von FuE. Neben dem Hauptsitz in Stockholm gibt es Repräsentanzen in Brüssel und Silicon Valley.

19 Abgesehen von den Interviews basiert die Fallstudie auf: OECD (2015), VINNOVA (2008) und VINNOVA (2012). 


\section{Wesentliche Aspekte der Governance}

Die Budgets werden in der Research Bill auf vier Jahre im Vorhinein abgesteckt, das federführend vom Wissenschaftsministerium, in Abstimmung mit dem Wirtschaftsministerium (und VINNOVA) und allen anderen Ministerien, erstellt und im Parlament eingebracht wird. Der grobe Vierjahreszeitraum wird dann vom Wirtschaftsministerium jährlich für das kommende Jahr spezifiziert. Die jährliche Abstimmung erfolgt dermaßen, dass sich insgesamt sechs Personen aus VINNOVA und dem Wirtschaftsministerium im Frühjahr zu einem jährlichen Reporting Treffen zusammenfinden, und auf Basis dessen im Herbst ein Auftragsschreiben für das Folgejahr formuliert wird. Während es am Anfang der 2000er Jahre durchaus detaillierte Vorgaben (mit 13 Unterzielen) durch den Prinzipal gegeben hatte 20 , beschränkt sich die Beauftragung inzwischen auf einen zweiseitigen Brief, der eine grobe Leitlinie, z.B. für die Budgetverteilung auf Themen, sowie die Berichtspflichten vorgibt. Von beiden Seiten wird das inzwischen erworbene Vertrauensverhältnis betont, das keine detailliertere Vorgehensweise für nötig erscheinen ließe. Im Zuge dessen wird ebenfalls betont, dass zur Prüfung der ordnungsgemäßen Abwicklung ohnehin das Swedish National Audit Office sowie die Swedish National Financial Management Authority zuständig seien.

Die letzte OECD Review der schwedischen Innovationspolitik (OECD 2016) merkt an, dass die schwedische Regierung bislang keine starke Priorisierung von bestimmten Themen vorgibt, sondern diese strategische Aufgabe an die Agenturen, und hier insbesondere VINNOVA, weitergibt. Damit koordiniert VINNOVA die Erarbeitung der strategischen Forschungsagenden indem eine möglichst breite Beteiligung von Stakeholdern einbezogen wird. Diese werden von VINNOVA aufgefordert strategische Roadmaps unter Einbezug von Unternehmen, Universitäten und anderer Stakeholder zu erarbeiten. VINNOVA entscheidet dann auf Basis konkurrierender Roadmaps, welche davon wie unterstützt werden und welche Ziele angestrebt werden. Dies steht im leichten Kontrast zur Selbstdarstellung durch das Ministerium.

\section{Ergebnisse im Überblick: Autonomiegrade}

\section{Operationale Autonomie}

Das schwedischen Wirtschaftsministerium, wie auch die anderen Ministerien, sind relativ klein, dem relativ große und autonome Agenturen gegenüberstehen. VINNOVA stellt hier keine Ausnahme dar. Die Governance beschränkt sich dementsprechend auf grobe

20 Dies war im starken Kontrast zu den Vorgaben der Wissenschaftsagentur, aber auch der Schwesteragentur in Finnland (TEKES). 
Vorgaben und basiert auf einem über die Jahre erworbenen hohen Vertrauen. Dies bedeutet im Konkreten, dass Politik- und Instrumentendesign, und auch die Entwicklung der strategischen Intelligenz inklusive der Vergabe von Evaluierungen zu einem hohen Ausmaß in der Agentur angesiedelt sind. Im Wirtschaftsministerium ist eine einzelne Person mit der kontinuierlichen Abstimmung der VINNOVA Agenden beauftragt. Wenn also eine Abteilung aus dem Ministerium oder ein anderes Ministerium VINNOVA beauftragen möchte, ist diese zentrale Stelle zur Koordination heranzuziehen. Im Interview mit dem Ministerium wurde betont, dass dies nach wie vor eine Herausforderung darstellt, aber im Grunde genommen doch relativ gut funktioniert.

Ein Spezifikum des Schwedischen Rechtssystems wurde von einem Interviewpartner folgendermaßen formuliert: "The Government has quite substantial scope for steering the operations of government agencies. However, it has no powers to intervene in an agency's decisions in specific matters relating to the application of the law or the due exercise of its authority. In many other countries, it is common for an individual minister to have the power to intervene directly through a decision in an agency's day-to-day operations. This possibility does not exist in Sweden, however. Collective Government decision-making and the ban on instructing agencies on individual matters are expressions of the prohibition of 'ministerial rule', as it is often called. The Riksdag [Nationalrat] is responsible for monitoring to ensure that ministerial rule does not occur. Should the Government consider that an agency has not applied a law correctly its only remedy is to seek to amend the relevant legislation."

Abbildung 36: Operationale Autonomie der VINNOVA

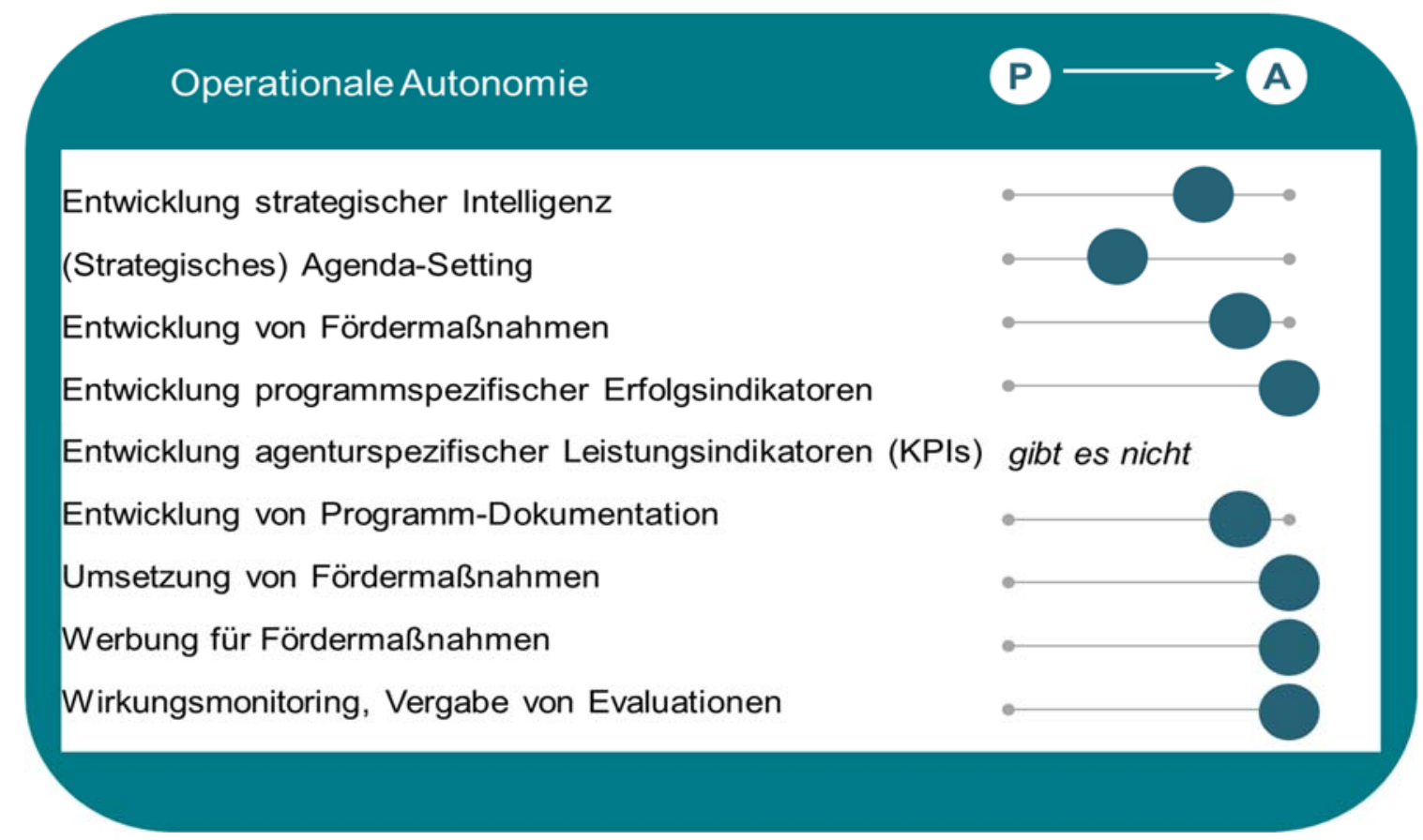




\section{Finanzielle Autonomie}

Die finanzielle Gebarung basiert auf einem Globalbudget, das manchmal hinsichtlich einzelner Themen ('challenge driven innovation') einen Anteil als grobe Orientierung vorgibt, bzw. in Ausnahmefällen wie z.B. der letzten Finanzkrise auch politische Direktiven enthalten kann, dass eine betroffenen Branche besonders unterstützt werden soll. Auf Basis der auf vier Jahre gültigen Research Bill erhält VINNOVA den oben bereits erwähnten 'letter of instruction', der das jeweilige Budget für das Folgejahr spezifiziert. Zusätzliche, unterjährige Beauftragungen sind immer noch möglich, falls dies für nötig erachtet wird. Innerhalb dieser Rahmenbedingungen kann VINNOVA stark autonom agieren. Eine gegenseitige Deckungsfähigkeit der Ausgabentitel ist meist gegeben, kann aber in manchen Fällen, z.B. bei zusätzlichen Beauftragungen nicht ausgeschöpftes Budget auch zurückverlangt werden.

Abbildung 37: $\quad$ Finanzielle Autonomie der VINNOVA

Finanzielle Autonomie

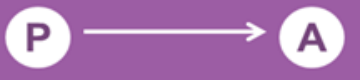

Ergebnisorientierte Buchführung (vs. Kameralistik)

Gegenseitige Deckungsfähigkeit der Ausgabentitel

Globalbudgets (vs. Einzelbeauftragungen)

(Verbindliche) Mehrjährige Budgetplanung

Eigenmittelausstattung (vs. Zuweisungen)

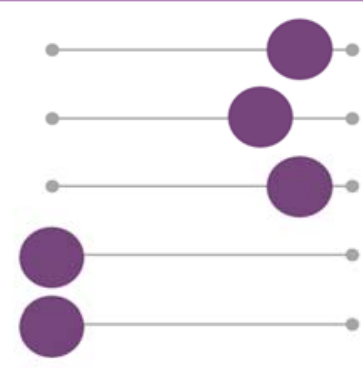

\section{Personelle Autonomie}

Die Personalpolitik basiert auf einer hohe Durchdringung an Personen mit akademischem Hintergrund, und einem ausgewogenen Verhältnis von Beschäftigten direkt von Forschungsinstitutionen sowie mit unternehmerischem Hintergrund. Es werden auch regelmäßig Praktika für Universitätsangehörige angeboten, sowie auch Austauschprogramme mit anderen Innovationsagenturen (insb. TEKES) umgesetzt, um eine offene Lernumgebung aufrecht zu erhalten.

Im siebenköpfigen Aufsichtsrat sind überwiegend VertreterInnen der Industrie, neben AkademikerInnen und Sozialpartnern vertreten, die von der Regierung ernannt werden. Aufgrund der Konzeption hat der Aufsichtsrat ein sehr hohes Ausmaß an Kontrolle über 
die Agentur - in der Praxis liegt die Macht jedoch sehr stark beim Chief Executive Officer (CEO) von VINNOVA, der derzeit einen industriellen Hintergrund aufweist.

Abbildung 38: $\quad$ Personelle Autonomie der VINNOVA

Personelle Autonomie und Kontrollorgane

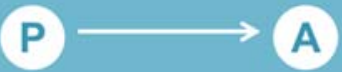

Besetzung des Aufsichtsrates

- Tatsächliche Machtverhältnisse im Aufsichtsrat

Auswahl der Geschäftsführerlnnen (m. Weisungsrecht)

Personalpolitik

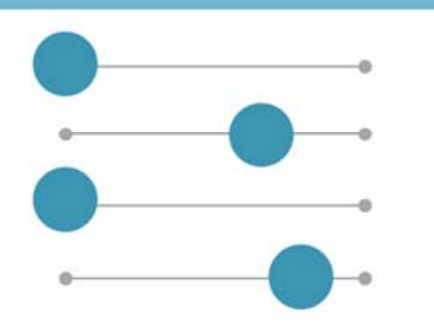

\section{Organisatorische Autonomie}

VINNOVA ist eine eigene Rechtspersönlichkeit im Eigentum der schwedischen Regierung, und kann folglich Prozesse und Strukturen innerhalb der Agentur selbst bestimmen. Eine eigene Strategieabteilung erarbeitet Vorschläge und vergibt Studien und Evaluierungen. Während die externen Evaluierungen teilweise einen betont langfristigen Charakter mit systemischer Perspektive aufweisen, wird ein internes Wirkungsmonitoring für kurzfristige Portfoliobeobachtungen verwendet, um Entscheidungen über die Performance von (neuen) Initiativen zu unterstützen.

\section{Qualität der Zusammenarbeit}

Das enge Verhältnis von relativ wenigen Personen im Ministerium und VINNOVA schaffte über die Jahre ein besonderes Vertrauensverhältnis. Das jährliche Reporting und die Diskussion über das kommende Jahr werden im engsten Kreis umgesetzt. Im Wirtschaftsministerium waren rd. 20 Personen in weitere Diskussionen eingebunden. VINNOVA hat jedoch in letzter Zeit den Kreis von AnsprechpartnerInnen darüber hinaus erweitert, und sieht nun rund 40 Personen quer über mehrere Ministerien und andere Stakeholder als deren Zielpublikum, das sie in den innovationspolitischen Diskussionsprozess aktiv involviert, um eine breitere Abstimmung sicherzustellen.

Der Anreiz für VINNOVA, seine Ziele zu verfolgen und zu erreichen, resultiert aufgrund mehrerer Mechanismen, wobei die formalen Anreize einerseits durch ein vorgegebenes 
jährliches Reporting an den Prinzipal, und andererseits durch die hierarchischen Strukturen über den theoretisch starken Aufsichtsrat sowie über die Bestellung des CEO durch den Prinzipal gegeben ist.

\subsubsection{VLAIO - Flandern, Belgien}

Die Vlaanderen Agentschap Innoveren \& Ondernemen VLAIO ist die staatliche flämische Agentur für Innovation \& Enterpreneurship. Ihr Auftrag ist die Umsetzung der flämischen Wirtschafts-, Innovations- und Industriepolitik, die in Belgien nicht auf der Ebene des Föderalstaats, sondern auf der Ebene der Regionen angesiedelt ist. VLAIO resultierte aus dem Zusammenschluss der Agentur für Wissenschaft und Technologie (iwt) und Enterprise Flanders im Jahr 2016. Die Agentur hat 370 Mitarbeiterinnen.

Die Zielgruppe sind vorwiegend Unternehmen. VLAIO unterstützt flämische Unternehmen in der Gründungs- und Wachstumsphase. Daneben gibt es verschiedene Programme für Kooperationen mit akademischen Einrichtungen. Außerdem agiert VLAIO als Ansprechpartner für ausländische Unternehmen und unterstützt bei der Standortsuche sowie als Manager der europäischen Regionalentwicklungsgelder (ERDF) in Flandern.

Der Jahresumsatz liegt bei 510 Mio. Euro. Diese fließen zum überwiegenden Teil in die Innovationsförderung, bei der Unternehmen FuE-Projektförderung erhalten. Einen kleineren Teil machen Innovationsgutscheine aus. Neben der Innovationsförderung geht auch etwa ein Drittel des Budgets in die Investitionsförderung bzw. in die Unterstützung von Unternehmensgründungen und Unternehmenswachstum. Neben den verschiedenen Zuschussprogrammen macht die VLAIO auch Beratung (z.B. für Gründer sowie für weitere Möglichkeiten der Unternehmensfinanzierung).

Insgesamt dient die Fusion vor allem der Einrichtung eines one-stop-shops für Unternehmen und der Schwerpunkt liegt nun auf innovativen Unternehmen.

\section{Wesentliche Aspekte der Governance}

VLAIO ist direkt an das Flämische Ministerium für Arbeit, Wirtschaft, Innovation und Sport angebunden. Die Vorgängerorganisationen waren organisatorisch sehr unterschiedlich aufgestellt: die iwt (die heutigen Innovationsprogramme) war eine eigenständige von der Regierung beauftragte Agentur, während Enterprise Flanders (die heutigen Investitionsprogramme) nicht unabhängig, sondern Teil der Regierung war. Aus diesen sehr unterschiedlichen Startbedingungen entstand ein "hybrides" Konstrukt: VLAIO bezeichnet sich selbst als "interne" Agentur, die nicht vertraglich beauftragt, sondern hierarchisch direkt dem Ministerium unterstellt ist. Gleichzeitig erhält VLAIO jährlich sein 
Budget von der Regierung zugewiesen, das die Agentur für einen Teil der Programme (Innovationsprogramme) in einem Fonds selbst verwaltet, so dass die Förderabwicklung in diesem Bereich schnell und flexibel stattfinden kann.

VLAIO entwickelt einen jährlichen Geschäftsplan, welche Programme und internen Projekte es angehen will. Dazu gehört auch der Vorschlag für ein Budget. Feinheiten des Geschäftsplans handelt VLAIO mit dem Innovationsministerium aus, eventuelle Modifikationen am Budget mit dem Finanzministerium. Hat die Regierung den Plan abgenommen, geht er ins Parlament zur endgültigen Abstimmung. Im laufenden Jahr ist das Budget meist im Mai weitgehend klar und oft erst im Oktober endgültig entschieden. Eine mehrjährige strategische Planung gibt es nicht, die Richtung der Agentur ergibt sich unmittelbar aus den politischen Gegebenheiten. Unterjährige Anpassungen im Falle von Initiativen des Ministeriums sind möglich und gehen in manchen Fällen neuer Programme auch mit Budgetaufstockungen einher. Die Agentur kann im Budget innerhalb thematischer Programmgruppen Gelder nach Bedarf verschieben.

Das Ministerium hat direkte Zugangsmöglichkeit zu den Geschäftszahlen von VLAIO, zudem berichtet die Agentur regelmäßig und es gibt regelmäßige, häufige Audits. Es kommen keine KPIs zur Steuerung der Agentur zum Einsatz, es erfolgt eher eine qualitative informelle Beurteilung, ob der Jahresplan gut umgesetzt wurde.

\section{Ergebnisse im Überblick: Autonomiegrade}

\section{Operationale Autonomie}

Die "hybride" Organisation wird in der Programmabwicklung deutlich. Hier sind die beiden Modelle der Vorläuferorganisationen (bisher) erhalten geblieben. Die Innovationsprogramme (die früheren Programme des iwt) werden weitgehend autonom durchgeführt. Es gibt ein Entscheidungskomitee, das auf der Basis der Einschätzungen unabhängiger ExpertInnen die Förderentscheidungen trifft. Dieses Gremium ist wie ein Aufsichtsrat besetzt, in dem Sozialpartner, Industrie, Wissenschaftseinrichtungen sowie Regierungsvertreterlnnen und weitere von der Regierung benannte Personen sitzen. Aufgrund der vielen verschiedenen Sichtweisen agiert das Gremium frei von politischem Einfluss. Dagegen werden die Gründungs- und Wachstumsförderungen ähnlich vergeben, wie dies bei Enterprise Flanders der Fall war. VLAIO bereitet auf der Basis externer Begutachtungsverfahren Förderentscheidungen vor, die das Ministerium abzeichnet.

Die Entwicklung neuer Förderinitiativen erfolgt in der Agentur. Die Initiative hierfür erfolgt im Rahmen des jährlichen Geschäftsplans durch die Agentur oder unterjährig durch das Ministerium. 
Das interne Monitoring der Förderprogramme liegt bei der Agentur und muss derzeit noch aufgebaut werden. Auch die Vergabe von Wirkungsanalysen liegt bei der Agentur.

Abbildung 39: $\quad$ Operationale Autonomie der VLAIO

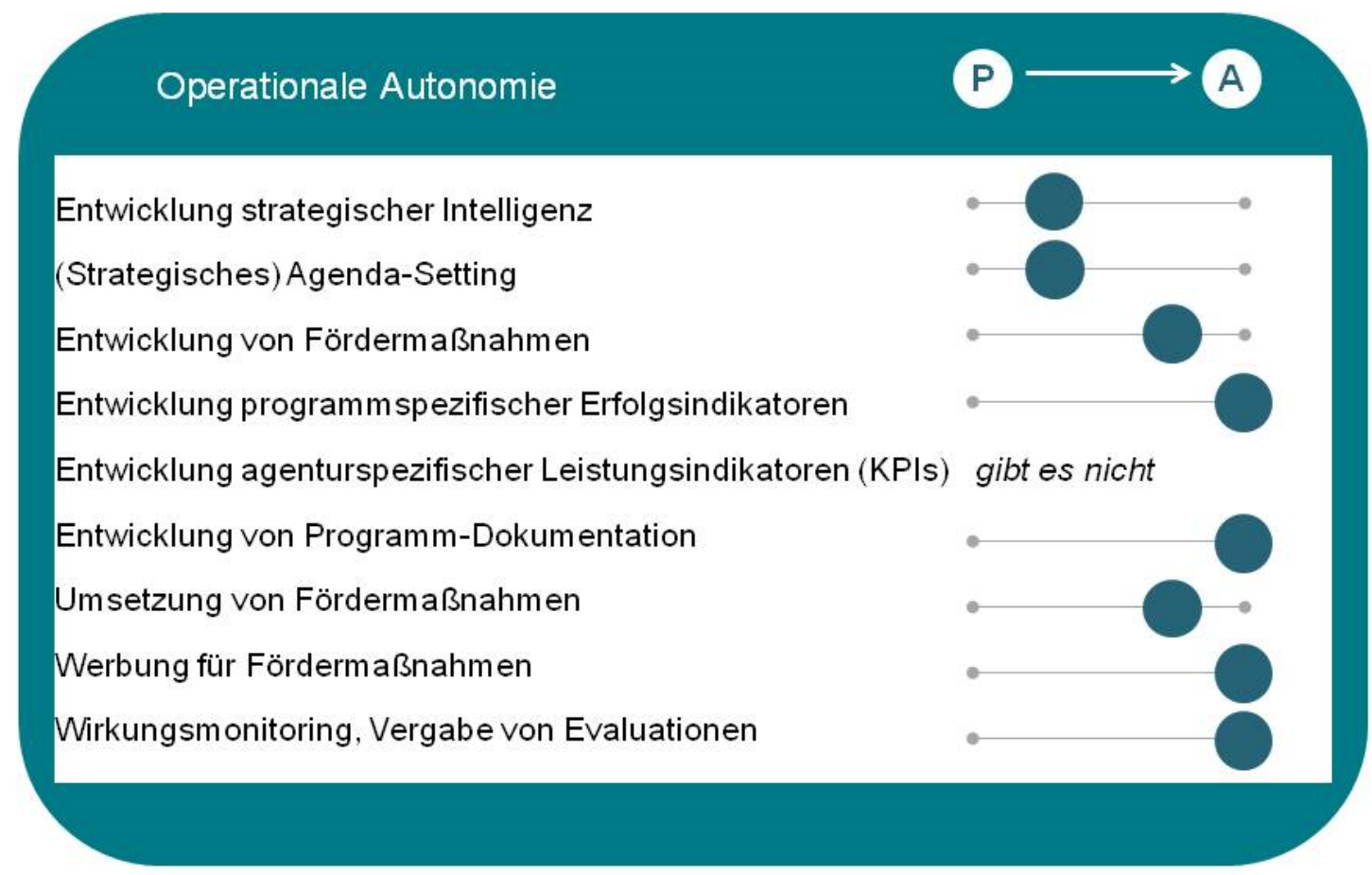

\section{Finanzielle Autonomie}

(vgl. dazu die Ausführungen oben unter "Wesentliche Aspekte der Governance")

Abbildung 40: $\quad$ Finanzielle Autonomie der VLAIO

\section{Finanzielle Autonomie}

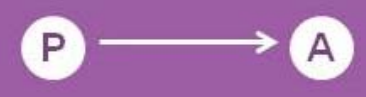

Ergebnisorientierte Buchführung (vs. Kameralistik)

Gegenseitige Deckungsfähigkeit der Ausgabentitel

Globalbudgets (vs. Einzelbeauftragungen)

(Verbindliche) Mehrjährige Budgetplanung

Eigenmittelausstattung (vs. Zuweisungen)

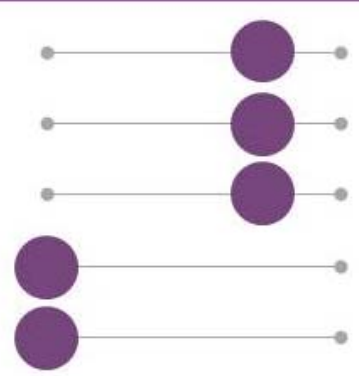




\section{Personelle Autonomie}

Das Entscheidungskomitee der Innovationsprogramme entspricht dem ehemaligen Aufsichtsrat der iwt und hat bei VLAIO für die Innovationsprogramme nun dieselbe Funktion. Zwar entsenden sowohl das Finanz- als auch das Innovationsministerium Mitglieder und das Innovationsministerium benennt darüber hinaus noch einige Persönlichkeiten, dennoch sind weitgestreute Interessen im Gremium vorhanden, da die Sozialpartner VertreterInnen entsenden dürfen und außerdem die Industrie und die Hochschulbildung und Wissenschaft vertreten ist.

Der Stellenplan für VLAIO ist an den Stellenplan des Ministeriums gekoppelt, aber die Besetzung von Stellen kann autonom erfolgen. Die Agenturleitung wird vom Ministerium besetzt.

Abbildung 41: $\quad$ Personelle Autonomie der VLAIO

\section{Personelle Autonomie}

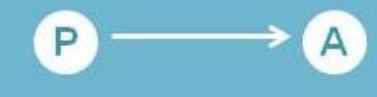

Besetzung des Aufsichtsrates

- Tatsächliche Machtverhältnisse im Aufsichtsrat

Auswahl der Geschäftsführerlnnen ( $\mathrm{m}$. Weisungsrecht)

Personalpolitik

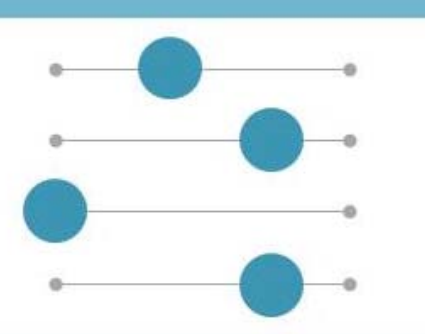

\section{Organisationale Autonomie}

Als Teil des Ministeriums hat VLAIO keine eigene Rechtspersönlichkeit, kann aber Teile seines Budgets selbst verwalten (siehe oben). Aufbau- und Ablauforganisation sind derzeit von den beiden Vorgängerorganisationen übernommen, eine Überarbeitung mit dem Ziel einer größeren Vereinheitlichung ist im Gange.

\section{Qualität der Zusammenarbeit}

Der Anreiz für VLAIO, seine Ziele zu erreichen, ergibt sich aus dem hierarchischen Verhältnis und den Kontrollmöglichkeiten des Ministeriums. Gleichzeitig teilen die Beteiligten die Ansicht, dass Förderungen schnell und flexibel erfolgen sollten und die Entscheidungen Objektivität brauchen, um bei der Industrie Akzeptanz zu finden. Daher schuf das Ministerium trotz der engen Anbindung VLAIO als unabhängigen Geschäftsbereich. 
Im Augenblick findet zudem ein Lernprozess statt, in dem deutlich wird, dass die Abwicklung der Innovationsprogramme diese Anforderungen besser erfüllen kann als die Abwicklung der Investitionsprogramme. Die an das Entscheidungskomitee übertragenen Förderentscheidungen für die Innovationsprogramme führen zu qualitativ hochwertigen und objektiven Ergebnissen und genießen den Ruf der politischen Unabhängigkeit. 


\section{$9 \quad$ Zukünftige Optionen}

\section{$9.1 \quad$ Einleitung und Zielsetzung}

In Österreich nutzen der Bund bzw. die Bundesregierung rechtlich unabhängige Organisationen (Agenturen) für die Umsetzung und auch Gestaltung wesentlicher Aspekte der Förderpolitik in den Politikfeldern Forschungs-, Innovations-, Technologie- und Wirtschaftspolitik. Unabhängig von der Frage, ob diese Entwicklung und insbesondere die Schaffung der zwei untersuchten Agenturen FFG und aws im Zuge der Strukturreform streng genommen dem Konzept des New Public Management oder einer traditionellen Nutzung von Intermediären im Prozess der Politikvermittlung und -umsetzung entspricht, wurde damit die Erfüllung von insgesamt vier wesentlichen Zielen verknüpft. Dazu zählen die Reduktion organisatorischer und inhaltlicher Komplexität, die Vermeidung immanenter Koordinationsschwierigkeiten, die verbesserte Steuerungsfähigkeit gegenüber den Zielgruppen sowie die Verbesserung der Umsetzungschancen von Regierungszielen. Vor dem Hintergrund der entsprechenden Erkenntnisse und Bewertungen des Status quo sowie internationaler Beispiele besonders erfolgreicher Systeme und Konstellationen (siehe Kapitel 8) werden in diesem Kapitel drei Zukunftsoptionen für Österreich auch hinsichtlich der jeweiligen Vor- und Nachteile diskutiert: die Weiterführung des Status quo, die Weiterentwicklung des Status quo und schließlich die vollständige Agencification mit voller Agenturautonomie und unter Einsatz von Instrumenten einer Globalsteuerung.

Für die Beschreibung der möglichen Zukunftsoptionen ist zu beachten, dass eine inhaltliche Ausrichtung der Förderportfolios explizit nicht Gegenstand der Evaluierung war. Vielmehr fokussierte diese auf die Organisationen FFG und aws, d.h. deren interne Funktionalität, um ihre Mission umsetzen zu können sowie ihre Relation mit ihren Eigentümern und Auftraggebern. Dies sind auch die wesentlichen Dimensionen der möglichen zukünftigen Entwicklungspfade.

Wie in der Einleitung der aws Eigentümerstrategie des bmwfw und bmvit vom Dezember 2014 wiedergegeben, waren die generellen Ziele des Bundes bei der Schaffung von Agenturen "bedarfsgerechtere und wirtschaftlichere Leistungserbringung, Änderung von der Input- zur Outputorientierung, Erhöhung der Flexibilität für die Leistungserbringung, Schaffung von Kostentransparenz, Entlastung des Bundeshaushaltes, Mobilisierung von gebundenem Bundesvermögen sowie eine erhöhte Markt- und Nachfrageorientierung durch direkten Kontakt. Um diese Ziele zu erreichen, sind den Gesellschaften zum einen mehr Autonomie zu geben und größtmögliche Spielräume einzuräumen" (bmwfw/bmvit 2014). Zusammen mit den Befunden aus der vorliegenden Evaluierung wird damit eine Entwicklungsrichtung für die FFG und die aws vorgezeichnet, die in Folge anhand von drei Zukunftsoptionen interpretiert wird. 


\section{$9.2 \quad$ Hintergrund}

Wie oben beschrieben, muss die Entwicklung von Zukunftsoptionen nicht nur Rücksicht auf die Bedingungen des österreichischen Wirtschafts- und Innovationssystems, sondern auch auf die Tatsache nehmen, dass mit der Schaffung der Agenturen FFG und aws sowie den damit verbundenen Zielen eine gewisse Pfadabhängigkeit, nämlich in Richtung der Aufgabendelegation an rechtlich eigenständige Agenturen bereits vorgegeben ist.

Die Befunde der vorliegenden Evaluierung weisen zudem auf die wesentlichen Herausforderungen hin, die vor allem hinsichtlich der Steuerung und Koordination von Agenturen gegeben sind (OECD 2005, Hammerschmied et al. 2008, Overmann/van Thiel 2016, Overmann 2016). Diese gelten unabhängig von der betrachteten Zukunftsoption weiter und müssen jeweils in Kauf genommen bzw. gelöst werden:

- Eigenständige Agenturen mit (teilweise oder vollständig) an sie delegierten Aufgaben stellen die Eigentümer (Ressorts) vor Schwierigkeiten bezüglich des Umgangs und Einsatzes weicherer Formen von politischer Steuerung als Ersatz für direkte und hierarchische Kontrolle, die unter Umständen zu einer 'Untersteuerung' führen können. ${ }^{21}$

- Agencification kann zu einer Fragmentierung und zum Verlust des Primats der Politik führen, inklusive der Frage nach nachlassender oder nicht mehr gegebener demokratischer Legitimierung.

- Der Fokus auf eine modernere Bürokratie und Verwaltungspraxis und darin insbesondere auf Schnelligkeit und Transparenz von Entscheidungen und ganz generell Prozessen kann den allzu oft nur implizit formulierten Wunsch nach Leistungsverbesserungen bzw. Stärkung der Rechenschaft und Verantwortlichkeit überlagern.

Jede zukünftige Entwicklung muss unabhängig von dem Ausmaß der Veränderung im Vergleich zum Status quo Antworten darauf liefern, wie - auch in Österreich -besonders häufig begangene Fehler vermieden bzw. ihre anhaltenden Effekte ausgeglichen werden können. Dies gilt sowohl für die Phase der Weiterentwicklung als auch für pfaddominierende Entscheidungen und Entwicklungen aus der frühesten Phase der Konzeption und Umsetzung der Prozesse, die zur Schaffung der Agenturen geführt haben:

- zu wenig anspruchsvolle, und damit leicht erreichbare Ziele,

- wichtige Aktivitäten werden von Zielvereinbarungen nicht erfasst,

- Ziele sind so formuliert, dass die Zielerfüllung kaum überprüft werden kann,

21 Der in der vorliegenden Evaluierung zumeist verwendete Begriff der Governance verweist bereits auf ein von hierarchischer Kontrolle zu unterscheidendes Steuerungsverständnis. 
- keinerlei Sanktionen für nicht erfüllte Ziele; zu umfassende und verwässerte Berichte (die aber jedenfalls veröffentlicht werden sollten),

- zu starke Bestimmung der Kontrakte durch die zu steuernde Organisation anstatt durch die - zumindest theoretisch - steuernden Ministerien.

Die entsprechende Entwicklung der unten beschriebenen Optionen folgt dabei mehreren theoretisch und praktisch fundierten Leitgedanken und zentralen Erkenntnissen:

- Sowohl die im Rahmen der vorliegenden Evaluierung durchgeführten Analysen von insgesamt vier internationalen Agenturen als Fallstudien als auch Analysen anderer Autoren zeigen auf, dass kein einzelnes 'best-practice Modell' für eine Förderungsagentur existiert (Glennie/Bound 2016). Die inhaltliche Ausrichtung einer Agentur kann nur vor dem Hintergrund der Ausgestaltung des Wirtschafts- und Innovationssystems insgesamt beurteilt werden.

- Seitens des jeweiligen Prinzipals bedarf es Klarheit darüber, welche Rolle(n) (Aufgaben) eine Agentur im Wirtschafts- und Innovationssystem ganz konkret übernehmen soll und welche konkreten Ziele der Bewertung des politischen Erfolgs bzw. des dahinterliegenden Agenturhandelns damit verbunden sind.

- Die Organisationsstruktur sowie die Governance der Agenturen bedürfen einer gewissen Flexibilität, um auf neue Herausforderungen sich schnell ändernder Strukturen und Bedingungen reagieren zu können. Dazu zählen die Möglichkeit zu selbstständiger, koordinierter Adaption des Agenturportfolios, die Aufnahme von und Orientierung an neuen Zielgruppen sowie die Möglichkeit des Experimentierens mit neuen Ansätzen der Förderung. Damit verbunden ist eine entsprechend entwickelte Fehlerkultur.

- Prinzipal und Agentur müssen inhaltlich und bezüglich der (internen) Koordination stark sein und dahingehend auf Augenhöhe agieren sowie ihre Ressourcen für ihre Kernaufgaben aufwenden. Die Themenhoheit der Prinzipale muss dabei klar gegeben sein und Informationsasymmetrien zwischen Prinzipal und Agentur umfassend abgebaut werden. Aus den Interviews, die für den internationalen Vergleich geführt wurden, kam deutlich heraus, dass dies auf der Basis eines diskursiven Prozesses gelingen kann, indem gegenseitiges Vertrauen mittelfristig aufgebaut wird, das sich durch nachweislich gute Arbeit der Agenturen und einem Verzicht auf Mikro-Management durch die Prinzipale entwickelt.

Es kommt weiters weniger darauf an, die Vorgaben möglichst präzise und genau umzusetzen und Zielabweichungen möglichst hart zu sanktionieren, sondern vielmehr darauf, dass Prozesse der Zielfindung und Zielkorrektur als gemeinsame Lernprozesse auszugestalten sind, in denen gegenseitige Befruchtung stattfindet, die Schnittstellen jedoch klar definiert sind. 


\subsection{Drei Optionen für die Zukunft}

Im Folgenden wird anhand von drei Zukunftsoptionen dargestellt, wie sich die Agenturen und ihr Verhältnis zu ihren Eigentümern in Zukunft gestalten lassen.

- Agencification Stufe 1: Weiterführung des Status quo,

- Agencification Stufe 2: Weiterentwicklung des Status quo,

- Agencification Stufe 3: Höhere Autonomie durch Globalsteuerung.

Die Beschreibung der drei Zukunftsoptionen erfolgt entlang der vier Dimensionen (1) Governance, (2) Finanzierung, (3) Aufgaben und (4) Organisationsstrukturen und Managementprozesse, und veranschaulicht gleichzeitig, welche Konsequenzen damit einhergehen. Hier wird keine taxative Aufzählung von z.B. Aufgaben angestrebt, vielmehr werden jene Themen betont, die eine höhere Relevanz bezüglich der Ausgestaltung von Optionen aufweisen.

Die Beschreibung der Handlungsoptionen gibt Leitlinien vor. Eine Operationalisierung der zu ihrer Umsetzung notwendigen Schritte (z.B. Ausgestaltung der Vertragswerke) sollte im Nachgang der Evaluierung durch die Eigentümerressorts erfolgen.

\subsubsection{Agencification Stufe 1: Weiterführung des Status quo}

Die Fortführung des Status quo im Sinne einer ersten Option für die Zukunft führt zu keinen Veränderungen und dementsprechend alle Stärken und Schwächen des derzeitigen Systems weiter. Obgleich die Weiterschreibung der aktuellen Situation eine Reihe von Nachteilen beinhaltet, nämlich die konsequente Perpetuierung der im vorliegenden Bericht herausgearbeiteten Defizite und Probleme, gibt es auch einen Vorteil: alle Akteure haben für das geltende System expliziter und impliziter Regeln, der Governancemechanismen usw. entsprechende Routinen entwickelt. Diese sichern derzeit und gegebenenfalls auch künftig die grundsätzliche Leistungsfähigkeit der Agenturen und damit auch ihrer Eigentümer, auch wenn diese damit unter ihrem Potenzial bliebe. Es sind damit auch zwei wesentliche Unsicherheitsfaktoren gegeben: zum einen die derzeitig zunehmende Volatilität des Systems in Bezug auf Einfluss und Steuerungsmechanismen, sowie zum anderen die große Abhängigkeit von individuellen bzw. bilateralen Lösungen, die teilweise von einzelnen Personen entwickelt und getragen werden. Verschieben sich die Einflusskanäle weiter z.B. in Richtung Zunahme des Einflusses der Ministerkabinette, und/oder verlassen zentrale Personen das System, ist unklar, wie stabil und leistungsfähig der Status quo bleibt. 
Option 1: $\quad$ Weiterführung des Status quo

\begin{tabular}{|c|c|}
\hline Governance & Finanzierung \\
\hline $\begin{array}{l}\text { Agieren von Prinzipal und Agent auf Basis rele- } \\
\text { vanter Gesetze, der Leitbilder der Ministerien, } \\
\text { der Ziele auf Basis der wirkungsorientierten } \\
\text { Haushaltsführung, der relevanten Richtlinien } \\
\text { und Eigentümerstrategie sowie operativ auf Ba- } \\
\text { sis von wenigen Rahmen-, und zahlreichen Aus- } \\
\text { führungsverträgen (AV) } \\
\text { Koordination per Eigentümer Jour fixes und mit- } \\
\text { tels Quartalsgesprächen } \\
\text { Parallele/überlagerte Aktivitäten zwischen Kabi- } \\
\text { netten und Fachabteilungen } \\
\text { Eigentümerstrategien benennen input-orientierte } \\
\text { Kennzahlen auf Agenturebene, aber kaum Out- } \\
\text { put- und wirkungsorientierte Indikatoren } \\
\text { Evaluierungen und Monitorings werden teilweise } \\
\text { von den Eigentümern beauftragt, teilweise von } \\
\text { der Agentur, Evaluierungsberichte werden nicht } \\
\text { immer veröffentlicht }\end{array}$ & $\begin{array}{l}\text { Absteckung des Agenturbudgets auf Basis } \\
\text { der Mehrjahrespläne (drei Jahre), jährliche } \\
\text { Detailbudgets auf Programmebene } \\
\text { Lange Abstimmungswege bei Budgetzuwei- } \\
\text { sung für hohe Anzahl von Ausführungsver- } \\
\text { trägen } \\
\text { Vollkostenprinzip bei aws, Teilkostenprinzip } \\
\text { bei FFG } \\
\text { Budgets können von Agenturen nicht zwi- } \\
\text { schen Ausführungsverträgen verschoben } \\
\text { werden }\end{array}$ \\
\hline Aufgaben & $\begin{array}{l}\text { Organisationsstrukturen und } \\
\text { Managementprozesse }\end{array}$ \\
\hline $\begin{array}{l}\text { Entwicklung der Förderprogramme teilweise } \\
\text { durch die Agenturen, teilweise durch Ministerien } \\
\text { Zielgruppenansprache und Beratung durch } \\
\text { Agentur; Zielgruppenmobilisierung und Commu- } \\
\text { nity Building bei Missionsorientierung teilweise } \\
\text { durch Ressorts oder andere Organisationen } \\
\text { Entwicklung strategischer Intelligenz nicht klar } \\
\text { geregelt und je nach Engagement der handeln- } \\
\text { den Personen ungleich verteilt }\end{array}$ & $\begin{array}{l}\text { Deutlich höhere Sichtbarkeit und bessere } \\
\text { Positionierung der Agenturen vis-à-vis den } \\
\text { Fördernehmern (im Vergleich zu den Mini- } \\
\text { sterien) } \\
\text { Prozessentwicklung und -optimierung (als } \\
\text { Handlungsmaxime) in den Agenturen }\end{array}$ \\
\hline
\end{tabular}

Die wesentlichen Konsequenzen einer unveränderten Weiterführung des Status quo bestehen zunächst in der potentiellen Verschärfung der Gesamtsituation bei gleichzeitiger Zunahme an Unsicherheit und demzufolge einem Bedeutungsverlust der eingespielten Routinen. Ganz konkret bedeutet dies, dass die Durchgriffsmöglichkeiten der Ressorts zwar prinzipiell unverändert blieben, durch die abnehmende Steuerungsfähigkeit jedoch weiter geschwächt werden könnten. Dies wird dadurch verschärft, dass die zur Verfügung stehenden Governanceinstrumente für eine optimale Steuerung nicht geeignet sind und es keine echte Koordination zwischen den Eigentümerressorts gibt. Damit rückt die Auftraggeberfunktion noch stärker in den Vordergrund, und das System der Einzelbeauftragungen und Ausführungsverträge würde noch mehr Ressourcen auf beiden Seiten binden. Eine Gefahr ist in diesem Zusammenhang auch in einer weiteren Verstär- 
kung der Informationsasymmetrien zwischen Prinzipal und Agentur zu sehen. Die Weiterführung des Status quo würde damit eine bewusste Fortsetzung der zeitlichen Verzögerungen bei Budgetzuweisungen aufgrund der zahlreichen Programme und Ausführungsverträge sowie eine potenzielle Verschlechterung der Zielgruppenansprache und Qualität der Leistungen der beiden Agenturen implizieren, die ihr Potenzial damit nicht vollständig ausschöpfen könnten.

Der zentrale Vorteil der nun folgenden zwei Zukunftsoptionen liegt in dem empirisch auch international feststellbaren Befund (siehe Kapitel 8 sowie z.B. Glennie/Bound 2016), dass eine höhere Flexibilität der Umsetzung in der Agentur zu klarer kommunizierbaren Produkten, eine Beschleunigung von Prozessen zu einer besseren Zielgruppenansprache, und kurze Lernschleifen sowie eine flexible Kombination von Instrumenten zu einer größeren Hebelwirkung der eingesetzten Fördermittel führen.

\subsubsection{Agencification Stufe 2: Weiterentwicklung des Status quo}

Zukunftsoption 2 "Weiterentwicklung des Status quo" fokussiert auf eine Korrektur insbesondere der auf die Governance bezogenen Aspekte in Richtung Stärkung der Ressorts durch eine konsequentere strategische Steuerung der Agenturen, sowie gleichzeitige Stärkung der Agenturen durch eine größere Unabhängigkeit auf der operativen Ebene. Dies entspricht im Wesentlichen der gegenwärtigen Governance der flandrischen VLAIO, die trotz ihrer formellen Zugehörigkeit zum Ministerium eine gewisse Gestaltungsfreiheit über ihr Portfolio besitzt und eine eigene Jahresplanung aufstellt. Die Agenturaufgaben wären mit Ausnahme der Portfoliogestaltung, d.h. der Aufwertung des Instrumentendesigns und größeren Freiheitsgraden bei der Programmentwicklung damit weitgehend wie bisher gestaltet; der Prinzipal würde seine Kontrollaufgaben jedoch stärker wahrnehmen. Eine zentrale Herausforderung besteht allerdings darin, dass für ein derartiges System noch keine Handlungsroutinen existieren, deren Entwicklung zumindest in der Übergangsphase nicht ohne zusätzliche Ressourcen möglich sein wird.

Für das derzeit existierende System gibt es nicht nur solche Routinen, sondern es funktioniert in vielen Bereichen auch durchaus gut und effizient. Daher ist zumindest für diese Bereiche bei den darin wirkenden Personen mit Widerstand gegen eine Veränderung zu rechnen, die hier ja auch als Abkehr vom gut funktionierenden Status quo und zunehmende Unsicherheit interpretiert werden kann. Hinzu kommt, dass die inkrementelle Weiterentwicklung des Status quo die gleichzeitige und nicht durch eine einheitliche Vision oder strategische Neuausrichtung des Gesamtsystems gesteuerte Lösung vieler eher kleinteiliger Probleme bedeutet. Hierin liegt ein potenziell hoher Koordinationsauf- 
wand begründet sowie die Gefahr, dass die Umsetzung jeder Veränderung nicht intendierte Auswirkungen auf derzeit gut funktionierende Systemelemente haben kann. Die Tatsache, dass die Weiterentwicklung des Status quo keine grundsätzlich neue Aufstellung des Systems bedeutet, kann dennoch den Blick darauf nicht verstellen, dass auch hier bereits eine Einbeziehung und Verpflichtung vor allem des Finanzministeriums erfolgen muss. Generell sollte eine derartige Optimierung zur Vermeidung des erneuten Entstehens von Bypässen und bilateralen Umgehungsversuchen umfassend kommunikativ und mit entsprechenden Handlungsanweisungen begleitet werden.

Option 2: $\quad$ Weiterentwicklung des Status quo

\begin{tabular}{|c|c|}
\hline Governance & Finanzierung \\
\hline $\begin{array}{l}\text { Eigentümerstrategie mit klar formulierten Zielen } \\
\text { und Aufgaben für die Agentur, inkl. output- und } \\
\text { wirkungsorientierten Kennzahlen auf Agentur- } \\
\text { ebene }\end{array}$ & $\begin{array}{l}\text { Wenn Mittel nicht ausgeschöpft werden: } \\
\text { Verbleib der Budgets bei den Agenturen; } \\
\text { Verwendung nach Absprache mit Eigen- } \\
\text { tümer bzw. Auftraggeber }\end{array}$ \\
\hline $\begin{array}{l}\text { Entwicklung des Mehrjahresprogramms auf Ba- } \\
\text { sis der strategischen Intelligenz in enger Koope- } \\
\text { ration zwischen Prinzipal und Agentur }\end{array}$ & \multirow[t]{3}{*}{ Teilkostenrechnung in beiden Agenturer } \\
\hline $\begin{array}{l}\text { Transparente Zuweisung von Programmen an } \\
\text { die Agenturen nach Hauptkriterien (aws: Über- } \\
\text { führung in den Markt; FFG: Industrielle For- } \\
\text { schung und Experimentelle Entwicklung) }\end{array}$ & \\
\hline $\begin{array}{l}\text { Beauftragung von Evaluierungen durch die Ei- } \\
\text { gentümer mit Einbezug der Agenturen, um ge- } \\
\text { meinsames Lernen zu maximieren; ausnahms- } \\
\text { lose Veröffentlichung aller Evaluierungsberichte }\end{array}$ & \\
\hline Aufgaben & $\begin{array}{l}\text { Organisationsstrukturen und } \\
\text { Managementprozesse }\end{array}$ \\
\hline $\begin{array}{l}\text { Entwicklung der Förderinstrumente/-programme } \\
\text { durch die Agenturen, im Auftrag der Ministerien }\end{array}$ & \multirow{3}{*}{$\begin{array}{l}\text { Verpflichtung der Agenturen zur klaren } \\
\text { Kommunikation der Auftraggeber bzw. } \\
\text { Eigentümer und Finanziers gegenüber } \\
\text { Förderinteressenten und -kundInnen }\end{array}$} \\
\hline $\begin{array}{l}\text { Zielgruppenmobilisierung, Zielgruppenansprache } \\
\text { und Beratung durch die Agenturen, auch für alle } \\
\text { missionsorientierten Programme }\end{array}$ & \\
\hline $\begin{array}{l}\text { Gemeinsame Entwicklung strategischer Intelli- } \\
\text { genz bei Prinzipal sowie Agentur }\end{array}$ & \\
\hline
\end{tabular}

Im Zentrum einer Optimierung des Status quo stehen zunächst die Stärkung der strategischen Funktion bei den Eigentümerministerien sowie die gleichzeitige Stärkung der Agenturen durch mehr Autonomie in der Umsetzung. Zentral ist dabei, dass die Möglichkeit einer unterjährigen, zusätzlichen Beauftragung erhalten bleibt und somit die Politik 
in die Lage versetzt wird, auch in einer Situation mit autonomeren Agenturen Schwerpunktsetzungen vorzunehmen oder kurzfristig notwendige Maßnahmen zu implementieren. Daher bliebe auf dem Weg vor allem in ihrer Eigenschaft als Eigentümer die Durchgriffsmöglichkeit der Ressorts auf die Agenturen intakt.

Zusätzlich zur allgemeinen Stärkung der Eigentümerfunktion im Vergleich zur Auftraggeberfunktion würden die Ressorts in die Lage versetzt, mittels KPIs nicht mehr nur die Agenturaufgaben, sondern auch die Wirkungsdimensionen des Agenturhandelns zu überprüfen; die Governance müsste hinsichtlich der entsprechenden Einstellungen auf eine Wirkungsorientierung umgestellt werden. Die Zahl der KPIs ist dabei möglichst gering zu halten. Dabei ist es allerdings notwendig, die derzeitige Praxis der Ausschreibung von Evaluierungen hinsichtlich Verantwortlichkeit (Ressorts und Agenturen) sowie Veröffentlichung zu vereinheitlichen. Die Legitimation des Interventionshandelns würde insgesamt erhöht und Informationsasymmetrien zwischen den Akteuren deutlich reduziert. Zudem würde die verpflichtende Kommunikation der Programmeigentümer bzw. Finanziers die Sichtbarkeit der Ressorts gegenüber den Fördernehmern und -interessenten verbessert (z.B. durch Aufnahme in den Rahmenvertrag).

Die Verzögerung von Programmstarts bzw. -weiterführungen könnte in einem solchen Szenario mittels Reduktion von Ausführungsverträgen vermindert werden. Zudem blieben nicht verbrauchte Budgets für das ursprünglich vorgesehene Thema erhalten oder könnten bewusst, d.h. im Sinne einer aktiven Steuerung in andere Themen mit höherer Dynamik gelenkt werden. Dazu wären entsprechende Austausch- und Diskussionsprozesse sowie Entscheidungsstrukturen zu entwickeln.

Im Verhältnis der Ressorts zu den Agenturen käme es durch eine Teilkostenrechnung in beiden Agenturen zu einer Erhöhung der Kostentransparenz, was wiederum die Gleichbehandlung der Agenturen bei Programmbeauftragungen ermöglicht. Schließlich würden die Agenturen deutlich größere Freiheiten im Design sowie in der Umsetzung von Instrumenten/Programmen erhalten, bei gleichzeitiger Stärkung der Kontrollfunktion seitens der Ressorts. Durch die höhere Entscheidungskompetenz in den Agenturen ließen sich insbesondere Zielgruppenansprache und Kundenorientierung verbessern.

Auch hinsichtlich der vier Ziele der Strukturreform würde die Umsetzung dieser Zukunftsoption eine weitere Reduktion der organisatorischen Komplexität mit sich bringen, da die unterschiedlichen Rahmenbedingungen einzelner Agenturbereiche weiter harmonisiert würden. Die verbesserte Zielgruppenansprache würde zudem auch die Steuerungsfähigkeit gegenüber den Zielgruppen verbessern. 


\subsubsection{Agencification Stufe 3: Höhere Autonomie durch Globalsteuerung}

Diese Zukunftsoption manifestiert den Übergang von der Detailsteuerung hin zu einer ergebnisorientierten Steuerung "auf Abstand", die mittels eines Globalbudgets zwar die gewünschten Wirkungen der Agenturen mittels Zielformulierung bzw. thematischen Schwerpunkten und der Definition von Wirkungsindikatoren vorgibt, die konkrete Verteilung der Budgets auf Instrumente jedoch großteils den Agenturen überlässt. Damit erfolgt eine Übertragung von mehr Entscheidungskompetenz und Verantwortung an die Agentur, während sich der Prinzipal aufgrund der entstehenden Freiräume verstärkt auf die politisch-strategischen Aspekte, die Gestaltung von strukturierten Lernprozessen zur Minimierung von Informationsasymmetrien (durch Transparenz und Partizipation zur Unterstützung des Agenda-Settings) sowie die Kontrollfunktion fokussiert. Internationale Good-Practice-Beispiele wie die in Kapitel 8 untersuchten VINNOVA, KfW und Finnvera, sind gewichtige Belege für den möglichen Erfolg, der mit einer solchen Ausgestaltung des Prinzipal-Agenten-Verhältnisses erzielt werden kann.

Eine derartige, vollständige Delegation der Umsetzungsaufgaben in die Agenturen muss mit einer entflochtenen, koordinierten Governance einhergehen. Der zentrale Vorteil diese Zukunftsoption besteht darin, dass die Agenturen ihr akkumuliertes Wissen darüber, was funktioniert, effizienter einsetzen und eine entsprechende Kohärenz des Portfolios mit kritischen Größen herstellen können. Die Ressorts andererseits werden hinsichtlich ihrer Kapazitäten und der verfügbaren Instrumente der Steuerung deutlich aufgewertet und gleichzeitig in Bezug auf die ressourcenverbrauchende Detailprüfung etc. entlastet. Auch hier besteht die größte Herausforderung im Fehlen entsprechender Routinen und der Frage der Gestaltung des Übergangs. Aufgrund der notwendigen Anpassung der Verwaltungs- und Steuerungskultur ist ein entsprechender, umfassender Einstellungswandel aufseiten der Agenturen und Ressorts vonnöten. Auch die Einsicht, dass ein Loslassen auf operativer Ebene bei den Ressorts insgesamt zu einer Stärkung der Prinzipal-Agent Beziehung beitragen wird, dürfte in den Eigentümerressorts fallweise erhöhte Überzeugungsarbeit benötigen. Als eine weitere Herausforderung ist damit auch der dementsprechend notwendige "lange Atem" aufgrund der mittel- bis langfristigen Umsetzungsperspektive zu sehen. Über das langfristige Commitment hinaus, besteht eine zentrale Herausforderung darin, sicherzustellen, dass alle Akteure bis hin zum Finanzministerium eingebunden sind und sich der Definition und schließlich Erreichung eines gemeinsamen Ziels verpflichten. Dieser Prozess sollte grundsätzlich extern begleitet werden, um zu gewährleisten, dass sich Steuerungsverständnis, Aufgabenzuschreibung und Kommunikationspraxis aller handelnden Personen und Organisationen gemeinsam in die gleiche Richtung entwickeln. 
Option 3: $\quad$ Höhere Autonomie durch Globalsteuerung

\begin{tabular}{l|l}
\hline Governance & Finanzierung \\
\hline $\begin{array}{l}\text { Strategische Steuerung ausschließlich über Ei- } \\
\text { gentümerfunktion (Rahmenvertrag und Mehrjah- } \\
\text { resprogramm, stärker genutzte und gezieltere } \\
\begin{array}{l}\text { Weisungsbefugnis gegenüber Geschäftsführung } \\
\text { der Agenturen) }\end{array}\end{array}$ & $\begin{array}{l}\text { Globalbudget auf Basis eines verbindli- } \\
\text { chen Mehrjahresbudgets (5 Jahre), mit } \\
\text { einem detailliert erarbeiteten jährlichen } \\
\text { Arbeitsprogramm }\end{array}$ \\
\hline Aufgaben & $\begin{array}{l}\text { Organisationsstrukturen und } \\
\text { Managementprozesse }\end{array}$ \\
\hline $\begin{array}{l}\text { Entwicklung und Umsetzung der Förderinstru- } \\
\text { mente/-programme durch die Agenturen }\end{array}$ & $\begin{array}{l}\text { Koordination zwischen Agenturen auf } \\
\text { strategischer und operativer Ebene mit- } \\
\text { tels Rahmenvertrag und Mehrjahrespro- } \\
\text { gramm sowie jährlicher Arbeitspro- } \\
\text { gramme }\end{array}$ \\
$\begin{array}{l}\text { Falls nötig bei missionsorientierten Programmen: } \\
\text { matisches Monitoring durch die Agenturen }\end{array}$ & \\
\hline
\end{tabular}

Die Umstellung der Ressort-Agentur-Beziehungen auf Globalsteuerung und -budget zieht eine Umstellung der Interventionslogik auf Themensteuerung und -management nach sich, inklusive einer entsprechenden Evaluierungspraxis, welche die Entwicklung von Themen bzw. die Lösung von gesellschaftlichen Herausforderungen statt Programme evaluiert. Die derzeitigen Eigentümerstrategien müssten grundlegend und dahingehend diskutiert und neu erstellt werden. Damit unmittelbar verbunden ist die vollständige Delegation der Umsetzung an die Agenturen, d.h. ein umfassender Verzicht auf Verfahrenskontrollen sowie Einzelanweisungen und -eingriffe seitens der Ressorts. Dies wird nur unter veränderten Vorzeichen, d.h. in einer vertrauensbasierten Zusammenarbeit unter den strategischen Vorgaben der Politik möglich sein. Gleichzeitig kann sich nur so ein einheitliches Steuerungsverständnis entwickeln.

Im Vergleich zum verbesserten Status quo würde der Administrationsaufwand aller Akteure in dieser Option 3 noch einmal deutlich reduziert. Die Abstimmung der Eigentümer untereinander und mit den Agenturen während der Erstellung der umfassenden Rahmenverträge und der Mehrjahresprogramme würde zwar aufwändiger (letzteres würde auch eine entsprechende Adaptierung der gesetzlichen Grundlagen erfordern), stünde aber in einem proportionalen Verhältnis zu ihrem Nutzen als Instrument der Governance. Die Ressorts würden die Erarbeitung der Mehrjahresprogramme koordinieren und die Vorschläge aus der Wirtschaft (z.B. relevanten Technologie-Roadmaps), von den Agenturen selbst und anderen Stakeholdern zu einem handlungsleitenden Instrument entwickeln. Die große Anzahl von Programmen würde dadurch reduziert bzw. durch Instrumente und Themensetzung ersetzt. 
Die Sichtbarkeit politischen Handelns müsste dann umso mehr als Element der Mehrjahresprogramme durch die Kommunikation der Agenturen mit ihren Fördernehmern sowie in der Erarbeitung (und Veröffentlichung) strategischer Intelligenz ausgedrückt werden. Letztere wäre dann stärker in den Ressorts verankert und würde sie somit als Gegenüber der Agenturen zu stärkeren Partnern machen sowie eine informierte Steuerung ermöglichen.

Die Umsetzung dieser dritten Option würde schließlich einen entscheidenden Beitrag zur Erreichung der formulierten vier Ziele der Strukturreform leisten. Während die Reduzierung inhaltlicher Komplexität aufgrund der Rahmenbedingungen (Regelungen der Europäischen Kommission, wirkungsorientierte Haushaltsführung, generelle Ausdifferenzierung der Wirtschafts- und Innovationspolitik etc.) kaum zu erreichen ist, würde die beschriebene Umstellung des Systems zumindest die Programmvielfalt deutlich reduzieren, zu Gunsten eines verbesserten Themenmanagements. Darüber hinaus wären in dieser Option die wesentlichen Grundlagen geschaffen, um die Reduktion der organisatorischen Komplexität weiter zu treiben. Diese bezieht sich auf die unterhalb der Betrachtungsebene der Agenturen als Ganzes fortbestehenden unterschiedlichen Rahmenbedingungen, was beinahe einer de facto Weiterexistenz der Vorgängerorganisationen gleichkommt. Mit der Umstellung auf eine vollständige Delegation der Umsetzung von Maßnahmen an die Agenturen würden diese Unterschiede so stark reduziert, dass eine echte Integration der verschiedenen Bereiche erleichtert würde. Man denke nur, als ein Beispiel, an die unterschiedliche Ausgestaltung von Programmen, der Prozesse und Antragsformulare des Basisprogramms und der Thematischen Programme in der FFG. Die Zielgruppensteuerung ließe sich durch flexiblere und integrierte Strukturen und kurze Lernschleifen noch weiter verbessern. Darüber hinaus wäre die Erreichung von Regierungszielen im Sinne der Effizienz durch die vollständige Delegation und klare Arbeitsteilung deutlich verbessert. 


\section{Fazit und Handlungsempfehlungen}

\section{$10.1 \quad$ Fazit}

Als Fazit der Evaluierung lässt sich festhalten, dass beide Agenturen nach einem erfolgreich vollzogenen Fusionsprozess hochprofessionell agieren. Im Zuge der Fusion und aufgrund der Zusammenführung nicht-homogener Vorläuferorganisationen sowie der dadurch entstandenen relativ großen Organisationseinheiten wurden nicht zuletzt zur Stärkung der Effektivität und Effizienz der delegierten Aufgaben (d.h. in erster Linie Umsetzung der entsprechenden Förderaktivitäten vor allem der beiden Eigentümerressorts bmvit und bmwfw) zahlreiche Prozesse und Strukturen implementiert, die sich im Großen und Ganzen bewährt haben. Um die Zielerreichung näher auszuleuchten, wird in Folge auf die vier Strukturreformziele für die Gründung der beiden Agenturen einzeln eingegangen.

Gemäß Austria Wirtschaftsservice-Errichtungsgesetz sowie dem Österreichischen Forschungsförderungsgesellschaft $\mathrm{mbH}$-Errichtungsgesetz lagen der Strukturreform im Förderungsbereich mit der Gründung der FFG und aws die folgenden Ziele und Annahmen zugrunde:

- Reduktion organisatorischer und inhaltlicher Komplexität;

- Lösung immanenter Koordinationsprobleme durch die Schaffung von ressortübergreifenden Förderungsgesellschaften;

- Erhöhung der politischen Steuerungsfähigkeit gegenüber den Zielgruppen;

- Verbesserung der Umsetzungschancen von Regierungszielen, nämlich Effizienz und Intensivierung des Ressourceneinsatzes.

Diese Ziele sind vor dem Hintergrund zu sehen, dass die beiden Organisationen aus dem Zusammenschluss von jeweils vier Vorgängerorganisationen hervorgingen, durch ihre Entstehung das gesamte Fördersystem jedoch über die mit einer solchen Fusion einhergehenden Herausforderungen hinaus professionalisiert und optimiert werden sollte. Insbesondere sollte eine Modernisierung des gesamten politischen und verwaltungsbezogenen Handelns in den zentralen Politikfeldern erreicht werden, auch wenn die 2004 definierten Ziele dies nur implizit benennen.

Die Fusion der Vorläuferorganisationen als Gesamtprozess hat zu einer weitgehenden Homogenisierung innerhalb der neu geschaffenen Agenturen insbesondere mit Bezug zu den dort jeweils entwickelten und angewandten Prozessen geführt. Allerdings existieren nach wie vor deutliche Unterschiede hinsichtlich der jeweils geltenden Rahmenbedingungen wie etwa die unterschiedliche Finanzierungsbasis der einzelnen Bereiche, die Aufgabenprofile (auch und insbesondere die Arbeitsteilung mit den Eigentümern 
bzw. Auftraggebern), sowie die unterschiedlichen Beauftragungsverhältnisse durch die Auftraggeber. Zum Teil bestehen die Vorläuferorganisationen nunmehr als Abteilungen weiter und haben bedingt durch die genannten Rahmenbedingungen mit sehr heterogenen Zugängen und unterschiedlichen Herausforderungen zu kämpfen. Nicht zuletzt durch den bislang wenig ausgeprägten konzertierten Zugang der Eigentümer zu den Agenturen, dem fehlenden einheitlichen Steuerungsverständnis sowie dem aus der nicht vollständigen Delegation der Umsetzungsaufgaben an die Agenturen resultierenden System einer Vielzahl von Einzelbeauftragungen ist eine weitgehende Reduktion organisatorischer Komplexität nicht vollumfänglich erreicht worden. Hier gibt es dementsprechend noch Entwicklungspotenzial zur weiteren Homogenisierung und Vereinfachung. Diese Möglichkeiten können jedoch nur ausgeschöpft werden, wenn die Eigentümer die Rahmenbedingungen ebenfalls weiterentwickeln und weitgehend harmonisieren.

Eine Reduktion der inhaltlichen Komplexität wurde mit der Umsetzung der Strukturreform und auch in weiterer Folge nicht erreicht. Dies ist einerseits durch die zunehmende inhaltliche Komplexität der Rahmenbedingungen (Regelungen der Europäischen Kommission, wirkungsorientierte Haushaltsführung, generelle Ausdifferenzierung der Wirtschafts- und Innovationspolitik und Erweiterung um neue Technologie- und Handlungsfelder etc.) begründet, die nicht nur für zusätzlichen Aufwand bei den Ressorts sowie Agenturen sorgte, sondern auch bei den KundInnen im Zuge der Beantragung und Abrechnung von Projekten. Darüber hinaus hat sich die Anzahl an beauftragten Initiativen beständig erhöht, d.h. der bereits in der Systemevaluierung beschriebene "Förderdschungel" bzw. für die Förderprofis eher der "Fördersupermarkt" (Mayer et al. 2009) existiert nach wie vor. Auch aufgrund einer stärker an Sichtbarkeit interessierten Politik, die vor allem durch zusätzliche Förderangebote hergestellt werden soll, besteht somit eine große Anzahl an Programmen, wovon manche unterkritische Größen aufweisen22 (vgl. RH-Bericht zur Forschungsfinanzierung 2016) oder aufgrund der Interventionslogik ein Zusammenlegen mit anderen Programmen überlegenswert scheint. Selbst vor dem Hintergrund einer gestiegenen Komplexität von Handlungsfeldern und Bedarfslagen sowie der Einschätzung, dass der überwiegende Teil der Programme als maßgeschneiderte Antwort darauf zu sehen ist, ist die Gesamtkomplexität des Förderangebots deutlich gestiegen und macht es für diejenigen Fördernehmer schwierig, Zugang zu finden, die bislang über wenig Fördererfahrung verfügen.

Die Lösung immanenter Koordinationsprobleme durch die Schaffung von ressortübergreifenden Förderungsgesellschaften spricht mehrere Ebenen der Koordination an:

22 Es gibt keine exakte Definition dessen, ab wann ein Programm als unterkritisch anzusehen ist, da dies je nach Kontext stark variiert. Ansatzpunkte sind jedoch die Laufzeit, die Anzahl der Förderfälle bezogen auf die Zielgruppe sowie das Budget für die Maßnahme. 
(1) zwischen den Ministerien und den Agenturen im Rahmen ihrer Eigentümer- und Auftraggeberfunktion, (2) zwischen den Agenturen und den Bundesländern, und (3) zwischen den Agenturen und den Ministerien hinsichtlich der Unterstützung der Agenden der Ministerien mit der Europäischen Kommission und andere internationale Initiativen. Während die Koordination von Beziehungen der Punkte 2 und 3 durchaus im Vergleich zurzeit vor den Fusionen als zufriedenstellend gewertet werden können, auch wenn die Abstimmung mit den Bundesländern nach wie vor als kompliziert angesehen wird, ist die Zielerreichung bei der Lösung von Koordinationsproblemen mit den Eigentümerministerien nur als bedingt erreicht einzustufen. Die noch verbleibenden Koordinationsprobleme beruhen auf einer unklaren Aufgabenteilung zwischen Agenturen und Ressorts, die zu einer Untersteuerung auf strategischer Ebene und einer teilweisen Übersteuerung auf operativer Ebene führt. Dies ist ein Befund, der bereits in der Systemevaluierung des österreichischen FTI-Systems im Jahr 2009 erhoben wurde. In der Zwischenzeit wurden zwar von der Politik bedeutende Anstrengungen zur Koordination und Steuerung der FTIPolitik insgesamt vorgenommen (ressortübergreifende FTI-Strategien, höhere Budgets etc.), auf Agenturebene wurden die gesetzlichen Grundlagen reformiert (KMUFörderungs- und Garantiegesetz, FTFG, FTI und FFG Richtlinien) sowie die Rahmenverträge überarbeitet, es erfolgten jedoch kaum Änderungen hinsichtlich der Governance der beiden Agenturen. Letzteres spricht einerseits die Tatsache an, dass die Agenden/Eigentümerinteressen der FFG sowie der aws nach wie vor auf zwei Ministerien verteilt sind und somit eigentlich einer intensive(re)n Koordination bedürfen, und andererseits eine strikt(er)e Trennung zwischen den strategischen sowie ausführenden Rollen erforderlich wäre, wobei sich die Ressorts auf die strategische Funktion konzentrieren und den Agenturen die Implementation überlassen sollten.

Die Erhöhung der politischen Steuerungsfähigkeit gegenüber den Zielgruppen ist im Vergleich zu Situation vor der Strukturreform als positiv zu bewerten. Durch die Schaffung der beiden Agenturen hat sich die Förderlandschaft vereinfacht, und es lassen sich einzelne Zielgruppen leichter in einer abgestimmten Art und Weise erreichen. Dies zeigt sich beispielsweise am Thema FuE-intensive Start-up Förderung. Das derzeit angebotene Portfolio wäre vor der Strukturreform auf vier bis fünf Organisationen aufgeteilt, mit allen einhergehenden Such- und Transaktionskosten sowie Komplikationen in der Administration für die Zielgruppe. Ein weiteres Beispiel stellt die Kombination von ERPKrediten mit unterschiedlichen Garantieleistungen dar, die von den Unternehmen auch stark nachgefragt werden.

Eine weitere Herausforderung stellt das missionsorientierte Förderportfolio bei sehr neuen bzw. dynamischen Themenfeldern dar, das teilweise einer besonderen Behandlung bedarf (z.B. in den Bereichen Energie und Mobilität), da die Entwicklung von capa- 
bilities/Kompetenzen, das community building, die Vernetzung sowie der Wissenstransfer bei missionsorientierten Programmen eine noch bedeutendere Rolle einnimmt und damit noch aktiver von den Agenturen unterstützt werden sollte.

Die Verbesserung der Umsetzungschancen von Regierungszielen, nämlich Effizienz und Intensivierung des Ressourceneinsatzes (Effektivität), stellt die Funktionalität der Umsetzungsebene in den Mittelpunkt der Betrachtung. Hier ist festzustellen, dass die beiden Agenturen, gegeben den ihnen gesetzten Rahmenbedingungen, durchaus erfolgreich agiert haben. Bezüglich der Effizienz des Handelns wurde die Abwicklung der Förder- und Finanzierungsportfolios über die Jahre deutlich modernisiert und die Zielgruppen sind mit der Kommunikations- und Dienstleistung der Agenturen grosso modo sehr zufrieden. Die Abwicklungskosten haben sich bei der FFG im Beobachtungszeitraum (2006-2016) in Relation zu den Fördermitteln kontinuierlich reduziert, trotz gestiegener Aufgabenvielfalt. Für die aws lässt sich festhalten, dass die Implementierung einer Vielzahl moderner Management-Instrumente zu insgesamt optimierten und damit effizienteren Prozessen geführt hat. Dies zeigt sich nicht nur an einer Halbierung des timeto-contract, sondern auch anhand von deutlichen Produktivitätssteigerungen.

Um der Frage nachzugehen, wie sich die Effektivität der Umsetzung der Strukturreformziele in Zukunft noch weiter erhöhen lässt, wurden im Rahmen der Evaluierung drei Zukunftsoptionen entwickelt: die Weiterführung des Status quo, die Weiterentwicklung des Status quo und schließlich die vollständige Agencification mit voller Agenturautonomie und unter Einsatz von Instrumenten einer Globalsteuerung.

Die Agenturen sollten dahingehend entwickelt werden, dass sie dynamisch, risikobereit und verantwortlich Förderportfolios selbst gestalten und politisch gesetzte Prioritäten adressieren und umsetzen können. Die entsprechenden Rahmenbedingungen müssen gleichzeitig die Steuerungsfähigkeit der Eigentümerressorts stärken (nicht zuletzt, um der Problematik ansonsten fehlender mittelbarer demokratischer Kontrolle und Legitimierung zu begegnen und die Agenturen als Instrument der Umsetzung politischer Willensbildung zu erhalten) und das Governancesystem auf eine vertrauensbasierte, ergebnisorientierte Grundlage stellen. Dies lässt sich sowohl aus dem Literaturkreis zum Thema Governance aus anderen Politikfeldern als auch den konkreten Fallbeispielen in dieser Evaluierung ableiten.

Insgesamt sieht das Evaluationsteam eine klarere operationale (auf die Umsetzung wirtschafts- und innovationspolitischer Impulse und Ideen bezogen) und finanzielle Autonomie vor allem durch eine weitergehende Delegation an die Agenturen als erstrebenswert an, nicht zuletzt auch deshalb, weil dieser Weg durch internationale Beispiele bereits erfolgreich umgesetzt wurde. 


\subsection{Handlungsempfehlungen}

Auf Basis der oben zusammengefassten wesentlichen Erkenntnisse der vorliegenden Evaluierung wurden neben der Entwicklung von Zukunftsoptionen weitere, teilweise in den Optionen enthaltene Handlungsempfehlungen erarbeitet. Die Umsetzung dieser Empfehlungen erscheint uns mit Blick auf die Stärkung der Leistungsfähigkeit des Systems auch unabhängig von einer Entscheidung für eine der drei dargestellten Optionen zentral.

Den folgenden Handlungsempfehlungen muss vorangestellt werden, dass die oben zusammengefassten kritischen Befunde zwar den entsprechenden Handlungsraum abstecken, das System in vielen Teilen jedoch durchwegs gut funktioniert. Es gibt einige Evidenz dafür, dass in bestimmten Bereichen die Rahmenbedingungen aufgrund ihrer individuellen Ausgestaltung und Interpretation eine bessere Governance und optimierte Leistungsfähigkeit ermöglichen. Dort finden sich dann auch eine eher vertrauensbasierte Arbeitsteilung sowie die oft zitierte Konzentration beider Seiten (Ressorts und Agenturen) auf ihre jeweiligen Kernaufgaben. Dennoch lassen sich aus den Befunden folgende Optimierungspotenziale ableiten:

- Die vielen ressourcenbindenden Ausführungsverträge sollten schnellstmöglich reduziert werden, um es den Agenturen und Ressorts zu ermöglichen, sich vermehrt auf ihre Kernaufgaben zu fokussieren. Zum Beispiel laufen in der FFG über 70 Ausführungsverträge parallel, da diese nicht nur für einzelne Programme, sondern teilweise sogar pro Call abgeschlossen werden. Um dies zu vermeiden sollte die Steuerung der Agenturen selbst stärker als bisher über die Eigentümerfunktion erfolgen. Dazu sind die vorhandenen Instrumente und insbesondere die Rahmenverträge mit den Agenturen so zu gestalten, dass einerseits die Anzahl der notwendigen Ausführungsverträge deutlich reduziert wird und andererseits die Arbeitsteilung deutlich genug geregelt wird, um handlungsleitend wirken zu können. Dabei sollten die bestehenden Gestaltungsmöglichkeiten hinsichtlich längerer finanzieller Planungshorizonte aktiv genutzt werden. Insbesondere die entstehenden Verzögerungen bei Ausschreibungen sowie in der Bereitstellung bewilligter Mittel stellen dabei ein prioritäres Handlungsfeld dar.

- In diesem Zusammenhang ist auch die teilweise unverhältnismäßig komplexe Situation der Mittelherkunft (Ressorts, Nationalstiftung, Österreichfonds, Garantien bei aws, etc.) anzusprechen. Diese erhöht die Zahl und Komplexität der Programme und Prozesse sowie ihre Kleinteiligkeit und Planungsunsicherheit. Derzeit weisen einige Programme bis zu drei unterschiedliche Finanzierungsquellen auf. Die Finanzierung von Programmen sollte im Sinne eines strategischen Themenmanagements (Effektivität) sowie der Optimierung der Verwaltungseffizienz einheitlich gesteuert werden. Bestenfalls durch ein Globalbudget für die Agenturen und thematische Vorgaben wie in der oben skizzierten Option 3 beschrieben. 
- Darüber hinaus sollten die Eigentümer in einen begleiteten Diskurs zur Erarbeitung eines einheitlichen oder zumindest abgestimmten Steuerungsverständnisses eintreten. Darauf aufbauend wäre die Delegation der Umsetzungsaufgaben an die Agenturen und ihre damit verbundene operationale Autonomie zu klären, um eine entsprechende Standardisierung der tatsächlich gelebten Arbeitsteilung zwischen Auftraggebern und Agenturen zu ermöglichen.

- Im Sinne einer weiteren Reduktion der bestehenden (und zukünftigen) Überschneidungen zwischen den Portfolios beider Agenturen wird empfohlen, explizite Kriterien für die Vergabe des Programm-Managements an eine der beiden Agenturen zu entwickeln. Damit ließen sich die Überlappungsbereiche noch weiter reduzieren, jedoch nicht völlig beseitigen. Da eine trennscharfe Abgrenzung nicht gänzlich möglich ist, muss eine gute Zusammenarbeit und Abstimmung an diesen Randbereichen erfolgen, die bereits im Stadium des Designs der Intervention beginnt. Auch unter der Perspektive einer größeren operativen Unabhängigkeit der Agenturen kann eine Kooperation der Agenturen erwirkt werden, wenn klar kommuniziert wird, welchen Kriterien ein vorgeschlagener Instrumentenmix zu entsprechen hat. Dies bedeutet in der Praxis, dass ein Vorschlag der Agenturen nur akzeptiert wird, wenn eine Abstimmung im Vorfeld erfolgte, und im berechtigten Fall ein gemeinsames Paket angeboten wird.

- Die Ressorts sollten im Rahmen ihrer Eigentümerfunktion eine größere Sichtbarkeit gegenüber den Fördernehmern erwirken, etwa über entsprechende Kommunikationsregelungen im Rahmenvertrag zwischen Agentur und den Ressorts.

- Nicht zuletzt zur Stärkung der Wirkungsorientierung und der Möglichkeit des entsprechenden Nachweises sowie der damit steigenden strategischen Intelligenz in den Ressorts wird empfohlen, entsprechende Sekundärdatenbasen in Österreich zusammenzuführen und EvaluatorInnen zugänglich zu machen. Die Ressorts könnten diese Basis dann nutzen, um noch bessere Evidenz zur Entscheidungsunterstützung über die Weiterführung von Portfolios etc. erarbeiten und steuernd einsetzen zu können. Insbesondere eine Zusammenführung von Förderdaten der Statistik Austria, des BMF sowie der Agenturen und der Bundesländer wäre von hohem Wert. 


\section{Literatur und Quellen}

Aiginger, K./Falk, R./Reinstaller, A. (2009): Reaching Out to the Future Needs Radical Change. Towards a New Policy for Innovation, Science and Technology in Austria. Synthesis Report. Wien: Österreichisches Institut für Wirtschaftsforschung (Wifo).

Arnold, E. (ed.) (2004): Evaluation of the Austrian Industrial Research Promotion Fund (FFF) and the Austrian Science Fund (FWF). Synthesis Report. Zürich: Eidgenössische Technische Hochschule.

Arnold E./Boekholt, P. (2003): Research and Innovation Governance in Eight Countries. A Meta-Analysis of Work Funded by EZ (Netherlands) and RCN (Norway). Brighton: Technopolis.

Aws Mehrjahresprogramme 2014-16 sowie 2017-19. Austria Wirtschaftsservice Gesellschaft $\mathrm{mbH}$, Wien.

Bach, T./Fleischer, J./Hustedt, T. (2010): Organisation und Steuerung zentralstaatlicher Behörden: Agenturen im westeuropäischen Vergleich. Berlin: Edition Sigma.

Bericht des Rechungshofs (2015): FFG und FWF - Interne Kontrollsysteme. Bund 2015/7.

bmvit/bmwfw (2015): Teilnahmeunterlage Teil B Leistungsbild im Verhandlungsverfahren mit vorheriger Bekanntmachung bezüglich der Evaluierung der Förderungsgesellschaften Austria Wirtschaftsservice $\mathrm{GmbH}$ (aws) und Forschungsförderungsgesellschaft mbH (FFG) vom Dezember 2015.

bmwfw/bmvit (2014): aws Strategie und Zielvorgaben der Eigentümervertreter bmwfw \& bmvit, Wien, S. 2.

Braun, D./Guston, D. H. (2003): Principal-agent theory and research policy: an introduction, Science and Public Policy, 30, 302-308.

Braun, D./Gilardi, F. (2002): Delegation aus der Sicht der Prinzipal-Agent Theorie, Politische Vierteljahresschrift, 43, 147-161.

Braun, D. (1998): The Role of Funding Agencies in the Cognitive Development of Science, Research Policy, 27, 807-821.

Braun, D. (1993): Who Governs Intermediary Agencies? Principal-Agent Relations in Research Policy-Making, Journal of Public Policy, 13, 135-162.

Dinges, M./Kaufmann, P./Biegelbauer, P./Sheikh, S. (2016): Indikatoren zur Erfassung der Wirkungen von Förderungen der FFG. Wien: Österreichische Forschungsförderungsgesellschaft (FFG). 
Downs, A. (1967): Inside Bureaucracy. Boston: RAND Corporation.

FFG (2015): Next Steps To Innovation Leader. Das FFG-Arbeitsprogramm 2016. Wien, September 2015.

FFG (2014): Next Steps To Innovation Leader. Das FFG-Mehrjahresprogramm 20152017. Wien, September 2014.

Glennie, A./Bound, K. (2016): How innovation agencies work: International lessons to inspire and inform national strategies. London: Nesta.

Guston, D.H. (1996): Principal-Agent Theory and the Structure of Science Policy, Science in Public Policy, 23, 229-240.

Hammerschmied, G./Egger-Peitler, I./Höllerer, M. (2008): Evaluierung des Instruments der Flexibilisierungsklausel. Wien: Institut für Public Management, WU Wien.

Heinonen, J./ Smallridge, D./Laaksonen, E./ Stenholm, P./ Claes, W. (2012): Evaluation of Finnvera Plc. Final Report. Publications of the Ministry of Employment and the Economy. Innovation 28/2012.

IFES (2016): Präsentation der FFG Mitarbeiter/innenbefragung 2016.

Lane, J.-E. (2013): The Principal-Agent Approach to Politics: Policy Implementation and Public Policy-Making, Open Journal of Political Science, 3, 85-89.

Mayer, S./Ruhland, S./Fischl, I./Sheikh, S. (2009): Governance in der FTI-Politik im Wechselspiel zwischen Ministerien und Agenturen. Teilbericht zur Systemevaluierung der österreichischen Forschungsförderung und -finanzierung, Wien.

Niskanen, W. A. (1971): Bureaucracy and Representative Government. Chicago.

OECD (2005): Modernising Government. Paris: OECD.

OECD (2015): OECD Reviews of Innovation Policy: Sweden 2016. Preliminary Version, Paris: OECD.

Overmann, S./van Thiel, S. (2016): Agencification and Public Sector Performance: A systematic comparison in 20 countries. Public Management Review, 18, 611-635.

Overmann, S. (2016): Great Expectations of Public Service Delegation: A systematic review. Public Management Review, 18, 1238-1262.

Pichler, R. (2016): Agencification revisited. A Case for the Evaluation of FFG as an Agency. fteval Journal for Research and Technology Policy Evaluation, 41, 37-41 
Pichler, R. (2014): Delegierte Koordination. Zur Koordinationsfunktion intermediärer Organisationen in der österreichischen Forschungsförderung, Österreichische Zeitschrift für Politikwissenschaft (ÖZP), 43, 329-346.

Pichler, R./Stampfer, M./Hofer, R. (2007): Forschung, Geld und Politik. Die staatliche Forschungsförderung in Österreich 1945-2005, Innsbruck.

Pratt, J. W./Zeckhäuser, R. J. (1985): The Structure of Business, Boston.

Rechnungshof (2016): Forschungsfinanzierung in Österreich. Wien.

Schillemans, T./Busuioc, M. (2015): Predicting Public Sector Accountability: From Agency Drift to Forum Drift. Journal of Public Administration Research and Theory 25, 191-215. Doi: 10.1093/jopart/muu024.

Technopolis (2014): In search for a benchmark of impact, effectiveness and efficiency of innovation instruments. Management Summary. Amsterdam.

VINNOVA (2008): VINNOVA's Focus on Impact - A Joint Approach for Logic Assessment, Monitoring, Evaluation and Impact Analysis, VINNOVA Analysis VA 2008:01, ISBN: 978-91-85959-03-7.

VINNOVA (2012): Impacts of Innovation Policy - Lessons from VINNOVA's impact studies. VINNOVA Analysis VA 2012:01, ISBN: 978-91-86517-59-5. 


\section{Abkürzungsverzeichnis}

\begin{tabular}{|c|c|}
\hline ALR & Agentur für Luft- und Raumfahrt \\
\hline ASA & Österreichischen Gesellschaft für Weltraumfragen \\
\hline aws & Austria Wirtschaftsservice \\
\hline BIT & Büros für internationale Forschungs- und Technologiekooperation \\
\hline $\mathrm{BP}$ & Basisprogramme (innerhalb der FFG) \\
\hline BMF & Bundesministerium für Finanzen \\
\hline bmvit & Bundesministerium für Verkehr, Innovation und Technologie \\
\hline bmwfw & Bundesministerium für Wirtschaft, Forschung und Wissenschaft \\
\hline CDL & Christian-Doppler-Labore \\
\hline EFRE & Europäischer Fonds für regionale Entwicklung \\
\hline EGT & Ergebnis der gewöhnlichen Geschäftstätigkeit \\
\hline EIP & Europäische und internationale Programme (innerhalb der FFG) \\
\hline ELER & $\begin{array}{l}\text { Europäischer Landwirtschaftsfonds für die Entwicklung des ländli- } \\
\text { chen Raums }\end{array}$ \\
\hline ERA & European Research Area \\
\hline ERP & European Recovery Program \\
\hline ESA & European Space Agency \\
\hline FEI & Forschung, Entwicklung, Innovation \\
\hline FFF & Forschungsförderungsfonds für die gewerbliche Wirtschaft \\
\hline FFG & Österreichische Forschungsförderungsgesellschaft \\
\hline FGG & Finanzierungsgarantie-Gesellschaft \\
\hline FMA & Finanzmarktaufsicht Österreich \\
\hline FTI & Forschung, Technologie, Innovation \\
\hline FuE & Forschung und Entwicklung \\
\hline FWF & Fonds zur Förderung der wissenschaftlichen Forschung \\
\hline
\end{tabular}




\begin{tabular}{|c|c|}
\hline \multicolumn{2}{|c|}{ Abkürzungsverzeichnis } \\
\hline GF & Geschäftsführung \\
\hline GIN & Global Incubator Network \\
\hline IS & Interne Systeme \\
\hline JTIS & Joint Technology Initiatives \\
\hline KLIEN & Klima- und Energiefonds Österreich \\
\hline KMU & Kleine und mittlere Unternehmen \\
\hline KPC & Kommunalkredit Public Consulting \\
\hline $\mathrm{KPI}$ & Key Performance Indicators \\
\hline NCP & National Contact Point \\
\hline OeNB & Österreichische Nationalbank \\
\hline ÖEB & Österreichische Entwicklungsbank \\
\hline ÖGUT & Österreichische Gesellschaft für Umwelt und Technik \\
\hline ÖHT & Österreichische Hotel- und Tourismusbank \\
\hline ÖKB & Österreichische Kontrollbank \\
\hline OSTA & Office of Science and Technology Austria, Washington D.C. \\
\hline$P \& A$ & Projektcontrolling und Audit \\
\hline SCHIG & Schienen- und Infrastrukturgesellschaft \\
\hline SP & Strukturprogramme (innerhalb der FFG) \\
\hline STS & Stabsstellen \\
\hline TAFTIE & The European Network of Innovation Agencies \\
\hline TP & Thematische Programme (innerhalb der FFG) \\
\hline TRLs & Technology Readiness Levels \\
\hline USP & Unique Selling Point \\
\hline VC & Venture Capital \\
\hline VZÄ & Vollzeitäquivalente \\
\hline
\end{tabular}

